\title{
Measuring Sentence-Level and Aspect-Level (Un)certainty in Science Communications
}

\author{
Jiaxin Pei \\ School of Information \\ University of Michigan \\ pedropei@umich.edu
}

\author{
David Jurgens \\ School of Information \\ University of Michigan \\ jurgenseumich. edu
}

\begin{abstract}
Certainty and uncertainty are fundamental to science communication. Hedges have widely been used as proxies for uncertainty. However, certainty is a complex construct, with authors expressing not only the degree but the type and aspects of uncertainty in order to give the reader a certain impression of what is known. Here, we introduce a new study of certainty that models both the level and the aspects of certainty in scientific findings. Using a new dataset of 2167 annotated scientific findings, we demonstrate that hedges alone account for only a partial explanation of certainty. We show that both the overall certainty and individual aspects can be predicted with pre-trained language models, providing a more complete picture of the author's intended communication. Downstream analyses on $431 \mathrm{~K}$ scientific findings from news and scientific abstracts demonstrate that modeling sentencelevel and aspect-level certainty is meaningful for areas like science communication. Both the model and datasets used in this paper are released at https://blablablab.si. umich.edu/projects/certainty/
\end{abstract}

\section{Introduction}

Expressing certainty about what is known is a necessary characteristic of scientific work as science involves producing knowledge about what was previously unknown (Friedman et al., 1999; Smithson, 2012). Given the natural aversion to uncertainty, existing studies have found that presenting uncertainty in science communications influences people's perception of scientific findings and trust in science (Gustafson and Rice, 2019; Fischhoff, 2012; Van Der Bles et al., 2020). Therefore, understanding how journalists and scientists communicate certainty and uncertainty is critical for understanding the current ecosystem of science journalism and further provides better guidance for uncertainty communication (National Academies of Sciences, Engineering, and Medicine, 2017).

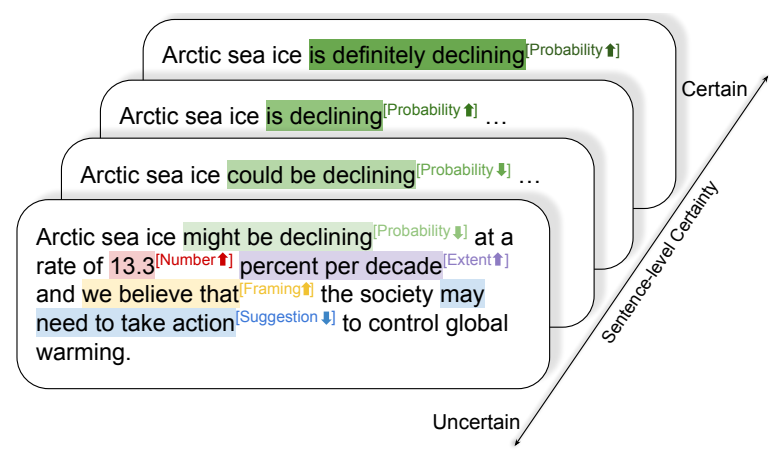

Figure 1: Certainty is a multi-dimensional construct. The certainty of a scientific finding can be perceived holistically at the sentence level from its description. However, scientific findings may involve multiple aspects that may each be described as certain $(\uparrow)$ or uncertain $(\downarrow)$ (aspect-level certainty).

Multiple studies in Linguistics, NLP, and the Science of Science literature have examined how certainty is expressed. These studies have modeled certainty in multiple ways, including epistemic modality (Vold, 2006), semantic uncertainty (Szarvas et al., 2012), verbal uncertainty (Hart and Childers, 2004), factuality (Saurí and Pustejovsky, 2009), and hedging (Hyland, 1996). In practice, most uses of uncertainty rely on hedging as a coarse characterization of the overall uncertainty (Farkas et al., 2010). However, as suggested by Rubin et al. (2006), certainty itself is a complex construct and has to be modeled from multiple dimensions. The complex and subjective nature of certainty makes annotation challenging, often resulting in moderate-to-low annotator agreement (Henriksson and Velupillai, 2010; Rubin, 2007), and motivating a better and more practical way to model and annotate certainty in text.

We propose to study certainty from the commonly-used sentence-level and, in parallel, introduce a new dimension of aspect-level certainty, providing a fine-grained description of how certainty is communicated in text. This approach 
is analogous to work in sentiment analysis that models both holistic (Meena and Prabhakar, 2007), and aspect-level valence (Schouten and Frasincar, 2015) — and their interactions. Based on existing categorizations of certainty, we compile six aspects of scientific findings including: NUMBER, Extent, Probability, Framing, Condition, and Suggestion. Following carefully designed annotation guidelines and after extensive annotator training, we introduce a new annotated corpus of 2200 scientific findings equally, sampled from news and scientific abstracts for both sentencelevel and aspect-level certainty, and attained reliable inter-annotator agreements. Analysis with this dataset suggests that the number of hedges can only partially explain the variance of the overall sentence-level of certainty (Pearson's $r=0.55$ ). Therefore, to better model certainty in scientific findings, we fine-tuned a SciBERT (Beltagy et al., 2019) for the two tasks and it achieves 0.63 Pearson's $r$ for sentence-level certainty and an average of 0.66 binary-F1 for aspect-level certainty.

Our paper offers the following three contributions. First, we provide the first dataset of scientific findings annotated with both sentence-level and aspect-level certainty and fine-tune neural language models to predict certainty in scientific findings. Second, using our best-performing model, we infer the sentence-level and aspect-level certainty for $431 \mathrm{~K}$ scientific findings in news and abstracts and show that the sentence-level certainty of findings in abstracts is associated with journal impact factor and team size. Regression analysis reveals that low-impact journals and large teams often present scientific findings with higher sentencelevel certainty. Third, using 6586 findings from abstracts paired with their description in news, we find that news reports a finding with lower certainty than its corresponding description in the abstract. Fine-grained regression analysis over aspect-level certainty further reveals that journalists describe some key aspects with less certainty. Despite some studies suggest that news reports tend to describe uncertain findings as more certain (e.g., Weiss and Singer, 1988; Fahnestock, 2009), our study indicates that journalists may actually play down the certainty when reporting scientific findings.

\section{Modeling Certainty}

Communicating certainty involves qualifying parts of a statement to indicate what is known and what the speaker's beliefs are. Multiple approaches have proposed models for this property of language. The majority of these studies have focused on the degree or level of certainty (Rubin et al., 2006). Within this approach, hedges have been considered effective proxies for uncertainty (Farkas et al., 2010) across multiple fields including linguistics (Recasens et al., 2013), economics (Ahir et al., 2018), and psychology (Tausczik and Pennebaker, 2010), where the frequent use of hedges indicates more uncertainty and absence indicates certainty. Other work has proposed examining which aspects of language contribute to the perception of uncertainty. For example, Rubin et al. (2006) proposed a four dimension model of certainty, including perspective, focus, timeline, and level, and each dimension contains several sub-categories. Following, we synthesize multiple approaches for measuring sentence-level and aspect-level certainty and proposed a representative categorization.

Sentence-level Certainty Prior research has assumed that the level of certainty for a finding is presented, perceived, and further analyzed within one or several sentences (Holmes, 1982; Henriksson and Velupillai, 2010; Rubin, 2007). This aggregate perception represents a unified perception of various information expressed in the given piece of text and is the primary judgment of certainty along a continuum from uncertain to certain (Rubin et al., 2006). This perception of this overall level of certainty is known to influence people's following actions in many contexts (Corley and Wedeking, 2014; Wood and Eagly, 2009). Therefore, in modeling certainty, we include a sentence-level estimate of the certainty for a scientific finding's description.

Aspect-level Certainty In describing a finding, authors may contextualize which aspects are more or less certain. Many categorizations have been proposed to model the complex nature of these descriptions of certainty and uncertainty including types of uncertainty (Szarvas et al., 2012; Thunnissen, 2003), sources of uncertainty (Politi et al., 2007), issues of uncertainty (Han et al., 2011), forms of uncertainty (French, 1995), focus and perspective of certainty (Rubin, 2007). While prior work focuses on various aspects of certainty, their categorizations are often domain-specific (Politi et al., 2007; Han et al., 2011) or overly limited in scope to cover common aspects appearing in scientific findings (Szarvas et al., 2012; Rubin, 2007). Here, we synthesize prior approaches and propose six repre- 
sentative aspects of scientific findings which could involve certainty or uncertainty, with a goal of creating a comprehensive scheme that captures most of what is seen in knowledge-intensive corpora.

NUMBER refers to certainty towards specific quantities. For example, "approximately 250 individuals participated in this study" is uncertain towards NUMBER. Numerical information is vitally important in science communication as it is found to be the best way to promote scientific understanding in situations like climate change (Budescu et al., 2009) and health (Peters et al., 2014a). Accordingly, the imprecision of numbers or the inaccuracy of calculations are usually considered as a form of uncertainty (French, 1995). How to effectively communicate numerical information in scientific findings has been identified as one of the major challenges of science communication (Peters et al., 2014b). Identifying certainty regarding NUMBER in scientific findings could help to understand how journalists and scientists communicate certainty about numbers and inspire better ways to communicate this information.

EXTENT refers to certainty about the proportion/ratio of properties that make up an object/event or the extent of a change. For example, "This bridge is mainly composed of agate" and "We observe a moderate increase of suicide in Winter" involves uncertainty towards EXTENT. EXTENT can be described with numbers in certain situations. For example, "The average sea level across the world increased by approximately 30\%" expresses extent via a number. However, unlike numbers that focus on specific quantities, EXTENT focuses on the components of an object, substance, or the extent of a change/effect. Previously, EXTENT was not explicitly proposed as a source of uncertainty, although some studies have brought up similar ideas. For example, French (1995) considers "Uncertainty about how much [the] impacts matter" as a form of uncertainty and Phillips et al. (2009) propose uncertainty about the strength or validity of evidence about risks, which may not be described with specific quantities. Existing studies suggest that journalists may misreport the extent of scientific findings to which it is supported by evidence (Dixon and Clarke, 2013), motivating its inclusion here.

PROBABILITY refers to certainty about the probability that something will occur, has occurred, or is associated with another factor. For example, "This medicine could possibly cure cancer" and
"A is possibly associated with B" involves uncertainty about Probability. ProbABILITY has been widely recognized as one major source of uncertainty (Howard, 1988; Mosleh and Bier, 1996; Politi et al., 2007) and how to communicate probabilities effectively has long been an important question in science communication (Budescu et al., 2012; Sinayev et al., 2015).

CONDITION refers to the situation where something depends on a specific condition, and the condition involves certainty or uncertainty (Szarvas et al., 2012). Scientific findings are often qualified by specific conditions under which the result is valid, which may themselves be certain or uncertain (Friedman et al., 1999). For example, "Cancer could be cured if the medicine can be made shelfstable" is uncertain regarding CONDITION.

FRAMING refers to the certainty about how scientists or journalists themselves frame or interpret the scientific finding. For example, "We suspect A has effects on B" involves the uncertainty from the authors while "We conclude that A has effects on B" frames the finding with conviction. This aspect is related to expressions about epistemic uncertainty (the speaker having or lacking knowledge Szarvas et al., 2012; Fox and Ülkümen, 2011) and psychological uncertainty in the Psychology literature (Windschitl and Wells, 1996). In the news, journalists actively add their interpretations about the original information (Lin et al., 2006) and different framing may further affect people's perception of the overall certainty of the presented information (Soni et al., 2014). Therefore, identifying the certainty about FRAMING could help us to better understand how journalist's framing affects people's perceptions of scientific findings.

SUGGESTION refers to certainty or uncertainty about the implications or future actions for the public or science community. Scientific findings do not only describe facts, but can also communicate practical implications for people's daily life (Batteux et al., 2021). For example, "Patients probably need more medicine to cure this disease" involves uncertainty regarding future actions. Uncertainty about SUGGESTION was previously identified as dynamic uncertainty in Szarvas et al. (2012).

A single scientific finding may include multiple aspects with their own certainties. For example, "The vaccine is effective for $76 \%$ of chances" is uncertain regarding PROBABILITY while is certain about the specific Number. Similarly, "The sci- 
entists need to do more research to understand the effect of A on B" indicates the uncertainty about PROBABILITY but is certain about SUGGESTION.

\section{Data}

To study certainty, we construct a dataset of scientific findings reported in news and research articles. News data comes from Altmetrics, which tracks mentions of scientific articles in news outlets. We restrict our analysis to U.S.-based outlets where we could retrieve the full text of the article and where the DOI for the scientific article was recorded in the Microsoft Academic Graph (MAG), which provides metadata on the article (e.g., authors, abstract, and publication venue). Supplemental material §A contains additional details on preprocessing steps. A total of 128,942 news/article pairs were collected, spanning 273 different news outlets and 57,807 different scientific articles.

For scientific articles, we extract the findings from the abstract reported in the MAG using the abstract parser developed by Prabhakaran et al. (2016), which labels sentences as background, method, introduction, result, and conclusion. We use sentences labeled as result or conclusion in our analysis. For news, we adopt a heuristic approach and identify all sentences containing a discoveryrelated keyword (e.g., find, conclude). We retain the subjective clause after the verb as the finding. Examples of findings produced by each method, as well as additional details, are reported in Supplemental Material §C. This process finally leads to 608,694 unique scientific findings from abstracts and 106,612 unique scientific findings from news reports. Among the 128,942 news-paper pairs, 52,406 have both identified findings from news and paper abstracts.

\section{Annotating Certainty}

We annotate scientific findings for both their sentence-level certainty and the presence and certainty of each of the six aspects. Given subjectivity in perception, the same expression of certainty may evoke different perceptions (Druzdzel, 1989). Prior work in annotating uncertainty has generally reported low to moderate inter-annotator agreement (Henriksson and Velupillai, 2010; Rubin, 2007); for example, Rubin (2007) reports Cohen's $\kappa=0.41$ when annotating news certainty with a five-level Likert scale. To mitigate these challenges, we carefully designed the annotation procedures which are described in this section.

Annotation Setup Annotators were recruited from a US university and received initial one-hour training. All annotators are fluent in English and have extensive experience in reading scientific news and research articles. Annotators who attained high IAA with our gold standard were retained and then went through four additional rounds of pilot annotation and discussions ( 2 rounds for sentence-level and 2 for aspect-level) to build consensus. All annotators were paid $\$ 15 / \mathrm{hr}$ for training and annotation.

Annotation was performed in three phases. In the first phase, the initial data was sampled in a way to be more balanced across levels of certainty. Markers of certainty are not equally distributed throughout scientific communication (Rubin et al., 2006); for example, $87 \%$ of the data labeled in Henriksson and Velupillai (2010) were found to be very certain. Therefore, in the initial data, we sample 1000 findings equally from news and paper abstracts where $50 \%$ scientific findings containing no hedges, $35 \%$ with one hedge, and $15 \%$ with $2+$ hedges. The hedge words are collected from Hyland (2005).

The annotators were first asked to rate how certain they perceived the finding on a six-point Likert scale as the sentence-level certainty. Aspect-based ratings were performed in a separate round so as not to potentially bias annotators towards focusing their sentence-level judgments on the basis of aspects. For each aspect, annotators were asked to assess whether that aspect was present and, if so, whether the language for the aspect was certain or uncertain. For instances that are clearly not scientific findings, the annotators were instructed to label it as BAD-TEXT. Each finding was rated by at least two annotators for sentence-level and, due to increased variance observed during training, three annotators for aspect-level. In this phase, 2349 sentence-level and 3209 aspect-level annotations were collected for 1000 findings. Annotators had a high agreement in both sentence-level and aspect-level tasks. For sentence-level, annotators attained a Krippendorff's $\alpha=0.67$, which is substantially higher than IAA for the closest comparable task (Rubin, 2007, Cohen's $\kappa=0.41) .{ }^{1}$ The final sentence-level rating is computed as the average across all annotators' scores. For aspect-level cer-

\footnotetext{
${ }^{1}$ While Cohen's $\kappa$ and Krippendorff's $\alpha$ are not directly comparable, both are chance-corrected scores in $[-1,1]$ and we still view rough comparisons of magnitude useful to provide insight into annotators' relative agreement.
} 

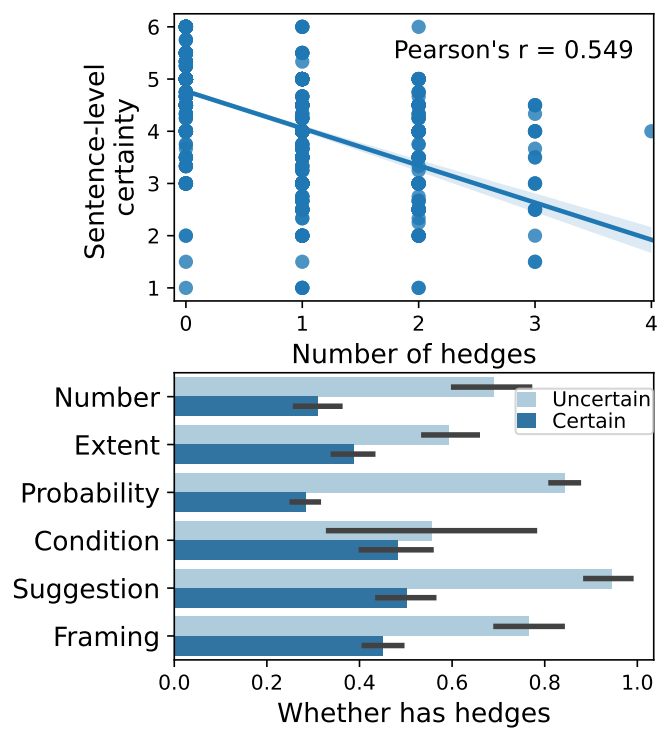

Figure 2: (Top) The number of hedges shows a moderate correlation with sentence-level certainty, while (Bottom) hedges vary in frequency across aspects.

tainty, the average Krippendorff's $\alpha$ is 0.57 for the six aspects, indicating moderate to high agreement. Supplemental material §D contains more details about agreement scores. We take the majority label as the final label for aspect-level certainty.

In the second phase, given the label imbalance in both sentence-level and aspect-level certainty, we sample additional data for annotation based on model predictions using different sampling strategies for sentence-level and aspect-level certainties. For sentence-level certainty, we fine-tuned a roberta-base classification model and predicted all the extracted findings with sentence-level certainty. We further sampled 400 findings with low model confidence equally from news and abstracts for the second-phase annotation. For aspect-level certainty, we fine-tuned a SciBERT classification model and up-sampled 600 findings for less frequent aspects, including SUGGESTION, EXTENT, CONDITION, and FRAMING.

Given that the first and second phase data do not reflect the distribution of certainty in a natural sample, we further randomly sampled 200 findings from all the extracted findings as to the third phase. Given that the annotators were capable of doing reliable annotations after the first phase, annotators independently annotated 1200 findings for the second and third phases.

Results The final annotated dataset contains 6958 labels for 2200 findings. After removing find-

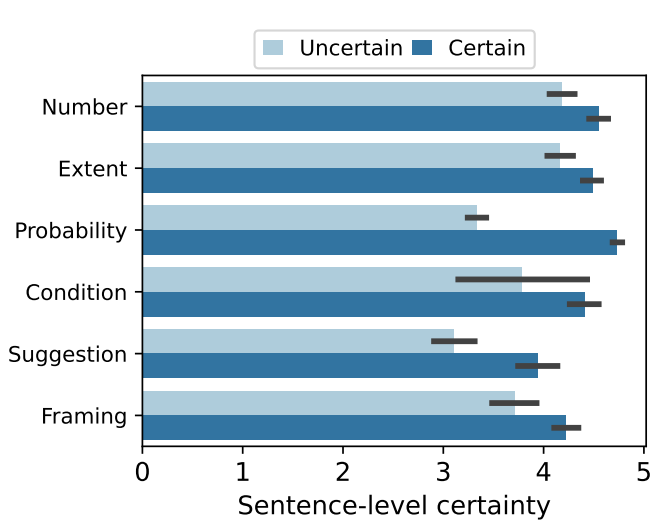

Figure 3: Relative sentence-level certainty when each aspect is certain/uncertain. The overall certainty of scientific findings is majorly affected by PROBABILITY and SugGESTION, while are less affected by other aspects like NUMBER and EXTENT

ings that are labeled as BAD-TEXT, we obtained 1551 findings labeled with sentence-level certainty and 1760 findings labeled with aspect-level certainty, among which 1144 findings are labeled with both sentence-level and aspect-level certainty. Supplemental §E present examples and distribution of the data. Previously, Szarvas et al. (2012) considers CONDITION as one type of uncertainty, however, in the annotated data, less than $10 \%$ of CONDITION is labeled as uncertain. This difference indicates that previously proposed types of uncertainty may not be considered as uncertain in knowledge-intensive corpus like scientific findings and demonstrates the value of aspect-level certainty.

To what degree do hedges capture certainty? Comparing the sentence-level certainty with the number of hedges (Figure 2, top) shows only a moderate correlation between hedging and certainty, $r=0.55$, despite their widespread use as a proxy. For example, "Further research is necessary to understand whether this is a causal relationship" contains zero hedges but explicitly expresses strong uncertainty towards the causal relationship, suggesting that many descriptions of certainty are not well capture by simple hedge-based lexicons. Further, authors vary in how frequently they employ hedges when describing the different aspects of certainty (Figure 2, bottom). This variance in their distribution suggests that hedges are less effective as proxies for capturing uncertainty for all aspects.

What is the association between aspect-level and sentence-level certainty? Figure 3 shows the relative sentence-level certainty for each as- 


\begin{tabular}{rcc} 
Model & $r$ on full test set & $r$ on random set \\
\hline LR + Hedges & -0.02 & -0.02 \\
LR + BoW & 0.55 & 0.44 \\
RoBERTa-base & $0.62 \pm 0.051$ & $0.55 \pm 0.067$ \\
SciBERT & $\mathbf{0 . 6 3} \pm \mathbf{0 . 0 6 1}$ & $\mathbf{0 . 5 7} \pm \mathbf{0 . 0 6 5}$
\end{tabular}

Table 1: Sentence-level certainty performance

pect. Uncertainties about ProbabiLITY and SUGGESTION are associated with a sharp decrease of sentence-level certainty. However, the uncertainties about NUMBER and EXTENT are only associated with a small decrease of sentence-level certainty. This result indicates that the descriptions of aspects vary in how they contribute to the perception of the overall certainty of scientific findings.

\section{Predicting Certainty}

In this section, we build models to predict sentenceand aspect-level certainty in scientific findings to support downstream analyses of certainty. We test two linear baseline models and two deep-learning models based on neural language models. As linear baselines, we include a model using bag-of-words (BoW) features and another based on the frequency of each hedging word. For neural models, we use SciBERT (Beltagy et al., 2019), and RoBERTa model (Liu et al., 2019) as the base models and fine-tune them over our annotated dataset. For both sentence-level and aspect-level certainty, the data labeled in phase 1 and phase 2 are split 8:1:1 into training, validation, and test. To better reflect the expected performance generalization, the test is made from the random set annotated in Phase 3 and the $10 \%$ test partition of Phases 1 and 2. For all the models, we also report their performance on the random test set to demonstrate their performance over the natural samples. Supplemental Section $\S \mathrm{B}$ describes additional training details.

\section{Sentence-level Certainty We formulate} sentence-level certainty prediction as a regression task for all the models and Table 1 shows the model performance. We find that a linear weighting of the hedges is unable to predict the overall sentence-level certainty when tested on the random sample, largely due to the relatively low ratio of findings containing hedges. In comparison, linear regression with bag-of-words features is better able to capture overall certainty with Pearson's $r=0.55$, suggesting that other cues in addition to hedges also affect the overall certainty in the natural

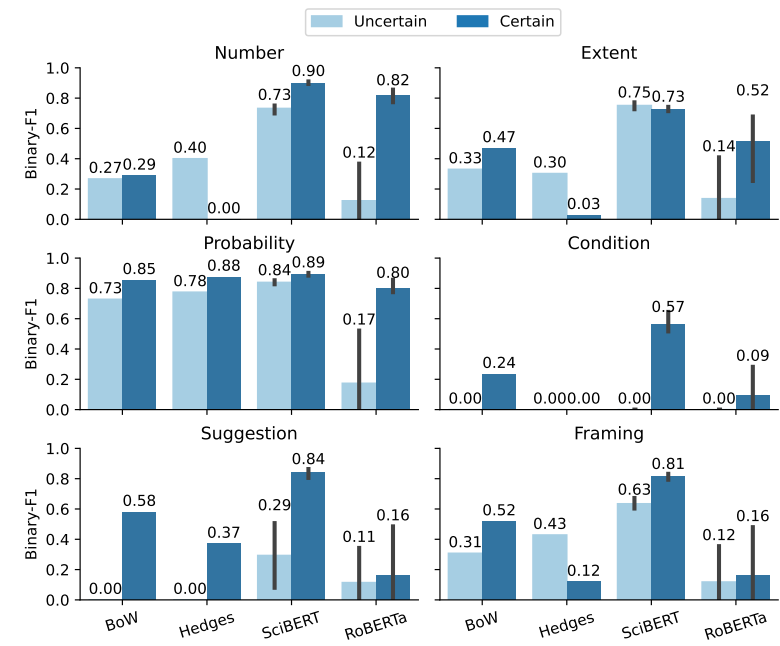

Figure 4: Binary-F1 for predicting aspect-level certainty. SciBERT outperforms all the other baselines.

sample. Compared with the two baselines, the two neural models based on pre-trained language models achieve better performance. Both neural models are run five times with different random seeds, showing that the performance improvements over the baselines are statistically significant $(\mathrm{p}<0.05$ paired $t$-test). The SciBERT model performs slightly better than the RoBERTa-base model, indicating domain-specific pre-training is helpful though not to the point of significance. We use the best-performing SciBERT model $(r=0.70)$ as the regressor for sentence-level certainty in the following analyses.

Aspect-level Certainty For each aspect-level certainty, we predict whether a scientific finding is NotPresent, Certain or Uncertain. For the two neural models, we use shared pre-trained language models but independent classification heads for each aspect. Figure 4 shows the binary-F1 scores for predicting aspect-level certainty. The SciBERT model consistently outperforms other baselines over the six aspects, indicating that aspect-level certainty prediction requires more domain-specific and context information. However, given that uncertainties about CONDITION and SUGGESTION are relatively rare in the annotated dataset, the SciBERT model does not capture well the uncertainty about CONDITION and shows high variance when predicting uncertainty about SUGGESTION. In the following analysis, we use the best-performing SciBERT model (mean F1 $=0.71$ ) as the classifier for aspectlevel certainty. 


\section{Certainty in Science Communications}

Certainty is a core aspect of science communication (Friedman et al., 1999) and presenting certainty in different forms (i.e., aspects) may further affect people's perception and future action about a series of issues including climate change (Fortner et al., 2000) and the Covid-19 vaccine (Batteux et al., 2021). Our models and dataset enable us to study how journalists and scientists present certainty in science communications. Here, we focus on the following five research questions. RQ1: Are findings in science news more certain than those in paper abstracts? RQ2: Do journalists and scientists differ in their use of aspect-level certainty? RQ3: Does aspect-level certainty in abstract findings affect the sentence-level certainty in news findings? RQ4: Does journal impact factor affect the certainty of scientific findings and how they are covered in news reports? RQ5: Does team size affect the certainty of scientific findings and how they are covered in news reports?

RQ1-RQ3 focus on changes to the description of certainty in the science communication process. While studies have found that news reports tend to describe uncertain findings as more certain (Weiss and Singer, 1988; Fahnestock, 2009), some studies suggest that news articles may also add more uncertainty to science finding in some cases (Friedman et al., 1999). Our model and dataset allow us to study (1) if certainty is changed, (2) if so, what aspects are changed, and (3) what drives the change. RQ4-RQ5 examine external factors that may affect how journalists and scientists present certainty. We focus on (4) the prestige/quality of the journal, asking whether lower- or higher-quality journals differ in how certain their findings are, and (5) in the era of team science (Stokols et al., 2008; Hall et al., 2018), whether team size influences how certain the authors describe the results, given known differences in the nature of team science research outputs (Wu et al., 2019; Sud and Thelwall, 2016; Thelwall and Sud, 2016).

Data and method For RQ1 and RQ2, to control the effects of the content of the finding, we propose a method to match the same scientific finding in news and paper abstracts. For each extracted finding in a paper abstract, we identify the paraphrased findings in the corresponding news article reporting on that paper. We first remove all punctuation and stop words and then stem all the words in each sentence. Next, we calculate the overlap and Jaccard similarity between each pair of findings in the news and abstract. We manually evaluated the matched findings and set word overlap $>=3$ and Jaccard similarity $>0.3$ as the threshold. Based on the findings from 52,406 news-paper pairs, we identify 6,586 unique finding pairs from news and abstracts. We manually annotated 70 matched finding pairs, and $63(90 \%)$ refer to the same science finding, indicating high precision of our matching process. Supplemental Material $\S F$ shows a random sample of the matched finding pairs. We construct separate regressions predicting the sentence-level (RQ1) and each aspect-level (RQ2) certainty in findings with the source of the finding (i.e., news or abstract). We further control the fields, author and affiliation ranking, journal impacts, finding length, and Flesch reading ease score. For RQ3, we construct a regression predicting the overall sentence-level certainty in news findings with the aspect-level certainty in the corresponding finding from the paper abstract. Except for all the IVs above, we further control the news outlet and the sentence-level certainty of the finding in the abstract.

For RQ4 and RQ5, we construct a regression predicting the sentence-level certainty in 265,758 findings presented in 55,178 paper abstracts using journal impacts factors and the number of authors. Recognizing its limitations (Kurmis, 2003), we use journal impact factor as a proxy for the quality of science based on prior use (Saha et al., 2003). We include controls the field of the research, author, and affiliation ranking extracted from the Microsoft Academic Graph (Wang et al., 2019), finding length and Flesch reading ease score to remove the potential confounds. To test the connection between certainty in news findings and external factors, we construct another regression to predict the level of uncertainty in 72,013 findings presented in 27,000 news articles. Besides all the IVs regarding abstract and authors, we also control the outlet to remove potential confounds.

RQ1: Are findings in news more certain than those in paper abstracts? The regression analysis (details in Supplemental Table 6) indicates that news descriptions have lower overall sentence-level certainty than abstract descriptions of the same finding $(p<0.01)$. Although existing studies suggest that science news tends to remove hedges and describe science findings with increased certainty (e.g., Weiss and Singer, 1988; Fahnestock, 2009), our study over the paired findings finds the oppo- 


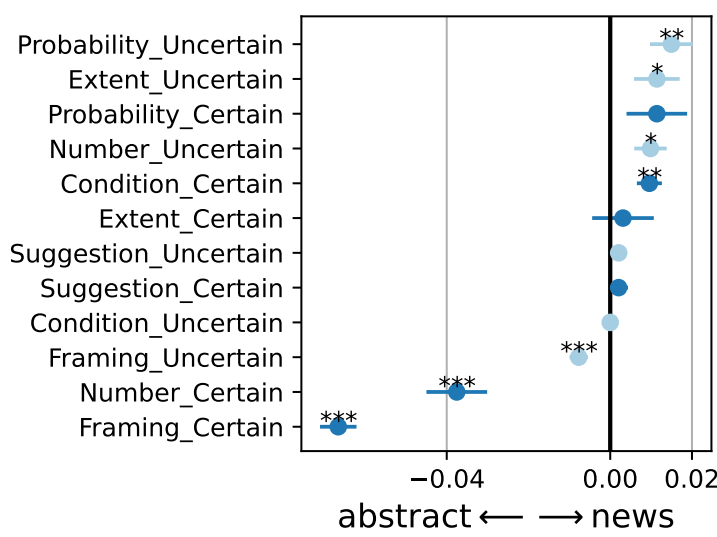

Figure 5: Controlling for multiple factors in RQ2 (e.g., topic, news outlet), the marginal effects show the relative probability of finding each aspect described in the abstract (left) versus news (right), revealing that some aspects like numeric certainty are much more likely to be described in one source.

site: findings in news are less certain compared with findings in abstract, even when controlling the content and many contextual factors.

RQ2: Do journalists and scientists differ in their use of aspect-level certainty? Yes, as shown in Figure 5, findings in abstracts are associated with more certainties about FRAMING and NUMBER. Findings in news are associated with uncertainties about ProbABILITY, EXTENT and NUMBER, indicating that the journalists tend to play down the certainty of some aspects, especially regarding numeric information. Existing studies suggest that laypeople with lower numeracy tend to focus more on narrative instead of numeric information (Dieckmann et al., 2009); one potential explanation for this difference is that journalists could be intentionally simplifying numerical information with hedges like "roughly" instead of the detailed number to better engage the lay audiences. Further, journalists are more likely to discard expressions of the scholar's uncertainty (FRAMING) when presenting the results, potentially aiming to make the work seem more objective. Our result suggests a potential mechanism for lower sentence-level certainty in news compared with abstracts.

RQ3: Does aspect-level certainty in abstract findings affect the sentence-level certainty in news findings? As shown in figure 6, uncertainty about NUMBER and FRAMING are associated with decreased certainty in news findings, indicating that their uncertainty expressions are readily perceived by journalists. However, we also find that the certainty about SUGGESTION in abstracts are

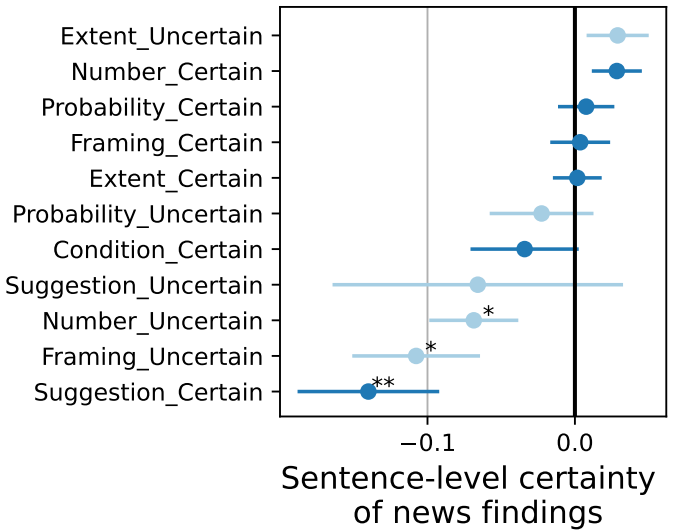

Figure 6: Averaged marginal effects of aspect-level certainty in abstract findings on the level of certainty for matched news findings.

also associated with decreased certainty in news, suggesting that journalists may play down the certainty when presenting the findings involving suggestions or future actions even when it is certain. While existing studies suggest that journalists may exaggerate the potential benefits of science (Wilson et al., 2010), our result indicates that journalists can be very careful when reporting findings involving suggestions or future actions.

RQ4: Are findings in high-impact journals more certain than findings in low-impact journals? No. As shown in Figure 7, findings in the lower-impact journals are written with the highest level of certainty, while findings appearing in relatively higher-impact journals are described with comparatively less certainty. One potential explanation for this phenomenon is that high-quality papers published in journals with more strict reviewing processes $^{2}$ present certainty more precisely, which leads to a lower overall certainty compared with findings in low-impact journals. As a comparison, the certainty of findings written by journalists is not significantly associated with journal impact factors, suggesting that the prestige of a journal does not affect how journalists present scientific findings.

RQ5: Are findings from small teams more certain than findings from large teams? We find a linear relationship between the number of authors and the overall level of certainty in scientific findings (Figure 7), even with controls for fields and authors. Multiple mechanisms may explain this behavior. Larger teams may themselves be more capable of producing more certain results due to

\footnotetext{
${ }^{2}$ Journals with higher impact factors generally have longer reviews than low impact journals (Publons, 2018, p. 36).
} 


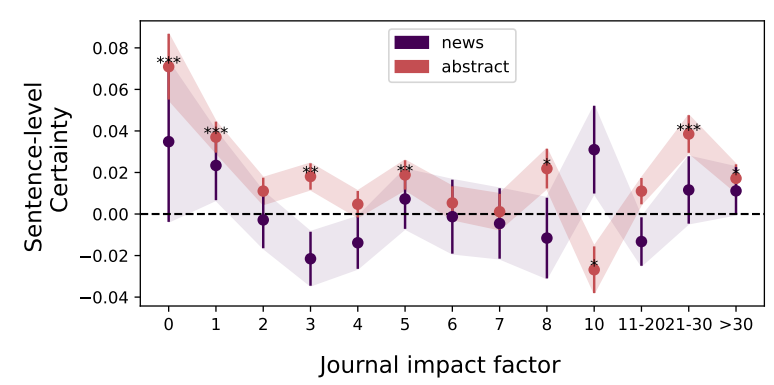

Figure 7: Averaged marginal effects of journal impact factors on level of certainty. Findings appearing on journals with lower impact factors are associated with higher levels of certainty.

more individuals participating and checking results or due to the scale of the experiments capable in team science (Bozeman and Corley, 2004; Jeong and Choi, 2015). Furthermore, our result also connects to Wu et al. (2019)'s finding that small teams generate new disruptive ideas while large teams tend to develop old, existing ideas, as new ideas are often associated with more uncertainties. Alternatively, larger teams likely have more authors who are further away from the experiments and therefore may not be able to describe certainty with the same nuance and precision as smaller teams (or single individuals) who are intimately familiar with the methodological details (MacKenzie, 1998); this distance may increase the perception of certainty when writing. However, this linear trend does not persist in science news; instead, the sentence-level certainty of findings in science news stays relatively steady across different numbers of authors. While team size has been found to be associated with the novelty and impact of science (Lee et al., 2015; Thelwall and Sud, 2016; Sud and Thelwall, 2016), our results indicate that the journalist is largely not influenced by the size of the research team in describing the certainty of their findings.

Across these results, our study suggests that the journalists report scientific findings with less certainty than scientists (RQ1). This result contradicts the existing findings that the journalists are overstating science (Weiss and Singer, 1988; Fahnestock, 2009). The fine-grained analysis over aspect-level certainty provides further details for such a change: journalists may play down the certainty about several core aspects of science findings like SUGGESTION even when they are certain in the abstract (RQ2,3). Moreover, we find that the certainty of scientific findings in research articles varies with journal impact factors and team size, while such a

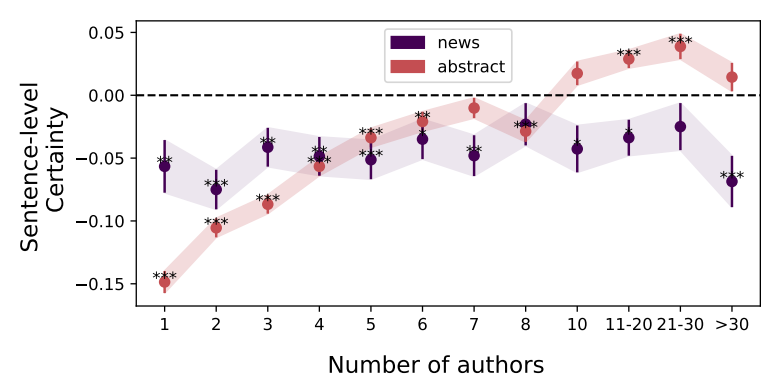

Figure 8: Averaged marginal effects of number of authors on level of certainty. Findings written by more authors are associated with a higher level of certainty, while such a pattern does not persist in science news.

pattern does not persist in science news (RQ4,5), suggesting that journalists may not alter scientific uncertainty according to these factors.

\section{Discussion}

In this paper, we propose a new taxonomy, data, and models for certainty in science communications. Using the model, we analyzed a large scientific finding dataset and answered a series of important research questions on science communications. However, we also note the following limitations of our study. (1) We only use the abstract rather than the full text of research articles due to open access restrictions from copyright. Although authors normally present the core findings in the abstract, findings in abstracts could still be presented differently from findings in the main texts. (2) We use report verbs to extract findings in science news, which may miss findings that are presented without them. How to identify scientific findings in science news is still an open question and we call for future studies in this direction. (3) In our analysis, we use word-based heuristic methods (word overlap and Jaccard similarity) to match findings in news and abstracts, while the same scientific finding can be paraphrased with different sets of words. In future studies, we will develop better methods to identify paraphrases of scientific findings.

\section{Conclusion}

Our study represents a new step towards modeling certainty in text and demonstrates that sentencelevel and aspect-level certainty are natural and feasible ways to model and annotate certainty. The proposed computational framework for certainty in scientific findings could support and inspire new studies on certainty in general language as well as new approaches to study science communication. 


\section{Acknowledgments}

We thank Yumi Kim, Elizabeth Loeher, and Cassie Tian for data annotation. We thank Altmetric.com for sharing science journalism data and Hao Peng for building the preliminary data pipeline. We thank Misha Teplitskiy, Daniel Romero, Jian Zhu, Zuoyu Tian, Yian Yin for their helpful comments. We thank anonymous reviewers and area chairs for their helpful suggestions during the review process. This material is based upon work supported by the National Science Foundation under Grant No. 1850221, the Volkswagen Foundation, and Rackham Graduate Student Research Grant at the University of Michigan.

\section{References}

Hites Ahir, Nicholas Bloom, and Davide Furceri. 2018. The world uncertainty index. Available at SSRN 3275033 .

Eleonore Batteux, Bilovich Avri, Samuel GB Johnson, and David Tuckett. 2021. The negative consequences of failing to communicate uncertainties during a pandemic: The case of covid-19 vaccines. medRxiv.

Iz Beltagy, Kyle Lo, and Arman Cohan. 2019. SciBERT: A pretrained language model for scientific text. In Proceedings of the 2019 Conference on Empirical Methods in Natural Language Processing and the 9th International Joint Conference on Natural Language Processing (EMNLP-IJCNLP), pages 36153620, Hong Kong, China. Association for Computational Linguistics.

Barry Bozeman and Elizabeth Corley. 2004. Scientists' collaboration strategies: implications for scientific and technical human capital. Research policy, 33(4):599-616.

David V Budescu, Stephen Broomell, and Han-Hui Por. 2009. Improving communication of uncertainty in the reports of the intergovernmental panel on climate change. Psychological science, 20(3):299-308.

David V Budescu, Han-Hui Por, and Stephen B Broomell. 2012. Effective communication of uncertainty in the ipcc reports. Climatic change, 113(2):181-200.

Pamela C Corley and Justin Wedeking. 2014. The (dis) advantage of certainty: The importance of certainty in language. Law \& Society Review, 48(1):35-62.

Nathan F Dieckmann, Paul Slovic, and Ellen M Peters. 2009. The use of narrative evidence and explicit likelihood by decisionmakers varying in numeracy. Risk Analysis: An International Journal, 29(10):14731488.
Graham N Dixon and Christopher E Clarke. 2013. Heightening uncertainty around certain science: Media coverage, false balance, and the autism-vaccine controversy. Science Communication, 35(3):358382.

Marek J Druzdzel. 1989. Verbal uncertainty expressions: Literature review. Pittsburgh, PA: Carnegie Mellon University, Department of Engineering and Public Policy, pages 1-13.

Jeanne Fahnestock. 2009. The rhetoric of the natural sciences. The SAGE handbook of rhetorical studies, pages $175-195$.

Richárd Farkas, Veronika Vincze, György Móra, János Csirik, and György Szarvas. 2010. The CoNLL2010 shared task: Learning to detect hedges and their scope in natural language text. In Proceedings of the Fourteenth Conference on Computational Natural Language Learning - Shared Task, pages 1-12, Uppsala, Sweden. Association for Computational Linguistics.

Baruch Fischhoff. 2012. Communicating uncertainty: Fulfilling the duty to inform. Issues in Science and Technology, 28.

Rosanne W Fortner, Jae-Young Lee, Jeffrey R Corney, Samantha Romanello, Joseph Bonnell, Brian Luthy, Claudia Figuerido, and Nyathi Ntsiko. 2000. Public understanding of climate change: Certainty and willingness to act. Environmental Education Research, 6(2):127-141.

Craig R Fox and Gülden Ülkümen. 2011. Distinguishing two dimensions of uncertainty. Perspectives on thinking, judging, and decision making, 14.

Simon French. 1995. Uncertainty and imprecision: modelling and analysis. Journal of the Operational Research Society, 46(1):70-79.

Sharon M Friedman, Sharon Dunwoody, and Carol L Rogers. 1999. Communicating uncertainty: Media coverage of new and controversial science. Routledge.

Abel Gustafson and Ronald E Rice. 2019. The effects of uncertainty frames in three science communication topics. Science Communication, 41(6):679706.

Kara L Hall, Amanda L Vogel, Grace C Huang, Katrina J Serrano, Elise L Rice, Sophia P Tsakraklides, and Stephen M Fiore. 2018. The science of team science: A review of the empirical evidence and research gaps on collaboration in science. American Psychologist, 73(4):532.

Paul KJ Han, William MP Klein, and Neeraj K Arora. 2011. Varieties of uncertainty in health care: a conceptual taxonomy. Medical Decision Making, 31(6):828-838. 
Roderick P Hart and Jay P Childers. 2004. Verbal certainty in american politics: An overview and extension. Presidential Studies Quarterly, 34(3):516535 .

Aron Henriksson and Sumithra Velupillai. 2010. Levels of certainty in knowledge-intensive corpora: an initial annotation study. In Proceedings of the Workshop on Negation and Speculation in Natural Language Processing, pages 41-45, Uppsala, Sweden. University of Antwerp.

Janet Holmes. 1982. Expressing doubt and certainty in english. RELC journal, 13(2):9-28.

Ronald A Howard. 1988. Uncertainty about probability: A decision analysis perspective. Risk Analysis, 8(1):91-98.

Ken Hyland. 1996. Writing without conviction? hedging in science research articles. Applied linguistics, 17(4):433-454.

Ken Hyland. 2005. Metadiscourse: Exploring interaction in writing. Bloomsbury Publishing.

Seongkyoon Jeong and Jae Young Choi. 2015. Collaborative research for academic knowledge creation: How team characteristics, motivation, and processes influence research impact. Science and Public Policy, 42(4):460-473.

Diederik P. Kingma and Jimmy Ba. 2015. Adam: A method for stochastic optimization. In 3rd International Conference on Learning Representations, ICLR 2015, San Diego, CA, USA, May 7-9, 2015, Conference Track Proceedings.

Andrew P Kurmis. 2003. Understanding the limitations of the journal impact factor. JBJS, 85(12):2449-2454.

You-Na Lee, John P Walsh, and Jian Wang. 2015. Creativity in scientific teams: Unpacking novelty and impact. Research policy, 44(3):684-697.

Wei-Hao Lin, Theresa Wilson, Janyce Wiebe, and Alexander Hauptmann. 2006. Which side are you on? identifying perspectives at the document and sentence levels. In Proceedings of the Tenth Conference on Computational Natural Language Learning (CoNLL-X), pages 109-116, New York City. Association for Computational Linguistics.

Yinhan Liu, Myle Ott, Naman Goyal, Jingfei Du, Mandar Joshi, Danqi Chen, Omer Levy, Mike Lewis, Luke Zettlemoyer, and Veselin Stoyanov. 2019. Roberta: A robustly optimized bert pretraining approach. arXiv preprint arXiv:1907.11692.

Donald MacKenzie. 1998. The certainty trough. In Exploring expertise, pages 325-329. Springer.

Arun Meena and TV Prabhakar. 2007. Sentence level sentiment analysis in the presence of conjuncts using linguistic analysis. In European conference on information retrieval, pages 573-580. Springer.
Ali Mosleh and Vicki M Bier. 1996. Uncertainty about probability: a reconciliation with the subjectivist viewpoint. IEEE Transactions on Systems, Man, and Cybernetics-Part A: Systems and Humans, 26(3):303-310.

National Academies of Sciences, Engineering, and Medicine. 2017. Communicating science effectively: A research agenda.

Fabian Pedregosa, Gaël Varoquaux, Alexandre Gramfort, Vincent Michel, Bertrand Thirion, Olivier Grisel, Mathieu Blondel, Peter Prettenhofer, Ron Weiss, Vincent Dubourg, et al. 2011. Scikit-learn: Machine learning in python. the Journal of machine Learning research, 12:2825-2830.

Ellen Peters, P Sol Hart, Martin Tusler, and Liana Fraenkel. 2014a. Numbers matter to informed patient choices: a randomized design across age and numeracy levels. Medical Decision Making, 34(4):430-442.

Ellen Peters, Louise Meilleur, and Mary Kate Tompkins. 2014b. Numeracy and the affordable care act: Opportunities and challenges. In Health literacy and numeracy: Workshop summary. National Academies Press (US).

Katherine W Phillips, Nancy P Rothbard, and Tracy L Dumas. 2009. To disclose or not to disclose? status distance and self-disclosure in diverse environments. Academy of Management Review, 34(4):710-732.

Mary C Politi, Paul KJ Han, and Nananda F Col. 2007. Communicating the uncertainty of harms and benefits of medical interventions. Medical Decision Making, 27(5):681-695.

Vinodkumar Prabhakaran, William L. Hamilton, Dan McFarland, and Dan Jurafsky. 2016. Predicting the rise and fall of scientific topics from trends in their rhetorical framing. In Proceedings of the 54th Annual Meeting of the Association for Computational Linguistics (Volume 1: Long Papers), pages 11701180, Berlin, Germany. Association for Computational Linguistics.

Publons. 2018. The 2018 global state of peer review. https://publons.com/community/gspr.

Marta Recasens, Cristian Danescu-Niculescu-Mizil, and Dan Jurafsky. 2013. Linguistic models for analyzing and detecting biased language. In Proceedings of the 51st Annual Meeting of the Association for Computational Linguistics (Volume 1: Long Papers), pages 1650-1659, Sofia, Bulgaria. Association for Computational Linguistics.

Victoria L. Rubin. 2007. Stating with certainty or stating with doubt: Intercoder reliability results for manual annotation of epistemically modalized statements. In Human Language Technologies 2007: The Conference of the North American Chapter of the Association for Computational Linguistics; Companion Volume, Short Papers, pages 141-144, 
Rochester, New York. Association for Computational Linguistics.

Victoria L Rubin, Elizabeth D Liddy, and Noriko Kando. 2006. Certainty identification in texts: Categorization model and manual tagging results. In Computing attitude and affect in text: Theory and applications, pages 61-76. Springer.

Somnath Saha, Sanjay Saint, and Dimitri A Christakis. 2003. Impact factor: a valid measure of journal quality? Journal of the Medical Library Association, 91(1):42.

Roser Saurí and James Pustejovsky. 2009. Factbank: a corpus annotated with event factuality. Language resources and evaluation, 43(3):227-268.

Kim Schouten and Flavius Frasincar. 2015. Survey on aspect-level sentiment analysis. IEEE Transactions on Knowledge and Data Engineering, 28(3):813830 .

Aleksandr Sinayev, Ellen Peters, Martin Tusler, and Liana Fraenkel. 2015. Presenting numeric information with percentages and descriptive risk labels: a randomized trial. Medical decision making, 35(8):937-947.

Michael Smithson. 2012. Ignorance and uncertainty: Emerging paradigms. Springer Science \& Business Media.

Sandeep Soni, Tanushree Mitra, Eric Gilbert, and Jacob Eisenstein. 2014. Modeling factuality judgments in social media text. In Proceedings of the 52nd Annual Meeting of the Association for Computational Linguistics (Volume 2: Short Papers), pages 415420, Baltimore, Maryland. Association for Computational Linguistics.

Daniel Stokols, Kara L Hall, Brandie K Taylor, and Richard P Moser. 2008. The science of team science: overview of the field and introduction to the supplement. American journal of preventive medicine, 35(2):S77-S89.

Pardeep Sud and Mike Thelwall. 2016. Not all international collaboration is beneficial: The $\mathrm{m}$ endeley readership and citation impact of biochemical research collaboration. Journal of the Association for Information Science and Technology, 67(8):1849_ 1857.

György Szarvas, Veronika Vincze, Richárd Farkas, György Móra, and Iryna Gurevych. 2012. Crossgenre and cross-domain detection of semantic uncertainty. Computational Linguistics, 38(2):335-367.

Yla R Tausczik and James W Pennebaker. 2010. The psychological meaning of words: Liwc and computerized text analysis methods. Journal of language and social psychology, 29(1):24-54.
Mike Thelwall and Pardeep Sud. 2016. Mendeley readership counts: An investigation of temporal and disciplinary differences. Journal of the Association for Information Science and Technology, 67(12):30363050 .

Daniel P Thunnissen. 2003. Uncertainty classification for the design and development of complex systems. In $3 r d$ annual predictive methods conference, pages 1-16. Newport Beach CA.

Anne Marthe Van Der Bles, Sander van der Linden, Alexandra LJ Freeman, and David J Spiegelhalter. 2020. The effects of communicating uncertainty on public trust in facts and numbers. Proceedings of the National Academy of Sciences, 117(14):7672-7683.

Eva Thue Vold. 2006. Epistemic modality markers in research articles: a cross-linguistic and crossdisciplinary study. International Journal of Applied Linguistics, 16(1):61-87.

Kuansan Wang, Zhihong Shen, Chiyuan Huang, ChiehHan Wu, Darrin Eide, Yuxiao Dong, Junjie Qian, Anshul Kanakia, Alvin Chen, and Richard Rogahn. 2019. A review of microsoft academic services for science of science studies. Frontiers in Big Data, 2:45.

Carol Weiss and Eleanor Singer. 1988. Reporting of social science in the national media. Russell Sage Foundation.

Amanda Wilson, Jane Robertson, Patrick McElduff, Alison Jones, and David Henry. 2010. Does it matter who writes medical news stories? PLOS medicine, 7(9):e1000323.

Paul D Windschitl and Gary L Wells. 1996. Measuring psychological uncertainty: Verbal versus numeric methods. Journal of Experimental Psychology: Applied, 2(4):343.

Thomas Wolf, Julien Chaumond, Lysandre Debut, Victor Sanh, Clement Delangue, Anthony Moi, Pierric Cistac, Morgan Funtowicz, Joe Davison, Sam Shleifer, et al. 2020. Transformers: State-of-theart natural language processing. In Proceedings of the 2020 Conference on Empirical Methods in Natural Language Processing: System Demonstrations, pages 38-45.

Wendy Wood and Alice H Eagly. 2009. Advantages of certainty and uncertainty. The handbook of research synthesis and meta-analysis, 2:455-472.

Lingfei Wu, Dashun Wang, and James A Evans. 2019. Large teams develop and small teams disrupt science and technology. Nature, 566(7744):378-382. 


\section{A Data Preprocessing}

Altmetric mention data Altmetric ${ }^{3}$ tracks a variety of sources for mentions of research papers, including coverage from over 2,000 news outlets around the world. To control for differences in the frequency of scientific reporting and potential confounds from variations in journalistic practices across different countries, the list of news outlets was curated to 423 U.S.-based news media outlets, with each having at least 1,000 mentions in the Altmetric database. Location data for each outlet is provided by Altmetric. This initial dataset consists of $2.4 \mathrm{M}$ mentions of $521 \mathrm{~K}$ papers by $1.7 \mathrm{M}$ news articles before 2019-10-06. Each mention in the Altmetric data has associated metadata that allows us to retrieve the original citing news story as well as the DOI for the paper itself.

News processing During data processing, we notice that some very long news articles are usually policy documents. Therefore, we removed news longer than 1392 words (top 5\%). To ensure that each news is specifically written about a single research paper's findings, we keep news only linked to one research paper. This leads to 128,942 newspaper pairs spanning 273 different news outlets and 57,807 different scientific articles. For all the news stories, we first remove references and paragraphs containing quotes as they might bias our analysis of uncertainty (e.g., a scientist describes their own work as uncertain in a quote).

\section{B Model Details}

We use scikit-learn version 0.23 .1 to build the linear regression model (Pedregosa et al., 2011). Specifically, for the linear model, we use ridge regressor and classifier with default settings. The built-in CountVectorizer of scikit-learn is used to vectorize the unigram, bigram, and trigram of each input question. The size of the bag-of-words feature vector is set as 40000 .

For both the SciBERT and RoBERTa models, we use Hugging Face ${ }^{4}$ transformers (Wolf et al., 2020) and set the batch size as 128 and learning rate as 0.0001 . We set max_len=60. Adam (Kingma and $\mathrm{Ba}, 2015$ ) is used for optimization. All the other hyperparameters and the model size are the same as the default roberta-base model and the SCIBERT model. We train both models for 50 epochs and choose the model with the lowest loss on the validation set. All the code, datasets, and parameters of our best-performing model are released and one could easily reproduce all the experiments.

\section{Additional Details on Extracting Scientific Findings}

We use the following lexicons to extract scientific findings in news:

found that, find that, finds that, reveal that, reveals that, revealed that, suggest that, suggested that, suggests that, discover that, discovers that, discovered that, show that, shows that, showed that, conclude that, concludes that, concluded that, indicate that, indicates that, indicated that, claim that, claims that, claimed that, argue that, argues that, argued that

We manually annotate 50 extracted findings and only 1 of them does not fully counted as a scientific finding, indicating high precision of our approach. Table 4 presents the extracted findings from news and abstract.

\section{Annotation agreement}

Figure 10 presents the Krippendorff's $\alpha$ for aspectlevel certainty annotation.

\section{E Annotated data}

Figure 11 presents the distribution of aspect-level certainty score in annotated dataset. Figure 9 presents the distribution of sentence-level certainty across data splits. Table 2 and Table 3 presents the annotated findings for sentence-level and aspectlevel certainty.

\section{F Matched finding samples}

Table 5 presents the samples for matched findings.

\section{G Detailed regression results}

Table 6 presents the regression results for RQ1. Table 7 presents the regression results for RQ2. Table 8 presents the regression results for RQ3. Table 9 and Table 10 present the regression results for RQ4-5.

\footnotetext{
${ }^{3}$ https://www.altmetric.com/

${ }^{4}$ https://huggingface.co/
} 


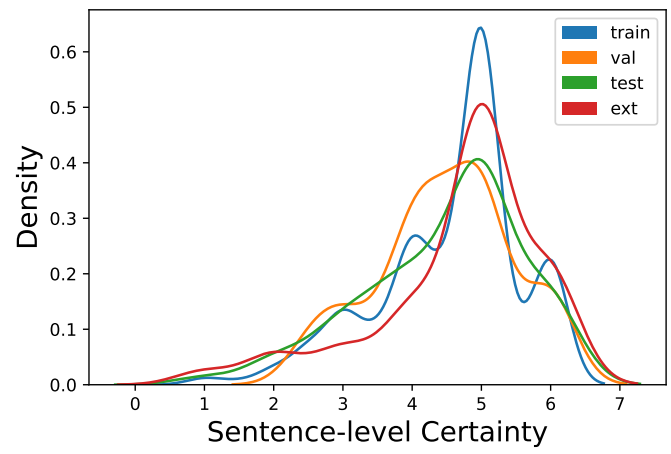

Figure 9: The distribution of sentence-level certainty score across the annotated dataset

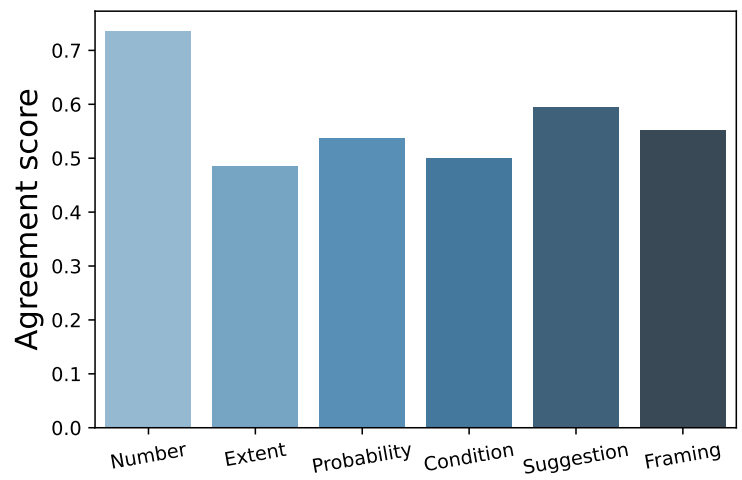

Figure 10: Agreement scores for annotating aspectlevel certainty

\begin{tabular}{c|l|c}
\hline \# Hedges & Text & Score \\
\hline 4 & $\begin{array}{l}\text { Based on these observations, we pro- } \\
\text { pose that the apparent receding contact } \\
\text { angle should be used for characterizing } \\
\text { superliquid-repellent surfaces rather than } \\
\text { the apparent advancing contact angle and } \\
\text { hysteresis. } \\
\text { The nondemented subjects with Alzheimer } \\
\text { pathology may have had "preclinical” AD, } \\
\text { or numerous cortical plaques may occur in } \\
\text { some elderly subjects who would never de- } \\
\text { velop clinical dementia. }\end{array}$ & 4.0 \\
2 & $\begin{array}{l}\text { These mouthwashes may be of modest ben- } \\
\text { efit, it is unclear if a certain subset of pa- } \\
\text { tients showed a large response while others } \\
\text { derived no benefit. } \\
\text { Circadian rhythm disruptions, such as jet } \\
\text { lag, might be linked with an increased risk } \\
\text { of cancer, she said. } \\
\text { Further research is necessary to understand } \\
\text { whether this is a causal relationship. }\end{array}$ & 2.0 \\
\hline
\end{tabular}

Table 2: Annotated sentence-level certainty for findings with different numbers of hedges. The number of hedges does not necessarily reflect the overall perception of certainty. 

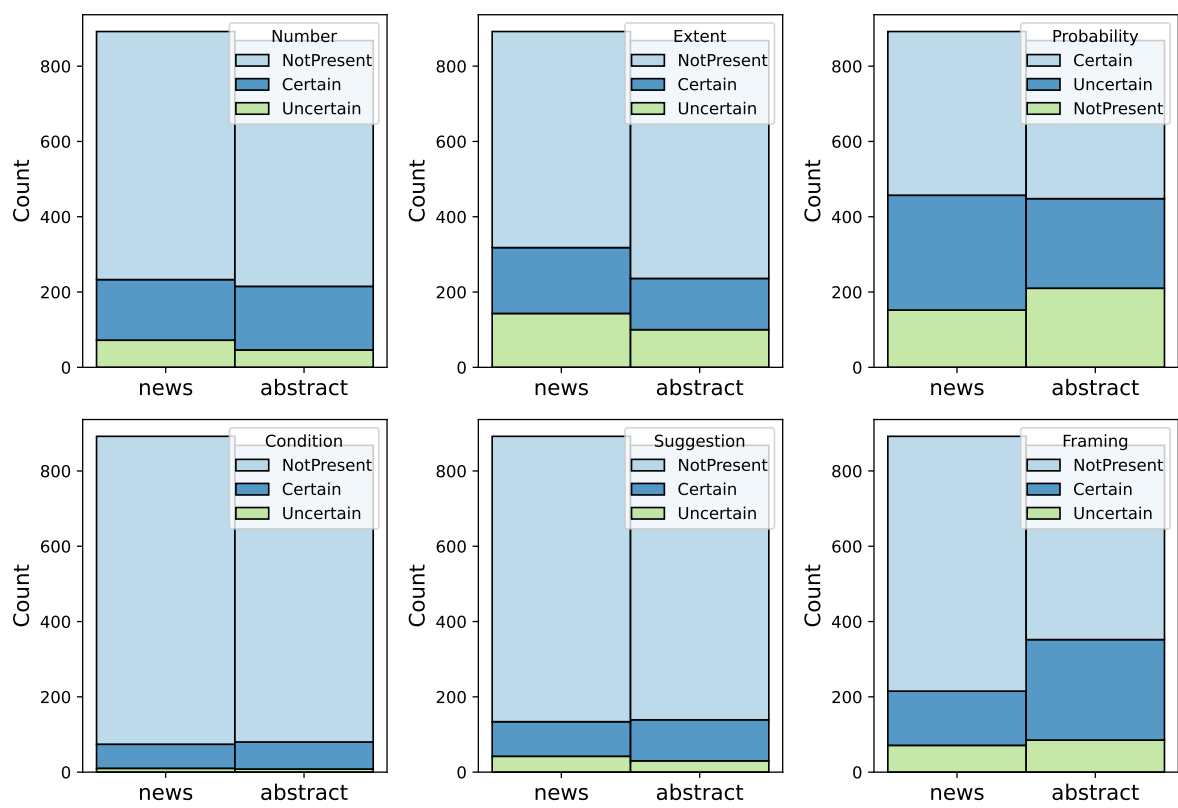

Figure 11: The distribution of aspect-level certainty scores in the annotated dataset

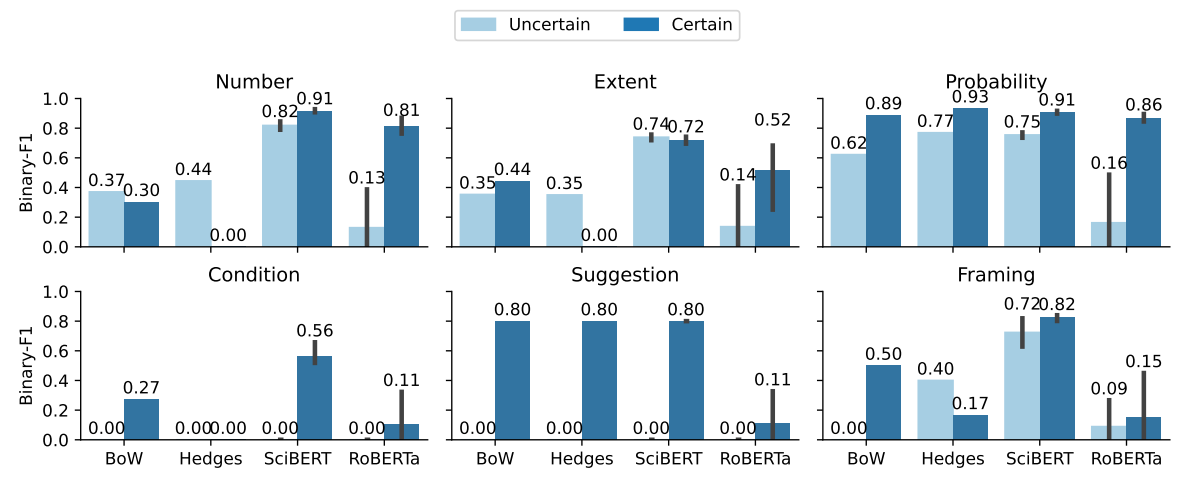

Figure 12: Aspect-level certainty prediction results over the random sample.

\begin{tabular}{l|l|l|}
\hline Finding & Uncertain & Certain \\
\hline $\begin{array}{l}\text { Arctic sea ice is declining at a rate of nearly 13 } \\
\text { percent per decade. }\end{array}$ & $\begin{array}{l}\text { NUMBER, EX- } \\
\text { TENT }\end{array}$ & PROBABILITY \\
\hline $\begin{array}{l}\text { Some functions show decreases with potentially } \\
\text { irreversible global impacts }\end{array}$ & $\begin{array}{l}\text { EXTENT, } \\
\text { PROBABILITY }\end{array}$ & \\
\hline $\begin{array}{l}\text { There were 365 cases of maternal sepsis, giving } \\
\text { an incidence of severe maternal sepsis of 4.7 } \\
\text { women per 100,000. }\end{array}$ & & $\begin{array}{l}\text { NUMBER, EX- } \\
\text { TENT }\end{array}$ \\
\hline $\begin{array}{l}\text { The practice may actually drive away qualified } \\
\text { applicants who feel that their privacy has been } \\
\text { compromised. }\end{array}$ & PROBABILITY & \\
\hline $\begin{array}{l}\text { More research is needed on how cryptic post- } \\
\text { copulatory and post-zygotic processes con- } \\
\text { tribute to determining paternity and bridging } \\
\text { the behavioural and genetic mating systems of } \\
\text { viviparous species. }\end{array}$ & PROBABILITY & SUGGESTION \\
\hline \hline
\end{tabular}

Table 3: Annotated aspect-level certainty for sampled findings 


\begin{tabular}{|c|c|c|c|}
\hline Original text & Extracted finding & Keyword & Source \\
\hline $\begin{array}{l}\text { In addition, liver procurement data such as WIT } \\
\text { showed that organs with less than } 30 \text { mins WIT } \\
\text { led to significantly reduced yield, but no impact } \\
\text { was found on viability. }\end{array}$ & $\begin{array}{l}\text { Organs with less than } 30 \text { mins wit led to signifi- } \\
\text { cantly reduced yield, but no impact was found } \\
\text { on viability. }\end{array}$ & showed & news \\
\hline $\begin{array}{l}\text { I conclude that we live in one of infinitely many } \\
\text { universes - one for each value of the gravitational } \\
\text { constant. }\end{array}$ & $\begin{array}{l}\text { We live in one of infinitely many universes - one } \\
\text { for each value of the gravitational constant. }\end{array}$ & conclude & news \\
\hline $\begin{array}{l}\text { They found that the findings were specific to } \\
\text { ADHD, with no associations observed between } \\
\text { the other two disorders. }\end{array}$ & $\begin{array}{l}\text { The findings were specific to adhd, with no as- } \\
\text { sociations observed between the other two disor- } \\
\text { ders. }\end{array}$ & found & news \\
\hline $\begin{array}{l}\text { The Irish investigators of that meta-analysis } \\
\text { found that methotrexate was associated with a } \\
\text { small albeit statistically significant } 10 \% \text { increase } \\
\text { in the risk of all adverse respiratory events and } \\
\text { an } 11 \% \text { increase in the risk of respiratory infec- } \\
\text { tion. }\end{array}$ & $\begin{array}{l}\text { Methotrexate was associated with a small albeit } \\
\text { statistically significant } 10 \% \text { increase in the risk } \\
\text { of all adverse respiratory events and an } 11 \% \\
\text { increase in the risk of respiratory infection. }\end{array}$ & found & news \\
\hline $\begin{array}{l}\text { The study, in the current issue of Research in } \\
\text { Nursing \& Health, revealed that while physical } \\
\text { environment had no direct influence on job sat- } \\
\text { isfaction, it did have a significant indirect influ- } \\
\text { ence because the environment affected whether } \\
\text { nurses could complete tasks without interrup- } \\
\text { tions, communicate easily with other nurses and } \\
\text { physicians, and/or do their jobs efficiently. }\end{array}$ & $\begin{array}{l}\text { While physical environment had no direct in- } \\
\text { fluence on job satisfaction, it did have a signifi- } \\
\text { cant indirect influence because the environment } \\
\text { affected whether nurses could complete tasks } \\
\text { without interruptions, communicate easily with } \\
\text { other nurses and physicians, and/or do their jobs } \\
\text { efficiently. }\end{array}$ & revealed & news \\
\hline $\begin{array}{l}\text { Mixed-species neighbourhoods did not signifi- } \\
\text { cantly affect tree ring growth in normal years. }\end{array}$ & $\begin{array}{l}\text { Mixed-species neighbourhoods did not signifi- } \\
\text { cantly affect tree ring growth in normal years. }\end{array}$ & & abstract \\
\hline $\begin{array}{l}\text { Statistical tests (ordinary least squares, quantile, } \\
\text { robust regressions, Akaike information criterion } \\
\text { model tests) document independence from phy- } \\
\text { logeny, and a previously unrecognized strong } \\
\text { and significant correlation of } \sigma 13 \mathrm{C} \text { enrichment } \\
\text { with body mass for all mammalian herbivores. }\end{array}$ & $\begin{array}{l}\text { Statistical tests (ordinary least squares, quantile, } \\
\text { robust regressions, Akaike information criterion } \\
\text { model tests) document independence from phy- } \\
\text { logeny, and a previously unrecognized strong } \\
\text { and significant correlation of } \sigma 13 \mathrm{C} \text { enrichment } \\
\text { with body mass for all mammalian herbivores. }\end{array}$ & & abstract \\
\hline $\begin{array}{l}\text { There were no differences in socioeconomic sta- } \\
\text { tus, cognitive reserve, general cognitive status, } \\
\text { or lipid and TSH profiles between the groups. }\end{array}$ & $\begin{array}{l}\text { There were no differences in socioeconomic sta- } \\
\text { tus, cognitive reserve, general cognitive status, } \\
\text { or lipid and TSH profiles between the groups. }\end{array}$ & & abstract \\
\hline $\begin{array}{l}\text { Much remains unknown and multiple research } \\
\text { disciplines are needed to address this: forest } \\
\text { scientists and other biologists have a major role } \\
\text { to play. }\end{array}$ & $\begin{array}{l}\text { Much remains unknown and multiple research } \\
\text { disciplines are needed to address this: forest } \\
\text { scientists and other biologists have a major role } \\
\text { to play. }\end{array}$ & & abstract \\
\hline $\begin{array}{l}\text { The co-administration of the energy drink with } \\
\text { alcohol did not alter the alcohol-induced impair- } \\
\text { ment on these objective measures. }\end{array}$ & $\begin{array}{l}\text { The co-administration of the energy drink with } \\
\text { alcohol did not alter the alcohol-induced impair- } \\
\text { ment on these objective measures. }\end{array}$ & & abstract \\
\hline
\end{tabular}

Table 4: Extracted findings from news and abstracts 


\begin{tabular}{|c|c|c|c|}
\hline News finding & Abstract finding & $\begin{array}{l}\text { Jaccard } \\
\text { Simi- } \\
\text { larity }\end{array}$ & $\begin{array}{l}\text { Word } \\
\text { Over- } \\
\text { lap }\end{array}$ \\
\hline $\begin{array}{l}\text { For children with low self-esteem, high praise } \\
\text { may be more harmful than helpful. }\end{array}$ & $\begin{array}{l}\text { Inflated praise decreases challenge seeking in } \\
\text { children with low self-esteem and has the oppo- } \\
\text { site effect on children with high self-esteem. }\end{array}$ & 0.36 & 5 \\
\hline $\begin{array}{l}\text { Breast-feeding might be no more beneficial than } \\
\text { bottle-feeding for } 10 \text { of } 11 \text { long-term health and } \\
\text { well-being outcomes in children age } 4 \text { to } 14 \text {. }\end{array}$ & $\begin{array}{l}\text { Children aged } 4 \text { to } 14 \text { who were breast- as op- } \\
\text { posed to bottle-fed did significantly better on } 10 \\
\text { of the } 11 \text { outcomes studied. }\end{array}$ & 0.35 & 7 \\
\hline $\begin{array}{l}\text { While our first impressions of educators might } \\
\text { affect our ratings of them, ultimately the quality } \\
\text { of their instruction matters the most in student } \\
\text { evaluations. }\end{array}$ & $\begin{array}{l}\text { Quality of instruction is the strongest determi- } \\
\text { nant of student factual and conceptual learning, } \\
\text { but that both instructional quality and first im- } \\
\text { pressions affect evaluations of the instructor. }\end{array}$ & 0.39 & 7 \\
\hline $\begin{array}{l}\text { Even in the absence of symptoms, trauma may } \\
\text { have an enduring effect on brain function. }\end{array}$ & $\begin{array}{l}\text { Trauma has a measurable, enduring effect upon } \\
\text { the functional dynamics of the brain, even in } \\
\text { individuals who experience trauma but do not } \\
\text { develop ptsd. }\end{array}$ & 0.38 & 6 \\
\hline $\begin{array}{l}\text { Being bullied may increase the risk for parasom- } \\
\text { nias. }\end{array}$ & $\begin{array}{l}\text { Being bullied increases the risk for having para- } \\
\text { somnias. }\end{array}$ & 0.67 & 4 \\
\hline $\begin{array}{l}\text { Dried fruits may lower the gi of white bread } \\
\text { through displacement of high gi carbohydrate. }\end{array}$ & $\begin{array}{l}\text { When displacing half the available carbohydrate } \\
\text { in white bread, all dried fruit lowered the GI; } \\
\text { however, only dried apricots }\left(G I_{-}=-57\right. \\
\text { showed a significant displacement effect }\left(P_{-}=\right. \\
0.025) \text {. }\end{array}$ & 0.36 & 8 \\
\hline $\begin{array}{l}\text { Although the biological mechanisms of these } \\
\text { associations need to be explored in future re- } \\
\text { search, these new data may shed new light on } \\
\text { the long-observed epidemiological associations } \\
\text { between personality, physical health, and human } \\
\text { longevity. }\end{array}$ & $\begin{array}{l}\text { The present data shed new light on the } \\
\text { long-observed epidemiological associations be- } \\
\text { tween personality, physical health, and human } \\
\text { longevity. }\end{array}$ & 0.57 & 12 \\
\hline $\begin{array}{l}\text { Late colonies more frequently rejected both } \\
\text { young and old non-nestmates, suggesting that } \\
\text { risk of acceptance may be too high at this stage. }\end{array}$ & $\begin{array}{l}\text { Young non-nestmates were more frequently ac- } \\
\text { cepted in early than in late colonies. }\end{array}$ & 0.43 & 6 \\
\hline $\begin{array}{l}\text { Only graphic warning labels reduced the per- } \\
\text { centage of sugary drinks purchased, and that the } \\
\text { public may support the use of graphic labels if } \\
\text { they are informed that only graphic labels are } \\
\text { effective. }\end{array}$ & $\begin{array}{l}\text { Graphic warning labels reduced the share of sug- } \\
\text { ary drinks purchased in a cafeteria from } 21.4 \% \\
\text { at baseline to } 18.2 \% \text { effect driven by substitution } \\
\text { of water for sugary drinks. }\end{array}$ & 0.36 & 8 \\
\hline $\begin{array}{l}\text { The kenyan runners are able to maintain their } \\
\text { cerebral oxygenation within a stable range, } \\
\text { which may contribute to their success in long- } \\
\text { distance races. }\end{array}$ & $\begin{array}{l}\text { Kenyan runners from the kalenjin tribe are able } \\
\text { to maintain their cerebral oxygenation within a } \\
\text { stable range during a self-paced maximal } 5-\mathrm{km} \\
\text { time trial, but not during an incremental maximal } \\
\text { test. }\end{array}$ & 0.39 & 9 \\
\hline $\begin{array}{l}\text { The world might be closer to exceeding the bud- } \\
\text { get for the long-term target of the paris climate } \\
\text { agreement than previously thought. }\end{array}$ & $\begin{array}{l}\text { The world is closer to exceeding the budget for } \\
\text { the long-term target of the paris climate agree- } \\
\text { ment than previously thought. }\end{array}$ & 0.92 & 11 \\
\hline $\begin{array}{l}\text { Src may be associated with longer overall sur- } \\
\text { vival. }\end{array}$ & $\begin{array}{l}\text { Higher src activity is associated with longer over- } \\
\text { all survival. }\end{array}$ & 0.625 & 5 \\
\hline $\begin{array}{l}\text { Pg-free mel may not reduce short-term compli- } \\
\text { cations or improve outcomes after asct for mm. }\end{array}$ & $\begin{array}{l}\text { In summary, we demonstrate that switching to } \\
\text { PG-free MEL did not significantly reduce short- } \\
\text { term complications of ASCT or improve out- } \\
\text { comes in MM. }\end{array}$ & 0.64 & 9 \\
\hline $\begin{array}{l}\text { Higher adenoma detection rates may be associ- } \\
\text { ated with up to } 50 \text { percent to } 60 \text { percent lower } \\
\text { lifetime colorectal cancer incidence and death } \\
\text { without higher overall costs, despite a higher } \\
\text { number of colonoscopies and potential compli- } \\
\text { cations, according to a study in the june } 16 \text { issue } \\
\text { of jama. }\end{array}$ & $\begin{array}{l}\text { In this microsimulation modeling study, } \\
\text { higher adenoma detection rates in screening } \\
\text { colonoscopy were associated with lower lifetime } \\
\text { risks of colorectal cancer and colorectal cancer } \\
\text { mortality without being associated with higher } \\
\text { overall costs. }\end{array}$ & 0.39 & 14 \\
\hline $\begin{array}{l}\text { Long-term ppi use may increase the risk of hip } \\
\text { fracture. }\end{array}$ & $\begin{array}{l}\text { The increased risk of hip fracture was evident } \\
\text { only in short-term proton pump inhibitor use, } \\
\text { but no association was found for long-term or } \\
\text { cumulative use. }\end{array}$ & 0.38 & 6 \\
\hline
\end{tabular}

Table 5: Matched findings from news and abstracts 


\begin{tabular}{|c|c|c|c|c|c|}
\hline & index & coeff & SE & $\mathrm{p}$ value & \\
\hline 0 & Intercept & $4.611216 \mathrm{e}+00$ & $7.331098 \mathrm{e}-02$ & $0.000000 \mathrm{e}+00$ & $* * *$ \\
\hline 1 & source[T.news] & $-3.152504 \mathrm{e}-02$ & $1.030003 \mathrm{e}-02$ & $2.212871 \mathrm{e}-03$ & $* *$ \\
\hline 2 & finding_len & $-3.606883 e-03$ & $5.813032 \mathrm{e}-04$ & $5.641478 \mathrm{e}-10$ & $* * *$ \\
\hline 3 & flesch_reading_ease & $-8.410694 \mathrm{e}-04$ & $2.318612 \mathrm{e}-04$ & $2.873043 \mathrm{e}-04$ & $* * *$ \\
\hline 4 & first_author_rank & $6.160764 \mathrm{e}-06$ & $2.968990 \mathrm{e}-06$ & $3.800262 \mathrm{e}-02$ & $*$ \\
\hline 5 & affi_rank & $4.904435 \mathrm{e}-06$ & $2.961068 \mathrm{e}-06$ & $9.768389 \mathrm{e}-02$ & \\
\hline 6 & journal_impact & $1.831817 \mathrm{e}-03$ & $4.162222 \mathrm{e}-04$ & $1.085809 \mathrm{e}-05$ & $* * *$ \\
\hline 7 & num_authors & $1.120942 \mathrm{e}-03$ & $4.036951 \mathrm{e}-04$ & $5.499123 \mathrm{e}-03$ & $* *$ \\
\hline 8 & Law & $-4.923866 \mathrm{e}-01$ & $2.018834 \mathrm{e}-01$ & $1.474259 \mathrm{e}-02$ & $*$ \\
\hline 9 & Condensed_matter_physics & $-4.078086 \mathrm{e}-01$ & $4.113205 \mathrm{e}-01$ & $3.214785 \mathrm{e}-01$ & \\
\hline 10 & Biomedical_engineering & $4.695178 \mathrm{e}-01$ & $3.885994 \mathrm{e}-01$ & $2.269804 \mathrm{e}-01$ & \\
\hline 11 & Hydrology & $4.014678 \mathrm{e}-01$ & $3.408326 \mathrm{e}-01$ & $2.388568 \mathrm{e}-01$ & \\
\hline 12 & Atomic_physics & $3.464856 \mathrm{e}-01$ & $4.837765 \mathrm{e}-01$ & $4.738745 \mathrm{e}-01$ & \\
\hline 13 & Development_economics & $6.620255 \mathrm{e}-01$ & $5.505248 \mathrm{e}-01$ & $2.291780 \mathrm{e}-01$ & \\
\hline 14 & Optics & 7.390099e-02 & $6.006107 \mathrm{e}-01$ & $9.020749 \mathrm{e}-01$ & \\
\hline 15 & Public_policy & $4.924301 \mathrm{e}-01$ & $3.695244 \mathrm{e}-01$ & $1.826846 \mathrm{e}-01$ & \\
\hline 16 & Lung_cancer & $-1.486488 \mathrm{e}-01$ & $8.772435 \mathrm{e}-02$ & $9.019439 \mathrm{e}-02$ & \\
\hline 17 & Electronic_engineering & $1.039881 \mathrm{e}+00$ & $1.009401 \mathrm{e}+00$ & $3.029366 \mathrm{e}-01$ & \\
\hline 18 & Artificial_intelligence & $5.493533 \mathrm{e}-01$ & $5.104198 \mathrm{e}-01$ & $2.818231 \mathrm{e}-01$ & \\
\hline 19 & Machine_learning & $-2.278651 \mathrm{e}+00$ & $5.406896 \mathrm{e}-01$ & $2.521916 \mathrm{e}-05$ & $* * *$ \\
\hline 20 & Gender_studies & $6.166049 \mathrm{e}-01$ & $3.250722 \mathrm{e}-01$ & $5.787315 \mathrm{e}-02$ & \\
\hline 21 & Chemical_engineering & $4.877528 \mathrm{e}-01$ & $3.794780 \mathrm{e}-01$ & $1.987013 \mathrm{e}-01$ & \\
\hline 22 & Biophysics & $-1.122923 \mathrm{e}-01$ & $2.328199 \mathrm{e}-01$ & $6.295908 \mathrm{e}-01$ & \\
\hline 23 & Analytical_chemistry & $-2.179638 \mathrm{e}-02$ & $4.128484 \mathrm{e}-01$ & $9.578960 \mathrm{e}-01$ & \\
\hline 24 & Computer_science & $-2.618102 \mathrm{e}-02$ & $2.719240 \mathrm{e}-01$ & $9.232991 \mathrm{e}-01$ & \\
\hline 25 & Radiology & $2.196766 \mathrm{e}-01$ & $9.690635 \mathrm{e}-02$ & $2.341285 \mathrm{e}-02$ & $*$ \\
\hline 26 & Urology & $6.425612 \mathrm{e}-01$ & $1.465147 \mathrm{e}-01$ & $1.165494 \mathrm{e}-05$ & $* * *$ \\
\hline 27 & Architecture & $-1.176468 \mathrm{e}-01$ & $3.326552 \mathrm{e}-01$ & $7.235995 \mathrm{e}-01$ & \\
\hline 28 & Quantum_mechanics & $4.168358 \mathrm{e}-01$ & $1.317573 \mathrm{e}+00$ & $7.517295 \mathrm{e}-01$ & \\
\hline 29 & Particle_physics & $-1.460230 \mathrm{e}+00$ & $1.140169 \mathrm{e}+00$ & $2.003172 \mathrm{e}-01$ & \\
\hline 30 & Materials_science & $2.811691 \mathrm{e}-02$ & $1.965188 \mathrm{e}-01$ & $8.862333 \mathrm{e}-01$ & \\
\hline 31 & Computational_biology & $-4.444091 \mathrm{e}-01$ & $4.838163 e-01$ & $3.583483 \mathrm{e}-01$ & \\
\hline 32 & Agronomy & $-8.468680 \mathrm{e}-01$ & $2.590309 \mathrm{e}-01$ & $1.080660 \mathrm{e}-03$ & $* *$ \\
\hline 33 & Environmental_resource_management & $-1.147462 \mathrm{e}-01$ & $1.494174 \mathrm{e}-01$ & $4.425267 \mathrm{e}-01$ & \\
\hline 34 & Molecular_biology & $3.467210 \mathrm{e}-01$ & $9.677645 \mathrm{e}-02$ & $3.412987 \mathrm{e}-04$ & $* * *$ \\
\hline 35 & Dermatology & $4.811503 e-01$ & $1.318250 \mathrm{e}-01$ & $2.633475 \mathrm{e}-04$ & $* * *$ \\
\hline 36 & Engineering & $-8.098552 \mathrm{e}-02$ & $3.509610 \mathrm{e}-01$ & $8.175098 \mathrm{e}-01$ & \\
\hline 37 & Surgery & $2.220879 \mathrm{e}-01$ & $4.921727 \mathrm{e}-02$ & $6.465843 \mathrm{e}-06$ & $* * *$ \\
\hline 38 & Public_health & $-2.742082 \mathrm{e}-01$ & $6.707385 \mathrm{e}-02$ & $4.374232 \mathrm{e}-05$ & $* * *$ \\
\hline 39 & Molecular_physics & $-1.015549 e+00$ & $4.841792 \mathrm{e}-01$ & $3.597167 \mathrm{e}-02$ & $*$ \\
\hline 40 & Bioinformatics & 4.398066e-01 & $2.218647 \mathrm{e}-01$ & $4.746451 \mathrm{e}-02$ & $*$ \\
\hline 41 & Physics & $3.112521 \mathrm{e}-01$ & $2.519723 \mathrm{e}-01$ & $2.167548 \mathrm{e}-01$ & \\
\hline 42 & Cognitive_psychology & $-3.977321 \mathrm{e}-03$ & $1.263384 \mathrm{e}-01$ & $9.748860 \mathrm{e}-01$ & \\
\hline 43 & Oncology & $1.807840 \mathrm{e}-01$ & 8.691933e-02 & $3.755370 \mathrm{e}-02$ & $*$ \\
\hline 44 & Pathology & $-1.228369 \mathrm{e}-01$ & $9.014961 \mathrm{e}-02$ & $1.730356 \mathrm{e}-01$ & \\
\hline 45 & Biochemistry & $-1.430021 \mathrm{e}-01$ & $1.234545 \mathrm{e}-01$ & $2.467472 \mathrm{e}-01$ & \\
\hline 46 & Social_science & $-9.696063 e-02$ & $4.913855 \mathrm{e}-01$ & $8.435795 \mathrm{e}-01$ & \\
\hline 47 & Climatology & $-1.757612 \mathrm{e}-01$ & $1.364930 \mathrm{e}-01$ & $1.978755 \mathrm{e}-01$ & \\
\hline 48 & Pharmacology & $-3.788565 e-01$ & $1.970273 \mathrm{e}-01$ & $5.451898 \mathrm{e}-02$ & \\
\hline 49 & Agriculture & $-1.528354 \mathrm{e}-01$ & $1.592398 \mathrm{e}-01$ & $3.371831 \mathrm{e}-01$ & \\
\hline 50 & Neuroscience & $4.510360 \mathrm{e}-01$ & $1.059519 \mathrm{e}-01$ & $2.086479 \mathrm{e}-05$ & $* * *$ \\
\hline 51 & Fishery & $-3.954242 \mathrm{e}-01$ & $2.267028 \mathrm{e}-01$ & $8.114069 \mathrm{e}-02$ & \\
\hline 52 & Virology & $2.989246 \mathrm{e}-01$ & $1.022268 \mathrm{e}-01$ & $3.460129 \mathrm{e}-03$ & $* *$ \\
\hline 53 & Microbiology & $-2.883729 \mathrm{e}-02$ & $1.520552 \mathrm{e}-01$ & $8.495863 \mathrm{e}-01$ & \\
\hline 54 & Communication & $-2.240787 e-01$ & $1.561387 \mathrm{e}-01$ & $1.512752 \mathrm{e}-01$ & \\
\hline 55 & Endocrinology & $-2.385909 \mathrm{e}-02$ & $5.034799 \mathrm{e}-02$ & $6.355909 \mathrm{e}-01$ & \\
\hline 56 & Epigenetics & $-2.909352 \mathrm{e}-01$ & $1.507975 \mathrm{e}-01$ & $5.371396 \mathrm{e}-02$ & \\
\hline 57 & Marketing & 4.620001e-02 & $1.626733 \mathrm{e}-01$ & $7.764112 \mathrm{e}-01$ & \\
\hline 58 & Global_health & $-3.791645 e-01$ & $2.389615 \mathrm{e}-01$ & $1.126007 \mathrm{e}-01$ & \\
\hline 59 & Agroforestry & $3.715963 \mathrm{e}-01$ & $2.087886 \mathrm{e}-01$ & $7.513637 \mathrm{e}-02$ & \\
\hline 60 & Chemistry & $9.905706 \mathrm{e}-02$ & $1.744052 \mathrm{e}-01$ & $5.700645 \mathrm{e}-01$ & \\
\hline 61 & Mathematics & $8.543681 \mathrm{e}-01$ & $5.082665 \mathrm{e}-01$ & $9.279750 \mathrm{e}-02$ & \\
\hline 62 & Gynecology & $-9.194586 \mathrm{e}-03$ & $8.338434 \mathrm{e}-02$ & $9.121989 \mathrm{e}-01$ & \\
\hline 63 & Gerontology & $1.440034 \mathrm{e}-01$ & $8.837044 \mathrm{e}-02$ & $1.032223 \mathrm{e}-01$ & \\
\hline 64 & Meteorology & $-1.050708 \mathrm{e}-02$ & $2.642057 \mathrm{e}-01$ & $9.682782 \mathrm{e}-01$ & \\
\hline 65 & Veterinary_medicine & $-3.906032 \mathrm{e}-01$ & $6.031535 \mathrm{e}-01$ & $5.172541 \mathrm{e}-01$ & \\
\hline 66 & Government & $-3.794836 \mathrm{e}-02$ & $1.773865 \mathrm{e}-01$ & $8.306047 \mathrm{e}-01$ & \\
\hline
\end{tabular}


Dentistry

Microeconomics

Sociology

Emergency_medicine

Pediatrics

Advertising

Oceanography

Developmental_psychology

Internal_medicine

Cardiology

Environmental_engineering

General_surgery

Environmental_science

Geophysics

Immunology

Clinical_psychology

Toxicology

Ecology

Biology

Epidemiology

Physical_therapy

Physiology

Statistics

Psychopathology

Animal_science

Nursing

Seismology

History

Psychiatry

Zoology

Psychology

Etiology

Geomorphology

Atmospheric_sciences

Geography

Demography

Geology

Medicine

Family_medicine

Evolutionary_biology

Astrobiology

Astronomy

Astrophysics

Geochemistry

Business

Finance

Management_science

Social_psychology

Political_science

Archaeology

Economics

Paleontology
Criminology

\begin{tabular}{|c|c|c|c|}
\hline$-6.139550 \mathrm{e}-01$ & $3.270224 \mathrm{e}-01$ & $6.048427 \mathrm{e}-02$ & \\
\hline$-8.877093 e-02$ & $2.779616 \mathrm{e}-01$ & $7.494556 \mathrm{e}-01$ & \\
\hline $1.694921 \mathrm{e}-02$ & $3.468505 \mathrm{e}-01$ & $9.610268 \mathrm{e}-01$ & \\
\hline $1.935680 \mathrm{e}-03$ & 7.395207e-02 & $9.791183 \mathrm{e}-01$ & \\
\hline $7.633896 \mathrm{e}-02$ & $5.940183 \mathrm{e}-02$ & $1.987703 \mathrm{e}-01$ & \\
\hline $3.952515 \mathrm{e}-02$ & $1.609798 \mathrm{e}-01$ & $8.060510 \mathrm{e}-01$ & \\
\hline$-5.073750 e-01$ & $2.453483 e-01$ & $3.866173 e-02$ & * \\
\hline$-1.730768 \mathrm{e}-01$ & $8.870048 \mathrm{e}-02$ & $5.104864 \mathrm{e}-02$ & \\
\hline $1.950306 \mathrm{e}-01$ & $4.852633 \mathrm{e}-02$ & $5.875713 e-05$ & $* * *$ \\
\hline $1.617686 \mathrm{e}-01$ & $7.721665 e-02$ & $3.619051 \mathrm{e}-02$ & * \\
\hline$-2.655198 \mathrm{e}-02$ & $2.328470 \mathrm{e}-01$ & $9.092143 e-01$ & \\
\hline $1.466085 \mathrm{e}-01$ & $9.005376 \mathrm{e}-02$ & $1.035468 \mathrm{e}-01$ & \\
\hline$-3.470646 e-01$ & $1.834293 \mathrm{e}-01$ & $5.850123 \mathrm{e}-02$ & \\
\hline$-4.657077 \mathrm{e}-01$ & $4.762229 \mathrm{e}-01$ & $3.281322 \mathrm{e}-01$ & \\
\hline $1.767732 \mathrm{e}-02$ & $6.367703 \mathrm{e}-02$ & $7.813169 \mathrm{e}-01$ & \\
\hline$-8.542353 e-02$ & $9.191893 \mathrm{e}-02$ & $3.527324 \mathrm{e}-01$ & \\
\hline $5.989579 \mathrm{e}-01$ & $2.967383 \mathrm{e}-01$ & $4.356253 \mathrm{e}-02$ & * \\
\hline$-1.713938 \mathrm{e}-01$ & $7.081201 \mathrm{e}-02$ & $1.551673 \mathrm{e}-02$ & * \\
\hline$-6.912513 e-04$ & $1.357552 \mathrm{e}-01$ & $9.959374 \mathrm{e}-01$ & \\
\hline$-4.553402 \mathrm{e}-02$ & $7.454185 \mathrm{e}-02$ & $5.413084 \mathrm{e}-01$ & \\
\hline $1.308848 \mathrm{e}-01$ & $4.849343 \mathrm{e}-02$ & $6.963346 \mathrm{e}-03$ & ** \\
\hline $4.251861 \mathrm{e}-01$ & $1.872337 \mathrm{e}-01$ & $2.317023 \mathrm{e}-02$ & * \\
\hline$-3.464209 \mathrm{e}-02$ & $5.993134 \mathrm{e}-01$ & $9.539065 e-01$ & \\
\hline $1.303805 e-02$ & $2.173315 \mathrm{e}-01$ & $9.521633 e-01$ & \\
\hline $6.525120 \mathrm{e}-01$ & $3.395244 \mathrm{e}-01$ & $5.464749 \mathrm{e}-02$ & \\
\hline $5.183049 \mathrm{e}-02$ & $8.807419 \mathrm{e}-02$ & $5.562158 \mathrm{e}-01$ & \\
\hline$-1.004412 \mathrm{e}-01$ & $3.724409 \mathrm{e}-01$ & $7.874080 \mathrm{e}-01$ & \\
\hline $9.556569 \mathrm{e}-01$ & $1.042109 \mathrm{e}+00$ & $3.591378 \mathrm{e}-01$ & \\
\hline $6.389302 \mathrm{e}-02$ & $6.174125 \mathrm{e}-02$ & $3.007575 \mathrm{e}-01$ & \\
\hline$-2.183456 \mathrm{e}-01$ & $1.619297 \mathrm{e}-01$ & $1.775540 \mathrm{e}-01$ & \\
\hline$-1.452703 e-03$ & $1.347180 \mathrm{e}-01$ & $9.913965 \mathrm{e}-01$ & \\
\hline $3.201071 \mathrm{e}-01$ & $1.727141 \mathrm{e}-01$ & $6.384867 \mathrm{e}-02$ & \\
\hline $1.084731 \mathrm{e}-01$ & $2.031706 \mathrm{e}-01$ & $5.934189 \mathrm{e}-01$ & \\
\hline $2.414889 \mathrm{e}-01$ & $2.179970 \mathrm{e}-01$ & $2.679848 \mathrm{e}-01$ & \\
\hline $4.013778 \mathrm{e}-01$ & $1.873654 \mathrm{e}-01$ & $3.219426 \mathrm{e}-02$ & * \\
\hline$-7.158830 \mathrm{e}-02$ & $5.693158 \mathrm{e}-02$ & $2.086152 \mathrm{e}-01$ & \\
\hline$-5.614338 \mathrm{e}-01$ & $2.036943 \mathrm{e}-01$ & $5.854819 \mathrm{e}-03$ & $* *$ \\
\hline $1.566053 \mathrm{e}-01$ & $1.160822 \mathrm{e}-01$ & $1.773316 \mathrm{e}-01$ & \\
\hline$-1.136435 e-01$ & $5.803649 \mathrm{e}-02$ & $5.023495 \mathrm{e}-02$ & \\
\hline$-1.564941 \mathrm{e}-01$ & $1.447539 \mathrm{e}-01$ & $2.796704 \mathrm{e}-01$ & \\
\hline $1.057965 \mathrm{e}+00$ & $3.082093 \mathrm{e}-01$ & $5.996371 \mathrm{e}-04$ & *** \\
\hline$-1.179102 \mathrm{e}-01$ & $2.312251 \mathrm{e}-01$ & $6.101043 \mathrm{e}-01$ & \\
\hline$-1.491296 \mathrm{e}-01$ & $3.566812 \mathrm{e}-01$ & $6.758784 \mathrm{e}-01$ & \\
\hline$-3.557819 \mathrm{e}-01$ & $3.462387 \mathrm{e}-01$ & $3.041748 \mathrm{e}-01$ & \\
\hline$-2.892817 \mathrm{e}-01$ & $1.977081 \mathrm{e}-01$ & $1.434432 \mathrm{e}-01$ & \\
\hline$-3.413238 \mathrm{e}-02$ & $2.094405 \mathrm{e}-01$ & $8.705451 \mathrm{e}-01$ & \\
\hline$-2.236258 \mathrm{e}-12$ & $2.638928 \mathrm{e}-12$ & $3.967813 \mathrm{e}-01$ & \\
\hline $2.732630 \mathrm{e}-02$ & $6.779648 \mathrm{e}-02$ & $6.869080 \mathrm{e}-01$ & \\
\hline $9.537054 \mathrm{e}-02$ & $3.149729 \mathrm{e}-01$ & $7.620550 \mathrm{e}-01$ & \\
\hline$-1.895027 \mathrm{e}-01$ & $3.063191 \mathrm{e}-01$ & $5.361612 \mathrm{e}-01$ & \\
\hline$-1.445690 \mathrm{e}-01$ & $1.547289 \mathrm{e}-01$ & $3.501470 \mathrm{e}-01$ & \\
\hline$-3.775348 \mathrm{e}-01$ & $1.895223 \mathrm{e}-01$ & $4.638818 \mathrm{e}-02$ & $*$ \\
\hline $1.236535 \mathrm{e}+00$ & $4.413599 \mathrm{e}-01$ & $5.091708 \mathrm{e}-03$ & $* *$ \\
\hline
\end{tabular}

Table 6: Regression coefficients for predicting sentence-level certainty with the source of the finding (RQ1). 


\begin{tabular}{|c|c|c|c|c|c|c|}
\hline & DV & IV & coeff & SE & $\mathrm{p}$ value & \\
\hline 1 & Certain_Number & source[T.news] & $-3.754525 e-02$ & $7.430325 \mathrm{e}-03$ & $4.408625 \mathrm{e}-07$ & $* * *$ \\
\hline 3 & Certain_Number & flesch_reading_ease & $4.748661 \mathrm{e}-03$ & $1.672621 \mathrm{e}-04$ & $4.235161 \mathrm{e}-172$ & $* * *$ \\
\hline 4 & Certain_Number & first_author_rank & $1.060523 \mathrm{e}-05$ & $2.141796 \mathrm{e}-06$ & $7.454170 \mathrm{e}-07$ & $* * *$ \\
\hline 5 & Certain_Number & affi_rank & $-1.366679 \mathrm{e}-06$ & $2.136081 \mathrm{e}-06$ & $5.223096 \mathrm{e}-01$ & \\
\hline 7 & Certain_Number & journal_impact & $6.182535 \mathrm{e}-04$ & $3.002580 \mathrm{e}-04$ & $3.950693 \mathrm{e}-02$ & $*$ \\
\hline 8 & Certain_Number & Law & $5.577421 \mathrm{e}-02$ & $1.456364 \mathrm{e}-01$ & $7.017490 \mathrm{e}-01$ & \\
\hline 9 & Certain_Number & Condensed_matter_physics & $-7.395243 \mathrm{e}-02$ & $2.967220 \mathrm{e}-01$ & $8.031858 \mathrm{e}-01$ & \\
\hline 10 & Certain_Number & Biomedical_engineering & $8.751209 \mathrm{e}-01$ & $2.803313 \mathrm{e}-01$ & $1.801792 \mathrm{e}-03$ & $* *$ \\
\hline 11 & Certain_Number & Hydrology & $-4.647589 \mathrm{e}-01$ & $2.458729 \mathrm{e}-01$ & $5.874791 \mathrm{e}-02$ & \\
\hline 16 & Certain_Number & Lung_cancer & $-2.536249 \mathrm{e}-01$ & $6.328336 \mathrm{e}-02$ & $6.163637 \mathrm{e}-05$ & $* * *$ \\
\hline 17 & Certain_Number & Electronic_engineering & $9.203717 \mathrm{e}-01$ & $7.281703 \mathrm{e}-01$ & $2.062701 \mathrm{e}-01$ & \\
\hline 18 & Certain_Number & Artificial_intelligence & $6.991055 \mathrm{e}-01$ & $3.682111 \mathrm{e}-01$ & $5.763200 \mathrm{e}-02$ & \\
\hline 19 & Certain_Number & Machine_learning & $-8.507687 \mathrm{e}-01$ & $3.900474 \mathrm{e}-01$ & $2.918694 \mathrm{e}-02$ & $*$ \\
\hline 20 & Certain_Number & Gender_studies & $-4.482301 \mathrm{e}-01$ & $2.345034 \mathrm{e}-01$ & $5.597490 \mathrm{e}-02$ & \\
\hline 21 & Certain_Number & Chemical_engineering & $-7.470819 \mathrm{e}-01$ & $2.737512 \mathrm{e}-01$ & $6.360124 \mathrm{e}-03$ & $* *$ \\
\hline 22 & Certain_Number & Biophysics & $-3.674674 \mathrm{e}-01$ & $1.679537 \mathrm{e}-01$ & $2.869387 \mathrm{e}-02$ & $*$ \\
\hline 23 & Certain_Number & Analytical_chemistry & $5.311389 \mathrm{e}-01$ & $2.978242 \mathrm{e}-01$ & $7.454493 e-02$ & \\
\hline 24 & Certain_Number & Computer_science & $-1.515536 \mathrm{e}-02$ & $1.961629 \mathrm{e}-01$ & $9.384186 \mathrm{e}-01$ & \\
\hline 25 & Certain_Number & Radiology & $1.473517 \mathrm{e}-01$ & $6.990715 \mathrm{e}-02$ & $3.506558 \mathrm{e}-02$ & $*$ \\
\hline 26 & Certain_Number & Urology & $4.019890 \mathrm{e}-01$ & $1.056941 \mathrm{e}-01$ & $1.434167 \mathrm{e}-04$ & $* * *$ \\
\hline 34 & Certain_Number & Molecular_biology & $-1.017168 \mathrm{e}-01$ & $6.981345 \mathrm{e}-02$ & $1.451460 \mathrm{e}-01$ & \\
\hline 35 & Certain_Number & Dermatology & $2.521797 \mathrm{e}-01$ & $9.509705 \mathrm{e}-02$ & $8.015723 \mathrm{e}-03$ & $* *$ \\
\hline 36 & Certain_Number & Engineering & $7.041088 \mathrm{e}-01$ & $2.531793 \mathrm{e}-01$ & $5.425810 \mathrm{e}-03$ & $* *$ \\
\hline 37 & Certain_Number & Surgery & $2.247965 \mathrm{e}-01$ & $3.550478 \mathrm{e}-02$ & $2.508371 \mathrm{e}-10$ & $* * *$ \\
\hline 38 & Certain_Number & Public_health & $-2.287177 \mathrm{e}-01$ & $4.838632 \mathrm{e}-02$ & $2.303450 \mathrm{e}-06$ & $* * *$ \\
\hline 39 & Certain_Number & Molecular_physics & $1.390593 \mathrm{e}+00$ & $3.492814 \mathrm{e}-01$ & $6.891166 \mathrm{e}-05$ & $* * *$ \\
\hline 40 & Certain_Number & Bioinformatics & $9.525464 \mathrm{e}-02$ & $1.600507 \mathrm{e}-01$ & $5.517516 \mathrm{e}-01$ & \\
\hline 41 & Certain_Number & Physics & $-1.645778 \mathrm{e}-01$ & $1.817700 \mathrm{e}-01$ & $3.652609 \mathrm{e}-01$ & \\
\hline 42 & Certain_Number & Cognitive_psychology & $-1.615658 \mathrm{e}-01$ & $9.113908 \mathrm{e}-02$ & $7.629535 \mathrm{e}-02$ & \\
\hline 43 & Certain_Number & Oncology & $3.425175 \mathrm{e}-01$ & $6.270263 \mathrm{e}-02$ & $4.778901 \mathrm{e}-08$ & $* * *$ \\
\hline 44 & Certain_Number & Pathology & $-6.613642 \mathrm{e}-02$ & $6.503292 \mathrm{e}-02$ & $3.091873 \mathrm{e}-01$ & \\
\hline 45 & Certain_Number & Biochemistry & $6.366612 \mathrm{e}-02$ & $8.905872 \mathrm{e}-02$ & $4.746972 \mathrm{e}-01$ & \\
\hline 46 & Certain_Number & Social_science & $2.521395 \mathrm{e}-02$ & $3.544800 \mathrm{e}-01$ & $9.432959 \mathrm{e}-01$ & \\
\hline 47 & Certain_Number & Climatology & $1.761927 \mathrm{e}-01$ & $9.846454 \mathrm{e}-02$ & $7.357319 \mathrm{e}-02$ & \\
\hline 48 & Certain_Number & Pharmacology & $-4.634962 \mathrm{e}-01$ & $1.421333 \mathrm{e}-01$ & $1.113058 \mathrm{e}-03$ & $* *$ \\
\hline 49 & Certain_Number & Agriculture & $1.785342 \mathrm{e}-01$ & $1.148738 \mathrm{e}-01$ & $1.201666 \mathrm{e}-01$ & \\
\hline 50 & Certain_Number & Neuroscience & $-2.605759 \mathrm{e}-01$ & $7.643253 \mathrm{e}-02$ & $6.534419 \mathrm{e}-04$ & $* * *$ \\
\hline 51 & Certain_Number & Fishery & $3.498395 \mathrm{e}-01$ & $1.635409 \mathrm{e}-01$ & $3.244152 \mathrm{e}-02$ & $*$ \\
\hline 52 & Certain_Number & Virology & $-2.766294 \mathrm{e}-01$ & $7.374527 \mathrm{e}-02$ & $1.767884 \mathrm{e}-04$ & $* * *$ \\
\hline 53 & Certain_Number & Microbiology & $2.659344 \mathrm{e}-01$ & $1.096909 \mathrm{e}-01$ & $1.534729 \mathrm{e}-02$ & $*$ \\
\hline
\end{tabular}




\begin{tabular}{|c|c|}
\hline 66 & \\
\hline 67 & Certain_Number \\
\hline & Certain_Number \\
\hline & Certain_Number \\
\hline & \\
\hline & \\
\hline & \\
\hline & Certain_I \\
\hline & Certain_Nur \\
\hline & Certain_Nur \\
\hline & Certain_Nu \\
\hline & Certain_Nuı \\
\hline & Certain_Nun \\
\hline & Certain_Nur \\
\hline & Certain_Nur \\
\hline & Certain_N \\
\hline & \\
\hline & \\
\hline & \\
\hline & \\
\hline & \\
\hline & \\
\hline & Cer \\
\hline & Cer \\
\hline 90 & Cer \\
\hline & Cert \\
\hline & Cert \\
\hline & Cer \\
\hline & Cer \\
\hline & \\
\hline & \\
\hline & \\
\hline & \\
\hline & \\
\hline & \\
\hline & \\
\hline & \\
\hline & \\
\hline & \\
\hline & Cer \\
\hline & Cer \\
\hline & Cer \\
\hline & \\
\hline & \\
\hline & \\
\hline & \\
\hline & \\
\hline & \\
\hline & \\
\hline & Cer \\
\hline & Cer \\
\hline & Cer \\
\hline & Cert \\
\hline & Cert \\
\hline & Cert \\
\hline & Cert \\
\hline & Cert \\
\hline & Certain_Ext \\
\hline & Certain_Extent \\
\hline & Certain_Ext \\
\hline & Cert \\
\hline & ert \\
\hline & \\
\hline & Certa \\
\hline & Certain_Extent \\
\hline & Certain_Extent \\
\hline & \\
\hline & \\
\hline & \\
\hline & Certain_Exte \\
\hline
\end{tabular}

Government

Dentistry

Microeconomics

Sociology

Emergency_medicine

Pediatrics

Advertising

Oceanography

Developmental_psychology

Internal_medicine

Cardiology

Environmental_engineering

General_surgery

Environmental_science

Geophysics

Immunology

Clinical_psychology

Toxicology

Ecology

Biology

Epidemiology

Physical_therapy

Physiology

Statistics

Psychopathology

Animal_science

Nursing

Seismology

History

Psychiatry

Zoology

Psychology

Etiology

Geomorphology

Atmospheric_sciences

Geography

Demography

Geology

Medicine

Family_medicine

Evolutionary_biology

Astrobiology

Astronomy

Astrophysics

Geochemistry

Business

Finance

Management_science

Social_psychology

Political_science

Archaeology

Economics

Paleontology

Criminology

Intercept

source[T.news]

finding_len

flesch_reading_ease

first_author_rank

affi_rank

num_authors

journal_impact

Law

Condensed_matter_physics

Biomedical_engineering

Hydrology

Atomic_physics

Development_economics

Optics

Public_policy
$-7.773482 \mathrm{e}-02$

$1.507756 \mathrm{e}-01$

$2.584703 \mathrm{e}-05$

$1.430314 \mathrm{e}-01$

$2.686383 \mathrm{e}-01$

$1.175814 \mathrm{e}-01$

$3.670273 \mathrm{e}-01$

$3.029326 \mathrm{e}-03$

$5.964269 \mathrm{e}-02$

2.653652e- 01

$-6.553795 \mathrm{e}-02$

$2.538147 \mathrm{e}-01$

$1.462771 \mathrm{e}-01$

$-2.904045 \mathrm{e}-02$

$-2.955363 \mathrm{e}-01$

$-1.782613 \mathrm{e}-01$

$-3.273513 \mathrm{e}-02$

$5.669777 \mathrm{e}-01$

$1.850180 \mathrm{e}-01$

$-1.320305 \mathrm{e}-01$

$-1.000484 \mathrm{e}-01$

$7.809445 \mathrm{e}-02$

$-2.939125 \mathrm{e}-01$

$-5.536512 \mathrm{e}-01$

$-2.857243 \mathrm{e}-01$

$-7.908161 \mathrm{e}-02$

$1.563805 \mathrm{e}-01$

$2.995495 \mathrm{e}-01$

$2.592724 \mathrm{e}+00$

$-5.759278 \mathrm{e}-03$

$-8.188208 \mathrm{e}-02$

$3.233777 \mathrm{e}-01$

2.373062e-02

$-2.879758 \mathrm{e}-01$

$-3.494302 \mathrm{e}-01$

1.106072e-01

$1.177766 \mathrm{e}-01$

$-2.362485 \mathrm{e}-01$

$6.047733 \mathrm{e}-01$

$1.612269 \mathrm{e}-02$

$-2.159382 \mathrm{e}-01$

$-1.207153 \mathrm{e}-02$

$-3.409237 \mathrm{e}-01$

$-9.119224 \mathrm{e}-02$

$-4.338617 \mathrm{e}-01$

$1.070303 \mathrm{e}-01$

$3.223504 \mathrm{e}-01$

$-8.307473 \mathrm{e}-12$

$-3.996282 \mathrm{e}-01$

$-1.130388 \mathrm{e}-02$

$-6.032563 \mathrm{e}-01$

$-1.040828 \mathrm{e}-01$

$-3.794069 \mathrm{e}-02$

$-6.505262 \mathrm{e}-02$

$-3.725410 \mathrm{e}-01$

$3.123037 \mathrm{e}-03$

$9.380603 \mathrm{e}-03$

$2.641308 \mathrm{e}-03$

$1.392175 \mathrm{e}-05$

$-2.632459 \mathrm{e}-06$

$1.326017 \mathrm{e}-03$

$1.971570 \mathrm{e}-04$

$1.365785 \mathrm{e}-01$

$-3.726838 \mathrm{e}-01$

$-3.047912 \mathrm{e}-01$

$-3.089827 \mathrm{e}-01$

$-3.859061 \mathrm{e}-01$

$-6.149000 \mathrm{e}-02$

$-3.448521 \mathrm{e}-01$

$1.278272 \mathrm{e}-01$
$1.279646 \mathrm{e}-01$

$2.359103 \mathrm{e}-01$

$2.005184 \mathrm{e}-01$

$2.502141 \mathrm{e}-01$

$5.334820 \mathrm{e}-02$

4.285181e-02

$1.161290 \mathrm{e}-01$

$1.769915 \mathrm{e}-01$

$6.398754 \mathrm{e}-02$

$3.500635 \mathrm{e}-02$

$5.570323 \mathrm{e}-02$

$1.679733 \mathrm{e}-01$

$6.496378 \mathrm{e}-02$

$1.323239 \mathrm{e}-01$

$3.435419 \mathrm{e}-01$

$4.593590 \mathrm{e}-02$

$6.630929 \mathrm{e}-02$

$2.140637 \mathrm{e}-01$

$5.108299 \mathrm{e}-02$

$9.793229 \mathrm{e}-02$

$5.377365 \mathrm{e}-02$

$3.498262 \mathrm{e}-02$

$1.350683 \mathrm{e}-01$

$4.323380 \mathrm{e}-01$

$1.567805 \mathrm{e}-01$

2.449291e-01

$6.353573 \mathrm{e}-02$

$2.686747 \mathrm{e}-01$

$7.517655 \mathrm{e}-01$

$4.453945 \mathrm{e}-02$

$1.168143 \mathrm{e}-01$

9.718405e-02

$1.245940 \mathrm{e}-01$

$1.465650 \mathrm{e}-01$

$1.572606 \mathrm{e}-01$

$1.351633 \mathrm{e}-01$

4.106981e-02

$1.469428 \mathrm{e}-01$

$8.374042 \mathrm{e}-02$

4.186687e-02

$1.044238 \mathrm{e}-01$

$2.223387 \mathrm{e}-01$

$1.668032 \mathrm{e}-01$

$2.573058 \mathrm{e}-01$

$2.497727 \mathrm{e}-01$

$1.426244 \mathrm{e}-01$

$1.510880 \mathrm{e}-01$

$2.422168 \mathrm{e}-12$

4.890762e-02

$2.272179 \mathrm{e}-01$

$2.209751 \mathrm{e}-01$

$1.116197 \mathrm{e}-01$

$1.367193 \mathrm{e}-01$

$3.183921 \mathrm{e}-01$

$5.367789 \mathrm{e}-02$

7.541623e-03

$4.256270 \mathrm{e}-04$

$1.697675 \mathrm{e}-04$

$2.173877 \mathrm{e}-06$

$2.168077 \mathrm{e}-06$

$2.955833 \mathrm{e}-04$

$3.047555 \mathrm{e}-04$

$1.478179 \mathrm{e}-01$

3.011666e-01

$2.845303 \mathrm{e}-01$

$2.495557 \mathrm{e}-01$

$3.542185 \mathrm{e}-01$

$4.030912 \mathrm{e}-01$

$4.397638 \mathrm{e}-01$

2.705637e-01
$5.435488 \mathrm{e}-01$

$5.227544 \mathrm{e}-01$

$9.998972 \mathrm{e}-01$

$5.675783 e-01$

$4.827989 \mathrm{e}-07$

$6.079575 \mathrm{e}-03$

$1.578516 \mathrm{e}-03$

9.863446e-01

$3.513028 \mathrm{e}-01$

$3.674670 \mathrm{e}-14$

$2.393943 \mathrm{e}-01$

$1.308018 \mathrm{e}-01$

$2.435957 \mathrm{e}-02$

$8.262913 \mathrm{e}-01$

$3.896600 \mathrm{e}-01$

$1.046880 \mathrm{e}-04$

6.215451e-01

$8.091347 \mathrm{e}-03$

$2.935440 \mathrm{e}-04$

$1.776233 \mathrm{e}-01$

$6.283068 \mathrm{e}-02$

$2.560685 \mathrm{e}-02$

$2.957104 \mathrm{e}-02$

$2.003576 \mathrm{e}-01$

$6.840990 \mathrm{e}-02$

7.467947e-01

$1.385631 \mathrm{e}-02$

$2.649073 \mathrm{e}-01$

5.647643e-04

8.971164e-01

$4.833408 \mathrm{e}-01$

8.787826e-04

$8.489489 \mathrm{e}-01$

$4.945470 \mathrm{e}-02$

$2.630164 \mathrm{e}-02$

4.131878e-01

$4.141060 \mathrm{e}-03$

$1.079123 \mathrm{e}-01$

$5.406783 \mathrm{e}-13$

$7.001738 \mathrm{e}-01$

$3.866894 \mathrm{e}-02$

$9.567022 \mathrm{e}-01$

$4.098661 \mathrm{e}-02$

$7.230359 \mathrm{e}-01$

$8.240618 \mathrm{e}-02$

$4.530064 \mathrm{e}-01$

$3.290007 \mathrm{e}-02$

6.059682e-04

$3.336214 \mathrm{e}-16$

9.603231e-01

$6.342432 \mathrm{e}-03$

$3.511073 \mathrm{e}-01$

$7.813945 \mathrm{e}-01$

$8.381097 \mathrm{e}-01$

$4.097312 \mathrm{e}-12$

$6.788027 \mathrm{e}-01$

$1.009276 \mathrm{e}-105$

$4.268109 \mathrm{e}-54$

$1.564327 \mathrm{e}-10$

$2.246969 \mathrm{e}-01$

$7.315738 \mathrm{e}-06$

$5.176855 \mathrm{e}-01$

3.555217e-01

$2.159359 \mathrm{e}-01$

$2.840956 \mathrm{e}-01$

$2.156897 \mathrm{e}-01$

2.759721e-01

8.787586e-01

4.329513e-01

$6.366151 \mathrm{e}-01$

$* * *$

$* *$

$* *$

$* * *$

$*$

$* * *$

$* *$ 


\begin{tabular}{|c|c|c|}
\hline 136 & Certain_Extent & Lung_cancer \\
\hline 137 & Certain_Extent & Electronic_engineering \\
\hline 138 & Certain_Extent & Artificial_intelligence \\
\hline 139 & Certain_Extent & Machine_learning \\
\hline 140 & Certain_Extent & Gender_studies \\
\hline 141 & Certain_Extent & Chemical_engineering \\
\hline 142 & Certain_Extent & Biophysics \\
\hline 143 & Certain_Extent & Analytical_chemistry \\
\hline 144 & Certain_Extent & Computer_science \\
\hline 145 & Certain_Extent & Radiology \\
\hline 146 & Certain_Extent & Urology \\
\hline 147 & Certain_Extent & Architecture \\
\hline 148 & Certain_Extent & Quantum_mechanics \\
\hline 149 & Certain_Extent & Particle_physics \\
\hline 150 & Certain_Extent & Materials_science \\
\hline 151 & Certain_Extent & Computational_biology \\
\hline 152 & Certain_Extent & Agronomy \\
\hline 153 & Certain_Extent & $\begin{array}{l}\text { Environmental_resource man- } \\
\text { agement }\end{array}$ \\
\hline 154 & Certain_Extent & Molecular_biology \\
\hline 155 & Certain_Extent & Dermatology \\
\hline 156 & Certain_Extent & Engineering \\
\hline 157 & Certain_Extent & Surgery \\
\hline 158 & Certain_Extent & Public_health \\
\hline 159 & Certain_Extent & Molecular_physics \\
\hline 160 & Certain_Extent & Bioinformatics \\
\hline 161 & Certain_Extent & Physics \\
\hline 162 & Certain_Extent & Cognitive_psychology \\
\hline 163 & Certain_Extent & Oncology \\
\hline 164 & Certain_Extent & Pathology \\
\hline 165 & Certain_Extent & Biochemistry \\
\hline 166 & Certain_Extent & Social_science \\
\hline 167 & Certain_Extent & Climatology \\
\hline 168 & Certain_Extent & Pharmacology \\
\hline 169 & Certain_Extent & Agriculture \\
\hline 170 & Certain_Extent & Neuroscience \\
\hline 171 & Certain_Extent & Fishery \\
\hline 172 & Certain_Extent & Virology \\
\hline 173 & Certain_Extent & Microbiology \\
\hline 174 & Certain_Extent & Communication \\
\hline 175 & Certain_Extent & Endocrinology \\
\hline 176 & Certain_Extent & Epigenetics \\
\hline 177 & Certain_Extent & Marketing \\
\hline 178 & Certain_Extent & Global_health \\
\hline 179 & Certain_Extent & Agroforestry \\
\hline 180 & Certain_Extent & Chemistry \\
\hline 181 & Certain_Extent & Mathematics \\
\hline 182 & Certain_Extent & Gynecology \\
\hline 183 & Certain_Extent & Gerontology \\
\hline 184 & Certain_Extent & Meteorology \\
\hline 185 & Certain_Extent & Veterinary_medicine \\
\hline 186 & Certain_Extent & Government \\
\hline 187 & Certain_Extent & Dentistry \\
\hline 188 & Certain_Extent & Microeconomics \\
\hline 189 & Certain_Extent & Sociology \\
\hline 190 & Certain_Extent & Emergency_medicine \\
\hline 191 & Certain_Extent & Pediatrics \\
\hline 192 & Certain_Extent & Advertising \\
\hline 193 & Certain_Extent & Oceanography \\
\hline 194 & Certain_Extent & Developmental_psychology \\
\hline 195 & Certain_Extent & Internal_medicine \\
\hline 196 & Certain_Extent & Cardiology \\
\hline 197 & Certain_Extent & Environmental_engineering \\
\hline 198 & Certain_Extent & General_surgery \\
\hline 199 & Certain_Extent & Environmental_science \\
\hline 200 & Certain_Extent & Geophysics \\
\hline 201 & Certain_Extent & Immunology \\
\hline 202 & Certain_Extent & Clinical_psychology \\
\hline 203 & Certain_Extent & Toxicology \\
\hline 204 & Certain_Extent & Ecology \\
\hline
\end{tabular}

$-2.517175 \mathrm{e}-01$
$2.202177 \mathrm{e}+00$
$-4.519185 \mathrm{e}-02$
$-5.946431 \mathrm{e}-01$
$1.206807 \mathrm{e}-01$
$-5.676854 \mathrm{e}-01$
$-3.503262 \mathrm{e}-01$
$5.275629 \mathrm{e}-01$
$2.985267 \mathrm{e}-01$
$3.263423 \mathrm{e}-02$
$1.113795 \mathrm{e}-01$
$-1.576639 \mathrm{e}-01$
$-1.508489 \mathrm{e}+00$
$1.019965 \mathrm{e}+00$
$-1.670032 \mathrm{e}-02$
$-7.717124 \mathrm{e}-02$
$1.629951 \mathrm{e}-02$
$5.206592 \mathrm{e}-01$

$-1.414140 \mathrm{e}-01$

$-1.059578 \mathrm{e}-01$

$6.541285 \mathrm{e}-01$

$1.853908 \mathrm{e}-01$

$-1.145067 \mathrm{e}-01$

$-3.665798 \mathrm{e}-01$

4.947193e-01

9.801933e-02

$-4.366194 \mathrm{e}-01$

$2.287968 \mathrm{e}-01$

$-9.240980 \mathrm{e}-02$

$-1.649307 \mathrm{e}-01$

5.808280e-01

$4.158299 \mathrm{e}-02$

$-3.630674 \mathrm{e}-01$

$-2.610968 \mathrm{e}-01$

$-3.724170 \mathrm{e}-01$

$-1.058346 \mathrm{e}-01$

$-9.194626 \mathrm{e}-02$

$1.047860 \mathrm{e}-01$

$-4.888373 \mathrm{e}-01$

$-8.575610 \mathrm{e}-02$

$-2.715432 \mathrm{e}-01$

$-2.134733 \mathrm{e}-01$

$-9.930930 \mathrm{e}-02$

$3.078368 \mathrm{e}-01$

$-6.882521 \mathrm{e}-02$

$4.593218 \mathrm{e}-01$

$-2.050583 \mathrm{e}-01$

$-1.939865 \mathrm{e}-01$

$2.738084 \mathrm{e}-01$

$-3.434918 \mathrm{e}-01$

4.443504e-01

$-3.602740 \mathrm{e}-01$

2.242987e-01

$2.229463 \mathrm{e}-01$

$1.153556 \mathrm{e}-01$

$1.970765 \mathrm{e}-02$

$2.553217 \mathrm{e}-01$

$-2.076142 \mathrm{e}-01$

$-1.242801 \mathrm{e}-01$

$2.739139 \mathrm{e}-01$

$-4.090315 \mathrm{e}-02$

$-1.736697 \mathrm{e}-01$

6.937721e-02

$2.023994 \mathrm{e}-01$

$-5.916370 \mathrm{e}-02$

$-1.857359 \mathrm{e}-01$

$2.190159 \mathrm{e}-01$

7.894684e-02

$1.343534 \mathrm{e}-01$ 6.423128e-02

$7.390775 \mathrm{e}-01$

$3.737265 \mathrm{e}-01$

$3.958899 \mathrm{e}-01$

$2.380160 \mathrm{e}-01$

$2.778516 \mathrm{e}-01$

$1.704694 \mathrm{e}-01$

$3.022853 \mathrm{e}-01$

$1.991012 \mathrm{e}-01$

$7.095428 \mathrm{e}-02$

$1.072772 \mathrm{e}-01$

$2.435682 \mathrm{e}-01$

9.647197e-01

$8.348254 \mathrm{e}-01$

$1.438900 \mathrm{e}-01$

$3.542475 \mathrm{e}-01$

$1.896609 \mathrm{e}-01$

$1.094026 \mathrm{e}-01$

7.085917e-02

$9.652149 \mathrm{e}-02$

$2.569716 \mathrm{e}-01$

3.603660e-02

$4.911109 \mathrm{e}-02$

$3.545133 \mathrm{e}-01$

$1.624481 \mathrm{e}-01$

$1.844927 \mathrm{e}-01$

$9.250424 \mathrm{e}-02$

$6.364184 \mathrm{e}-02$

$6.600704 \mathrm{e}-02$

9.039272e-02

$3.597897 \mathrm{e}-01$

$9.993942 \mathrm{e}-02$

$1.442623 \mathrm{e}-01$

$1.165945 \mathrm{e}-01$

$7.757740 \mathrm{e}-02$

$1.659905 \mathrm{e}-01$

$7.484989 \mathrm{e}-02$

$1.113340 \mathrm{e}-01$

$1.143239 \mathrm{e}-01$

3.686451e-02

$1.104131 \mathrm{e}-01$

$1.191085 \mathrm{e}-01$

$1.749662 \mathrm{e}-01$

$1.528738 \mathrm{e}-01$

$1.276985 \mathrm{e}-01$

$3.721499 \mathrm{e}-01$

$6.105354 \mathrm{e}-02$

$6.470434 \mathrm{e}-02$

$1.934499 \mathrm{e}-01$

$4.416256 \mathrm{e}-01$

$1.298814 \mathrm{e}-01$

$2.394440 \mathrm{e}-01$

$2.035219 \mathrm{e}-01$

$2.539620 \mathrm{e}-01$

$5.414729 \mathrm{e}-02$

$4.349368 \mathrm{e}-02$

$1.178685 \mathrm{e}-01$

$1.796427 \mathrm{e}-01$

$6.494599 \mathrm{e}-02$

$3.553071 \mathrm{e}-02$

$5.653760 \mathrm{e}-02$

$1.704893 \mathrm{e}-01$

$6.593686 \mathrm{e}-02$

$1.343059 \mathrm{e}-01$

$3.486877 \mathrm{e}-01$

4.662396e-02

$6.730252 \mathrm{e}-02$

2.172701e-01

5.184816e-02
$8.939909 \mathrm{e}-05$

$2.891287 \mathrm{e}-03$

$9.037545 \mathrm{e}-01$

$1.331105 \mathrm{e}-01$

$6.121439 \mathrm{e}-01$

$4.106019 \mathrm{e}-02$

3.989233e-02

$8.096520 \mathrm{e}-02$

$1.338014 \mathrm{e}-01$

$6.455718 \mathrm{e}-01$

2.991776e-01

$5.174434 \mathrm{e}-01$

$1.179228 \mathrm{e}-01$

$2.218164 \mathrm{e}-01$

9.076043e-01

$8.275529 \mathrm{e}-01$

$9.315152 \mathrm{e}-01$

$1.965323 \mathrm{e}-06 \quad * * *$

4.598687e-02

$2.723280 \mathrm{e}-01$

$1.092266 \mathrm{e}-02$

$2.721023 \mathrm{e}-07$

$1.973763 \mathrm{e}-02$

$3.011381 \mathrm{e}-01$

$2.328352 \mathrm{e}-03$

5.952261e-01

$2.383016 \mathrm{e}-06$

$3.255019 \mathrm{e}-04$

$1.615373 \mathrm{e}-01$

$6.808398 \mathrm{e}-02$

$1.064748 \mathrm{e}-01$

$6.773569 \mathrm{e}-01$

$1.185724 \mathrm{e}-02$

$2.514940 \mathrm{e}-02$

$1.599561 \mathrm{e}-06$

$5.237489 \mathrm{e}-01$

$2.193158 \mathrm{e}-01$

3.466267e-01

$1.917212 \mathrm{e}-05$

$2.002042 \mathrm{e}-02$

$1.393218 \mathrm{e}-02$

$7.311454 \mathrm{e}-02$

$5.703224 \mathrm{e}-01$

4.406513e-02

$5.899192 \mathrm{e}-01$

$2.171362 \mathrm{e}-01$

$7.854449 \mathrm{e}-04$

$2.722300 \mathrm{e}-03$

$1.569760 \mathrm{e}-01$

$4.367072 \mathrm{e}-01$

$6.253600 \mathrm{e}-04$

$1.324441 \mathrm{e}-01$

$2.704445 \mathrm{e}-01$

$3.800291 \mathrm{e}-01$

$3.315694 \mathrm{e}-02$

$6.504733 \mathrm{e}-01$

3.031727e-02

$2.478225 \mathrm{e}-01$

$5.569417 \mathrm{e}-02$

$1.357309 \mathrm{e}-14$

$4.694053 \mathrm{e}-01$

3.083860e-01

$2.927381 \mathrm{e}-01$

$1.318342 \mathrm{e}-01$

8.652681e-01

6.821848e-05

$1.140016 \mathrm{e}-03$

7.163433e-01

9.572443e-03
$* * *$

$* *$

$*$

$*$

$*$

$* * *$

$*$

$* *$

$* * *$

$* * *$

***

*

$*$

$*$

$* * *$

$* *$

$* * *$

*

$*$

$* * *$

$* * *$

$* *$

$* *$ 
Certain_Extent

Certain_Extent

Certain_Extent

Certain_Extent

Certain_Extent

Certain_Extent

Certain_Extent

Certain_Extent

Certain_Extent

Certain_Extent

Certain_Extent

Certain_Extent

Certain_Extent

Certain_Extent

Certain_Extent

Certain_Extent

Certain_Extent

Certain_Extent

Certain_Extent

Certain_Extent

Certain_Extent

Certain_Extent

Certain Extent

Certain_Extent

Certain_Extent

Certain_Extent

Certain_Extent

Certain_Extent

Certain_Extent

Certain_Extent

Certain_Extent

Certain_Extent

Certain_Extent

Certain_Extent

Certain_Extent

Certain_Probability

Certain_Probability

Certain_Probability

Certain_Probability

Certain_Probability

Certain_Probability

Certain_Probability

Certain Probability

Certain_Probability

Certain_Probability

Certain_Probability

Certain_Probability

Certain_Probability

Certain_Probability

Certain_Probability

Certain_Probability

Certain_Probability

Certain_Probability

Certain_Probability

Certain_Probability

Certain_Probability

Certain_Probability

Certain_Probability

Certain_Probability

Certain_Probability

Certain_Probability

Certain_Probability

Certain_Probability

Certain_Probability

Certain_Probability

Certain_Probability

Certain_Probability

Certain_Probability

Certain_Probability
Biology

Epidemiology

Physical_therapy

Physiology

Statistics

Psychopathology

Animal_science

Nursing

Seismology

History

Psychiatry

Zoology

Psychology

Etiology

Geomorphology

Atmospheric_sciences

Geography

Demography

Geology

Medicine

Family_medicine

Evolutionary_biology

Astrobiology

Astronomy

Astrophysics

Geochemistry

Business

Finance

Management science

Social_psychology

Political science

Archaeology

Economics

Paleontology

Criminology

Intercept

source[T.news]

finding_len

flesch_reading_ease

first_author_rank

affi_rank

num_authors

journal_impact

Law

Condensed_matter_physics

Biomedical_engineering

Hydrology

Atomic_physics

Development_economics

Optics

Public_policy

Lung_cancer

Electronic_engineering

Artificial_intelligence

Machine_learning

Gender_studies

Chemical_engineering

Biophysics

Analytical_chemistry

Computer_science

Radiology

Urology

Architecture

Quantum_mechanics

Particle_physics

Materials_science

Computational_biology

Agronomy

Environmental_resource management
$-2.735608 \mathrm{e}-02$

$-3.071022 \mathrm{e}-03$

$4.883960 \mathrm{e}-02$

$-1.981651 \mathrm{e}-01$

$-6.162491 \mathrm{e}-01$

$-1.146569 \mathrm{e}-01$

$5.840241 \mathrm{e}-02$

$1.867469 \mathrm{e}-01$

$1.731103 \mathrm{e}-01$

$2.654922 \mathrm{e}+00$

$1.513048 \mathrm{e}-01$

$-1.047777 \mathrm{e}-01$

$4.528562 \mathrm{e}-01$

$-1.697349 \mathrm{e}-01$

$-1.354648 \mathrm{e}-01$

$-2.076946 \mathrm{e}-01$

$8.390256 \mathrm{e}-02$

1.390486e-01

$-7.713766 \mathrm{e}-02$

$7.589976 \mathrm{e}-01$

5.400884e-02

$-1.674566 \mathrm{e}-01$

$-4.670557 \mathrm{e}-02$

$-1.627684 \mathrm{e}-01$

$-2.081467 \mathrm{e}-01$

$-3.457197 \mathrm{e}-01$

$2.450527 \mathrm{e}-01$

$1.139189 \mathrm{e}-01$

$5.511531 \mathrm{e}-13$

$-3.012274 \mathrm{e}-01$

$2.370012 \mathrm{e}-01$

$-2.017902 \mathrm{e}-01$

$1.377498 \mathrm{e}-02$

$-5.052782 \mathrm{e}-02$

$3.196030 \mathrm{e}-01$

$1.057673 \mathrm{e}+00$

$1.140286 \mathrm{e}-02$

$-3.860967 \mathrm{e}-04$

$-2.132858 \mathrm{e}-03$

$-5.539980 \mathrm{e}-06$

$-1.830132 \mathrm{e}-06$

$-1.020527 \mathrm{e}-03$

$-1.165746 \mathrm{e}-04$

$-5.092831 \mathrm{e}-01$

$4.918260 \mathrm{e}-02$

$1.025878 \mathrm{e}-01$

$2.642196 \mathrm{e}-01$

$3.699017 \mathrm{e}-01$

$-3.480610 \mathrm{e}-01$

$-2.425676 \mathrm{e}-01$

$5.261578 \mathrm{e}-01$

$-3.718721 \mathrm{e}-02$

$-1.018596 \mathrm{e}+00$

$-9.673953 \mathrm{e}-03$

$-1.233860 \mathrm{e}+00$

$-6.981121 \mathrm{e}-02$

$3.574786 \mathrm{e}-01$

$7.002946 \mathrm{e}-02$

$-1.352697 \mathrm{e}-02$

$-1.124246 \mathrm{e}-01$

$-3.494218 \mathrm{e}-01$

$2.756886 \mathrm{e}-01$

9.967873e-02

$2.234456 \mathrm{e}-01$

$-8.270649 \mathrm{e}-01$

$8.252067 \mathrm{e}-02$

9.112046e-02

$-6.708054 \mathrm{e}-01$

$-3.221610 \mathrm{e}-01$

$9.939920 \mathrm{e}-02$

$5.457912 \mathrm{e}-02$

$3.550662 \mathrm{e}-02$

$1.370915 \mathrm{e}-01$

$4.388139 \mathrm{e}-01$

$1.591289 \mathrm{e}-01$

$2.485979 \mathrm{e}-01$

$6.448742 \mathrm{e}-02$

$2.726992 \mathrm{e}-01$

7.630261e-01

$4.520660 \mathrm{e}-02$

$1.185640 \mathrm{e}-01$

$9.863976 \mathrm{e}-02$ 
Certain_Probability Certain_Probability Certain_Probability Certain_Probability Certain_Probability Certain_Probability Certain_Probability Certain_Probability Certain_Probability Certain_Probability Certain_Probability Certain_Probability Certain_Probability Certain_Probability Certain_Probability Certain_Probability Certain_Probability Certain_Probability Certain_Probability Certain_Probability Certain_Probability Certain_Probability Certain_Probability Certain_Probability Certain_Probability Certain_Probability Certain_Probability Certain_Probability Certain_Probability Certain_Probability Certain_Probability Certain_Probability Certain_Probability Certain_Probability Certain_Probability Certain_Probability Certain_Probability Certain_Probability Certain_Probability Certain_Probability Certain_Probability Certain_Probability Certain_Probability Certain_Probability Certain_Probability Certain_Probability Certain_Probability Certain_Probability Certain_Probability Certain_Probability Certain_Probability Certain_Probability Certain_Probability Certain_Probability Certain_Probability Certain_Probability Certain_Probability Certain_Probability Certain_Probability Certain_Probability Certain_Probability Certain_Probability Certain_Probability Certain_Probability Certain_Probability Certain_Probability Certain_Probability Certain_Probability Certain_Probability Certain_Probability
Molecular_biology

Dermatology

Engineering

Surgery

Public_health

Molecular_physics

Bioinformatics

Physics

Cognitive_psychology

Oncology

Pathology

Biochemistry

Social_science

Climatology

Pharmacology

Agriculture

Neuroscience

Fishery

Virology

Microbiology

Communication

Endocrinology

Epigenetics

Marketing

Global_health

Agroforestry

Chemistry

Mathematics

Gynecology

Gerontology

Meteorology

Veterinary_medicine

Government

Dentistry

Microeconomics

Sociology

Emergency_medicine

Pediatrics

Advertising

Oceanography

Developmental_psychology

Internal_medicine

Cardiology

Environmental_engineering

General_surgery

Environmental_science

Geophysics

Immunology

Clinical_psychology

Toxicology

Ecology

Biology

Epidemiology

Physical_therapy

Physiology

Statistics

Psychopathology

Animal_science

Nursing

Seismology

History

Psychiatry

Zoology

Psychology

Etiology

Geomorphology

Atmospheric_sciences

Geography

Demography

Geology
$2.528035 \mathrm{e}-01$

$-7.976077 \mathrm{e}-02$

$-2.793733 \mathrm{e}-01$

$-1.505434 \mathrm{e}-01$

$-5.403332 \mathrm{e}-02$

$-7.577515 \mathrm{e}-01$

$1.550535 \mathrm{e}-01$

$-1.944527 \mathrm{e}-01$

$2.942587 \mathrm{e}-02$

$3.683397 \mathrm{e}-02$

$1.175878 \mathrm{e}-02$

$-3.770012 \mathrm{e}-02$

$-3.625727 \mathrm{e}-01$

$-1.220393 \mathrm{e}-01$

$-3.167762 \mathrm{e}-01$

$-1.207421 \mathrm{e}-01$

$3.054862 \mathrm{e}-01$

4.684407e-03

$1.626963 \mathrm{e}-01$

$4.096613 \mathrm{e}-02$

$1.237673 \mathrm{e}-01$

$-6.679883 \mathrm{e}-02$

4.149017e-02

$1.437026 \mathrm{e}-02$

$-5.832284 \mathrm{e}-01$

$2.161389 \mathrm{e}-01$

$-7.382883 \mathrm{e}-02$

$6.952355 \mathrm{e}-01$

$8.237331 \mathrm{e}-02$

$1.024956 \mathrm{e}-01$

$-1.369272 \mathrm{e}-02$

$-1.214073 e+00$

$3.169118 \mathrm{e}-02$

$-1.458771 \mathrm{e}-01$

$1.458212 \mathrm{e}-01$

$2.030989 \mathrm{e}-01$

$-2.357244 \mathrm{e}-01$

$-1.287140 \mathrm{e}-02$

$1.967876 \mathrm{e}-01$

$-2.133652 \mathrm{e}-01$

$-6.265838 \mathrm{e}-02$

$-1.611868 \mathrm{e}-01$

$1.304456 \mathrm{e}-02$

8.438197e-02

$-2.201872 \mathrm{e}-01$

$-1.493249 \mathrm{e}-01$

$8.962791 \mathrm{e}-02$

$-8.468416 \mathrm{e}-03$

9.070337e-02

$-4.088290 \mathrm{e}-02$

$-3.310122 \mathrm{e}-02$

$-1.433171 \mathrm{e}-01$

$1.478488 \mathrm{e}-01$

$3.218247 \mathrm{e}-02$

4.057837e-01

$3.488985 \mathrm{e}-01$

$3.253989 \mathrm{e}-01$

$4.519898 \mathrm{e}-01$

$2.752909 \mathrm{e}-03$

$-5.297603 \mathrm{e}-01$

$2.145436 \mathrm{e}-01$

$-6.302632 \mathrm{e}-02$

$-3.362137 \mathrm{e}-02$

$-2.139657 \mathrm{e}-01$

$2.239209 \mathrm{e}-01$

$-3.389201 \mathrm{e}-01$

$-1.519203 \mathrm{e}-01$

$3.328585 \mathrm{e}-02$

$-7.380299 \mathrm{e}-02$

$3.815501 \mathrm{e}-02$

$6.968550 \mathrm{e}-02$

$9.492276 \mathrm{e}-02$

$2.527153 \mathrm{e}-01$

$3.543971 \mathrm{e}-02$

4.829764e-02

$3.486413 \mathrm{e}-01$

$1.597574 \mathrm{e}-01$

$1.814369 \mathrm{e}-01$

$9.097205 \mathrm{e}-02$

$6.258772 \mathrm{e}-02$ 
344 Certain_Probability

345 Certain_Probability

346 Certain_Probability

347 Certain_Probability

348 Certain_Probability

349 Certain_Probability

350 Certain_Probability

351 Certain_Probability

352 Certain_Probability

353 Certain_Probability

354 Certain_Probability

355 Certain_Probability

356 Certain_Probability

357 Certain_Probability

358 Certain_Probability

359 Certain_Probability

360 Certain_Condition

361 Certain_Condition

362 Certain_Condition

363 Certain_Condition

364 Certain_Condition

365 Certain_Condition

366 Certain_Condition

367 Certain_Condition

368 Certain_Condition

369 Certain_Condition

370 Certain_Condition

371 Certain_Condition

372 Certain Condition

373 Certain_Condition

374 Certain_Condition

375 Certain_Condition

376 Certain Condition

377 Certain_Condition

378 Certain Condition

379 Certain_Condition

380 Certain_Condition

381 Certain_Condition

382 Certain_Condition

383 Certain_Condition

384 Certain_Condition

385 Certain_Condition

386 Certain_Condition

387 Certain_Condition

388 Certain Condition

389 Certain_Condition

390 Certain Condition

391 Certain_Condition

392 Certain Condition

393 Certain_Condition

394 Certain_Condition

395 Certain_Condition

396 Certain_Condition

397 Certain_Condition

398 Certain_Condition

399 Certain_Condition

400 Certain_Condition

401 Certain_Condition

402 Certain_Condition

403 Certain_Condition

404 Certain_Condition

405 Certain_Condition

406 Certain_Condition

407 Certain_Condition

408 Certain Condition

409 Certain_Condition

410 Certain_Condition

411 Certain_Condition

412 Certain_Condition
Medicine

Family_medicine

Evolutionary_biology

Astrobiology

Astronomy

Astrophysics

Geochemistry

Business

Finance

Management_science

Social_psychology

Political_science

Archaeology

Economics

Paleontology

Criminology

Intercept

source[T.news]

finding_len

flesch_reading_ease

first_author_rank

affi_rank

num_authors

journal_impact

Law

Condensed_matter_physics

Biomedical_engineering

Hydrology

Atomic_physics

Development_economics

Optics

Public_policy

Lung_cancer

Electronic_engineering

Artificial_intelligence

Machine_learning

Gender_studies

Chemical_engineering

Biophysics

Analytical_chemistry

Computer_science

Radiology

Urology

Architecture

Quantum mechanics

Particle_physics

Materials_science

Computational_biology

Agronomy

Environmental_resource management

Molecular_biology

Dermatology

Engineering

Surgery

Public health

Molecular_physics

Bioinformatics

Physics

Cognitive_psychology

Oncology

Pathology

Biochemistry

Social_science

Climatology

Pharmacology

Agriculture

Neuroscience

Fishery

Virology
$-3.045784 \mathrm{e}-01$

$-1.332693 \mathrm{e}-01$

4.378143e-02

$-3.428794 \mathrm{e}-01$

$-4.474937 \mathrm{e}-01$

$4.396899 \mathrm{e}-01$

$-4.068889 \mathrm{e}-01$

$-2.516853 \mathrm{e}-01$

$-5.942051 \mathrm{e}-01$

$1.649390 \mathrm{e}-12$

$1.217276 \mathrm{e}-01$

$-8.910235 \mathrm{e}-03$

$4.545359 \mathrm{e}-02$

$-2.313705 \mathrm{e}-01$

$-2.146638 \mathrm{e}-01$

$4.886135 \mathrm{e}-01$

$9.776916 \mathrm{e}-02$

$9.577395 \mathrm{e}-03$

$7.910887 \mathrm{e}-04$

$-1.082471 \mathrm{e}-04$

$-2.340008 \mathrm{e}-06$

$1.260599 \mathrm{e}-09$

$-3.395863 \mathrm{e}-04$

$-2.897048 \mathrm{e}-05$

7.879067e-02

$-5.647359 \mathrm{e}-02$

$-9.133277 \mathrm{e}-02$

$-2.043647 \mathrm{e}-02$

$-8.560960 \mathrm{e}-02$

$-1.207499 \mathrm{e}-01$

$-9.524136 \mathrm{e}-02$

$2.634518 \mathrm{e}-01$

$4.435076 \mathrm{e}-02$

$-1.107907 \mathrm{e}-01$

$-2.591684 \mathrm{e}-01$

6.191988e-01

$-1.239457 \mathrm{e}-02$

$2.628615 \mathrm{e}-01$

$-4.720782 \mathrm{e}-02$

$-9.921090 \mathrm{e}-02$

$1.392071 \mathrm{e}-02$

$4.483246 \mathrm{e}-03$

$-1.024062 \mathrm{e}-02$

$-9.140980 \mathrm{e}-02$

$-1.312127 \mathrm{e}-01$

$1.314888 \mathrm{e}-03$

$-8.966622 \mathrm{e}-02$

$-7.766247 \mathrm{e}-02$

$-5.676933 \mathrm{e}-02$

$2.139994 \mathrm{e}-02$

$-2.461304 \mathrm{e}-02$

$-3.553819 \mathrm{e}-02$

$-1.302261 \mathrm{e}-01$

$-7.269878 \mathrm{e}-02$

$2.573100 \mathrm{e}-02$

$-8.664672 \mathrm{e}-02$

$-7.633961 \mathrm{e}-02$

$-2.141574 \mathrm{e}-02$

$-3.092240 \mathrm{e}-02$

$5.231722 \mathrm{e}-02$

$-5.046651 \mathrm{e}-02$

$-3.227280 \mathrm{e}-02$

$-2.146332 \mathrm{e}-01$

$1.172965 \mathrm{e}-03$

$4.859199 \mathrm{e}-02$

$-5.938870 \mathrm{e}-02$

$-3.513996 \mathrm{e}-03$

$-2.060256 \mathrm{e}-02$

$-1.915763 \mathrm{e}-02$ 8.358695e-02

$4.179014 \mathrm{e}-02$

$1.042324 \mathrm{e}-01$

$2.219312 \mathrm{e}-01$

$1.664975 \mathrm{e}-01$

$2.568342 \mathrm{e}-01$

$2.493150 \mathrm{e}-01$

$1.423630 \mathrm{e}-01$

$1.508111 \mathrm{e}-01$

$2.417729 \mathrm{e}-12$

$4.881799 \mathrm{e}-02$

$2.268015 \mathrm{e}-01$

$2.205702 \mathrm{e}-01$

$1.114151 \mathrm{e}-01$

$1.364687 \mathrm{e}-01$

$3.178086 \mathrm{e}-01$

$2.176721 \mathrm{e}-02$

$3.058244 \mathrm{e}-03$

$1.725983 \mathrm{e}-04$

$6.884332 \mathrm{e}-05$

$8.815408 \mathrm{e}-07$

$8.791888 \mathrm{e}-07$

$1.198636 \mathrm{e}-04$

$1.235831 \mathrm{e}-04$

$5.994242 \mathrm{e}-02$

$1.221277 \mathrm{e}-01$

$1.153814 \mathrm{e}-01$

$1.011987 \mathrm{e}-01$

$1.436410 \mathrm{e}-01$

$1.634597 \mathrm{e}-01$

$1.783310 \mathrm{e}-01$

$1.097177 \mathrm{e}-01$

$2.604677 \mathrm{e}-02$

$2.997073 \mathrm{e}-01$

$1.515518 \mathrm{e}-01$

$1.605394 \mathrm{e}-01$

9.651916e-02

$1.126731 \mathrm{e}-01$

$6.912797 \mathrm{e}-02$

$1.225813 \mathrm{e}-01$

$8.073860 \mathrm{e}-02$

$2.877305 \mathrm{e}-02$

4.350257e-02

$9.877068 \mathrm{e}-02$

$3.912087 \mathrm{e}-01$

$3.385346 \mathrm{e}-01$

$5.834960 \mathrm{e}-02$

$1.436528 \mathrm{e}-01$

7.691043e-02

4.436444e-02

$2.873449 \mathrm{e}-02$

$3.914095 \mathrm{e}-02$

$1.042060 \mathrm{e}-01$

$1.461340 \mathrm{e}-02$

$1.991530 \mathrm{e}-02$

$1.437606 \mathrm{e}-01$

$6.587520 \mathrm{e}-02$

$7.481463 \mathrm{e}-02$

$3.751189 \mathrm{e}-02$

$2.580775 \mathrm{e}-02$

$2.676687 \mathrm{e}-02$

$3.665564 \mathrm{e}-02$

$1.459003 \mathrm{e}-01$

$4.052698 \mathrm{e}-02$

$5.850057 \mathrm{e}-02$

4.728087e-02

$3.145883 \mathrm{e}-02$

6.731171e-02

$3.035278 \mathrm{e}-02$

$2.696304 \mathrm{e}-04$

$1.430970 \mathrm{e}-03$

$6.744657 \mathrm{e}-01$

$1.223753 \mathrm{e}-01$

$7.203928 \mathrm{e}-03$

$8.692783 \mathrm{e}-02$

$1.026978 \mathrm{e}-01$

$7.709892 \mathrm{e}-02$

8.188490e-05

$4.951207 \mathrm{e}-01$

$1.266140 \mathrm{e}-02$

$9.686626 \mathrm{e}-01$

8.367370e-01 
413 Certain_Condition

414 Certain_Condition

415 Certain_Condition

416 Certain_Condition

417 Certain_Condition

418 Certain_Condition

419 Certain_Condition

420 Certain_Condition

421 Certain_Condition

422 Certain_Condition

423 Certain_Condition

424 Certain_Condition

425 Certain_Condition

426 Certain_Condition

427 Certain_Condition

428 Certain_Condition

429 Certain_Condition

430 Certain_Condition

431 Certain_Condition

432 Certain_Condition

433 Certain_Condition

434 Certain_Condition

435 Certain_Condition

436 Certain_Condition

437 Certain_Condition

438 Certain_Condition

439 Certain_Condition

440 Certain_Condition

441 Certain_Condition

442 Certain_Condition

443 Certain_Condition

444 Certain_Condition

445 Certain Condition

446 Certain_Condition

447 Certain Condition

448 Certain_Condition

449 Certain_Condition

450 Certain_Condition

451 Certain_Condition

452 Certain_Condition

453 Certain_Condition

454 Certain_Condition

455 Certain_Condition

456 Certain_Condition

457 Certain Condition

458 Certain_Condition

459 Certain_Condition

460 Certain_Condition

461 Certain Condition

462 Certain_Condition

463 Certain_Condition

464 Certain_Condition

465 Certain_Condition

466 Certain_Condition

467 Certain_Condition

468 Certain_Condition

469 Certain_Condition

470 Certain_Condition

471 Certain_Condition

472 Certain_Condition

473 Certain_Condition

474 Certain_Condition

475 Certain_Condition

476 Certain_Condition

477 Certain_Condition

478 Certain_Condition

479 Certain_Condition

480 Certain_Suggestion

481 Certain_Suggestion

482 Certain_Suggestion
Microbiology

Communication

Endocrinology

Epigenetics

Marketing

Global_health

Agroforestry

Chemistry

Mathematics

Gynecology

Gerontology

Meteorology

Veterinary_medicine

Government

Dentistry

Microeconomics

Sociology

Emergency_medicine

Pediatrics

Advertising

Oceanography

Developmental_psychology

Internal_medicine

Cardiology

Environmental_engineering

General_surgery

Environmental_science

Geophysics

Immunology

Clinical_psychology

Toxicology

Ecology

Biology

Epidemiology

Physical_therapy

Physiology

Statistics

Psychopathology

Animal_science

Nursing

Seismology

History

Psychiatry

Zoology

Psychology

Etiology

Geomorphology

Atmospheric_sciences

Geography

Demography

Geology

Medicine

Family_medicine

Evolutionary_biology

Astrobiology

Astronomy

Astrophysics

Geochemistry

Business

Finance

Management_science

Social_psychology

Political_science

Archaeology

Economics

Paleontology

Criminology

Intercept

source[T.news]

finding len 6.088217e-02

$2.784153 \mathrm{e}-01$

$-2.988003 \mathrm{e}-02$

$3.062971 \mathrm{e}-02$

$-5.156308 \mathrm{e}-02$

$1.247554 \mathrm{e}-01$

$-1.285461 \mathrm{e}-01$

$-1.958057 \mathrm{e}-01$

$-3.481756 \mathrm{e}-01$

$2.740499 \mathrm{e}-02$

$-4.481767 \mathrm{e}-02$

$-2.454682 \mathrm{e}-01$

$-9.408361 \mathrm{e}-02$

$-9.671124 \mathrm{e}-02$

$-6.633945 \mathrm{e}-02$

$-1.839182 \mathrm{e}-01$

$7.075854 \mathrm{e}-02$

$5.873041 \mathrm{e}-03$

$-4.453224 \mathrm{e}-02$

$-1.297368 \mathrm{e}-01$

$-1.678544 \mathrm{e}-01$

7.400457e-02

$-4.533502 \mathrm{e}-02$

$2.001239 \mathrm{e}-02$

$-1.842664 \mathrm{e}-01$

$-5.081619 \mathrm{e}-02$

$1.036854 \mathrm{e}-01$

$4.309711 \mathrm{e}-01$

$-1.602773 \mathrm{e}-02$

$-6.853400 \mathrm{e}-02$

$3.852756 \mathrm{e}-01$

$-2.117069 \mathrm{e}-04$

$-1.224078 \mathrm{e}-01$

6.585129e-02

$-3.128475 \mathrm{e}-02$

$-7.763389 \mathrm{e}-02$

$-1.690406 \mathrm{e}-01$

$-7.429194 \mathrm{e}-02$

$-5.952692 \mathrm{e}-02$

$-4.568915 \mathrm{e}-02$

$-1.083419 \mathrm{e}-02$

$-1.645156 \mathrm{e}-01$

$-1.243366 \mathrm{e}-02$

4.381916e-02

$-6.183735 \mathrm{e}-02$

$-6.847151 \mathrm{e}-02$

$-1.541098 \mathrm{e}-01$

$-1.274566 \mathrm{e}-01$

$2.070757 \mathrm{e}-01$

7.114067e-02

$3.239898 \mathrm{e}-02$

$-8.602196 \mathrm{e}-02$

$-3.798406 \mathrm{e}-02$

$2.446438 \mathrm{e}-02$

$-1.853467 \mathrm{e}-01$

$-1.098202 \mathrm{e}-01$

$-6.442768 \mathrm{e}-02$

$-1.622523 \mathrm{e}-01$

$1.974802 \mathrm{e}-02$

$-1.195103 \mathrm{e}-01$

$2.057818 \mathrm{e}-12$

$-7.944953 \mathrm{e}-03$

$-5.904802 \mathrm{e}-02$

$-4.046136 \mathrm{e}-02$

$1.861374 \mathrm{e}-03$

$-7.289507 \mathrm{e}-02$

$1.582040 \mathrm{e}-01$

$1.337516 \mathrm{e}-02$

$2.060145 \mathrm{e}-03$

$-2.033606 \mathrm{e}-04$ 4.514764e-02

4.636010e-02

$1.494913 \mathrm{e}-02$

4.477421e-02

4.830033e-02

$7.095151 \mathrm{e}-02$

$6.199269 \mathrm{e}-02$

$5.178371 \mathrm{e}-02$

$1.509125 \mathrm{e}-01$

$2.475815 \mathrm{e}-02$

$2.623861 \mathrm{e}-02$

$7.844692 \mathrm{e}-02$

$1.790860 \mathrm{e}-01$

$5.266890 \mathrm{e}-02$

9.709821e-02

$8.253127 \mathrm{e}-02$

$1.029855 \mathrm{e}-01$

$2.195756 \mathrm{e}-02$

$1.763736 \mathrm{e}-02$

4.779750e-02

$7.284787 \mathrm{e}-02$

$2.633660 \mathrm{e}-02$

$1.440825 \mathrm{e}-02$

2.292687e-02

$6.913604 \mathrm{e}-02$

$2.673841 \mathrm{e}-02$

5.446312e-02

$1.413982 \mathrm{e}-01$

$1.890673 \mathrm{e}-02$

$2.729221 \mathrm{e}-02$

8.810637e-02

$2.102523 \mathrm{e}-02$

4.030791e-02

$2.213267 \mathrm{e}-02$

$1.439848 \mathrm{e}-02$

5.559271e-02

$1.779458 \mathrm{e}-01$

6.452921e-02

$1.008103 \mathrm{e}-01$

$2.615064 \mathrm{e}-02$

$1.105837 \mathrm{e}-01$

$3.094188 \mathrm{e}-01$

$1.833197 \mathrm{e}-02$

4.807953e-02

$3.999994 \mathrm{e}-02$

$5.128160 \mathrm{e}-02$

$6.032463 \mathrm{e}-02$

$6.472682 \mathrm{e}-02$

$5.563179 \mathrm{e}-02$

$1.690390 \mathrm{e}-02$

$6.048011 \mathrm{e}-02$

$3.446668 \mathrm{e}-02$

$1.723197 \mathrm{e}-02$

$4.297975 \mathrm{e}-02$

$9.151229 \mathrm{e}-02$

$6.865445 \mathrm{e}-02$

$1.059044 \mathrm{e}-01$

$1.028038 \mathrm{e}-01$

$5.870270 \mathrm{e}-02$

$6.218625 \mathrm{e}-02$

9.969393e-13

$2.012987 \mathrm{e}-02$

9.352052e-02

$9.095106 \mathrm{e}-02$

$4.594149 \mathrm{e}-02$

$5.627223 \mathrm{e}-02$

$1.310469 \mathrm{e}-01$

$1.628080 \mathrm{e}-02$

$2.287416 \mathrm{e}-03$

$1.290950 \mathrm{e}-04$

$1.775169 \mathrm{e}-01$

$1.958185 \mathrm{e}-09 \quad * * *$

$4.565276 \mathrm{e}-02$ *

$4.939287 \mathrm{e}-01$

$2.857427 \mathrm{e}-01$

$7.871654 \mathrm{e}-02$

$3.813903 \mathrm{e}-02$

$1.567567 \mathrm{e}-04$

$2.106285 \mathrm{e}-02$

$2.683543 \mathrm{e}-01$ 
483 Certain_Suggestion

484 Certain_Suggestion

485 Certain_Suggestion

486 Certain_Suggestion

487 Certain_Suggestion

488 Certain_Suggestion

489 Certain_Suggestion

490 Certain_Suggestion

491 Certain_Suggestion

492 Certain_Suggestion

493 Certain_Suggestion

494 Certain_Suggestion

495 Certain_Suggestion

496 Certain_Suggestion

497 Certain_Suggestion

498 Certain_Suggestion

499 Certain_Suggestion

500 Certain_Suggestion

501 Certain_Suggestion

502 Certain_Suggestion

503 Certain_Suggestion

504 Certain_Suggestion

505 Certain_Suggestion

506 Certain_Suggestion

507 Certain_Suggestion

508 Certain_Suggestion

509 Certain_Suggestion

510 Certain_Suggestion

511 Certain_Suggestion

512 Certain_Suggestion

513 Certain_Suggestion

514 Certain_Suggestion

515 Certain_Suggestion

516 Certain_Suggestion

517 Certain_Suggestion

518 Certain_Suggestion

519 Certain_Suggestion

520 Certain_Suggestion

521 Certain_Suggestion

522 Certain_Suggestion

523 Certain_Suggestion

524 Certain_Suggestion

525 Certain_Suggestion

526 Certain_Suggestion

527 Certain_Suggestion

528 Certain_Suggestion

529 Certain_Suggestion

530 Certain_Suggestion

531 Certain_Suggestion

532 Certain_Suggestion

533 Certain_Suggestion

534 Certain_Suggestion

535 Certain_Suggestion

536 Certain_Suggestion

537 Certain_Suggestion

538 Certain_Suggestion

539 Certain_Suggestion

540 Certain_Suggestion

541 Certain_Suggestion

542 Certain_Suggestion

543 Certain_Suggestion

544 Certain_Suggestion

545 Certain_Suggestion

546 Certain_Suggestion

547 Certain_Suggestion

548 Certain_Suggestion

549 Certain_Suggestion

550 Certain_Suggestion

551 Certain_Suggestion flesch_reading_ease

first_author_rank

affi_rank

num_authors

journal_impact

Law

Condensed_matter_physics

Biomedical_engineering

Hydrology

Atomic_physics

Development_economics

Optics

Public_policy

Lung_cancer

Electronic_engineering

Artificial_intelligence

Machine_learning

Gender_studies

Chemical_engineering

Biophysics

Analytical_chemistry

Computer_science

Radiology

Urology

Architecture

Quantum_mechanics

Particle physics

Materials_science

Computational_biology

Agronomy

Environmental_resource management

Molecular_biology

Dermatology

Engineering

Surgery

Public_health

Molecular_physics

Bioinformatics

Physics

Cognitive_psychology

Oncology

Pathology

Biochemistry

Social_science

Climatology

Pharmacology

Agriculture

Neuroscience

Fishery

Virology

Microbiology

Communication

Endocrinology

Epigenetics

Marketing

Global_health

Agroforestry

Chemistry

Mathematics

Gynecology

Gerontology

Meteorology

Veterinary_medicine

Government

Dentistry

Microeconomics

Sociology

Emergency_medicine

Pediatrics
$-2.718805 \mathrm{e}-04$

$-1.291753 \mathrm{e}-06$

$1.356428 \mathrm{e}-06$

$3.116718 \mathrm{e}-05$

$-2.090195 \mathrm{e}-04$

$1.435503 \mathrm{e}-02$

$1.763714 \mathrm{e}-01$

$-2.298391 \mathrm{e}-02$

$-5.101807 \mathrm{e}-02$

$3.176237 \mathrm{e}-03$

$-1.103778 \mathrm{e}-01$

$2.045380 \mathrm{e}-02$

$4.569272 \mathrm{e}-01$

$5.162150 \mathrm{e}-02$

$-3.335916 \mathrm{e}-03$

$-3.902308 \mathrm{e}-02$

$-3.056129 \mathrm{e}-03$

$-4.195338 \mathrm{e}-02$

$-4.309337 \mathrm{e}-03$

$-1.753191 \mathrm{e}-02$

$-6.624190 \mathrm{e}-03$

$5.580729 \mathrm{e}-02$

$-1.307528 \mathrm{e}-02$

$-4.964304 \mathrm{e}-02$

$-4.133132 \mathrm{e}-02$

$-6.877816 \mathrm{e}-03$

$2.657022 \mathrm{e}-02$

$4.838278 \mathrm{e}-02$

$-2.876853 \mathrm{e}-02$

$-1.159018 \mathrm{e}-01$

$1.571620 \mathrm{e}-01$

$-7.512474 \mathrm{e}-03$

$-5.013059 \mathrm{e}-02$

$1.462886 \mathrm{e}-02$

$-2.438935 \mathrm{e}-02$

$4.845218 \mathrm{e}-02$

$-4.709223 \mathrm{e}-02$

$-4.581454 \mathrm{e}-02$

$2.471097 \mathrm{e}-02$

$1.487764 \mathrm{e}-02$

$-2.155058 \mathrm{e}-02$

$6.062855 \mathrm{e}-02$

$3.071896 \mathrm{e}-02$

$7.472158 \mathrm{e}-02$

$6.987124 \mathrm{e}-02$

$4.527395 \mathrm{e}-02$

5.852581e-02

$-1.857024 \mathrm{e}-03$

$-2.098984 \mathrm{e}-02$

$4.465529 \mathrm{e}-04$

$-8.746573 \mathrm{e}-03$

$2.886112 \mathrm{e}-02$

$-1.474743 \mathrm{e}-02$

$-2.668680 \mathrm{e}-02$

$4.810229 \mathrm{e}-03$

$-6.887376 \mathrm{e}-02$

$1.534798 \mathrm{e}-01$

$9.707114 \mathrm{e}-02$

$9.135101 \mathrm{e}-03$

$3.006455 \mathrm{e}-03$

$-1.991193 \mathrm{e}-02$

$-6.713493 \mathrm{e}-02$

$2.969761 \mathrm{e}-01$

$-4.999288 \mathrm{e}-02$

$-3.338680 \mathrm{e}-02$

2.971161e-01

$2.453899 \mathrm{e}-01$

$-4.259541 \mathrm{e}-02$

$4.789542 \mathrm{e}-03$ 5.149141e-05 $6.593491 \mathrm{e}-07$ $6.575899 \mathrm{e}-07$ $8.965205 \mathrm{e}-05$ 9.243404e-05 4.483397e-02 $9.134549 \mathrm{e}-02$ $8.629962 \mathrm{e}-02$ $7.569164 \mathrm{e}-02$ $1.074364 \mathrm{e}-01$

$1.222598 \mathrm{e}-01$

$1.333828 \mathrm{e}-01$

$8.206346 \mathrm{e}-02$

$1.948170 \mathrm{e}-02$

$2.241663 \mathrm{e}-01$

$1.133533 \mathrm{e}-01$

$1.200756 \mathrm{e}-01$

7.219157e-02

$8.427394 \mathrm{e}-02$

$5.170432 \mathrm{e}-02$

$9.168481 \mathrm{e}-02$

$6.038849 \mathrm{e}-02$

$2.152083 \mathrm{e}-02$

$3.253778 \mathrm{e}-02$

$7.387560 \mathrm{e}-02$

$2.926048 \mathrm{e}-01$

$2.532072 \mathrm{e}-01$

$4.364263 \mathrm{e}-02$

$1.074452 \mathrm{e}-01$

$5.752521 \mathrm{e}-02$

3.318242e-02

$2.149198 \mathrm{e}-02$

$2.927550 \mathrm{e}-02$

$7.794092 \mathrm{e}-02$

$1.093010 \mathrm{e}-02$

$1.489567 \mathrm{e}-02$

$1.075258 \mathrm{e}-01$

4.927141e-02

$5.595766 \mathrm{e}-02$

$2.805705 \mathrm{e}-02$

$1.930292 \mathrm{e}-02$

$2.002030 \mathrm{e}-02$

$2.741661 \mathrm{e}-02$

$1.091262 \mathrm{e}-01$

$3.031218 \mathrm{e}-02$

$4.375554 \mathrm{e}-02$

$3.536376 \mathrm{e}-02$

$2.352966 \mathrm{e}-02$

$5.034584 \mathrm{e}-02$

$2.270239 \mathrm{e}-02$

$3.376821 \mathrm{e}-02$

$3.467507 \mathrm{e}-02$

$1.118121 \mathrm{e}-02$

$3.348890 \mathrm{e}-02$

$3.612627 \mathrm{e}-02$

$5.306823 \mathrm{e}-02$

$4.636748 \mathrm{e}-02$

$3.873166 \mathrm{e}-02$

$1.128751 \mathrm{e}-01$

$1.851788 \mathrm{e}-02$

$1.962518 \mathrm{e}-02$

5.867443e-02

$1.339475 \mathrm{e}-01$

$3.939374 \mathrm{e}-02$

$7.262468 \mathrm{e}-02$

$6.172932 \mathrm{e}-02$

$7.702808 \mathrm{e}-02$

$1.642317 \mathrm{e}-02$

$1.319188 \mathrm{e}-02$

$1.311762 \mathrm{e}-07$

$5.011832 \mathrm{e}-02$

$3.915834 \mathrm{e}-02$

$7.281116 \mathrm{e}-01$

$2.375816 \mathrm{e}-02$

$7.488356 \mathrm{e}-01$

$5.352734 \mathrm{e}-02$

$7.899917 \mathrm{e}-01$

$5.003072 \mathrm{e}-01$

$9.764153 \mathrm{e}-01$

$3.666414 \mathrm{e}-01$

$8.781273 \mathrm{e}-01$

$2.628069 \mathrm{e}-08$ 
552 Certain_Suggestion

553 Certain_Suggestion

554 Certain_Suggestion

555 Certain_Suggestion

556 Certain_Suggestion

557 Certain_Suggestion

558 Certain_Suggestion

559 Certain_Suggestion

560 Certain_Suggestion

561 Certain_Suggestion

562 Certain_Suggestion

563 Certain_Suggestion

564 Certain_Suggestion

565 Certain_Suggestion

566 Certain_Suggestion

567 Certain_Suggestion

568 Certain_Suggestion

569 Certain_Suggestion

570 Certain_Suggestion

571 Certain_Suggestion

572 Certain_Suggestion

573 Certain_Suggestion

574 Certain_Suggestion

575 Certain_Suggestion

576 Certain_Suggestion

577 Certain_Suggestion

578 Certain_Suggestion

579 Certain_Suggestion

580 Certain_Suggestion

581 Certain_Suggestion

582 Certain_Suggestion

583 Certain_Suggestion

584 Certain_Suggestion

585 Certain_Suggestion

586 Certain_Suggestion

587 Certain_Suggestion

588 Certain_Suggestion

589 Certain_Suggestion

590 Certain_Suggestion

591 Certain_Suggestion

592 Certain_Suggestion

593 Certain_Suggestion

594 Certain_Suggestion

595 Certain_Suggestion

596 Certain_Suggestion

597 Certain_Suggestion

598 Certain_Suggestion

599 Certain_Suggestion

600 Certain_Framing

601 Certain_Framing

602 Certain_Framing

603 Certain_Framing

604 Certain_Framing

605 Certain_Framing

606 Certain_Framing

607 Certain_Framing

608 Certain_Framing

609 Certain_Framing

610 Certain_Framing

611 Certain_Framing

612 Certain_Framing

613 Certain_Framing

614 Certain_Framing

615 Certain_Framing

616 Certain_Framing

617 Certain_Framing

618 Certain_Framing

619 Certain_Framing

620 Certain_Framing

621 Certain_Framing
Advertising

Oceanography

Developmental_psychology

Internal_medicine

Cardiology

Environmental_engineering

General_surgery

Environmental_science

Geophysics

Immunology

Clinical_psychology

Toxicology

Ecology

Biology

Epidemiology

Physical_therapy

Physiology

Statistics

Psychopathology

Animal_science

Nursing

Seismology

History

Psychiatry

Zoology

Psychology

Etiology

Geomorphology

Atmospheric sciences

Geography

Demography

Geology

Medicine

Family_medicine

Evolutionary_biology

Astrobiology

Astronomy

Astrophysics

Geochemistry

Business

Finance

Management_science

Social_psychology

Political_science

Archaeology

Economics

Paleontology

Criminology

Intercept

source[T.news]

finding_len

flesch_reading_ease

first_author_rank

affi_rank

num_authors

journal_impact

Law

Condensed_matter_physics

Biomedical_engineering

Hydrology

Atomic_physics

Development_economics

Optics

Public_policy

Lung_cancer

Electronic_engineering

Artificial_intelligence

Machine_learning

Gender_studies

Chemical_engineering
$-7.780238 \mathrm{e}-02$

$-4.733839 \mathrm{e}-02$

$8.300195 \mathrm{e}-03$

$-2.176705 \mathrm{e}-02$

$-1.771320 \mathrm{e}-02$

$4.958653 \mathrm{e}-02$

4.301141e-03

$1.049909 \mathrm{e}-01$

$-6.289009 \mathrm{e}-02$

$2.535643 \mathrm{e}-02$

$-9.793246 \mathrm{e}-04$

$-5.211729 \mathrm{e}-02$

$2.409142 \mathrm{e}-02$

$7.394016 \mathrm{e}-02$

$-2.551913 \mathrm{e}-02$

$4.943949 \mathrm{e}-03$

$-4.855773 \mathrm{e}-02$

$-1.388574 \mathrm{e}-02$

$-3.110469 \mathrm{e}-02$

$-2.025005 \mathrm{e}-02$

$-2.629332 \mathrm{e}-02$

$-2.970905 \mathrm{e}-02$

$2.448557 \mathrm{e}-02$

$-1.296226 \mathrm{e}-02$

$7.885082 \mathrm{e}-02$

$5.634250 \mathrm{e}-02$

$4.706578 \mathrm{e}-02$

$8.693252 \mathrm{e}-02$

$2.657336 \mathrm{e}-03$

$1.059079 \mathrm{e}-01$

$-1.544552 \mathrm{e}-02$

$5.146969 \mathrm{e}-02$

$9.556823 \mathrm{e}-02$

$2.090977 \mathrm{e}-02$

$5.480893 \mathrm{e}-02$

$3.374449 \mathrm{e}-01$

$-1.343625 \mathrm{e}-02$

$3.376346 \mathrm{e}-02$

$-1.703213 \mathrm{e}-02$

$1.435940 \mathrm{e}-01$

$1.580820 \mathrm{e}-01$

$-5.145384 \mathrm{e}-14$

$-2.570641 \mathrm{e}-03$

$4.885032 \mathrm{e}-02$

$-5.425985 \mathrm{e}-02$

$5.059419 \mathrm{e}-02$

$-5.547359 \mathrm{e}-02$

$7.128781 \mathrm{e}-05$

$1.768614 \mathrm{e}-01$

$-6.653818 \mathrm{e}-02$

$1.798119 \mathrm{e}-03$

$-7.113732 \mathrm{e}-04$

$-2.910100 \mathrm{e}-06$

$-3.550267 \mathrm{e}-07$

$-1.513935 \mathrm{e}-04$

$9.591003 \mathrm{e}-05$

$-3.606114 \mathrm{e}-02$

7.905256e-01

$2.956243 \mathrm{e}-01$

$6.364139 \mathrm{e}-01$

6.246608e-01

$-2.244286 \mathrm{e}-01$

$-3.144005 \mathrm{e}-01$

$3.549480 \mathrm{e}-03$

$4.472236 \mathrm{e}-02$

$-3.641664 \mathrm{e}-01$

$2.078497 \mathrm{e}-02$

$1.330748 \mathrm{e}-01$

$-2.281151 \mathrm{e}-01$

$-1.773623 \mathrm{e}-01$ 3.575017e-02

5.448662e-02

$1.969848 \mathrm{e}-02$

$1.077666 \mathrm{e}-02$

$1.714817 \mathrm{e}-02$

$5.171035 \mathrm{e}-02$

$1.999901 \mathrm{e}-02$

$4.073573 \mathrm{e}-02$

$1.057589 \mathrm{e}-01$

$1.414131 \mathrm{e}-02$

$2.041323 \mathrm{e}-02$

$6.589922 \mathrm{e}-02$

$1.572583 \mathrm{e}-02$

$3.014833 \mathrm{e}-02$

$1.655415 \mathrm{e}-02$

$1.076935 \mathrm{e}-02$

4.158061e-02

$1.330947 \mathrm{e}-01$

$4.826467 \mathrm{e}-02$

$7.540111 \mathrm{e}-02$

$1.955939 \mathrm{e}-02$

$8.271116 \mathrm{e}-02$

$2.314300 \mathrm{e}-01$

$1.371141 \mathrm{e}-02$

$3.596112 \mathrm{e}-02$

$2.991798 \mathrm{e}-02$

$3.835611 \mathrm{e}-02$

$4.511985 \mathrm{e}-02$

4.841247e-02

$4.160984 \mathrm{e}-02$

$1.264329 \mathrm{e}-02$

4.523614e-02

$2.577938 \mathrm{e}-02$

$1.288866 \mathrm{e}-02$

$3.214673 \mathrm{e}-02$

6.844668e-02

$5.135014 \mathrm{e}-02$

$7.921125 \mathrm{e}-02$

$7.689221 \mathrm{e}-02$

$4.390673 \mathrm{e}-02$

$4.651225 \mathrm{e}-02$

$7.456614 \mathrm{e}-13$

$1.505615 \mathrm{e}-02$

$6.994874 \mathrm{e}-02$

6.802691e-02

$3.436197 \mathrm{e}-02$

$4.208885 \mathrm{e}-02$

$9.801659 \mathrm{e}-02$

$3.177267 \mathrm{e}-02$

$4.463989 \mathrm{e}-03$

$2.519344 \mathrm{e}-04$

$1.004877 \mathrm{e}-04$

$1.286748 \mathrm{e}-06$

$1.283315 \mathrm{e}-06$

$1.749598 \mathrm{e}-04$

$1.803890 \mathrm{e}-04$

$8.749540 \mathrm{e}-02$

$1.782646 \mathrm{e}-01$

$1.684174 \mathrm{e}-01$

$1.477154 \mathrm{e}-01$

$2.096667 \mathrm{e}-01$

$2.385952 \mathrm{e}-01$

$2.603022 \mathrm{e}-01$

$1.601503 \mathrm{e}-01$

3.801937e-02

$4.374700 \mathrm{e}-01$

$2.212138 \mathrm{e}-01$

$2.343326 \mathrm{e}-01$

$1.408849 \mathrm{e}-01$

$1.644642 \mathrm{e}-01$

2.955223e-02 $3.849683 \mathrm{e}-01$ $6.734975 \mathrm{e}-01$ $4.342118 \mathrm{e}-02$

$3.016464 \mathrm{e}-01$

$3.376124 \mathrm{e}-01$

$8.297179 \mathrm{e}-01$

9.966501e-03

$5.520843 \mathrm{e}-01$

$7.298393 \mathrm{e}-02$ 
622 Certain_Framing

623 Certain_Framing

624 Certain_Framing

625 Certain_Framing

626 Certain_Framing

627 Certain_Framing

628 Certain_Framing

629 Certain_Framing

630 Certain_Framing

631 Certain_Framing

632 Certain_Framing

633 Certain_Framing

634 Certain_Framing

635 Certain_Framing

636 Certain_Framing

637 Certain_Framing

638 Certain_Framing

639 Certain_Framing

640 Certain_Framing

641 Certain_Framing

642 Certain_Framing

643 Certain_Framing

644 Certain_Framing

645 Certain_Framing

646 Certain_Framing

647 Certain_Framing

648 Certain_Framing

649 Certain_Framing

650 Certain_Framing

651 Certain_Framing

652 Certain_Framing

653 Certain_Framing

654 Certain_Framing

655 Certain_Framing

656 Certain_Framing

657 Certain_Framing

658 Certain_Framing

659 Certain_Framing

660 Certain_Framing

661 Certain_Framing

662 Certain_Framing

663 Certain_Framing

664 Certain_Framing

665 Certain_Framing

666 Certain_Framing

667 Certain_Framing

668 Certain_Framing

669 Certain_Framing

670 Certain_Framing

671 Certain_Framing

672 Certain_Framing

673 Certain_Framing

674 Certain_Framing

675 Certain_Framing

676 Certain_Framing

677 Certain_Framing

678 Certain_Framing

679 Certain_Framing

680 Certain_Framing

681 Certain_Framing

682 Certain_Framing

683 Certain_Framing

684 Certain_Framing

685 Certain_Framing

686 Certain_Framing

687 Certain_Framing

688 Certain_Framing

689 Certain_Framing

690 Certain_Framing
Biophysics

Analytical_chemistry

Computer_science

Radiology

Urology

Architecture

Quantum_mechanics

Particle_physics

Materials_science

Computational_biology

Agronomy

Environmental_resource management

Molecular_biology

Dermatology

Engineering

Surgery

Public_health

Molecular_physics

Bioinformatics

Physics

Cognitive_psychology

Oncology

Pathology

Biochemistry

Social_science

Climatology

Pharmacology

Agriculture

Neuroscience

Fishery

Virology

Microbiology

Communication

Endocrinology

Epigenetics

Marketing

Global_health

Agroforestry

Chemistry

Mathematics

Gynecology

Gerontology

Meteorology

Veterinary_medicine

Government

Dentistry

Microeconomics

Sociology

Emergency_medicine

Pediatrics

Advertising

Oceanography

Developmental_psychology

Internal_medicine

Cardiology

Environmental_engineering

General_surgery

Environmental_science

Geophysics

Immunology

Clinical_psychology

Toxicology

Ecology

Biology

Epidemiology

Physical_therapy

Physiology

Statistics

Psychopathology
$-2.215126 \mathrm{e}-01$

$9.256706 \mathrm{e}-02$

$8.466805 \mathrm{e}-02$

$1.817084 \mathrm{e}-02$

$-1.685674 \mathrm{e}-02$

$5.472754 \mathrm{e}-01$

6.205399e-01

$-7.372746 \mathrm{e}-01$

$1.228320 \mathrm{e}-01$

$-3.510084 \mathrm{e}-01$

$-9.498953 \mathrm{e}-02$

$-6.612410 \mathrm{e}-02$

$-1.270884 \mathrm{e}-02$

$-1.101070 \mathrm{e}-01$

$4.388128 \mathrm{e}-01$

$-5.267669 \mathrm{e}-02$

$-3.536222 \mathrm{e}-02$

$1.698111 \mathrm{e}-01$

$-8.723332 \mathrm{e}-02$

$-7.774521 \mathrm{e}-02$

$1.454097 \mathrm{e}-01$

$-1.477650 \mathrm{e}-02$

$-4.875385 \mathrm{e}-02$

4.624853e-02

$1.696629 \mathrm{e}-01$

$-1.510953 \mathrm{e}-01$

$2.293167 \mathrm{e}-01$

$-1.062199 \mathrm{e}-03$

$-1.753978 \mathrm{e}-01$

$9.172765 \mathrm{e}-02$

$-6.151043 e-02$

$-7.257720 \mathrm{e}-02$

$-1.395912 \mathrm{e}-01$

$-1.211106 \mathrm{e}-02$

$-1.002413 \mathrm{e}-01$

$2.184494 \mathrm{e}-04$

$-3.915400 \mathrm{e}-02$

$8.850096 \mathrm{e}-02$

$-3.275068 \mathrm{e}-02$

$-3.968767 \mathrm{e}-01$

$1.609573 \mathrm{e}-02$

$-4.474339 \mathrm{e}-02$

$1.489492 \mathrm{e}-01$

$-3.616296 \mathrm{e}-01$

$-1.025851 \mathrm{e}-01$

$-1.646786 \mathrm{e}-01$

$1.268712 \mathrm{e}-01$

$-1.769746 \mathrm{e}-01$

$-7.270091 \mathrm{e}-02$

$-4.859457 \mathrm{e}-02$

$1.864501 \mathrm{e}-02$

$1.285919 \mathrm{e}-01$

$-4.514817 \mathrm{e}-02$

$-5.697354 \mathrm{e}-02$

$-8.515402 \mathrm{e}-02$

4.198091e-02

$5.296179 \mathrm{e}-02$

$-9.601846 \mathrm{e}-02$

$2.878822 \mathrm{e}-01$

$-2.213069 \mathrm{e}-02$

$5.989462 \mathrm{e}-02$

$-1.701951 \mathrm{e}-01$

$-8.975301 \mathrm{e}-02$

$9.653216 \mathrm{e}-02$

$2.623765 \mathrm{e}-04$

$-1.041203 \mathrm{e}-02$

$2.457842 \mathrm{e}-01$

$1.104481 \mathrm{e}-01$

$-8.899041 \mathrm{e}-02$
$1.009032 \mathrm{e}-01$

$1.789268 \mathrm{e}-01$

$1.178507 \mathrm{e}-01$

4.199881e-02

$6.349886 \mathrm{e}-02$

$1.441714 \mathrm{e}-01$

$5.710307 \mathrm{e}-01$

$4.941445 \mathrm{e}-01$

$8.517044 \mathrm{e}-02$

$2.096840 \mathrm{e}-01$

$1.122629 \mathrm{e}-01$

$6.475690 \mathrm{e}-02$

4.194251e-02

$5.713239 \mathrm{e}-02$

$1.521050 \mathrm{e}-01$

$2.133056 \mathrm{e}-02$

$2.906953 \mathrm{e}-02$

$2.098412 \mathrm{e}-01$

9.615524e-02

$1.092037 \mathrm{e}-01$

$5.475452 \mathrm{e}-02$

$3.767048 \mathrm{e}-02$

$3.907047 \mathrm{e}-02$

$5.350468 \mathrm{e}-02$

$2.129644 \mathrm{e}-01$

5.915551e-02

$8.539080 \mathrm{e}-02$

$6.901388 \mathrm{e}-02$

$4.591912 \mathrm{e}-02$

$9.825206 \mathrm{e}-02$

4.430467e-02

$6.590010 \mathrm{e}-02$

6.766987e-02

2.182061e-02

$6.535501 \mathrm{e}-02$

$7.050194 \mathrm{e}-02$

$1.035649 \mathrm{e}-01$

9.048811e-02

$7.558648 \mathrm{e}-02$

$2.202806 \mathrm{e}-01$

$3.613843 \mathrm{e}-02$

$3.829938 \mathrm{e}-02$

$1.145056 \mathrm{e}-01$

2.614042e-01

$7.687855 \mathrm{e}-02$

$1.417301 \mathrm{e}-01$

$1.204674 \mathrm{e}-01$

$1.503236 \mathrm{e}-01$

$3.205052 \mathrm{e}-02$

$2.574450 \mathrm{e}-02$

$6.976798 \mathrm{e}-02$

$1.063329 \mathrm{e}-01$

$3.844242 \mathrm{e}-02$

$2.103111 \mathrm{e}-02$

$3.346537 \mathrm{e}-02$

$1.009149 \mathrm{e}-01$

$3.902893 \mathrm{e}-02$

$7.949750 \mathrm{e}-02$

$2.063930 \mathrm{e}-01$

$2.759736 \mathrm{e}-02$

$3.983728 \mathrm{e}-02$

$1.286051 \mathrm{e}-01$

$3.068963 \mathrm{e}-02$

$5.883574 \mathrm{e}-02$

$3.230613 \mathrm{e}-02$

$2.101685 \mathrm{e}-02$

8.114633e-02

$2.597399 \mathrm{e}-01$

$9.419055 \mathrm{e}-02$

$2.815969 \mathrm{e}-02$

$6.049234 \mathrm{e}-01$

$4.725021 \mathrm{e}-01$

$6.652752 \mathrm{e}-01$

$7.906552 \mathrm{e}-01$

$1.477112 \mathrm{e}-04$

$2.771889 \mathrm{e}-01$

$1.357175 \mathrm{e}-01$

$1.492727 \mathrm{e}-01$

$9.415697 \mathrm{e}-02$

$3.974932 \mathrm{e}-01$

$3.072199 \mathrm{e}-01$

7.618899e-01 


\begin{tabular}{|c|c|c|}
\hline 691 & Certain_Framing & Animal_science \\
\hline 692 & Certain_Framing & Nursing \\
\hline 693 & Certain_Framing & Seismology \\
\hline 694 & Certain_Framing & History \\
\hline 695 & Certain_Framing & Psychiatry \\
\hline 696 & Certain_Framing & Zoology \\
\hline 697 & Certain_Framing & Psychology \\
\hline 698 & Certain_Framing & Etiology \\
\hline 699 & Certain_Framing & Geomorphology \\
\hline 700 & Certain_Framing & Atmospheric_sciences \\
\hline 701 & Certain_Framing & Geography \\
\hline 702 & Certain_Framing & Demography \\
\hline 703 & Certain_Framing & Geology \\
\hline 704 & Certain_Framing & Medicine \\
\hline 705 & Certain_Framing & Family_medicine \\
\hline 706 & Certain_Framing & Evolutionary_biology \\
\hline 707 & Certain_Framing & Astrobiology \\
\hline 708 & Certain_Framing & Astronomy \\
\hline 709 & Certain_Framing & Astrophysics \\
\hline 710 & Certain_Framing & Geochemistry \\
\hline 711 & Certain_Framing & Business \\
\hline 712 & Certain_Framing & Finance \\
\hline 713 & Certain_Framing & Management_science \\
\hline 714 & Certain_Framing & Social_psychology \\
\hline 715 & Certain_Framing & Political_science \\
\hline 716 & Certain_Framing & Archaeology \\
\hline 717 & Certain_Framing & Economics \\
\hline 718 & Certain_Framing & Paleontology \\
\hline 719 & Certain_Framing & Criminology \\
\hline 720 & Uncertain_Number & Intercept \\
\hline 721 & Uncertain_Number & source[T.news] \\
\hline 722 & Uncertain_Number & finding_len \\
\hline 723 & Uncertain_Number & flesch_reading_ease \\
\hline 724 & Uncertain_Number & first_author_rank \\
\hline 725 & Uncertain_Number & affi_rank \\
\hline 726 & Uncertain_Number & num_authors \\
\hline 727 & Uncertain_Number & journal_impact \\
\hline 728 & Uncertain_Number & Law \\
\hline 729 & Uncertain_Number & Condensed_matter_physics \\
\hline 730 & Uncertain_Number & Biomedical_engineering \\
\hline 731 & Uncertain_Number & Hydrology \\
\hline 732 & Uncertain_Number & Atomic_physics \\
\hline 733 & Uncertain_Number & Development_economics \\
\hline 734 & Uncertain_Number & Optics \\
\hline 735 & Uncertain_Number & Public_policy \\
\hline 736 & Uncertain_Number & Lung_cancer \\
\hline 737 & Uncertain_Number & Electronic_engineering \\
\hline 738 & Uncertain_Number & Artificial_intelligence \\
\hline 739 & Uncertain_Number & Machine_learning \\
\hline 740 & Uncertain_Number & Gender_studies \\
\hline 741 & Uncertain_Number & Chemical_engineering \\
\hline 742 & Uncertain_Number & Biophysics \\
\hline 743 & Uncertain_Number & Analytical_chemistry \\
\hline 744 & Uncertain_Number & Computer_science \\
\hline 745 & Uncertain_Number & Radiology \\
\hline 746 & Uncertain_Number & Urology \\
\hline 747 & Uncertain_Number & Architecture \\
\hline 748 & Uncertain_Number & Quantum_mechanics \\
\hline 749 & Uncertain_Number & Particle_physics \\
\hline 750 & Uncertain_Number & Materials_science \\
\hline 751 & Uncertain_Number & Computational_biology \\
\hline 752 & Uncertain_Number & Agronomy \\
\hline 753 & Uncertain_Number & $\begin{array}{l}\text { Environmental_resource man- } \\
\text { agement }\end{array}$ \\
\hline 754 & Uncertain_Number & Molecular_biology \\
\hline 755 & Uncertain_Number & Dermatology \\
\hline 756 & Uncertain_Number & Engineering \\
\hline 757 & Uncertain_Number & Surgery \\
\hline 758 & Uncertain_Number & Public_health \\
\hline 759 & Uncertain_Number & Molecular_physics \\
\hline
\end{tabular}

$\begin{array}{rr}-5.872492 \mathrm{e}-03 & 1.471485 \mathrm{e}-01 \\ 8.471809 \mathrm{e}-02 & 3.817098 \mathrm{e}-02 \\ -7.971247 \mathrm{e}-02 & 1.614144 \mathrm{e}-01 \\ -3.112217 \mathrm{e}-01 & 4.516456 \mathrm{e}-01 \\ -5.876560 \mathrm{e}-02 & 2.675840 \mathrm{e}-02 \\ 1.314946 \mathrm{e}-01 & 7.017966 \mathrm{e}-02 \\ -1.012912 \mathrm{e}-01 & 5.838622 \mathrm{e}-02 \\ 3.149850 \mathrm{e}-01 & 7.485358 \mathrm{e}-02 \\ -3.309416 \mathrm{e}-01 & 8.805330 \mathrm{e}-02\end{array}$

$\begin{array}{rr}-3.309416 \mathrm{e}-01 & 8.805330 \mathrm{e}-02 \\ 3.980629 \mathrm{e}-01 & 9.447900 \mathrm{e}-02\end{array}$

$8.155008 \mathrm{e}-03 \quad 8.120337 \mathrm{e}-02$

$-5.086070 \mathrm{e}-02 \quad 2.467391 \mathrm{e}-02$

$1.404526 \mathrm{e}-01 \quad 8.828025 \mathrm{e}-02$

$-1.125198 \mathrm{e}-01 \quad 5.030955 \mathrm{e}-02$

$4.556540 \mathrm{e}-02 \quad 2.515277 \mathrm{e}-02$

$2.341686 \mathrm{e}-01 \quad 6.273572 \mathrm{e}-02$

$-2.823186 \mathrm{e}-01 \quad 1.335766 \mathrm{e}-01$

$1.473447 \mathrm{e}-01 \quad 1.002120 \mathrm{e}-01$

$3.608433 \mathrm{e}-01 \quad 1.545841 \mathrm{e}-01$

$-2.768964 \mathrm{e}-03 \quad 1.500584 \mathrm{e}-01$

$-9.469593 \mathrm{e}-02 \quad 8.568585 \mathrm{e}-02$

$1.294520 \mathrm{e}-01 \quad 9.077063 \mathrm{e}-02$

$-4.501820 \mathrm{e}-12 \quad 1.455190 \mathrm{e}-12$

9.043926e-02 2.938271e-02

$-2.341919 \mathrm{e}-01 \quad 1.365079 \mathrm{e}-01$

3.077281e-01 $1.327574 \mathrm{e}-01$

$-3.336342 \mathrm{e}-02 \quad 6.705884 \mathrm{e}-02$

$-1.203383 \mathrm{e}-01 \quad 8.213819 \mathrm{e}-02$

$-2.083024 \mathrm{e}-01 \quad 1.912835 \mathrm{e}-01$

$8.036772 \mathrm{e}-02 \quad 2.829587 \mathrm{e}-02$

$9.854131 \mathrm{e}-03 \quad 3.975506 \mathrm{e}-03$

$2.530194 \mathrm{e}-03 \quad 2.243658 \mathrm{e}-04$

9.689563e-04 8.949156e-05

$-4.109076 \mathrm{e}-06 \quad 1.145942 \mathrm{e}-06$

$-4.135660 \mathrm{e}-06 \quad 1.142885 \mathrm{e}-06$

$1.594579 \mathrm{e}-04 \quad 1.558144 \mathrm{e}-04$

$4.312048 \mathrm{e}-05 \quad 1.606494 \mathrm{e}-04$

$1.876525 \mathrm{e}-01 \quad 7.792099 \mathrm{e}-02$

$3.462799 \mathrm{e}-01 \quad 1.587575 \mathrm{e}-01$

$-3.719016 \mathrm{e}-01 \quad 1.499879 \mathrm{e}-01$

$1.926085 \mathrm{e}-01 \quad 1.315513 \mathrm{e}-01$

$-1.662291 \mathrm{e}-01 \quad 1.867234 \mathrm{e}-01$

$2.104808 \mathrm{e}-01 \quad 2.124863 \mathrm{e}-01$

$-7.513547 \mathrm{e}-01 \quad 2.318179 \mathrm{e}-01$

$-1.975167 \mathrm{e}-01 \quad 1.426255 \mathrm{e}-01$

$8.149596 \mathrm{e}-02 \quad 3.385900 \mathrm{e}-02$

$5.978822 \mathrm{e}-01 \quad 3.895987 \mathrm{e}-01$

$-2.143497 \mathrm{e}-01 \quad 1.970069 \mathrm{e}-01$

$-9.886740 \mathrm{e}-02 \quad 2.086902 \mathrm{e}-01$

$8.717666 \mathrm{e}-02 \quad 1.254682 \mathrm{e}-01$

$-5.415848 \mathrm{e}-01 \quad 1.464673 \mathrm{e}-01$

$3.179963 \mathrm{e}-01 \quad 8.986158 \mathrm{e}-02$

$2.313544 \mathrm{e}-01 \quad 1.593473 \mathrm{e}-01$

$3.218781 \mathrm{e}-01 \quad 1.049546 \mathrm{e}-01$

$-5.179288 \mathrm{e}-02 \quad 3.740298 \mathrm{e}-02$

$-9.312205 \mathrm{e}-02 \quad 5.655033 \mathrm{e}-02$

$-7.487101 \mathrm{e}-02 \quad 1.283950 \mathrm{e}-01$

$-2.311866 \mathrm{e}-01 \quad 5.085442 \mathrm{e}-01$

$-4.852988 \mathrm{e}-01 \quad 4.400715 \mathrm{e}-01$

$5.384376 \mathrm{e}-01 \quad 7.585045 \mathrm{e}-02$

$-2.063257 \mathrm{e}-01 \quad 1.867387 \mathrm{e}-01$

$2.292139 \mathrm{e}-02 \quad 9.997824 \mathrm{e}-02$

$1.271473 \mathrm{e}-01 \quad 5.767070 \mathrm{e}-02$

$-4.115803 \mathrm{e}-02$

$-2.519845 \mathrm{e}-02$

$-1.548033 \mathrm{e}-01$

$-2.560537 \mathrm{e}-02$

$1.709295 \mathrm{e}-01$

$-2.711366 \mathrm{e}-01$ 9.681666e-01

$2.647414 \mathrm{e}-02$

$6.214292 \mathrm{e}-01$

$4.907827 \mathrm{e}-01$

$2.809837 \mathrm{e}-02$

$6.099606 \mathrm{e}-02$

$8.279123 \mathrm{e}-02$

$2.593465 \mathrm{e}-05$

$1.717327 \mathrm{e}-04$

$2.534220 \mathrm{e}-05$

9.200069e-01

$3.929275 \mathrm{e}-02$

$1.116370 \mathrm{e}-01$

$2.533260 \mathrm{e}-02$

$7.007938 \mathrm{e}-02$

$1.903030 \mathrm{e}-04$

$3.457410 \mathrm{e}-02$

$1.414965 \mathrm{e}-01$

$1.959593 \mathrm{e}-02$

9.852781e-01

$2.691139 \mathrm{e}-01$

$1.538507 \mathrm{e}-01$

$1.981411 \mathrm{e}-03$

$2.088442 \mathrm{e}-03$

$8.626046 \mathrm{e}-02$

$2.046617 \mathrm{e}-02$

$6.188275 \mathrm{e}-01$

$1.429256 \mathrm{e}-01$

$2.761865 \mathrm{e}-01$

$4.514603 \mathrm{e}-03$

$1.319834 \mathrm{e}-02$

2.327746e-29

$3.333206 \mathrm{e}-27$

$3.373219 \mathrm{e}-04$

2.973018e-04

$3.061454 \mathrm{e}-01$

$7.883853 \mathrm{e}-01$

$1.604339 \mathrm{e}-02$

$2.918736 \mathrm{e}-02$

$1.316755 \mathrm{e}-02$

$1.431819 \mathrm{e}-01$

$3.733522 \mathrm{e}-01$

$3.219179 \mathrm{e}-01$

$1.193506 \mathrm{e}-03$

$1.661180 \mathrm{e}-01$

$1.610142 \mathrm{e}-02$

$1.249038 \mathrm{e}-01$

$2.766013 \mathrm{e}-01$

$6.356846 \mathrm{e}-01$

$4.871863 \mathrm{e}-01$

$2.184954 \mathrm{e}-04$

$4.034473 \mathrm{e}-04$

$1.465567 \mathrm{e}-01$

$2.167830 \mathrm{e}-03$

$1.661599 \mathrm{e}-01$

$9.964155 \mathrm{e}-02$

$5.598158 \mathrm{e}-01$

6.494011e-01

$2.701463 \mathrm{e}-01$

$1.324733 \mathrm{e}-12$

2.692278e-01

$8.186675 \mathrm{e}-01$

2.749167e-02

$2.705379 \mathrm{e}-01$

$6.204339 \mathrm{e}-01$

$2.531457 \mathrm{e}-01$

$1.777120 \mathrm{e}-01$

$4.198612 \mathrm{e}-11$

$1.468405 \mathrm{e}-01$
$*$

* 
760 Uncertain_Number

761 Uncertain_Number

762 Uncertain_Number

763 Uncertain_Number

764 Uncertain_Number

765 Uncertain_Number

766 Uncertain_Number

767 Uncertain_Number

768 Uncertain_Number

769 Uncertain_Number

770 Uncertain_Number

771 Uncertain_Number

772 Uncertain_Number

773 Uncertain_Number

774 Uncertain_Number

775 Uncertain_Number

776 Uncertain_Number

777 Uncertain_Number

778 Uncertain_Number

779 Uncertain_Number

780 Uncertain_Number

781 Uncertain_Number

782 Uncertain_Number

783 Uncertain_Number

784 Uncertain_Number

785 Uncertain_Number

786 Uncertain_Number

787 Uncertain_Number

788 Uncertain_Number

789 Uncertain_Number

790 Uncertain_Number

791 Uncertain_Number

792 Uncertain_Number

793 Uncertain_Number

794 Uncertain_Number

795 Uncertain_Number

796 Uncertain_Number

797 Uncertain_Number

798 Uncertain_Number

799 Uncertain_Number

800 Uncertain_Number

801 Uncertain_Number

802 Uncertain_Number

803 Uncertain_Number

804 Uncertain_Number

805 Uncertain_Number

806 Uncertain_Number

807 Uncertain_Number

808 Uncertain_Number

809 Uncertain_Number

810 Uncertain_Number

811 Uncertain_Number

812 Uncertain_Number

813 Uncertain_Number

814 Uncertain_Number

815 Uncertain_Number

816 Uncertain_Number

817 Uncertain_Number

818 Uncertain_Number

819 Uncertain_Number

820 Uncertain_Number

821 Uncertain_Number

822 Uncertain_Number

823 Uncertain_Number

824 Uncertain_Number

825 Uncertain_Number

826 Uncertain_Number

827 Uncertain_Number

828 Uncertain_Number

829 Uncertain_Number
Bioinformatics

Physics

Cognitive_psychology

Oncology

Pathology

Biochemistry

Social_science

Climatology

Pharmacology

Agriculture

Neuroscience

Fishery

Virology

Microbiology

Communication

Endocrinology

Epigenetics

Marketing

Global_health

Agroforestry

Chemistry

Mathematics

Gynecology

Gerontology

Meteorology

Veterinary_medicine

Government

Dentistry

Microeconomics

Sociology

Emergency_medicine

Pediatrics

Advertising

Oceanography

Developmental_psychology

Internal_medicine

Cardiology

Environmental_engineering

General_surgery

Environmental_science

Geophysics

Immunology

Clinical_psychology

Toxicology

Ecology

Biology

Epidemiology

Physical_therapy

Physiology

Statistics

Psychopathology

Animal_science

Nursing

Seismology

History

Psychiatry

Zoology

Psychology

Etiology

Geomorphology

Atmospheric_sciences

Geography

Demography

Geology

Medicine

Family_medicine

Evolutionary_biology

Astrobiology

Astronomy

Astrophysics 2.669767e-02

$5.213570 \mathrm{e}-01$

$-2.394485 \mathrm{e}-02$

$-6.487146 \mathrm{e}-02$

$-1.926027 \mathrm{e}-02$

$4.004910 \mathrm{e}-02$

$8.343142 \mathrm{e}-02$

$-9.674010 \mathrm{e}-02$

$-1.025468 \mathrm{e}-01$

$1.764185 \mathrm{e}-01$

$-8.918402 \mathrm{e}-02$

$-2.097097 \mathrm{e}-01$

$1.018581 \mathrm{e}-01$

$-1.283548 \mathrm{e}-01$

$-1.183637 \mathrm{e}-01$

$-2.252941 \mathrm{e}-02$

$-8.449373 \mathrm{e}-02$

$-6.220513 \mathrm{e}-03$

$-7.477826 \mathrm{e}-02$

$5.294632 \mathrm{e}-02$

$-1.773415 \mathrm{e}-02$

$-1.391172 \mathrm{e}-01$

$9.496896 \mathrm{e}-02$

$-1.420052 \mathrm{e}-02$

$-2.228219 \mathrm{e}-02$

$5.301580 \mathrm{e}-01$

$-6.932230 \mathrm{e}-02$

$-1.779317 \mathrm{e}-01$

$1.177684 \mathrm{e}-01$

$-1.393366 \mathrm{e}-01$

$4.088170 \mathrm{e}-02$

$-3.070933 \mathrm{e}-02$

$-2.440927 \mathrm{e}-02$

$-1.913469 \mathrm{e}-01$

$-3.678099 \mathrm{e}-02$

$-6.386282 \mathrm{e}-02$

$-5.415164 \mathrm{e}-02$

$-1.898473 \mathrm{e}-01$

$-1.767917 \mathrm{e}-02$

$3.948327 \mathrm{e}-02$

$-2.312014 \mathrm{e}-01$

$-5.781182 \mathrm{e}-02$

$-6.816074 \mathrm{e}-02$

$2.076576 \mathrm{e}-01$

$2.738174 \mathrm{e}-02$

$-4.102415 \mathrm{e}-02$

$1.176510 \mathrm{e}-01$

$-2.958667 \mathrm{e}-02$

$-7.205563 \mathrm{e}-02$

$9.034200 \mathrm{e}-02$

$-1.024174 \mathrm{e}-02$

$-8.864893 \mathrm{e}-02$

$-3.223246 \mathrm{e}-02$

$-3.794824 \mathrm{e}-01$

$-6.974666 \mathrm{e}-02$

$3.308198 \mathrm{e}-02$

$-9.734051 \mathrm{e}-02$

$-5.354672 \mathrm{e}-02$

$-1.112802 \mathrm{e}-01$

$-5.311842 \mathrm{e}-02$

$-8.300485 \mathrm{e}-02$

$5.294004 \mathrm{e}-02$

$3.760599 \mathrm{e}-03$

$3.327749 \mathrm{e}-01$

$-8.343590 \mathrm{e}-02$

$6.064260 \mathrm{e}-02$

2.494916e-01

$-8.505536 \mathrm{e}-02$

$1.408712 \mathrm{e}-01$

$-3.037560 \mathrm{e}-02$
$8.563320 \mathrm{e}-02$

$9.725384 \mathrm{e}-02$

4.876286e-02

$3.354829 \mathrm{e}-02$

$3.479508 \mathrm{e}-02$

$4.764979 \mathrm{e}-02$

$1.896602 \mathrm{e}-01$

$5.268226 \mathrm{e}-02$

$7.604669 \mathrm{e}-02$

$6.146185 \mathrm{e}-02$

4.089430e-02

$8.750057 \mathrm{e}-02$

$3.945652 \mathrm{e}-02$

$5.868881 \mathrm{e}-02$

6.026491e-02

$1.943283 \mathrm{e}-02$

$5.820337 \mathrm{e}-02$

$6.278709 \mathrm{e}-02$

$9.223205 \mathrm{e}-02$

$8.058621 \mathrm{e}-02$

$6.731524 \mathrm{e}-02$

$1.961758 \mathrm{e}-01$

$3.218388 \mathrm{e}-02$

$3.410837 \mathrm{e}-02$

$1.019756 \mathrm{e}-01$

$2.327994 \mathrm{e}-01$

6.846592e-02

$1.262210 \mathrm{e}-01$

$1.072849 \mathrm{e}-01$

$1.338740 \mathrm{e}-01$

$2.854331 \mathrm{e}-02$

$2.292735 \mathrm{e}-02$

$6.213344 \mathrm{e}-02$

$9.469719 \mathrm{e}-02$

$3.423576 \mathrm{e}-02$

$1.872973 \mathrm{e}-02$

$2.980334 \mathrm{e}-02$

8.987206e-02

$3.475808 \mathrm{e}-02$

$7.079828 \mathrm{e}-02$

$1.838079 \mathrm{e}-01$

$2.457745 \mathrm{e}-02$

$3.547798 \mathrm{e}-02$

$1.145322 \mathrm{e}-01$

$2.733134 \mathrm{e}-02$

$5.239749 \mathrm{e}-02$

$2.877094 \mathrm{e}-02$

$1.871703 \mathrm{e}-02$

$7.226668 \mathrm{e}-02$

$2.313172 \mathrm{e}-01$

$8.388350 \mathrm{e}-02$

$1.310464 \mathrm{e}-01$

3.399403e-02

$1.437512 \mathrm{e}-01$

$4.022231 \mathrm{e}-01$

$2.383029 \mathrm{e}-02$

$6.250006 \mathrm{e}-02$

$5.199715 \mathrm{e}-02$

$6.666253 \mathrm{e}-02$

$7.841784 \mathrm{e}-02$

$8.414039 \mathrm{e}-02$

$7.231748 \mathrm{e}-02$

$2.197390 \mathrm{e}-02$

$7.861996 \mathrm{e}-02$

$4.480430 \mathrm{e}-02$

$2.240036 \mathrm{e}-02$

$5.587070 \mathrm{e}-02$

$1.189596 \mathrm{e}-01$

8.924603e-02

$1.376684 \mathrm{e}-01$

7.552220e-01

$8.427141 \mathrm{e}-08$

$6.234015 \mathrm{e}-01$

$5.317477 \mathrm{e}-02$

$5.799071 \mathrm{e}-01$

$4.006500 \mathrm{e}-01$

$6.600173 \mathrm{e}-01$

$6.633698 \mathrm{e}-02$

$1.775302 \mathrm{e}-01$

$4.106454 \mathrm{e}-03$ 
830 Uncertain_Number

831 Uncertain_Number

832 Uncertain_Number

833 Uncertain_Number

834 Uncertain_Number

835 Uncertain_Number

836 Uncertain_Number

837 Uncertain_Number

838 Uncertain_Number

839 Uncertain_Number

840 Uncertain_Extent

841 Uncertain_Extent

842 Uncertain_Extent

843 Uncertain_Extent

844 Uncertain_Extent

845 Uncertain_Extent

846 Uncertain_Extent

847 Uncertain_Extent

848 Uncertain_Extent

849 Uncertain_Extent

850 Uncertain_Extent

851 Uncertain_Extent

852 Uncertain_Extent

853 Uncertain_Extent

854 Uncertain_Extent

855 Uncertain_Extent

856 Uncertain_Extent

857 Uncertain_Extent

858 Uncertain_Extent

859 Uncertain_Extent

860 Uncertain_Extent

861 Uncertain_Extent

862 Uncertain_Extent

863 Uncertain_Extent

864 Uncertain_Extent

865 Uncertain_Extent

866 Uncertain_Extent

867 Uncertain_Extent

868 Uncertain_Extent

869 Uncertain_Extent

870 Uncertain_Extent

871 Uncertain_Extent

872 Uncertain_Extent

873 Uncertain_Extent

874 Uncertain_Extent

875 Uncertain_Extent

876 Uncertain_Extent

877 Uncertain_Extent

878 Uncertain_Extent

879 Uncertain_Extent

880 Uncertain_Extent

881 Uncertain_Extent

882 Uncertain_Extent

883 Uncertain_Extent

884 Uncertain_Extent

885 Uncertain_Extent

886 Uncertain_Extent

887 Uncertain_Extent

888 Uncertain_Extent

889 Uncertain_Extent

890 Uncertain_Extent

891 Uncertain_Extent

892 Uncertain_Extent

893 Uncertain_Extent

894 Uncertain_Extent

895 Uncertain_Extent

896 Uncertain_Extent

897 Uncertain_Extent

898 Uncertain_Extent
Geochemistry

Business

Finance

Management_science

Social_psychology

Political_science

Archaeology

Economics

Paleontology

Criminology

Intercept

source[T.news]

finding_len

flesch_reading_ease

first_author_rank

affi_rank

num_authors

journal_impact

Law

Condensed_matter_physics

Biomedical_engineering

Hydrology

Atomic_physics

Development_economics

Optics

Public_policy

Lung_cancer

Electronic_engineering

Artificial_intelligence

Machine_learning

Gender_studies

Chemical_engineering

Biophysics

Analytical_chemistry

Computer_science

Radiology

Urology

Architecture

Quantum_mechanics

Particle_physics

Materials_science

Computational_biology

Agronomy

Environmental_resource management

Molecular_biology

Dermatology

Engineering

Surgery

Public health

Molecular_physics

Bioinformatics

Physics

Cognitive_psychology

Oncology

Pathology

Biochemistry

Social_science

Climatology

Pharmacology

Agriculture

Neuroscience

Fishery

Virology

Microbiology

Communication

Endocrinology

Epigenetics

Marketing

Global health 8.971024e-02

$-5.698424 \mathrm{e}-02$

$-3.738821 \mathrm{e}-02$

$-2.165602 \mathrm{e}-12$

$-6.725829 \mathrm{e}-02$

$3.469279 \mathrm{e}-01$

$1.174663 \mathrm{e}-01$

$1.005533 \mathrm{e}-01$

$1.346898 \mathrm{e}-01$

$-1.528824 \mathrm{e}-01$

$1.384817 \mathrm{e}-01$

$1.141141 \mathrm{e}-02$

$1.697607 \mathrm{e}-03$

$5.019145 \mathrm{e}-04$

$-2.633616 \mathrm{e}-06$

$-3.149592 \mathrm{e}-06$

$-6.797947 \mathrm{e}-04$

$4.552613 \mathrm{e}-04$

$3.601845 \mathrm{e}-02$

$2.039316 \mathrm{e}-01$

$-1.945339 \mathrm{e}-01$

$-1.382001 \mathrm{e}-01$

$-2.374851 \mathrm{e}-01$

$1.204365 \mathrm{e}+00$

$-6.632538 \mathrm{e}-03$

$-2.716704 \mathrm{e}-01$

$-9.329557 \mathrm{e}-03$

$-6.732345 \mathrm{e}-01$

$-4.193149 \mathrm{e}-01$

7.885666e-02

$3.296619 \mathrm{e}-01$

$-5.681293 \mathrm{e}-01$

$1.544716 \mathrm{e}-01$

$-6.773311 \mathrm{e}-02$

$3.239349 \mathrm{e}-01$

$3.714296 \mathrm{e}-02$

$-1.040627 \mathrm{e}-01$

$-2.395985 \mathrm{e}-01$

$-3.159762 \mathrm{e}-01$

$-2.317123 \mathrm{e}-01$

$4.261880 \mathrm{e}-01$

$-3.219202 \mathrm{e}-01$

$2.218747 \mathrm{e}-01$

1.267310e-01

$-3.655988 \mathrm{e}-02$

$-6.431341 \mathrm{e}-02$

$-1.518751 \mathrm{e}-01$

$-7.811676 \mathrm{e}-02$

$6.465609 \mathrm{e}-02$

$-2.779015 \mathrm{e}-01$

$4.519419 \mathrm{e}-02$

$2.684745 \mathrm{e}-01$

$3.173258 \mathrm{e}-02$

$-7.714572 \mathrm{e}-02$

$1.118740 \mathrm{e}-01$

$-3.557743 \mathrm{e}-02$

$1.477850 \mathrm{e}-01$

$-6.463593 \mathrm{e}-02$

$-1.156023 \mathrm{e}-01$

$2.811641 \mathrm{e}-01$

$-4.877828 \mathrm{e}-02$

$3.749330 \mathrm{e}-01$

-1.370250e-01

$-5.990317 \mathrm{e}-02$

$-1.855242 \mathrm{e}-01$

$-2.151911 \mathrm{e}-02$

$-7.483090 \mathrm{e}-02$

$-8.654938 \mathrm{e}-02$

4.105602e-01
$1.336379 \mathrm{e}-01$

$7.630945 \mathrm{e}-02$

$8.083782 \mathrm{e}-02$

$1.295952 \mathrm{e}-12$

$2.616743 \mathrm{e}-02$

$1.215702 \mathrm{e}-01$

$1.182301 \mathrm{e}-01$

$5.972075 \mathrm{e}-02$

$7.315000 \mathrm{e}-02$

$1.703518 \mathrm{e}-01$

$3.966968 \mathrm{e}-02$

$5.573500 \mathrm{e}-03$

$3.145519 \mathrm{e}-04$

$1.254636 \mathrm{e}-04$

$1.606565 \mathrm{e}-06$

$1.602279 \mathrm{e}-06$

$2.184455 \mathrm{e}-04$

$2.252241 \mathrm{e}-04$

$1.092421 \mathrm{e}-01$

$2.225717 \mathrm{e}-01$

2.102770e-01

$1.844297 \mathrm{e}-01$

$2.617788 \mathrm{e}-01$

$2.978973 \mathrm{e}-01$

$3.249995 \mathrm{e}-01$

$1.999552 \mathrm{e}-01$

4.746897e-02

$5.462019 \mathrm{e}-01$

$2.761958 \mathrm{e}-01$

$2.925753 \mathrm{e}-01$

$1.759014 \mathrm{e}-01$

$2.053412 \mathrm{e}-01$

$1.259823 \mathrm{e}-01$

$2.233985 \mathrm{e}-01$

$1.471421 \mathrm{e}-01$

$5.243748 \mathrm{e}-02$

7.928131e-02

$1.800047 \mathrm{e}-01$

$7.129587 \mathrm{e}-01$

6.169627e-01

$1.063393 \mathrm{e}-01$

$2.618003 \mathrm{e}-01$

$1.401655 \mathrm{e}-01$

$8.085203 \mathrm{e}-02$

$5.236720 \mathrm{e}-02$

7.133247e-02

$1.899103 \mathrm{e}-01$

$2.663220 \mathrm{e}-02$

$3.629467 \mathrm{e}-02$

$2.619966 \mathrm{e}-01$

$1.200543 \mathrm{e}-01$

$1.363460 \mathrm{e}-01$

$6.836359 \mathrm{e}-02$

$4.703336 \mathrm{e}-02$

4.878131e-02

$6.680311 \mathrm{e}-02$

$2.658961 \mathrm{e}-01$

$7.385843 \mathrm{e}-02$

$1.066144 \mathrm{e}-01$

8.616707e-02

$5.733218 \mathrm{e}-02$

$1.226723 \mathrm{e}-01$

$5.531646 \mathrm{e}-02$

8.227937e-02

$8.448901 \mathrm{e}-02$

$2.724406 \mathrm{e}-02$

$8.159880 \mathrm{e}-02$

$8.802499 \mathrm{e}-02$

$1.293056 \mathrm{e}-01$

5.020455e-01

$4.552267 \mathrm{e}-01$

$6.437241 \mathrm{e}-01$

$9.473557 \mathrm{e}-02$

$1.017188 \mathrm{e}-02$

$4.327793 \mathrm{e}-03$

3.204654e-01

$9.225877 \mathrm{e}-02$

$6.560295 \mathrm{e}-02$

$3.694950 \mathrm{e}-01$

4.830408e-04

$4.063482 \mathrm{e}-02$

$6.898547 \mathrm{e}-08$ 


\begin{tabular}{|c|c|c|}
\hline 899 & Uncertain_Extent & Agroforestry \\
\hline 900 & Uncertain_Extent & Chemistry \\
\hline 901 & Uncertain_Extent & Mathematics \\
\hline 902 & Uncertain_Extent & Gynecology \\
\hline 903 & Uncertain_Extent & Gerontology \\
\hline 904 & Uncertain_Extent & Meteorology \\
\hline 905 & Uncertain_Extent & Veterinary_medicine \\
\hline 906 & Uncertain_Extent & Government \\
\hline 907 & Uncertain_Extent & Dentistry \\
\hline 908 & Uncertain_Extent & Microeconomics \\
\hline 909 & Uncertain_Extent & Sociology \\
\hline 910 & Uncertain_Extent & Emergency_medicine \\
\hline 911 & Uncertain_Extent & Pediatrics \\
\hline 912 & Uncertain_Extent & Advertising \\
\hline 913 & Uncertain_Extent & Oceanography \\
\hline 914 & Uncertain_Extent & Developmental_psychology \\
\hline 915 & Uncertain_Extent & Internal_medicine \\
\hline 916 & Uncertain_Extent & Cardiology \\
\hline 917 & Uncertain_Extent & Environmental_engineering \\
\hline 918 & Uncertain_Extent & General_surgery \\
\hline 919 & Uncertain_Extent & Environmental_science \\
\hline 920 & Uncertain_Extent & Geophysics \\
\hline 921 & Uncertain_Extent & Immunology \\
\hline 922 & Uncertain_Extent & Clinical_psychology \\
\hline 923 & Uncertain_Extent & Toxicology \\
\hline 924 & Uncertain_Extent & Ecology \\
\hline 925 & Uncertain_Extent & Biology \\
\hline 926 & Uncertain_Extent & Epidemiology \\
\hline 927 & Uncertain_Extent & Physical_therapy \\
\hline 928 & Uncertain_Extent & Physiology \\
\hline 929 & Uncertain_Extent & Statistics \\
\hline 930 & Uncertain_Extent & Psychopathology \\
\hline 931 & Uncertain_Extent & Animal_science \\
\hline 932 & Uncertain_Extent & Nursing \\
\hline 933 & Uncertain_Extent & Seismology \\
\hline 934 & Uncertain_Extent & History \\
\hline 935 & Uncertain_Extent & Psychiatry \\
\hline 936 & Uncertain_Extent & Zoology \\
\hline 937 & Uncertain_Extent & Psychology \\
\hline 938 & Uncertain_Extent & Etiology \\
\hline 939 & Uncertain_Extent & Geomorphology \\
\hline 940 & Uncertain_Extent & Atmospheric_sciences \\
\hline 941 & Uncertain_Extent & Geography \\
\hline 942 & Uncertain_Extent & Demography \\
\hline 943 & Uncertain_Extent & Geology \\
\hline 944 & Uncertain_Extent & Medicine \\
\hline 945 & Uncertain_Extent & Family_medicine \\
\hline 946 & Uncertain_Extent & Evolutionary_biology \\
\hline 947 & Uncertain_Extent & Astrobiology \\
\hline 948 & Uncertain_Extent & Astronomy \\
\hline 949 & Uncertain_Extent & Astrophysics \\
\hline 950 & Uncertain_Extent & Geochemistry \\
\hline 951 & Uncertain_Extent & Business \\
\hline 952 & Uncertain_Extent & Finance \\
\hline 953 & Uncertain_Extent & Management_science \\
\hline 954 & Uncertain_Extent & Social_psychology \\
\hline 955 & Uncertain_Extent & Political_science \\
\hline 956 & Uncertain_Extent & Archaeology \\
\hline 957 & Uncertain_Extent & Economics \\
\hline 958 & Uncertain_Extent & Paleontology \\
\hline 959 & Uncertain_Extent & Criminology \\
\hline 960 & \multicolumn{2}{|c|}{ Uncertain_Probability Intercept } \\
\hline 961 & \multicolumn{2}{|c|}{ Uncertain_Probability source[T.news] } \\
\hline 962 & \multicolumn{2}{|c|}{ Uncertain_Probability finding_len } \\
\hline 963 & \multicolumn{2}{|c|}{ Uncertain_Probability flesch_reading_ease } \\
\hline 964 & \multicolumn{2}{|c|}{ Uncertain_Probability first_author_rank } \\
\hline 965 & \multicolumn{2}{|c|}{ Uncertain_Probability affi_rank } \\
\hline 966 & \multicolumn{2}{|c|}{ Uncertain_Probability num_authors } \\
\hline 967 & \multicolumn{2}{|c|}{ Uncertain_Probability journal_impact } \\
\hline 968 & \multicolumn{2}{|c|}{ Uncertain_Probability Law } \\
\hline
\end{tabular}

$-3.682025 \mathrm{e}-01$ 6.746584e-02 $-1.432857 \mathrm{e}-01$ $5.034229 \mathrm{e}-02$ $1.499427 \mathrm{e}-01$ $1.204120 \mathrm{e}-01$ $1.224024 \mathrm{e}+00$ $2.624025 \mathrm{e}-02$

$7.666300 \mathrm{e}-02$

$8.897237 \mathrm{e}-02$

$-2.394590 \mathrm{e}-01$

$6.182389 \mathrm{e}-02$

$1.178138 \mathrm{e}-02$

$1.087239 \mathrm{e}-01$

$1.542290 \mathrm{e}-01$

$6.605211 \mathrm{e}-02$

$-1.579629 \mathrm{e}-02$

$-8.207534 \mathrm{e}-02$

$4.434649 \mathrm{e}-01$

6.676467e-02

$3.124797 \mathrm{e}-01$

$4.970344 \mathrm{e}-01$

$1.565549 \mathrm{e}-02$

$-3.296156 \mathrm{e}-03$

$4.966148 \mathrm{e}-02$

$8.008658 \mathrm{e}-02$

$-3.127617 \mathrm{e}-02$

$5.069813 \mathrm{e}-02$

$-2.668499 \mathrm{e}-03$

$-1.775297 \mathrm{e}-01$

3.702986e-01

$1.490344 \mathrm{e}-01$

$-8.820164 \mathrm{e}-02$

$-3.328058 \mathrm{e}-02$

$-3.179278 \mathrm{e}-01$

$-2.972329 \mathrm{e}-01$

$8.243814 \mathrm{e}-02$

$1.206202 \mathrm{e}-02$

$-1.525205 \mathrm{e}-01$

$-4.481506 \mathrm{e}-02$

$1.024977 \mathrm{e}-01$

4.177647e-01

4.139853e-01

$-2.176771 \mathrm{e}-02$

3.065456e-01

$-9.027842 \mathrm{e}-02$

$1.576642 \mathrm{e}-01$

$-3.592217 \mathrm{e}-02$

$-5.446410 \mathrm{e}-01$

$-2.564328 \mathrm{e}-01$

$-7.217501 \mathrm{e}-03$

$2.512905 \mathrm{e}-01$

$1.052992 \mathrm{e}-01$

$2.695091 \mathrm{e}-02$

$-3.202171 \mathrm{e}-12$

$7.715509 \mathrm{e}-03$

$1.902772 \mathrm{e}-01$

$1.194541 \mathrm{e}-01$

$6.928585 \mathrm{e}-02$

$-1.833181 \mathrm{e}-02$

$4.582493 \mathrm{e}-01$

$1.859137 \mathrm{e}-01$

$1.495880 \mathrm{e}-02$

4.568004e-04

$-3.695115 \mathrm{e}-04$

$-3.524256 \mathrm{e}-06$

$2.824612 \mathrm{e}-06$

$-1.770833 \mathrm{e}-04$

$-1.030409 \mathrm{e}-03$

$2.175750 \mathrm{e}-01$ 1.129786e-01

$9.437328 \mathrm{e}-02$

2.750306e-01

$4.512052 \mathrm{e}-02$

$4.781858 \mathrm{e}-02$

$1.429657 \mathrm{e}-01$

$3.263755 \mathrm{e}-01$

$9.598649 \mathrm{e}-02$

$1.769567 \mathrm{e}-01$

$1.504092 \mathrm{e}-01$

$1.876860 \mathrm{e}-01$

$4.001658 \mathrm{e}-02$

$3.214322 \mathrm{e}-02$

$8.710860 \mathrm{e}-02$

$1.327617 \mathrm{e}-01$

$4.799717 \mathrm{e}-02$

$2.625833 \mathrm{e}-02$

$4.178309 \mathrm{e}-02$

$1.259970 \mathrm{e}-01$

$4.872945 \mathrm{e}-02$

$9.925636 \mathrm{e}-02$

$2.576914 \mathrm{e}-01$

$3.445660 \mathrm{e}-02$

$4.973872 \mathrm{e}-02$

$1.605695 \mathrm{e}-01$

$3.831745 \mathrm{e}-02$

$7.345919 \mathrm{e}-02$

$4.033572 \mathrm{e}-02$

$2.624053 \mathrm{e}-02$

$1.013150 \mathrm{e}-01$

$3.242975 \mathrm{e}-01$

$1.176013 \mathrm{e}-01$

$1.837218 \mathrm{e}-01$

4.765827e-02

$2.015334 \mathrm{e}-01$

$5.639007 \mathrm{e}-01$

$3.340912 \mathrm{e}-02$

$8.762260 \mathrm{e}-02$

$7.289794 \mathrm{e}-02$

$9.345821 \mathrm{e}-02$

$1.099387 \mathrm{e}-01$

$1.179615 \mathrm{e}-01$

$1.013862 \mathrm{e}-01$

$3.080654 \mathrm{e}-02$

$1.102220 \mathrm{e}-01$

$6.281384 \mathrm{e}-02$

$3.140442 \mathrm{e}-02$

$7.832849 \mathrm{e}-02$

$1.667767 \mathrm{e}-01$

$1.251194 \mathrm{e}-01$

$1.930055 \mathrm{e}-01$

$1.873550 \mathrm{e}-01$

$1.069828 \mathrm{e}-01$

$1.133314 \mathrm{e}-01$

$1.816873 \mathrm{e}-12$

$3.668570 \mathrm{e}-02$

$1.704366 \mathrm{e}-01$

$1.657539 \mathrm{e}-01$

$8.372611 \mathrm{e}-02$

$1.025534 \mathrm{e}-01$

2.388265e-01

$3.731614 \mathrm{e}-02$

$5.242834 \mathrm{e}-03$

$2.958901 \mathrm{e}-04$

$1.180200 \mathrm{e}-04$

$1.511250 \mathrm{e}-06$

$1.507218 \mathrm{e}-06$

$2.054855 \mathrm{e}-04$

$2.118619 \mathrm{e}-04$

$1.027610 \mathrm{e}-01$
$1.120740 \mathrm{e}-03$

$4.746942 \mathrm{e}-01$

$6.023889 \mathrm{e}-01$

$2.645585 \mathrm{e}-01$

$1.718494 \mathrm{e}-03$

$3.996665 \mathrm{e}-01$

$1.773469 \mathrm{e}-04$

$7.845697 \mathrm{e}-01$

$6.648547 \mathrm{e}-01$

$5.541719 \mathrm{e}-01$

$2.020316 \mathrm{e}-01$

$1.223809 \mathrm{e}-01$

$7.139773 \mathrm{e}-01$

$2.120017 \mathrm{e}-01$

$2.453794 \mathrm{e}-01$

$1.687936 \mathrm{e}-01$

5.474691e-01

$4.951425 \mathrm{e}-02$

$4.335959 \mathrm{e}-04$

$1.706763 \mathrm{e}-01$

$1.646449 \mathrm{e}-03$

5.377771e-02

6.495817e-01

9.471643e-01

$7.571110 \mathrm{e}-01$

$3.662980 \mathrm{e}-02$

$6.702879 \mathrm{e}-01$

$2.088108 \mathrm{e}-01$

9.190013e-01

7.975343e-02

$2.535380 \mathrm{e}-01$

$2.050760 \mathrm{e}-01$

$6.311767 \mathrm{e}-01$

4.849914e-01

$1.146947 \mathrm{e}-01$

$5.981321 \mathrm{e}-01$

$1.361746 \mathrm{e}-02$

8.905122e-01

$3.643571 \mathrm{e}-02$

6.315770e-01

$3.511898 \mathrm{e}-01$

$3.991927 \mathrm{e}-04$

$4.467505 \mathrm{e}-05$

$4.798314 \mathrm{e}-01$

$5.424200 \mathrm{e}-03$

$1.506744 \mathrm{e}-01$

$5.223145 \mathrm{e}-07$

$6.465224 \mathrm{e}-01$

$1.094790 \mathrm{e}-03$

$4.043268 \mathrm{e}-02$

$9.701704 \mathrm{e}-01$

$1.798616 \mathrm{e}-01$

3.250046e-01

$8.120352 \mathrm{e}-01$

7.801452e-02

$8.334260 \mathrm{e}-01$

$2.642669 \mathrm{e}-01$

$4.711246 \mathrm{e}-01$

$4.079520 \mathrm{e}-01$

$8.581338 \mathrm{e}-01$

$5.503726 \mathrm{e}-02$

$6.369445 \mathrm{e}-07$

$4.335089 \mathrm{e}-03$

$1.226567 \mathrm{e}-01$

$1.746434 \mathrm{e}-03$

$1.971511 \mathrm{e}-02$

6.094490e-02

$3.888245 \mathrm{e}-01$

$1.166201 \mathrm{e}-06$

$3.425393 \mathrm{e}-02$
**

*** 
969 Uncertain_Probability Condensed_matter_physics

970 Uncertain_Probability Biomedical_engineering

971 Uncertain_Probability Hydrology

972 Uncertain_Probability Atomic_physics

973 Uncertain_Probability Development_economics

974 Uncertain_Probability Optics

975 Uncertain_Probability Public_policy

976 Uncertain_Probability Lung_cancer

977 Uncertain_Probability Electronic_engineering

978 Uncertain_Probability Artificial_intelligence

979 Uncertain_Probability Machine_learning

980 Uncertain_Probability Gender_studies

981 Uncertain_Probability Chemical_engineering

982 Uncertain_Probability Biophysics

983 Uncertain_Probability Analytical_chemistry

984 Uncertain_Probability Computer_science

985 Uncertain_Probability Radiology

986 Uncertain_Probability Urology

987 Uncertain_Probability Architecture

988 Uncertain_Probability Quantum_mechanics

989 Uncertain_Probability Particle_physics

990 Uncertain_Probability Materials_science

991 Uncertain_Probability Computational_biology

992 Uncertain_Probability Agronomy

993 Uncertain_Probability Environmental_resource management

994 Uncertain_Probability Molecular_biology

995 Uncertain_Probability Dermatology

996 Uncertain_Probability Engineering

997 Uncertain_Probability Surgery

998 Uncertain_Probability Public health

999 Uncertain_Probability Molecular_physics

1000 Uncertain_Probability Bioinformatics

1001 Uncertain_Probability Physics

1002 Uncertain_Probability Cognitive_psychology

1003 Uncertain_Probability Oncology

1004 Uncertain_Probability Pathology

1005 Uncertain_Probability Biochemistry

1006 Uncertain_Probability Social_science

1007 Uncertain_Probability Climatology

1008 Uncertain_Probability Pharmacology

1009 Uncertain_Probability Agriculture

1010 Uncertain_Probability Neuroscience

1011 Uncertain_Probability Fishery

1012 Uncertain_Probability Virology

1013 Uncertain_Probability Microbiology

1014 Uncertain_Probability Communication

1015 Uncertain_Probability Endocrinology

1016 Uncertain_Probability Epigenetics

1017 Uncertain_Probability Marketing

1018 Uncertain_Probability Global_health

1019 Uncertain_Probability Agroforestry

1020 Uncertain_Probability Chemistry

1021 Uncertain_Probability Mathematics

1022 Uncertain_Probability Gynecology

1023 Uncertain_Probability Gerontology

1024 Uncertain_Probability Meteorology

1025 Uncertain_Probability Veterinary_medicine

1026 Uncertain_Probability Government

1027 Uncertain_Probability Dentistry

1028 Uncertain_Probability Microeconomics

1029 Uncertain_Probability Sociology

1030 Uncertain_Probability Emergency_medicine

1031 Uncertain_Probability Pediatrics

1032 Uncertain_Probability Advertising

1033 Uncertain_Probability Oceanography

1034 Uncertain_Probability Developmental_psychology

1035 Uncertain_Probability Internal_medicine

1036 Uncertain_Probability Cardiology

1037 Uncertain_Probability Environmental_engineering
$-2.198914 \mathrm{e}-01$

$-1.356467 \mathrm{e}-01$

$-1.574607 \mathrm{e}-01$

$-2.279647 \mathrm{e}-01$

$-2.707660 \mathrm{e}-01$

$4.712471 \mathrm{e}-01$

$-2.746660 \mathrm{e}-01$

$8.628951 \mathrm{e}-02$

$-2.269317 \mathrm{e}-01$

$-8.404610 \mathrm{e}-02$

$1.421098 \mathrm{e}+00$

$-2.983400 \mathrm{e}-01$

$-2.293171 \mathrm{e}-01$

$1.753442 \mathrm{e}-02$

$1.084363 \mathrm{e}-01$

$-1.022481 \mathrm{e}-01$

$5.072120 \mathrm{e}-02$

$-1.738161 \mathrm{e}-01$

$-3.161286 \mathrm{e}-01$

$-1.564942 \mathrm{e}+00$

$1.404331 \mathrm{e}+00$

$-1.058571 \mathrm{e}-01$

$3.006389 \mathrm{e}-02$

$7.525581 \mathrm{e}-01$

$-3.349536 \mathrm{e}-04$

$-1.695191 \mathrm{e}-01$

$-1.454835 \mathrm{e}-01$

$1.050522 \mathrm{e}-01$

$-5.300246 \mathrm{e}-02$

$7.830558 \mathrm{e}-02$

$7.751471 \mathrm{e}-01$

$2.884182 \mathrm{e}-02$

$-1.955471 \mathrm{e}-01$

$6.335820 \mathrm{e}-03$

$-4.123643 \mathrm{e}-02$

$-7.967555 \mathrm{e}-03$

$1.289871 \mathrm{e}-01$

$-2.549511 \mathrm{e}-02$

$1.274779 \mathrm{e}-01$

$2.743227 \mathrm{e}-01$

$1.816577 \mathrm{e}-01$

$-2.011369 \mathrm{e}-01$

$-9.168263 \mathrm{e}-02$

$-9.027212 \mathrm{e}-02$

$-1.559873 \mathrm{e}-02$

$5.693697 \mathrm{e}-02$

6.499492e-02

$4.674699 \mathrm{e}-02$

$-1.511546 \mathrm{e}-02$

$3.787636 \mathrm{e}-01$

$-2.422385 \mathrm{e}-01$

$-2.383692 \mathrm{e}-02$

$-6.605296 \mathrm{e}-01$

6.249617e-02

$-5.766104 \mathrm{e}-02$

-2.162686e-02

$7.791393 \mathrm{e}-01$

$-1.322401 \mathrm{e}-01$

$4.520162 \mathrm{e}-01$

$-1.542488 \mathrm{e}-01$

$-2.859694 \mathrm{e}-01$

$-8.611641 \mathrm{e}-02$

$-6.094757 \mathrm{e}-02$

$7.636235 \mathrm{e}-02$

$1.628944 \mathrm{e}-01$

$4.093758 \mathrm{e}-02$

$-3.554110 \mathrm{e}-02$

2.605576e-02

$-6.175185 \mathrm{e}-02$
$2.093669 \mathrm{e}-01$

$1.978016 \mathrm{e}-01$

$1.734878 \mathrm{e}-01$

$2.462479 \mathrm{e}-01$

$2.802235 \mathrm{e}-01$

$3.057178 \mathrm{e}-01$

$1.880922 \mathrm{e}-01$

4.465271e-02

$5.137966 \mathrm{e}-01$

$2.598096 \mathrm{e}-01$

$2.752173 \mathrm{e}-01$

$1.654655 \mathrm{e}-01$

$1.931587 \mathrm{e}-01$

$1.185080 \mathrm{e}-01$

2.101446e-01

$1.384125 \mathrm{e}-01$

$4.932645 \mathrm{e}-02$

$7.457768 \mathrm{e}-02$

$1.693253 \mathrm{e}-01$

$6.706601 \mathrm{e}-01$

$5.803593 \mathrm{e}-01$

$1.000304 \mathrm{e}-01$

$2.462681 \mathrm{e}-01$

$1.318497 \mathrm{e}-01$

$7.605521 \mathrm{e}-02$

$4.926034 \mathrm{e}-02$

$6.710043 \mathrm{e}-02$

$1.786432 \mathrm{e}-01$

$2.505216 \mathrm{e}-02$

$3.414137 \mathrm{e}-02$

$2.464528 \mathrm{e}-01$

$1.129317 \mathrm{e}-01$

$1.282568 \mathrm{e}-01$

$6.430769 \mathrm{e}-02$

$4.424295 \mathrm{e}-02$

$4.588720 \mathrm{e}-02$

$6.283979 \mathrm{e}-02$

$2.501209 \mathrm{e}-01$

$6.947653 \mathrm{e}-02$

$1.002892 \mathrm{e}-01$

8.105492e-02

$5.393076 \mathrm{e}-02$

$1.153944 \mathrm{e}-01$

5.203463e-02

$7.739787 \mathrm{e}-02$

$7.947642 \mathrm{e}-02$

$2.562771 \mathrm{e}-02$

$7.675768 \mathrm{e}-02$

$8.280261 \mathrm{e}-02$

$1.216342 \mathrm{e}-01$

$1.062758 \mathrm{e}-01$

$8.877427 \mathrm{e}-02$

$2.587135 \mathrm{e}-01$

$4.244360 \mathrm{e}-02$

$4.498158 \mathrm{e}-02$

$1.344838 \mathrm{e}-01$

$3.070121 \mathrm{e}-01$

$9.029177 \mathrm{e}-02$

$1.664582 \mathrm{e}-01$

$1.414857 \mathrm{e}-01$

$1.765509 \mathrm{e}-01$

$3.764246 \mathrm{e}-02$

$3.023622 \mathrm{e}-02$

8.194060e-02

$1.248852 \mathrm{e}-01$

$4.514958 \mathrm{e}-02$

$2.470047 \mathrm{e}-02$

3.930417e-02

$1.185218 \mathrm{e}-01$ 2.936143e-01 $4.928694 \mathrm{e}-01$

3.640967e-01

$3.545915 \mathrm{e}-01$

3.339370e-01

$1.232329 \mathrm{e}-01$

$1.442390 \mathrm{e}-01$

$5.332455 \mathrm{e}-02$

$6.587309 \mathrm{e}-01$

$7.463285 \mathrm{e}-01$

2.458761e-07

$7.140586 \mathrm{e}-02$

$2.351723 \mathrm{e}-01$

$8.823768 \mathrm{e}-01$

$6.058578 \mathrm{e}-01$

$4.600900 \mathrm{e}-01$

$3.038392 \mathrm{e}-01$

$1.978584 \mathrm{e}-02$

$6.192546 \mathrm{e}-02$

$1.964043 \mathrm{e}-02$

$1.554429 \mathrm{e}-02$

$2.899614 \mathrm{e}-01$

$9.028392 \mathrm{e}-01$

$1.170073 \mathrm{e}-08$

9.964861e-01

$5.807595 \mathrm{e}-04$

$3.016557 \mathrm{e}-02$

$5.565050 \mathrm{e}-01$

$3.439057 \mathrm{e}-02$

$2.183108 \mathrm{e}-02$

$1.663373 \mathrm{e}-03$

7.984246e-01

$1.273699 \mathrm{e}-01$

$9.215181 \mathrm{e}-01$

$3.513304 \mathrm{e}-01$

$8.621562 \mathrm{e}-01$

$4.012798 \mathrm{e}-02$

$9.188129 \mathrm{e}-01$

6.655302e-02

$6.240440 \mathrm{e}-03$

$2.503190 \mathrm{e}-02$

$1.926414 \mathrm{e}-04$

4.269098e-01

$8.279154 \mathrm{e}-02$

$8.402798 \mathrm{e}-01$

$4.737567 \mathrm{e}-01$

$1.122043 \mathrm{e}-02$

$5.425216 \mathrm{e}-01$

$8.551555 \mathrm{e}-01$

$1.849953 \mathrm{e}-03$

$2.266304 \mathrm{e}-02$

$7.883098 \mathrm{e}-01$

$1.068721 \mathrm{e}-02$

$1.409229 \mathrm{e}-01$

$1.999072 \mathrm{e}-01$

$8.722424 \mathrm{e}-01$

$1.116630 \mathrm{e}-02$

$1.430578 \mathrm{e}-01$

$6.626408 \mathrm{e}-03$

$2.756417 \mathrm{e}-01$

$1.053089 \mathrm{e}-01$

$2.216820 \mathrm{e}-02$

$4.385034 \mathrm{e}-02$

$3.513934 \mathrm{e}-01$

$1.921361 \mathrm{e}-01$

$3.645768 \mathrm{e}-01$

$1.502074 \mathrm{e}-01$

$5.073896 \mathrm{e}-01$

$6.023641 \mathrm{e}-01$

*** 
1038 Uncertain_Probability General_surgery

1039 Uncertain_Probability Environmental_science

1040 Uncertain_Probability Geophysics

1041 Uncertain_Probability Immunology

1042 Uncertain_Probability Clinical_psychology

1043 Uncertain_Probability Toxicology

1044 Uncertain_Probability Ecology

1045 Uncertain_Probability Biology

1046 Uncertain_Probability Epidemiology

1047 Uncertain_Probability Physical_therapy

1048 Uncertain_Probability Physiology

1049 Uncertain_Probability Statistics

1050 Uncertain_Probability Psychopathology

1051 Uncertain_Probability Animal_science

1052 Uncertain_Probability Nursing

1053 Uncertain_Probability Seismology

1054 Uncertain_Probability History

1055 Uncertain_Probability Psychiatry

1056 Uncertain_Probability Zoology

1057 Uncertain_Probability Psychology

1058 Uncertain_Probability Etiology

1059 Uncertain_Probability Geomorphology

1060 Uncertain_Probability Atmospheric_sciences

1061 Uncertain_Probability Geography

1062 Uncertain_Probability Demography

1063 Uncertain_Probability Geology

1064 Uncertain_Probability Medicine

1065 Uncertain_Probability Family_medicine

1066 Uncertain_Probability Evolutionary_biology

1067 Uncertain_Probability Astrobiology

1068 Uncertain_Probability Astronomy

1069 Uncertain_Probability Astrophysics

1070 Uncertain_Probability Geochemistry

1071 Uncertain_Probability Business

1072 Uncertain_Probability Finance

1073 Uncertain_Probability Management_science

1074 Uncertain_Probability Social_psychology

1075 Uncertain_Probability Political_science

1076 Uncertain_Probability Archaeology

1077 Uncertain_Probability Economics

1078 Uncertain_Probability Paleontology

1079 Uncertain_Probability Criminology

1080 Uncertain_Condition Intercept

1081 Uncertain_Condition source[T.news]

1082 Uncertain_Condition finding_len

1083 Uncertain_Condition flesch_reading_ease

1084 Uncertain_Condition first_author_rank

1085 Uncertain_Condition affi_rank

1086 Uncertain_Condition num_authors

1087 Uncertain_Condition journal_impact

1088 Uncertain_Condition Law

1089 Uncertain_Condition Condensed_matter_physics

1090 Uncertain_Condition Biomedical_engineering

1091 Uncertain_Condition Hydrology

1092 Uncertain_Condition Atomic_physics

1093 Uncertain_Condition Development_economics

1094 Uncertain_Condition Optics

1095 Uncertain_Condition Public_policy

1096 Uncertain_Condition Lung_cancer

1097 Uncertain_Condition Electronic_engineering

1098 Uncertain_Condition Artificial_intelligence

1099 Uncertain_Condition Machine_learning

1100 Uncertain_Condition Gender_studies

1101 Uncertain_Condition Chemical_engineering

1102 Uncertain_Condition Biophysics

1103 Uncertain_Condition Analytical_chemistry

1104 Uncertain_Condition Computer_science

1105 Uncertain_Condition Radiology

1106 Uncertain_Condition Urology

1107 Uncertain_Condition Architecture
$-7.962692 \mathrm{e}-02$

$8.932392 \mathrm{e}-02$

$7.440402 \mathrm{e}-02$

$6.618625 \mathrm{e}-02$

$-9.215721 \mathrm{e}-02$

$-2.213692 \mathrm{e}-01$

$8.688322 \mathrm{e}-02$

$1.721073 \mathrm{e}-02$

$1.898233 \mathrm{e}-02$

$-2.833412 \mathrm{e}-02$

$-1.229246 \mathrm{e}-01$

$1.634806 \mathrm{e}-01$

$-9.333176 \mathrm{e}-02$

$5.686181 \mathrm{e}-02$

$1.016668 \mathrm{e}-02$

$4.151404 \mathrm{e}-01$

$-3.462328 \mathrm{e}-01$

$-3.184194 \mathrm{e}-02$

$2.374243 \mathrm{e}-02$

$-9.577284 \mathrm{e}-03$

$-1.616802 \mathrm{e}-01$

$3.686531 \mathrm{e}-01$

$7.436546 \mathrm{e}-02$

$-1.592509 \mathrm{e}-01$

5.428162e-02

$-4.776377 \mathrm{e}-02$

$-1.335884 \mathrm{e}-01$

$-1.483964 \mathrm{e}-02$

$-6.045480 \mathrm{e}-02$

$-3.944000 \mathrm{e}-02$

$2.974058 \mathrm{e}-01$

$1.921862 \mathrm{e}-02$

$4.483335 \mathrm{e}-01$

1.197080e-01

$-3.773369 \mathrm{e}-02$

$-8.347154 \mathrm{e}-13$

$-2.219061 \mathrm{e}-02$

$-2.748613 \mathrm{e}-01$

$-2.405449 \mathrm{e}-01$

$5.350383 \mathrm{e}-02$

$2.257395 \mathrm{e}-01$

$-3.320738 \mathrm{e}-01$

$0.000000 \mathrm{e}+00$

$0.000000 \mathrm{e}+00$

$0.000000 \mathrm{e}+00$

$0.000000 \mathrm{e}+00$

$0.000000 \mathrm{e}+00$

$0.000000 \mathrm{e}+00$

$0.000000 \mathrm{e}+00$

$0.000000 \mathrm{e}+00$

$0.000000 \mathrm{e}+00$

$0.000000 \mathrm{e}+00$

$0.000000 \mathrm{e}+00$

$0.000000 \mathrm{e}+00$

$0.000000 \mathrm{e}+00$

$0.000000 \mathrm{e}+00$

$0.000000 \mathrm{e}+00$

$0.000000 \mathrm{e}+00$

$0.000000 \mathrm{e}+00$

$0.000000 \mathrm{e}+00$

$0.000000 \mathrm{e}+00$

$0.000000 \mathrm{e}+00$

$0.000000 \mathrm{e}+00$

$0.000000 \mathrm{e}+00$

$0.000000 \mathrm{e}+00$

$0.000000 \mathrm{e}+00$

$0.000000 \mathrm{e}+00$

$0.000000 \mathrm{e}+00$

$0.000000 \mathrm{e}+00$

$0.000000 \mathrm{e}+00$ 4.583841e-02

9.336765e-02

$2.424030 \mathrm{e}-01$

$3.241235 \mathrm{e}-02$

4.678780e-02

$1.510432 \mathrm{e}-01$

$3.604414 \mathrm{e}-02$

$6.910098 \mathrm{e}-02$

$3.794267 \mathrm{e}-02$

$2.468372 \mathrm{e}-02$

$9.530415 \mathrm{e}-02$

$3.050575 \mathrm{e}-01$

$1.106242 \mathrm{e}-01$

$1.728219 \mathrm{e}-01$

$4.483078 \mathrm{e}-02$

$1.895768 \mathrm{e}-01$

5.304454e-01

$3.142701 \mathrm{e}-02$

$8.242409 \mathrm{e}-02$

$6.857302 \mathrm{e}-02$

$8.791349 \mathrm{e}-02$

$1.034162 \mathrm{e}-01$

$1.109630 \mathrm{e}-01$

9.537114e-02

$2.897884 \mathrm{e}-02$

$1.036828 \mathrm{e}-01$

$5.908720 \mathrm{e}-02$

$2.954125 \mathrm{e}-02$

$7.368139 \mathrm{e}-02$

$1.568821 \mathrm{e}-01$

$1.176963 \mathrm{e}-01$

$1.815548 \mathrm{e}-01$

$1.762395 \mathrm{e}-01$

$1.006357 \mathrm{e}-01$

$1.066076 \mathrm{e}-01$

$1.709081 \mathrm{e}-12$

$3.450919 \mathrm{e}-02$

$1.603249 \mathrm{e}-01$

$1.559200 \mathrm{e}-01$

$7.875878 \mathrm{e}-02$

9.646907e-02

$2.246573 \mathrm{e}-01$

$0.000000 \mathrm{e}+00$

$0.000000 \mathrm{e}+00$

$0.000000 \mathrm{e}+00$

$0.000000 \mathrm{e}+00$

$0.000000 \mathrm{e}+00$

$0.000000 \mathrm{e}+00$

$0.000000 \mathrm{e}+00$

$0.000000 \mathrm{e}+00$

$0.000000 \mathrm{e}+00$

$0.000000 \mathrm{e}+00$

$0.000000 \mathrm{e}+00$

$0.000000 \mathrm{e}+00$

$0.000000 \mathrm{e}+00$

$0.000000 \mathrm{e}+00$

$0.000000 \mathrm{e}+00$

$0.000000 \mathrm{e}+00$

$0.000000 \mathrm{e}+00$

$0.000000 \mathrm{e}+00$

$0.000000 \mathrm{e}+00$

$0.000000 \mathrm{e}+00$

$0.000000 \mathrm{e}+00$

$0.000000 \mathrm{e}+00$

$0.000000 \mathrm{e}+00$

$0.000000 \mathrm{e}+00$

$0.000000 \mathrm{e}+00$

$0.000000 \mathrm{e}+00$

$0.000000 \mathrm{e}+00$

$0.000000 \mathrm{e}+00$ 8.238921e-02

3.387413e-01

$7.588913 \mathrm{e}-01$

$4.117085 \mathrm{e}-02$

$4.889565 \mathrm{e}-02$

$1.427810 \mathrm{e}-01$

$1.594582 \mathrm{e}-02$

8.033133e-01

$6.168794 \mathrm{e}-01$

$2.510362 \mathrm{e}-01$

$1.971382 \mathrm{e}-01$

$5.920361 \mathrm{e}-01$

$3.988622 \mathrm{e}-01$

7.421460e-01

$8.205991 \mathrm{e}-01$

2.855437e-02

$5.139478 \mathrm{e}-01$

$3.109820 \mathrm{e}-01$

$7.733115 \mathrm{e}-01$

$8.889265 \mathrm{e}-01$

$6.592572 \mathrm{e}-02$

$3.655020 \mathrm{e}-04$

$5.027534 \mathrm{e}-01$

$9.498255 \mathrm{e}-02$

$6.107044 \mathrm{e}-02$

$6.450414 \mathrm{e}-01$

$2.378376 \mathrm{e}-02$

$6.154395 \mathrm{e}-01$

$4.119522 \mathrm{e}-01$

8.015096e-01

$1.151939 \mathrm{e}-02$

9.156984e-01

$1.097407 \mathrm{e}-02$

$2.342574 \mathrm{e}-01$

$7.233826 \mathrm{e}-01$

$6.252747 \mathrm{e}-01$

$5.202129 \mathrm{e}-01$

8.647861e-02

$1.229166 \mathrm{e}-01$

$4.969358 \mathrm{e}-01$

$1.929774 \mathrm{e}-02$

$1.393958 \mathrm{e}-01$

$\mathrm{NaN}$

$\mathrm{NaN}$

$\mathrm{NaN}$

$\mathrm{NaN}$

$\mathrm{NaN}$

$\mathrm{NaN}$

$\mathrm{NaN}$

$\mathrm{NaN}$

$\mathrm{NaN}$

$\mathrm{NaN}$

$\mathrm{NaN}$

$\mathrm{NaN}$

$\mathrm{NaN}$

$\mathrm{NaN}$

$\mathrm{NaN}$

$\mathrm{NaN}$

$\mathrm{NaN}$

$\mathrm{NaN}$

$\mathrm{NaN}$

$\mathrm{NaN}$

$\mathrm{NaN}$

$\mathrm{NaN}$

$\mathrm{NaN}$

$\mathrm{NaN}$

$\mathrm{NaN}$

$\mathrm{NaN}$

$\mathrm{NaN}$

$\mathrm{NaN}$

\section{*}

*** 
1108 Uncertain_Condition Quantum_mechanics

1109 Uncertain_Condition Particle_physics

1110 Uncertain_Condition Materials_science

1111 Uncertain_Condition Computational_biology

1112 Uncertain_Condition Agronomy

1113 Uncertain_Condition Environmental_resource management

1114 Uncertain_Condition Molecular_biology

1115 Uncertain_Condition Dermatology

1116 Uncertain_Condition Engineering

1117 Uncertain_Condition Surgery

1118 Uncertain_Condition Public_health

1119 Uncertain_Condition Molecular_physics

1120 Uncertain_Condition Bioinformatics

1121 Uncertain_Condition Physics

1122 Uncertain_Condition Cognitive_psychology

1123 Uncertain_Condition Oncology

1124 Uncertain_Condition Pathology

1125 Uncertain_Condition Biochemistry

1126 Uncertain_Condition Social_science

1127 Uncertain_Condition Climatology

1128 Uncertain_Condition Pharmacology

1129 Uncertain_Condition Agriculture

1130 Uncertain_Condition Neuroscience

1131 Uncertain_Condition Fishery

1132 Uncertain_Condition Virology

1133 Uncertain_Condition Microbiology

1134 Uncertain_Condition Communication

1135 Uncertain_Condition Endocrinology

1136 Uncertain_Condition Epigenetics

1137 Uncertain_Condition Marketing

1138 Uncertain_Condition Global_health

1139 Uncertain_Condition Agroforestry

1140 Uncertain_Condition Chemistry

1141 Uncertain_Condition Mathematics

1142 Uncertain_Condition Gynecology

1143 Uncertain_Condition Gerontology

1144 Uncertain_Condition Meteorology

1145 Uncertain_Condition Veterinary_medicine

1146 Uncertain_Condition Government

1147 Uncertain_Condition Dentistry

1148 Uncertain_Condition Microeconomics

1149 Uncertain_Condition Sociology

1150 Uncertain_Condition Emergency_medicine

1151 Uncertain_Condition Pediatrics

1152 Uncertain_Condition Advertising

1153 Uncertain_Condition Oceanography

1154 Uncertain_Condition Developmental_psychology

1155 Uncertain_Condition Internal_medicine

1156 Uncertain_Condition Cardiology

1157 Uncertain_Condition Environmental_engineering

1158 Uncertain_Condition General_surgery

1159 Uncertain_Condition Environmental_science

1160 Uncertain_Condition Geophysics

1161 Uncertain_Condition Immunology

1162 Uncertain_Condition Clinical_psychology

1163 Uncertain_Condition Toxicology

1164 Uncertain_Condition Ecology

1165 Uncertain_Condition Biology

1166 Uncertain_Condition Epidemiology

1167 Uncertain_Condition Physical_therapy

1168 Uncertain_Condition Physiology

1169 Uncertain_Condition Statistics

1170 Uncertain_Condition Psychopathology

1171 Uncertain_Condition Animal_science

1172 Uncertain_Condition Nursing

1173 Uncertain_Condition Seismology

1174 Uncertain_Condition History

1175 Uncertain_Condition Psychiatry

1176 Uncertain_Condition Zoology
$0.000000 \mathrm{e}+00$

$0.000000 \mathrm{e}+00$

$0.000000 \mathrm{e}+00$

$0.000000 \mathrm{e}+00$

$0.000000 \mathrm{e}+00$

$0.000000 \mathrm{e}+00$

$0.000000 \mathrm{e}+00$

$0.000000 \mathrm{e}+00$

$0.000000 \mathrm{e}+00$

$0.000000 \mathrm{e}+00$

$0.000000 \mathrm{e}+00$

$0.000000 \mathrm{e}+00$

$0.000000 \mathrm{e}+00$

$0.000000 \mathrm{e}+00$

$0.000000 \mathrm{e}+00$

$0.000000 \mathrm{e}+00$

$0.000000 \mathrm{e}+00$

$0.000000 \mathrm{e}+00$

$0.000000 \mathrm{e}+00$

$0.000000 \mathrm{e}+00$

$0.000000 \mathrm{e}+00$

$0.000000 \mathrm{e}+00$

$0.000000 \mathrm{e}+00$

$0.000000 \mathrm{e}+00$

$0.000000 \mathrm{e}+00$

$0.000000 \mathrm{e}+00$

$0.000000 \mathrm{e}+00$

$0.000000 \mathrm{e}+00$

$0.000000 \mathrm{e}+00$

$0.000000 \mathrm{e}+00$

$0.000000 \mathrm{e}+00$

$0.000000 \mathrm{e}+00$

$0.000000 \mathrm{e}+00$

$0.000000 \mathrm{e}+00$

$0.000000 \mathrm{e}+00$

$0.000000 \mathrm{e}+00$

$0.000000 \mathrm{e}+00$

$0.000000 \mathrm{e}+00$

$0.000000 \mathrm{e}+00$

$0.000000 \mathrm{e}+00$

$0.000000 \mathrm{e}+00$

$0.000000 \mathrm{e}+00$

$0.000000 \mathrm{e}+00$

$0.000000 \mathrm{e}+00$

$0.000000 \mathrm{e}+00$

$0.000000 \mathrm{e}+00$

$0.000000 \mathrm{e}+00$

$0.000000 \mathrm{e}+00$

$0.000000 \mathrm{e}+00$

$0.000000 \mathrm{e}+00$

$0.000000 \mathrm{e}+00$

$0.000000 \mathrm{e}+00$

$0.000000 \mathrm{e}+00$

$0.000000 \mathrm{e}+00$

$0.000000 \mathrm{e}+00$

$0.000000 \mathrm{e}+00$

$0.000000 \mathrm{e}+00$

$0.000000 \mathrm{e}+00$

$0.000000 \mathrm{e}+00$

$0.000000 \mathrm{e}+00$

$0.000000 \mathrm{e}+00$

$0.000000 \mathrm{e}+00$

$0.000000 \mathrm{e}+00$

$0.000000 \mathrm{e}+00$

$0.000000 \mathrm{e}+00$

$0.000000 \mathrm{e}+00$

$0.000000 \mathrm{e}+00$

$0.000000 \mathrm{e}+00$

$0.000000 \mathrm{e}+00$
$0.000000 \mathrm{e}+00$

$0.000000 \mathrm{e}+00$

$0.000000 \mathrm{e}+00$

$0.000000 \mathrm{e}+00$

$0.000000 \mathrm{e}+00$

$0.000000 \mathrm{e}+00$

$0.000000 \mathrm{e}+00$

$0.000000 \mathrm{e}+00$

$0.000000 \mathrm{e}+00$

$0.000000 \mathrm{e}+00$

$0.000000 \mathrm{e}+00$

$0.000000 \mathrm{e}+00$

$0.000000 \mathrm{e}+00$

$0.000000 \mathrm{e}+00$

$0.000000 \mathrm{e}+00$

$0.000000 \mathrm{e}+00$

$0.000000 \mathrm{e}+00$

$0.000000 \mathrm{e}+00$

$0.000000 \mathrm{e}+00$

$0.000000 \mathrm{e}+00$

$0.000000 \mathrm{e}+00$

$0.000000 \mathrm{e}+00$

$0.000000 \mathrm{e}+00$

$0.000000 \mathrm{e}+00$

$0.000000 \mathrm{e}+00$

$0.000000 \mathrm{e}+00$

$0.000000 \mathrm{e}+00$

$0.000000 \mathrm{e}+00$

$0.000000 \mathrm{e}+00$

$0.000000 \mathrm{e}+00$

$0.000000 \mathrm{e}+00$

$0.000000 \mathrm{e}+00$

$0.000000 \mathrm{e}+00$

$0.000000 \mathrm{e}+00$

$0.000000 \mathrm{e}+00$

$0.000000 \mathrm{e}+00$

$0.000000 \mathrm{e}+00$

$0.000000 \mathrm{e}+00$

$0.000000 \mathrm{e}+00$

$0.000000 \mathrm{e}+00$

$0.000000 \mathrm{e}+00$

$0.000000 \mathrm{e}+00$

$0.000000 \mathrm{e}+00$

$0.000000 \mathrm{e}+00$

$0.000000 \mathrm{e}+00$

$0.000000 \mathrm{e}+00$

$0.000000 \mathrm{e}+00$

$0.000000 \mathrm{e}+00$

$0.000000 \mathrm{e}+00$

$0.000000 \mathrm{e}+00$

$0.000000 \mathrm{e}+00$

$0.000000 \mathrm{e}+00$

$0.000000 \mathrm{e}+00$

$0.000000 \mathrm{e}+00$

$0.000000 \mathrm{e}+00$

$0.000000 \mathrm{e}+00$

$0.000000 \mathrm{e}+00$

$0.000000 \mathrm{e}+00$

$0.000000 \mathrm{e}+00$

$0.000000 \mathrm{e}+00$

$0.000000 \mathrm{e}+00$

$0.000000 \mathrm{e}+00$

$0.000000 \mathrm{e}+00$

$0.000000 \mathrm{e}+00$

$0.000000 \mathrm{e}+00$

$0.000000 \mathrm{e}+00$

$0.000000 \mathrm{e}+00$

$0.000000 \mathrm{e}+00$

$0.000000 \mathrm{e}+00$
$\mathrm{NaN}$

$\mathrm{NaN}$

$\mathrm{NaN}$

$\mathrm{NaN}$

$\mathrm{NaN}$

$\mathrm{NaN}$

$\mathrm{NaN}$

$\mathrm{NaN}$

$\mathrm{NaN}$

$\mathrm{NaN}$

$\mathrm{NaN}$

$\mathrm{NaN}$

$\mathrm{NaN}$

$\mathrm{NaN}$

$\mathrm{NaN}$

$\mathrm{NaN}$

$\mathrm{NaN}$

$\mathrm{NaN}$

$\mathrm{NaN}$

$\mathrm{NaN}$

$\mathrm{NaN}$

$\mathrm{NaN}$

$\mathrm{NaN}$

$\mathrm{NaN}$

$\mathrm{NaN}$

$\mathrm{NaN}$

$\mathrm{NaN}$

$\mathrm{NaN}$

$\mathrm{NaN}$

$\mathrm{NaN}$

$\mathrm{NaN}$

$\mathrm{NaN}$

$\mathrm{NaN}$

$\mathrm{NaN}$

$\mathrm{NaN}$

$\mathrm{NaN}$

$\mathrm{NaN}$

$\mathrm{NaN}$

$\mathrm{NaN}$

$\mathrm{NaN}$

$\mathrm{NaN}$

$\mathrm{NaN}$

$\mathrm{NaN}$

$\mathrm{NaN}$

$\mathrm{NaN}$

$\mathrm{NaN}$

$\mathrm{NaN}$

$\mathrm{NaN}$

$\mathrm{NaN}$

$\mathrm{NaN}$

$\mathrm{NaN}$

$\mathrm{NaN}$

$\mathrm{NaN}$

$\mathrm{NaN}$

$\mathrm{NaN}$

$\mathrm{NaN}$

$\mathrm{NaN}$

$\mathrm{NaN}$

$\mathrm{NaN}$

$\mathrm{NaN}$

$\mathrm{NaN}$

$\mathrm{NaN}$

$\mathrm{NaN}$

$\mathrm{NaN}$

$\mathrm{NaN}$

$\mathrm{NaN}$

$\mathrm{NaN}$

$\mathrm{NaN}$

$\mathrm{NaN}$ 
1177 Uncertain_Condition Psychology

1178 Uncertain_Condition Etiology

1179 Uncertain_Condition Geomorphology

1180 Uncertain_Condition Atmospheric_sciences

1181 Uncertain_Condition Geography

1182 Uncertain_Condition Demography

1183 Uncertain_Condition Geology

1184 Uncertain_Condition Medicine

1185 Uncertain_Condition Family_medicine

1186 Uncertain_Condition Evolutionary_biology

1187 Uncertain_Condition Astrobiology

1188 Uncertain_Condition Astronomy

1189 Uncertain_Condition Astrophysics

1190 Uncertain_Condition Geochemistry

1191 Uncertain_Condition Business

1192 Uncertain_Condition Finance

1193 Uncertain_Condition Management_science

1194 Uncertain_Condition Social_psychology

1195 Uncertain_Condition Political_science

1196 Uncertain_Condition Archaeology

1197 Uncertain_Condition Economics

1198 Uncertain_Condition Paleontology

1199 Uncertain_Condition Criminology

1200 Uncertain_Suggestion Intercept

1201 Uncertain_Suggestion source[T.news]

1202 Uncertain_Suggestion finding_len

1203 Uncertain_Suggestion flesch_reading_ease

1204 Uncertain_Suggestion first_author_rank

1205 Uncertain_Suggestion affi_rank

1206 Uncertain_Suggestion num_authors

1207 Uncertain_Suggestion journal_impact

1208 Uncertain_Suggestion Law

1209 Uncertain_Suggestion Condensed_matter_physics

1210 Uncertain_Suggestion Biomedical_engineering

1211 Uncertain_Suggestion Hydrology

1212 Uncertain_Suggestion Atomic_physics

1213 Uncertain_Suggestion Development_economics

1214 Uncertain_Suggestion Optics

1215 Uncertain_Suggestion Public_policy

1216 Uncertain_Suggestion Lung_cancer

1217 Uncertain_Suggestion Electronic_engineering

1218 Uncertain_Suggestion Artificial_intelligence

1219 Uncertain_Suggestion Machine_learning

1220 Uncertain_Suggestion Gender_studies

1221 Uncertain_Suggestion Chemical_engineering

1222 Uncertain_Suggestion Biophysics

1223 Uncertain_Suggestion Analytical_chemistry

1224 Uncertain_Suggestion Computer_science

1225 Uncertain_Suggestion Radiology

1226 Uncertain_Suggestion Urology

1227 Uncertain_Suggestion Architecture

1228 Uncertain_Suggestion Quantum_mechanics

1229 Uncertain_Suggestion Particle_physics

1230 Uncertain_Suggestion Materials_science

1231 Uncertain_Suggestion Computational_biology

1232 Uncertain_Suggestion Agronomy

1233 Uncertain_Suggestion Environmental_resource management

1234 Uncertain_Suggestion Molecular_biology

1235 Uncertain_Suggestion Dermatology

1236 Uncertain_Suggestion Engineering

1237 Uncertain_Suggestion Surgery

1238 Uncertain_Suggestion Public_health

1239 Uncertain_Suggestion Molecular_physics

1240 Uncertain_Suggestion Bioinformatics

1241 Uncertain_Suggestion Physics

1242 Uncertain_Suggestion Cognitive_psychology

1243 Uncertain_Suggestion Oncology

1244 Uncertain_Suggestion Pathology

1245 Uncertain_Suggestion Biochemistry
$0.000000 \mathrm{e}+00$

$0.000000 \mathrm{e}+00$

$0.000000 \mathrm{e}+00$

$0.000000 \mathrm{e}+00$

$0.000000 \mathrm{e}+00$

$0.000000 \mathrm{e}+00$

$0.000000 \mathrm{e}+00$

$0.000000 \mathrm{e}+00$

$0.000000 \mathrm{e}+00$

$0.000000 \mathrm{e}+00$

$0.000000 \mathrm{e}+00$

$0.000000 \mathrm{e}+00$

$0.000000 \mathrm{e}+00$

$0.000000 \mathrm{e}+00$

$0.000000 \mathrm{e}+00$

$0.000000 \mathrm{e}+00$

$0.000000 \mathrm{e}+00$

$0.000000 \mathrm{e}+00$

$0.000000 \mathrm{e}+00$

$0.000000 \mathrm{e}+00$

$0.000000 \mathrm{e}+00$

$0.000000 \mathrm{e}+00$

$0.000000 \mathrm{e}+00$

$2.670386 \mathrm{e}-02$

$2.067624 \mathrm{e}-03$

$-2.445807 \mathrm{e}-04$

$-7.451400 \mathrm{e}-05$

$-6.141558 \mathrm{e}-07$

$-7.612485 \mathrm{e}-07$

$-4.797802 \mathrm{e}-05$

$-6.819403 \mathrm{e}-05$

$-1.560751 \mathrm{e}-02$

$-1.280859 \mathrm{e}-02$

$-3.277797 \mathrm{e}-02$

$-3.560857 \mathrm{e}-03$

$-5.982489 \mathrm{e}-03$

$-1.272990 \mathrm{e}-02$

$-2.384740 \mathrm{e}-02$

$-2.387632 \mathrm{e}-02$

$-1.331188 \mathrm{e}-02$

$-5.748026 \mathrm{e}-02$

$-7.498732 \mathrm{e}-03$

$-1.358001 \mathrm{e}-02$

$-6.031476 \mathrm{e}-03$

$-2.975552 \mathrm{e}-02$

$-2.138889 \mathrm{e}-02$

$-4.135868 \mathrm{e}-02$

$2.391564 \mathrm{e}-03$

$3.566238 \mathrm{e}-02$

$-8.399161 \mathrm{e}-03$

$-1.131125 \mathrm{e}-02$

$-2.345793 e-02$

$1.044366 \mathrm{e}-02$

$5.366930 \mathrm{e}-02$

$-1.665146 \mathrm{e}-02$

$3.898630 \mathrm{e}-02$

$-1.301216 \mathrm{e}-02$

$-1.223814 \mathrm{e}-02$

$-4.565215 \mathrm{e}-03$

$-4.783412 \mathrm{e}-03$

$9.958769 \mathrm{e}-03$

$1.179314 \mathrm{e}-02$

$-5.269004 \mathrm{e}-02$

$1.007092 \mathrm{e}-01$

$1.197093 \mathrm{e}-03$

$-2.037807 \mathrm{e}-02$

$2.811483 \mathrm{e}-03$

$1.741977 \mathrm{e}-02$

$-2.880700 \mathrm{e}-02$
$0.000000 \mathrm{e}+00$

$0.000000 \mathrm{e}+00$

$0.000000 \mathrm{e}+00$

$0.000000 \mathrm{e}+00$

$0.000000 \mathrm{e}+00$

$0.000000 \mathrm{e}+00$

$0.000000 \mathrm{e}+00$

$0.000000 \mathrm{e}+00$

$0.000000 \mathrm{e}+00$

$0.000000 \mathrm{e}+00$

$0.000000 \mathrm{e}+00$

$0.000000 \mathrm{e}+00$

$0.000000 \mathrm{e}+00$

$0.000000 \mathrm{e}+00$

$0.000000 \mathrm{e}+00$

$0.000000 \mathrm{e}+00$

$0.000000 \mathrm{e}+00$

$0.000000 \mathrm{e}+00$

$0.000000 \mathrm{e}+00$

$0.000000 \mathrm{e}+00$

$0.000000 \mathrm{e}+00$

$0.000000 \mathrm{e}+00$

$0.000000 \mathrm{e}+00$

$8.583111 \mathrm{e}-03$

$1.205908 \mathrm{e}-03$

$6.805788 \mathrm{e}-05$

$2.714587 \mathrm{e}-05$

$3.476037 \mathrm{e}-07$

3.466763e-07

$4.726386 \mathrm{e}-05$

4.873051e-05

$2.363612 \mathrm{e}-02$

$4.815663 \mathrm{e}-02$

$4.549649 \mathrm{e}-02$

$3.990404 \mathrm{e}-02$

$5.663964 \mathrm{e}-02$

$6.445440 \mathrm{e}-02$

$7.031837 \mathrm{e}-02$

$4.326321 \mathrm{e}-02$

$1.027060 \mathrm{e}-02$

$1.181787 \mathrm{e}-01$

5.975898e-02

$6.330292 \mathrm{e}-02$

$3.805883 \mathrm{e}-02$

$4.442856 \mathrm{e}-02$

$2.725811 \mathrm{e}-02$

$4.833551 \mathrm{e}-02$

$3.183634 \mathrm{e}-02$

$1.134561 \mathrm{e}-02$

$1.715366 \mathrm{e}-02$

$3.894664 \mathrm{e}-02$

$1.542590 \mathrm{e}-01$

$1.334888 \mathrm{e}-01$

$2.300805 \mathrm{e}-02$

$5.664429 \mathrm{e}-02$

$3.032684 \mathrm{e}-02$

$1.749351 \mathrm{e}-02$

$1.133040 \mathrm{e}-02$

$1.543382 \mathrm{e}-02$

$4.108985 \mathrm{e}-02$

$5.762264 \mathrm{e}-03$

$7.852879 \mathrm{e}-03$

$5.668678 \mathrm{e}-02$

$2.597550 \mathrm{e}-02$

$2.950044 \mathrm{e}-02$

$1.479146 \mathrm{e}-02$

$1.017635 \mathrm{e}-02$

$1.055455 \mathrm{e}-02$

$1.445382 \mathrm{e}-02$
$\mathrm{NaN}$

$\mathrm{NaN}$

$\mathrm{NaN}$

$\mathrm{NaN}$

$\mathrm{NaN}$

$\mathrm{NaN}$

$\mathrm{NaN}$

$\mathrm{NaN}$

$\mathrm{NaN}$

$\mathrm{NaN}$

$\mathrm{NaN}$

$\mathrm{NaN}$

$\mathrm{NaN}$

$\mathrm{NaN}$

$\mathrm{NaN}$

$\mathrm{NaN}$

$\mathrm{NaN}$

$\mathrm{NaN}$

$\mathrm{NaN}$

$\mathrm{NaN}$

$\mathrm{NaN}$

$\mathrm{NaN}$

$\mathrm{NaN}$

$1.867244 \mathrm{e}-03$

$8.644614 \mathrm{e}-02$

$3.271960 \mathrm{e}-04$

$6.060328 \mathrm{e}-03$

$7.728045 \mathrm{e}-02$

$2.812034 \mathrm{e}-02$

$3.100722 \mathrm{e}-01$

$1.617134 \mathrm{e}-01$

$5.090574 \mathrm{e}-01$

$7.902605 \mathrm{e}-01$

4.712605e-01

$9.288961 \mathrm{e}-01$

9.158825e-01

$8.434375 \mathrm{e}-01$

$7.345137 \mathrm{e}-01$

$5.810366 \mathrm{e}-01$

$1.949588 \mathrm{e}-01$

$6.267030 \mathrm{e}-01$

9.001432e-01

8.301416e-01

$8.740829 \mathrm{e}-01$

$5.030363 \mathrm{e}-01$

$4.326555 \mathrm{e}-01$

3.922026e-01

$9.401199 \mathrm{e}-01$

$1.674428 \mathrm{e}-03$

$6.243952 \mathrm{e}-01$

$7.714923 \mathrm{e}-01$

8.791353e-01

$9.376413 e-01$

$1.968263 \mathrm{e}-02$

7.687890e-01

$1.986273 \mathrm{e}-01$

$4.569939 \mathrm{e}-01$

2.801109e-01

$7.673929 \mathrm{e}-01$

$9.073266 \mathrm{e}-01$

8.396286e-02

$1.331833 \mathrm{e}-01$

3.526502e-01

$1.062335 \mathrm{e}-04$

$9.676323 \mathrm{e}-01$

$1.683220 \mathrm{e}-01$

$7.823404 \mathrm{e}-01$

$9.887460 \mathrm{e}-02$

$4.627820 \mathrm{e}-02$ 
1246 Uncertain_Suggestion Social_science

1247 Uncertain_Suggestion Climatology

1248 Uncertain_Suggestion Pharmacology

1249 Uncertain_Suggestion Agriculture

1250 Uncertain_Suggestion Neuroscience

1251 Uncertain_Suggestion Fishery

1252 Uncertain_Suggestion Virology

1253 Uncertain_Suggestion Microbiology

1254 Uncertain_Suggestion Communication

1255 Uncertain_Suggestion Endocrinology

1256 Uncertain_Suggestion Epigenetics

1257 Uncertain_Suggestion Marketing

1258 Uncertain_Suggestion Global_health

1259 Uncertain_Suggestion Agroforestry

1260 Uncertain_Suggestion Chemistry

1261 Uncertain_Suggestion Mathematics

1262 Uncertain_Suggestion Gynecology

1263 Uncertain_Suggestion Gerontology

1264 Uncertain_Suggestion Meteorology

1265 Uncertain_Suggestion Veterinary_medicine

1266 Uncertain_Suggestion Government

1267 Uncertain_Suggestion Dentistry

1268 Uncertain_Suggestion Microeconomics

1269 Uncertain_Suggestion Sociology

1270 Uncertain_Suggestion Emergency_medicine

1271 Uncertain_Suggestion Pediatrics

1272 Uncertain_Suggestion Advertising

1273 Uncertain_Suggestion Oceanography

1274 Uncertain_Suggestion Developmental_psychology

1275 Uncertain_Suggestion Internal_medicine

1276 Uncertain_Suggestion Cardiology

1277 Uncertain_Suggestion Environmental_engineering

1278 Uncertain_Suggestion General_surgery

1279 Uncertain_Suggestion Environmental_science

1280 Uncertain_Suggestion Geophysics

1281 Uncertain_Suggestion Immunology

1282 Uncertain_Suggestion Clinical_psychology

1283 Uncertain_Suggestion Toxicology

1284 Uncertain_Suggestion Ecology

1285 Uncertain_Suggestion Biology

1286 Uncertain_Suggestion Epidemiology

1287 Uncertain_Suggestion Physical_therapy

1288 Uncertain_Suggestion Physiology

1289 Uncertain_Suggestion Statistics

1290 Uncertain_Suggestion Psychopathology

1291 Uncertain_Suggestion Animal_science

1292 Uncertain_Suggestion Nursing

1293 Uncertain_Suggestion Seismology

1294 Uncertain_Suggestion History

1295 Uncertain_Suggestion Psychiatry

1296 Uncertain_Suggestion Zoology

1297 Uncertain_Suggestion Psychology

1298 Uncertain_Suggestion Etiology

1299 Uncertain_Suggestion Geomorphology

1300 Uncertain_Suggestion Atmospheric_sciences

1301 Uncertain_Suggestion Geography

1302 Uncertain_Suggestion Demography

1303 Uncertain_Suggestion Geology

1304 Uncertain_Suggestion Medicine

1305 Uncertain_Suggestion Family_medicine

1306 Uncertain_Suggestion Evolutionary_biology

1307 Uncertain_Suggestion Astrobiology

1308 Uncertain_Suggestion Astronomy

1309 Uncertain_Suggestion Astrophysics

1310 Uncertain_Suggestion Geochemistry

1311 Uncertain_Suggestion Business

1312 Uncertain_Suggestion Finance

1313 Uncertain_Suggestion Management_science

1314 Uncertain_Suggestion Social_psychology

1315 Uncertain_Suggestion Political_science
$-8.064522 \mathrm{e}-03$

$-7.746988 \mathrm{e}-03$

$-1.154582 \mathrm{e}-02$

$4.581642 \mathrm{e}-02$

$-1.739951 \mathrm{e}-02$

$-7.357160 \mathrm{e}-03$

$-1.025838 \mathrm{e}-02$

$-1.407277 \mathrm{e}-02$

$-7.775990 \mathrm{e}-03$

$3.306488 \mathrm{e}-03$

$-1.170532 \mathrm{e}-02$

$-5.890505 \mathrm{e}-03$

$-1.616995 \mathrm{e}-02$

$-3.457540 \mathrm{e}-02$

$4.551077 \mathrm{e}-02$

$-6.080060 \mathrm{e}-03$

$2.745621 \mathrm{e}-03$

$-8.913656 \mathrm{e}-04$

$-4.081652 \mathrm{e}-03$

$-7.778878 \mathrm{e}-03$

$-1.333452 \mathrm{e}-02$

$-1.935093 \mathrm{e}-02$

$-4.715370 \mathrm{e}-03$

$1.055573 \mathrm{e}-02$

$-3.961473 \mathrm{e}-03$

6.111426e-03

$-2.567259 \mathrm{e}-03$

$-3.292443 \mathrm{e}-03$

$1.077173 \mathrm{e}-02$

$5.978058 \mathrm{e}-03$

$1.268667 \mathrm{e}-02$

$-3.178909 \mathrm{e}-02$

$-8.651048 \mathrm{e}-03$

$5.042320 \mathrm{e}-03$

$-4.891734 \mathrm{e}-03$

$-2.452741 \mathrm{e}-03$

$1.072300 \mathrm{e}-02$

$-2.410973 \mathrm{e}-02$

$-6.095057 \mathrm{e}-03$

$1.154563 \mathrm{e}-02$

$-8.426049 \mathrm{e}-03$

$1.584206 \mathrm{e}-02$

$-5.625106 \mathrm{e}-03$

$5.057500 \mathrm{e}-03$

$-1.015400 \mathrm{e}-02$

$-7.754407 \mathrm{e}-03$

$8.284643 \mathrm{e}-03$

$-8.361010 \mathrm{e}-03$

$1.495849 \mathrm{e}-02$

$1.481884 \mathrm{e}-02$

$-1.115926 \mathrm{e}-02$

2.402052e-02

$-1.341029 \mathrm{e}-02$

$-5.172582 \mathrm{e}-03$

$-2.766515 \mathrm{e}-03$

$-5.384843 \mathrm{e}-04$

$2.255693 \mathrm{e}-03$

$5.191933 \mathrm{e}-03$

$-5.656349 \mathrm{e}-03$

3.107898e-02

$-1.194638 \mathrm{e}-02$

$-9.176464 \mathrm{e}-03$

$-4.942890 \mathrm{e}-03$

$-3.751304 \mathrm{e}-03$

$-4.843630 \mathrm{e}-03$

$-1.435803 \mathrm{e}-03$

$-7.500047 \mathrm{e}-03$

$-1.752187 \mathrm{e}-13$

$-2.547493 \mathrm{e}-02$

4.882161e-03 5.753048e-02

$1.598035 \mathrm{e}-02$

$2.306758 \mathrm{e}-02$

$1.864350 \mathrm{e}-02$

$1.240465 \mathrm{e}-02$

$2.654194 \mathrm{e}-02$

$1.196852 \mathrm{e}-02$

$1.780234 \mathrm{e}-02$

$1.828042 \mathrm{e}-02$

$5.894647 \mathrm{e}-03$

$1.765509 \mathrm{e}-02$

$1.904549 \mathrm{e}-02$

$2.797716 \mathrm{e}-02$

$2.444457 \mathrm{e}-02$

$2.041903 \mathrm{e}-02$

$5.950688 \mathrm{e}-02$

9.762481e-03

$1.034624 \mathrm{e}-02$

$3.093270 \mathrm{e}-02$

$7.061607 \mathrm{e}-02$

$2.076807 \mathrm{e}-02$

$3.828716 \mathrm{e}-02$

$3.254322 \mathrm{e}-02$

4.060861e-02

$8.658169 \mathrm{e}-03$

$6.954654 \mathrm{e}-03$

$1.884721 \mathrm{e}-02$

$2.872492 \mathrm{e}-02$

$1.038488 \mathrm{e}-02$

$5.681371 \mathrm{e}-03$

$9.040379 \mathrm{e}-03$

$2.726129 \mathrm{e}-02$

$1.054332 \mathrm{e}-02$

$2.147556 \mathrm{e}-02$

$5.575528 \mathrm{e}-02$

$7.455186 \mathrm{e}-03$

$1.076169 \mathrm{e}-02$

$3.474156 \mathrm{e}-02$

$8.290536 \mathrm{e}-03$

$1.589396 \mathrm{e}-02$

$8.727219 \mathrm{e}-03$

$5.677519 \mathrm{e}-03$

$2.192097 \mathrm{e}-02$

$7.016649 \mathrm{e}-02$

$2.544476 \mathrm{e}-02$

$3.975088 \mathrm{e}-02$

$1.031156 \mathrm{e}-02$

$4.360468 \mathrm{e}-02$

$1.220081 \mathrm{e}-01$

$7.228549 \mathrm{e}-03$

$1.895842 \mathrm{e}-02$

$1.577253 \mathrm{e}-02$

$2.022104 \mathrm{e}-02$

$2.378683 \mathrm{e}-02$

$2.552268 \mathrm{e}-02$

$2.193638 \mathrm{e}-02$

$6.665442 \mathrm{e}-03$

$2.384814 \mathrm{e}-02$

$1.359069 \mathrm{e}-02$

$6.794802 \mathrm{e}-03$

$1.694751 \mathrm{e}-02$

$3.608456 \mathrm{e}-02$

2.707140e-02

4.175956e-02

$4.053698 \mathrm{e}-02$

$2.314729 \mathrm{e}-02$

$2.452089 \mathrm{e}-02$

$3.931069 \mathrm{e}-13$

$7.937484 \mathrm{e}-03$

3.687643e-02
$8.885213 \mathrm{e}-01$

$6.278389 \mathrm{e}-01$

$6.167163 \mathrm{e}-01$

$1.400364 \mathrm{e}-02$

$1.607419 \mathrm{e}-01$

7.816386e-01

$3.913980 \mathrm{e}-01$

$4.292493 \mathrm{e}-01$

$6.705721 \mathrm{e}-01$

$5.748545 \mathrm{e}-01$

$5.073423 \mathrm{e}-01$

$7.571088 \mathrm{e}-01$

$5.632946 \mathrm{e}-01$

$1.572564 \mathrm{e}-01$

$2.584143 \mathrm{e}-02$

$9.186201 \mathrm{e}-01$

$7.785292 \mathrm{e}-01$

$9.313457 \mathrm{e}-01$

$8.950238 \mathrm{e}-01$

$9.122863 \mathrm{e}-01$

$5.208401 \mathrm{e}-01$

$6.132755 \mathrm{e}-01$

$8.847955 \mathrm{e}-01$

$7.949155 \mathrm{e}-01$

$6.472894 \mathrm{e}-01$

$3.795511 \mathrm{e}-01$

8.916540e-01

9.087483e-01

$2.996384 \mathrm{e}-01$

$2.927176 \mathrm{e}-01$

$1.605410 \mathrm{e}-01$

2.435998e-01

4.119326e-01

8.143727e-01

$9.300880 \mathrm{e}-01$

$7.421625 \mathrm{e}-01$

$3.190724 \mathrm{e}-01$

4.877110e-01

4.622416e-01

4.675968e-01

$3.343170 \mathrm{e}-01$

$5.273329 \mathrm{e}-03$

$7.974851 \mathrm{e}-01$

$9.425405 \mathrm{e}-01$

$6.898552 \mathrm{e}-01$

$8.453372 \mathrm{e}-01$

$4.217394 \mathrm{e}-01$

$8.479444 \mathrm{e}-01$

$9.024238 \mathrm{e}-01$

4.038008e-02

5.561281e-01

$1.277994 \mathrm{e}-01$

$5.072238 \mathrm{e}-01$

$8.278566 \mathrm{e}-01$

$9.136845 \mathrm{e}-01$

9.804162e-01

$7.350551 \mathrm{e}-01$

$8.276600 \mathrm{e}-01$

$6.772756 \mathrm{e}-01$

$4.830554 \mathrm{e}-06$

$4.808822 \mathrm{e}-01$

$7.992644 \mathrm{e}-01$

$8.551248 \mathrm{e}-01$

$9.284229 \mathrm{e}-01$

9.048916e-01

9.505407e-01

$7.597134 \mathrm{e}-01$

$6.558012 \mathrm{e}-01$

$1.333123 \mathrm{e}-03$

8.946759e-01 
1316 Uncertain_Suggestion Archaeology

1317 Uncertain_Suggestion Economics

1318 Uncertain_Suggestion Paleontology

1319 Uncertain_Suggestion Criminology

1320 Uncertain_Framing Intercept

1321 Uncertain_Framing source[T.news]

1322 Uncertain_Framing finding_len

1323 Uncertain_Framing flesch_reading_ease

1324 Uncertain_Framing first_author_rank

1325 Uncertain_Framing

1326 Uncertain_Framing

1327 Uncertain_Framing

1328 Uncertain_Framing

1329 Uncertain_Framing

1330 Uncertain_Framing

1331 Uncertain_Framing

1332 Uncertain_Framing

1333 Uncertain_Framing

1334 Uncertain_Framing

1335 Uncertain_Framing

1336 Uncertain_Framing

1337 Uncertain_Framing

1338 Uncertain_Framing

1339 Uncertain_Framing

1340 Uncertain_Framing

1341 Uncertain_Framing

1342 Uncertain_Framing

1343 Uncertain_Framing

1344 Uncertain_Framing

1345 Uncertain_Framing

1346 Uncertain_Framing

1347 Uncertain_Framing

1348 Uncertain_Framing

1349 Uncertain_Framing

1350 Uncertain_Framing

1351 Uncertain_Framing

1352 Uncertain_Framing

1353 Uncertain_Framing

1354 Uncertain_Framing

1355 Uncertain_Framing

1356 Uncertain_Framing

1357 Uncertain_Framing

1358 Uncertain_Framing

1359 Uncertain_Framing

1360 Uncertain_Framing

1361 Uncertain_Framing

1362 Uncertain_Framing

1363 Uncertain_Framing

1364 Uncertain_Framing

1365 Uncertain_Framing

1366 Uncertain_Framing

1367 Uncertain_Framing

1368 Uncertain_Framing

1369 Uncertain_Framing

1370 Uncertain_Framing

1371 Uncertain_Framing

1372 Uncertain_Framing

1373 Uncertain_Framing

1374 Uncertain_Framing

1375 Uncertain_Framing

1376 Uncertain_Framing

1377 Uncertain_Framing

1378 Uncertain_Framing

1379 Uncertain_Framing

1380 Uncertain_Framing

1381 Uncertain_Framing

1382 Uncertain_Framing

1383 Uncertain_Framing

1384 Uncertain_Framing

Molecular_biology affi_rank

num_authors

journal_impact

Law

Condensed_matter_physics

Biomedical_engineering

Hydrology

Atomic_physics

Development_economics

Optics

Public_policy

Lung_cancer

Electronic_engineering

Artificial_intelligence

Machine_learning

Gender_studies

Chemical_engineering

Biophysics

Analytical_chemistry

Computer_science

Radiology

Urology

Architecture

Quantum_mechanics

Particle_physics

Materials_science

Computational_biology

Agronomy

Environmental_resource management

Dermatology

Engineering

Surgery

Public_health

Molecular_physics

Bioinformatics

Physics

Cognitive_psychology

Oncology

Pathology

Biochemistry

Social_science

Climatology

Pharmacology

Agriculture

Neuroscience

Fishery

Virology

Microbiology

Communication

Endocrinology

Epigenetics

Marketing

Global health

Agroforestry

Chemistry

Mathematics

Gynecology

Gerontology

Meteorology
$-1.840884 \mathrm{e}-03$

$-2.313135 \mathrm{e}-03$

$-1.281670 \mathrm{e}-02$

$-2.036915 \mathrm{e}-02$

8.281496e-02

$-7.710937 \mathrm{e}-03$

$5.739700 \mathrm{e}-04$

$-1.602138 \mathrm{e}-04$

$-1.394150 \mathrm{e}-06$

$-1.812258 \mathrm{e}-06$

$-5.742586 \mathrm{e}-06$

$-3.303234 \mathrm{e}-05$

$1.873384 \mathrm{e}-01$

$-2.862624 \mathrm{e}-01$

$-3.400521 \mathrm{e}-02$

$-3.692180 \mathrm{e}-02$

$-6.035571 \mathrm{e}-02$

$-6.019191 \mathrm{e}-02$

$-6.412809 \mathrm{e}-02$

$-9.074627 \mathrm{e}-02$

7.470580e-02

$-4.896030 \mathrm{e}-02$

$-1.208984 \mathrm{e}-01$

$3.247193 \mathrm{e}-01$

$-3.084225 \mathrm{e}-02$

$-2.761438 \mathrm{e}-02$

$-2.576067 \mathrm{e}-02$

$8.234614 \mathrm{e}-04$

$-1.108400 \mathrm{e}-01$

$-5.880081 \mathrm{e}-02$

$-2.619999 \mathrm{e}-02$

$-4.681033 \mathrm{e}-02$

$-9.729366 \mathrm{e}-02$

$-1.780182 \mathrm{e}-02$

$-9.959486 \mathrm{e}-02$

$-4.683668 \mathrm{e}-02$

$-3.451915 \mathrm{e}-02$

$4.050763 \mathrm{e}-02$

$-3.798837 \mathrm{e}-02$

$-4.477060 \mathrm{e}-03$

$-1.546452 \mathrm{e}-01$

$1.520634 \mathrm{e}-03$

$-2.271512 \mathrm{e}-02$

$9.928907 \mathrm{e}-01$

$-4.748700 \mathrm{e}-02$

$-9.519299 \mathrm{e}-03$

$-1.995946 \mathrm{e}-02$

$3.211170 \mathrm{e}-03$

$-3.439106 \mathrm{e}-02$

$2.179685 \mathrm{e}-02$

$1.348591 \mathrm{e}-02$

9.375349e-03

$-5.408905 \mathrm{e}-02$

$3.850984 \mathrm{e}-02$

$-3.819941 \mathrm{e}-02$

$-5.701099 \mathrm{e}-02$

$-4.021547 \mathrm{e}-03$

$-5.514412 \mathrm{e}-02$

$2.351208 \mathrm{e}-02$

$-2.441709 \mathrm{e}-02$

$-2.447318 \mathrm{e}-02$

$-3.028020 \mathrm{e}-02$

$1.728898 \mathrm{e}-01$

$-6.645924 \mathrm{e}-02$

$-1.130828 \mathrm{e}-01$

$-1.537482 \mathrm{e}-01$

$2.306441 \mathrm{e}-02$

$-3.461012 \mathrm{e}-03$

7.683999e-03
$3.586326 \mathrm{e}-02$

$1.811536 \mathrm{e}-02$

$2.218892 \mathrm{e}-02$

$5.167358 \mathrm{e}-02$

$1.605147 \mathrm{e}-02$

$2.255195 \mathrm{e}-03$

$1.272765 \mathrm{e}-04$

$5.076609 \mathrm{e}-05$

$6.500613 \mathrm{e}-07$

$6.483269 \mathrm{e}-07$

$8.838919 \mathrm{e}-05$

$9.113199 \mathrm{e}-05$

4.420243e-02

$9.005878 \mathrm{e}-02$

$8.508398 \mathrm{e}-02$

7.462543e-02

$1.059230 \mathrm{e}-01$

$1.205376 \mathrm{e}-01$

$1.315039 \mathrm{e}-01$

$8.090749 \mathrm{e}-02$

$1.920728 \mathrm{e}-02$

$2.210086 \mathrm{e}-01$

$1.117566 \mathrm{e}-01$

$1.183842 \mathrm{e}-01$

7.117466e-02

8.308683e-02

$5.097600 \mathrm{e}-02$

9.039331e-02

$5.953785 \mathrm{e}-02$

$2.121768 \mathrm{e}-02$

$3.207945 \mathrm{e}-02$

7.283497e-02

$2.884831 \mathrm{e}-01$

$2.496404 \mathrm{e}-01$

4.302787e-02

$1.059317 \mathrm{e}-01$

$5.671490 \mathrm{e}-02$

$3.271500 \mathrm{e}-02$

$2.118924 \mathrm{e}-02$

$2.886312 \mathrm{e}-02$

$7.684303 \mathrm{e}-02$

$1.077614 \mathrm{e}-02$

$1.468584 \mathrm{e}-02$

$1.060112 \mathrm{e}-01$

$4.857736 \mathrm{e}-02$

5.516942e-02

$2.766183 \mathrm{e}-02$

$1.903102 \mathrm{e}-02$

$1.973829 \mathrm{e}-02$

2.703041e-02

$1.075890 \mathrm{e}-01$

$2.988519 \mathrm{e}-02$

$4.313919 \mathrm{e}-02$

3.486561e-02

$2.319821 \mathrm{e}-02$

$4.963666 \mathrm{e}-02$

$2.238259 \mathrm{e}-02$

$3.329254 \mathrm{e}-02$

$3.418662 \mathrm{e}-02$

$1.102371 \mathrm{e}-02$

3.301716e-02

3.561738e-02

$5.232070 \mathrm{e}-02$

$4.571433 \mathrm{e}-02$

$3.818608 \mathrm{e}-02$

$1.112851 \mathrm{e}-01$

$1.825703 \mathrm{e}-02$

$1.934874 \mathrm{e}-02$

$5.784793 \mathrm{e}-02$

9.590628e-01 $8.983969 \mathrm{e}-01$

$5.635324 \mathrm{e}-01$

$6.934480 \mathrm{e}-01$

$2.514565 \mathrm{e}-07$

6.300010e-04

$6.550709 \mathrm{e}-06$

$1.603560 \mathrm{e}-03$

$3.199970 \mathrm{e}-02$

$5.192976 \mathrm{e}-03$

$9.481994 \mathrm{e}-01$

7.170090e-01

$2.268742 \mathrm{e}-05$

$1.483244 \mathrm{e}-03$

$6.894087 \mathrm{e}-01$

$6.207767 \mathrm{e}-01$

$5.688183 \mathrm{e}-01$

$6.175327 \mathrm{e}-01$

$6.258049 \mathrm{e}-01$

$2.620509 \mathrm{e}-01$

$1.009687 \mathrm{e}-04$

$8.246823 \mathrm{e}-01$ 
1385 Uncertain_Framing

1386 Uncertain_Framing

1387 Uncertain_Framing

1388 Uncertain_Framing

1389 Uncertain_Framing

1390 Uncertain_Framing

1391 Uncertain_Framing

1392 Uncertain_Framing

1393 Uncertain_Framing

1394 Uncertain_Framing

1395 Uncertain_Framing

1396 Uncertain_Framing

1397 Uncertain_Framing

1398 Uncertain_Framing

1399 Uncertain_Framing

1400 Uncertain_Framing

1401 Uncertain_Framing

1402 Uncertain_Framing

1403 Uncertain_Framing

1404 Uncertain_Framing

1405 Uncertain_Framing

1406 Uncertain_Framing

1407 Uncertain_Framing

1408 Uncertain_Framing

1409 Uncertain_Framing

1410 Uncertain_Framing

1411 Uncertain_Framing

1412 Uncertain_Framing

1413 Uncertain_Framing

1414 Uncertain_Framing

1415 Uncertain_Framing

1416 Uncertain_Framing

1417 Uncertain_Framing

1418 Uncertain_Framing

1419 Uncertain_Framing

1420 Uncertain_Framing

1421 Uncertain_Framing

1422 Uncertain_Framing

1423 Uncertain_Framing

1424 Uncertain_Framing

1425 Uncertain_Framing

1426 Uncertain_Framing

1427 Uncertain_Framing

1428 Uncertain_Framing

1429 Uncertain_Framing

1430 Uncertain_Framing

1431 Uncertain_Framing

1432 Uncertain_Framing

1433 Uncertain_Framing

1434 Uncertain_Framing

1435 Uncertain_Framing

1436 Uncertain_Framing

1437 Uncertain_Framing

1438 Uncertain_Framing

1439 Uncertain_Framing
Veterinary_medicine

Government

Dentistry

Microeconomics

Sociology

Emergency_medicine

Pediatrics

Advertising

Oceanography

Developmental_psychology

Internal_medicine

Cardiology

Environmental_engineering

General_surgery

Environmental_science

Geophysics

Immunology

Clinical_psychology

Toxicology

Ecology

Biology

Epidemiology

Physical_therapy

Physiology

Statistics

Psychopathology

Animal_science

Nursing

Seismology

History

Psychiatry

Zoology

Psychology

Etiology

Geomorphology

Atmospheric_sciences

Geography

Demography

Geology

Medicine

Family_medicine

Evolutionary_biology

Astrobiology

Astronomy

Astrophysics

Geochemistry

Business

Finance

Management_science

Social_psychology

Political_science

Archaeology

Economics

Paleontology

Criminology 3.167938e-01

$-2.594031 \mathrm{e}-04$

$2.029945 \mathrm{e}-01$

2.300896e-01

$-1.089801 \mathrm{e}-01$

$-2.438546 \mathrm{e}-02$

$-5.797582 \mathrm{e}-04$

$-3.070616 \mathrm{e}-02$

$1.924933 \mathrm{e}-01$

$-2.028550 \mathrm{e}-02$

$-3.495452 \mathrm{e}-02$

$-1.344618 \mathrm{e}-02$

$-7.050410 \mathrm{e}-02$

$1.948310 \mathrm{e}-02$

$-8.912884 \mathrm{e}-02$

$-1.023038 \mathrm{e}-01$

$-1.522179 \mathrm{e}-02$

$-3.370913 \mathrm{e}-02$

$2.826013 \mathrm{e}-02$

$-4.505954 \mathrm{e}-03$

$-8.572393 \mathrm{e}-02$

$-7.238877 \mathrm{e}-03$

$3.665741 \mathrm{e}-03$

$-4.007166 \mathrm{e}-02$

$-2.529518 \mathrm{e}-03$

5.274026e-02

8.712150e-02

$-4.739154 \mathrm{e}-03$

$-8.340940 \mathrm{e}-02$

$-7.951242 \mathrm{e}-02$

$-1.074816 \mathrm{e}-02$

$7.152264 \mathrm{e}-02$

$-5.413890 \mathrm{e}-02$

$-3.168746 \mathrm{e}-02$

$-3.529849 \mathrm{e}-02$

$-9.218068 \mathrm{e}-02$

$-5.942883 \mathrm{e}-02$

9.239116e-03

$2.528768 \mathrm{e}-02$

$-7.551127 \mathrm{e}-02$

8.199466e-03

$-5.102185 \mathrm{e}-03$

$-1.282909 \mathrm{e}-01$

$1.609425 \mathrm{e}-01$

$-2.128578 \mathrm{e}-01$

$-1.096710 \mathrm{e}-01$

$-1.029544 \mathrm{e}-01$

$-4.962095 \mathrm{e}-02$

$-9.394623 \mathrm{e}-13$

$-2.200380 \mathrm{e}-02$

$-9.999354 \mathrm{e}-02$

$-6.543096 \mathrm{e}-02$

$-3.821832 \mathrm{e}-02$

$-1.831027 \mathrm{e}-02$

$-9.719018 \mathrm{e}-02$
$1.320607 \mathrm{e}-01$

$3.883883 \mathrm{e}-02$

7.160167e-02

$6.085978 \mathrm{e}-02$

$7.594305 \mathrm{e}-02$

$1.619183 \mathrm{e}-02$

$1.300605 \mathrm{e}-02$

$3.524659 \mathrm{e}-02$

$5.371910 \mathrm{e}-02$

$1.942100 \mathrm{e}-02$

$1.062486 \mathrm{e}-02$

$1.690661 \mathrm{e}-02$

$5.098194 \mathrm{e}-02$

$1.971730 \mathrm{e}-02$

$4.016191 \mathrm{e}-02$

$1.042692 \mathrm{e}-01$

$1.394211 \mathrm{e}-02$

$2.012568 \mathrm{e}-02$

$6.497095 \mathrm{e}-02$

$1.550431 \mathrm{e}-02$

$2.972365 \mathrm{e}-02$

$1.632096 \mathrm{e}-02$

$1.061765 \mathrm{e}-02$

4.099489e-02

$1.312199 \mathrm{e}-01$

4.758480e-02

$7.433899 \mathrm{e}-02$

$1.928387 \mathrm{e}-02$

8.154607e-02

2.281701e-01

$1.351827 \mathrm{e}-02$

$3.545456 \mathrm{e}-02$

$2.949655 \mathrm{e}-02$

3.781581e-02

$4.448428 \mathrm{e}-02$

4.773052e-02

4.102371e-02

$1.246519 \mathrm{e}-02$

$4.459893 \mathrm{e}-02$

$2.541624 \mathrm{e}-02$

$1.270711 \mathrm{e}-02$

3.169391e-02

$6.748252 \mathrm{e}-02$

5.062681e-02

$7.809546 \mathrm{e}-02$

$7.580909 \mathrm{e}-02$

$4.328825 \mathrm{e}-02$

$4.585706 \mathrm{e}-02$

$7.351578 \mathrm{e}-13$

$1.484406 \mathrm{e}-02$

$6.896342 \mathrm{e}-02$

$6.706866 \mathrm{e}-02$

$3.387794 \mathrm{e}-02$

4.149598e-02

9.663591e-02
$1.646058 \mathrm{e}-02$

$9.946711 \mathrm{e}-01$

4.588857e-03

$1.571153 \mathrm{e}-04$

$1.513043 \mathrm{e}-01$

$1.320824 \mathrm{e}-01$

$9.644459 \mathrm{e}-01$

$3.836714 \mathrm{e}-01$

3.404772e-04

$2.962671 \mathrm{e}-01$

$1.004953 \mathrm{e}-03$

$4.264415 \mathrm{e}-01$

$1.667121 \mathrm{e}-01$

$3.231113 \mathrm{e}-01$

$2.648766 \mathrm{e}-02$

$3.265367 \mathrm{e}-01$

$2.749477 \mathrm{e}-01$

$9.397174 \mathrm{e}-02$

6.635946e-01

$7.713421 \mathrm{e}-01$

$3.932622 \mathrm{e}-03$

$6.573880 \mathrm{e}-01$

$7.299123 \mathrm{e}-01$

$3.283501 \mathrm{e}-01$

$9.846205 \mathrm{e}-01$

$2.677343 \mathrm{e}-01$

$2.412390 \mathrm{e}-01$

$8.058739 \mathrm{e}-01$

$3.063977 \mathrm{e}-01$

$7.274863 \mathrm{e}-01$

$4.265792 \mathrm{e}-01$

$4.368420 \mathrm{e}-02$

$6.646454 \mathrm{e}-02$

4.020786e-01

4.274981e-01

$5.347090 \mathrm{e}-02$

$1.474605 \mathrm{e}-01$

$4.585895 \mathrm{e}-01$

5.707226e-01

$2.973872 \mathrm{e}-03$

$5.187661 \mathrm{e}-01$

8.721092e-01

$5.731124 \mathrm{e}-02$

$1.481300 \mathrm{e}-03$

$6.426819 \mathrm{e}-03$

$1.480126 \mathrm{e}-01$

$1.740480 \mathrm{e}-02$

$2.792376 \mathrm{e}-01$

$2.013054 \mathrm{e}-01$

$1.382766 \mathrm{e}-01$

$1.470964 \mathrm{e}-01$

$3.292899 \mathrm{e}-01$

$2.592907 \mathrm{e}-01$

$6.590363 \mathrm{e}-01$

$3.145614 \mathrm{e}-01$

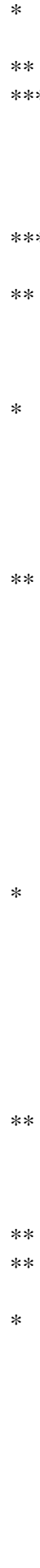

**

Table 7: Regression coefficients for predicting aspect-level certainty with the source of the finding (RQ2). 


\begin{tabular}{|c|c|c|c|c|c|}
\hline & index & coeff & SE & $\mathrm{p}$ value & \\
\hline 0 & Intercept & $1.964574 \mathrm{e}+00$ & $1.730216 \mathrm{e}-01$ & $1.361045 \mathrm{e}-29$ & $* * *$ \\
\hline 1 & abs_Number_STATE[T.Certain] & $2.844180 \mathrm{e}-02$ & $1.696833 \mathrm{e}-02$ & $9.375438 \mathrm{e}-02$ & \\
\hline 2 & abs_Number_STATE[T.Uncertain] & $-6.859408 \mathrm{e}-02$ & $3.020092 \mathrm{e}-02$ & $2.316485 \mathrm{e}-02$ & * \\
\hline 3 & abs_Extent_STATE[T.Certain] & $1.616107 \mathrm{e}-03$ & $1.655072 \mathrm{e}-02$ & $9.222167 \mathrm{e}-01$ & \\
\hline 4 & abs_Extent_STATE[T.Uncertain] & $2.899808 \mathrm{e}-02$ & $2.107222 \mathrm{e}-02$ & $1.688306 \mathrm{e}-01$ & \\
\hline 5 & abs_Probability_STATE[T.Certain] & $7.646274 \mathrm{e}-03$ & $1.911305 \mathrm{e}-02$ & $6.891295 \mathrm{e}-01$ & \\
\hline 6 & abs_Probability_STATE[T.Uncertain] & $-2.260107 \mathrm{e}-02$ & $3.520943 \mathrm{e}-02$ & $5.209589 \mathrm{e}-01$ & \\
\hline 7 & abs_Condition_STATE[T.Certain] & $-3.411162 \mathrm{e}-02$ & $3.671247 \mathrm{e}-02$ & $3.528436 \mathrm{e}-01$ & \\
\hline 8 & abs_Suggestion_STATE[T.Certain] & $-1.400909 \mathrm{e}-01$ & $4.805333 \mathrm{e}-02$ & $3.565707 \mathrm{e}-03$ & ** \\
\hline 9 & abs_Suggestion_STATE[T.Uncertain] & $-6.587783 e-02$ & $9.853438 \mathrm{e}-02$ & $5.037897 \mathrm{e}-01$ & \\
\hline 10 & abs_Framing_STATE[T.Certain] & $3.585483 \mathrm{e}-03$ & $2.031451 \mathrm{e}-02$ & $8.599079 \mathrm{e}-01$ & \\
\hline 11 & abs_Framing_STATE[T.Uncertain] & $-1.076960 \mathrm{e}-01$ & $4.333388 \mathrm{e}-02$ & $1.297133 \mathrm{e}-02$ & * \\
\hline 12 & journal_impact_cat[T.1] & $-9.913563 e-03$ & $9.633521 \mathrm{e}-02$ & $9.180401 \mathrm{e}-01$ & \\
\hline 13 & journal_impact_cat[T.10] & $8.967768 \mathrm{e}-02$ & $1.000680 \mathrm{e}-01$ & $3.701977 \mathrm{e}-01$ & \\
\hline 14 & journal_impact_cat[T.11-20] & $1.451402 \mathrm{e}-02$ & $9.405024 \mathrm{e}-02$ & $8.773608 \mathrm{e}-01$ & \\
\hline 15 & journal_impact_cat[T.2] & $-1.059573 \mathrm{e}-02$ & $9.449466 \mathrm{e}-02$ & $9.107235 \mathrm{e}-01$ & \\
\hline 16 & journal_impact_cat[T.21-30] & $9.153368 \mathrm{e}-03$ & $9.835565 \mathrm{e}-02$ & $9.258557 \mathrm{e}-01$ & \\
\hline 17 & journal_impact_cat[T.3] & $-2.676568 \mathrm{e}-03$ & $9.438566 \mathrm{e}-02$ & $9.773777 \mathrm{e}-01$ & \\
\hline 18 & journal_impact_cat[T.4] & $1.233059 \mathrm{e}-02$ & $9.435727 \mathrm{e}-02$ & $8.960328 \mathrm{e}-01$ & \\
\hline 19 & journal_impact_cat[T.5] & $6.302687 \mathrm{e}-02$ & $9.501415 \mathrm{e}-02$ & $5.071358 \mathrm{e}-01$ & \\
\hline 20 & journal_impact_cat[T.6] & $-9.899351 \mathrm{e}-03$ & $9.781021 \mathrm{e}-02$ & $9.193871 \mathrm{e}-01$ & \\
\hline 21 & journal_impact_cat[T.7] & $2.351605 \mathrm{e}-02$ & $9.806796 \mathrm{e}-02$ & $8.104982 \mathrm{e}-01$ & \\
\hline 22 & journal_impact_cat[T.8] & $-8.979248 \mathrm{e}-03$ & $9.976485 \mathrm{e}-02$ & $9.282868 \mathrm{e}-01$ & \\
\hline 23 & journal_impact_cat[T.9] & $7.005939 \mathrm{e}-02$ & $9.561894 \mathrm{e}-02$ & $4.637724 \mathrm{e}-01$ & \\
\hline 24 & journal_impact_cat[T.>30] & $7.395042 \mathrm{e}-02$ & $9.522755 \mathrm{e}-02$ & $4.374443 \mathrm{e}-01$ & \\
\hline 25 & num_authors_cat[T.10] & $3.737626 \mathrm{e}-02$ & $5.971992 \mathrm{e}-02$ & $5.314299 \mathrm{e}-01$ & \\
\hline 26 & num_authors_cat[T.11-20] & $4.302108 \mathrm{e}-02$ & $5.528899 \mathrm{e}-02$ & $4.365315 \mathrm{e}-01$ & \\
\hline 27 & num_authors_cat[T.2] & $-3.563177 \mathrm{e}-02$ & $5.627319 \mathrm{e}-02$ & $5.266308 \mathrm{e}-01$ & \\
\hline 28 & num_authors_cat[T.21-30] & $-4.554514 \mathrm{e}-02$ & $6.275255 \mathrm{e}-02$ & $4.679948 \mathrm{e}-01$ & \\
\hline 29 & num_authors_cat[T.3] & $3.064185 \mathrm{e}-02$ & $5.540858 \mathrm{e}-02$ & $5.802718 \mathrm{e}-01$ & \\
\hline 30 & num_authors_cat[T.4] & $1.747497 \mathrm{e}-02$ & $5.499067 \mathrm{e}-02$ & $7.506618 \mathrm{e}-01$ & \\
\hline 31 & num_authors_cat[T.5] & $4.730588 \mathrm{e}-02$ & $5.508287 \mathrm{e}-02$ & $3.904764 \mathrm{e}-01$ & \\
\hline 32 & num_authors_cat[T.6] & $5.416085 \mathrm{e}-02$ & $5.572517 \mathrm{e}-02$ & $3.311237 \mathrm{e}-01$ & \\
\hline 33 & num_authors_cat[T.7] & $1.897214 \mathrm{e}-02$ & $5.632460 \mathrm{e}-02$ & $7.362519 \mathrm{e}-01$ & \\
\hline 34 & num_authors_cat[T.8] & $6.379646 \mathrm{e}-02$ & $5.743058 \mathrm{e}-02$ & $2.666775 \mathrm{e}-01$ & \\
\hline 35 & num_authors_cat[T.9] & $4.382665 \mathrm{e}-02$ & $5.966418 \mathrm{e}-02$ & $4.626375 \mathrm{e}-01$ & \\
\hline 36 & num_authors_cat[T.>30] & $4.471737 \mathrm{e}-03$ & $6.450590 \mathrm{e}-02$ & $9.447348 \mathrm{e}-01$ & \\
\hline 37 & outlet_re[T.7th Space Family Portal] & $-7.314486 \mathrm{e}-02$ & $1.028863 \mathrm{e}-01$ & $4.771545 \mathrm{e}-01$ & \\
\hline 38 & outlet_re[T.ABC News] & $3.046019 \mathrm{e}-01$ & $2.877835 \mathrm{e}-01$ & $2.898947 \mathrm{e}-01$ & \\
\hline 39 & outlet_re[T.AJMC] & $-8.163920 \mathrm{e}-02$ & $1.136995 \mathrm{e}-01$ & $4.727677 \mathrm{e}-01$ & \\
\hline 40 & outlet_re[T.Action News Now] & $-7.902238 \mathrm{e}-02$ & $2.059369 \mathrm{e}-01$ & $7.011979 \mathrm{e}-01$ & \\
\hline 41 & outlet_re[T.Arstechnica] & $-2.216913 e-01$ & $2.067283 e-01$ & $2.835904 \mathrm{e}-01$ & \\
\hline 42 & outlet_re[T.Becker's Hospital Review] & $-1.479676 \mathrm{e}-01$ & $1.556804 \mathrm{e}-01$ & $3.419163 e-01$ & \\
\hline 43 & outlet_re[T.Benzinga] & $-2.235247 \mathrm{e}-01$ & $1.125087 \mathrm{e}-01$ & $4.699517 \mathrm{e}-02$ & * \\
\hline 44 & outlet_re[T.Bio-Medicine.org] & $-4.802014 \mathrm{e}-02$ & $1.485274 \mathrm{e}-01$ & $7.464725 \mathrm{e}-01$ & \\
\hline 45 & outlet_re[T.Biospace] & $5.209157 \mathrm{e}-02$ & $1.548434 \mathrm{e}-01$ & $7.365694 \mathrm{e}-01$ & \\
\hline 46 & outlet_re[T.Business Insider] & $-8.346022 \mathrm{e}-02$ & $1.172547 \mathrm{e}-01$ & $4.766236 \mathrm{e}-01$ & \\
\hline 47 & outlet_re[T.Business Wire] & $1.390192 \mathrm{e}-01$ & $2.464933 \mathrm{e}-01$ & $5.727825 \mathrm{e}-01$ & \\
\hline 48 & outlet_re[T.Bustle] & $-3.241497 \mathrm{e}-01$ & $1.415584 \mathrm{e}-01$ & $2.206187 \mathrm{e}-02$ & * \\
\hline 49 & outlet_re[T.CBS News] & $-1.613810 \mathrm{e}-01$ & $1.541787 \mathrm{e}-01$ & $2.952716 \mathrm{e}-01$ & \\
\hline 50 & outlet_re[T.Christian Science Monitor] & $-2.259828 \mathrm{e}-01$ & $1.684575 \mathrm{e}-01$ & $1.798119 \mathrm{e}-01$ & \\
\hline 51 & outlet_re[T.Clinical Advisor] & $-2.887507 \mathrm{e}-02$ & $9.300493 e-02$ & $7.562152 \mathrm{e}-01$ & \\
\hline 52 & outlet_re[T.Cornell Chronicle] & $-2.067643 e-01$ & $1.498743 \mathrm{e}-01$ & $1.677633 \mathrm{e}-01$ & \\
\hline 53 & outlet_re[T.Counsel \& Heal ] & $2.683303 \mathrm{e}-01$ & $1.210705 \mathrm{e}-01$ & $2.670550 \mathrm{e}-02$ & $*$ \\
\hline 54 & outlet_re[T.Doctors Lounge] & $3.588042 \mathrm{e}-02$ & $7.421485 \mathrm{e}-02$ & $6.287810 \mathrm{e}-01$ & \\
\hline 55 & $\begin{array}{l}\text { outlet_re[T.Drug Discovery and Devel- } \\
\text { opment ] }\end{array}$ & $1.056507 \mathrm{e}-02$ & $1.546392 \mathrm{e}-01$ & $9.455325 \mathrm{e}-01$ & \\
\hline 56 & outlet_re[T.Drugs.com] & $3.059068 \mathrm{e}-02$ & $7.217873 \mathrm{e}-02$ & $6.717126 \mathrm{e}-01$ & \\
\hline 57 & outlet_re[T.ECN] & $-1.527935 \mathrm{e}-01$ & $2.164670 \mathrm{e}-01$ & $4.803066 \mathrm{e}-01$ & \\
\hline 58 & outlet_re[T.EarthSky] & $-1.206184 \mathrm{e}-01$ & $1.762651 \mathrm{e}-01$ & $4.938100 \mathrm{e}-01$ & \\
\hline 59 & outlet_re[T.Elite Daily] & $-1.556170 \mathrm{e}-01$ & $1.943854 \mathrm{e}-01$ & $4.234171 \mathrm{e}-01$ & \\
\hline 60 & outlet_re[T.Emaxhealth.com] & $5.559788 \mathrm{e}-02$ & $1.081245 \mathrm{e}-01$ & $6.071285 \mathrm{e}-01$ & \\
\hline 61 & $\begin{array}{l}\text { outlet_re[T.Environmental News Net- } \\
\text { work] }\end{array}$ & $-3.303157 \mathrm{e}-02$ & $1.327056 \mathrm{e}-01$ & $8.034395 \mathrm{e}-01$ & \\
\hline 62 & outlet_re[T.Everyday Health] & $8.934230 \mathrm{e}-02$ & $2.229055 \mathrm{e}-01$ & $6.885750 \mathrm{e}-01$ & \\
\hline 63 & outlet_re[T.Fight Aging!] & $9.835234 \mathrm{e}-02$ & $1.110734 \mathrm{e}-01$ & $3.759359 \mathrm{e}-01$ & \\
\hline 64 & outlet_re[T.Futurity] & $-4.061382 \mathrm{e}-02$ & $8.267862 \mathrm{e}-02$ & $6.232842 \mathrm{e}-01$ & \\
\hline
\end{tabular}




\begin{tabular}{|c|c|}
\hline 65 & outlet_re[T.GEN] \\
\hline 66 & outlet_re[T.Google News] \\
\hline 67 & outlet_re[T.Green Car Congress] \\
\hline 68 & outlet_re[T.Guardian Liberty Voice] \\
\hline 69 & outlet_re[T.Headlines \& Global News] \\
\hline 70 & outlet_re[T.Health Canal] \\
\hline 71 & outlet_re[T.Health Day ] \\
\hline 72 & outlet_re[T.Health Medicinet] \\
\hline 73 & $\begin{array}{l}\text { outlet_re[T.Homeland Security News } \\
\text { Wire] }\end{array}$ \\
\hline 74 & outlet_re[T.HowStuffWorks] \\
\hline 75 & outlet_re[T.Infection Control Today] \\
\hline 76 & outlet_re[T.Insurance News Net] \\
\hline 77 & $\begin{array}{l}\text { outlet_re[T.International } \\
\text { Times] }\end{array}$ \\
\hline 78 & outlet_re[T.Inverse] \\
\hline 79 & outlet_re[T.KPBS] \\
\hline 80 & outlet_re[T.LiveScience] \\
\hline 81 & outlet_re[T.MIT News] \\
\hline 82 & outlet_re[T.MSN] \\
\hline 83 & outlet_re[T.Medical Daily] \\
\hline 84 & outlet_re[T.Medicinenet] \\
\hline 85 & outlet_re[T.MinnPost] \\
\hline 86 & outlet_re[T.Mongabay] \\
\hline 87 & outlet_re[T.Mother Nature Network] \\
\hline 88 & outlet_re[T.My Science] \\
\hline 89 & outlet_re[T.Nanowerk] \\
\hline 90 & outlet_re[T.National Geographic] \\
\hline 91 & outlet_re[T.Nature World News] \\
\hline 92 & outlet_re[T.Newsweek] \\
\hline 93 & outlet_re[T.Newswise] \\
\hline 94 & outlet_re[T.Nutra Ingredients USA] \\
\hline 95 & outlet_re[T.OnMedica] \\
\hline 96 & outlet_re[T.Oncology Nurse Advisor ] \\
\hline 97 & $\begin{array}{l}\text { outlet_re[T.Oregon Public Broadcast- } \\
\text { ing] }\end{array}$ \\
\hline 98 & outlet_re[T.PR Newswire] \\
\hline 99 & outlet_re[T.PR Web] \\
\hline 100 & outlet_re[T.Pacific Standard] \\
\hline 101 & outlet_re[T.Parent Herald] \\
\hline 102 & outlet_re[T.Pediatric News ] \\
\hline 103 & outlet_re[T.Physician's Briefing] \\
\hline 104 & outlet_re[T.Physician’s Weekly] \\
\hline 105 & outlet_re[T.Psych Central] \\
\hline 106 & outlet_re[T.Psychology Today] \\
\hline 107 & outlet_re[T.Quartz] \\
\hline 108 & outlet_re[T.Real Clear Science] \\
\hline 109 & outlet_re[T.Renal \& Urology News] \\
\hline 110 & outlet_re[T.Runner’s World] \\
\hline 111 & outlet_re[T.Salon] \\
\hline 112 & outlet_re[T.Sci-News] \\
\hline 113 & outlet_re[T.Science 2.0] \\
\hline 114 & outlet_re[T.Science Alert] \\
\hline 115 & outlet_re[T.Science Daily] \\
\hline 116 & outlet_re[T.Science World Report] \\
\hline 117 & outlet_re[T.Science/AAAS] \\
\hline 118 & outlet_re[T.Scientific American] \\
\hline 119 & outlet_re[T.Seed Daily] \\
\hline 120 & outlet_re[T.SeedQuest] \\
\hline 121 & outlet_re[T.Sign of the Times] \\
\hline 122 & outlet_re[T.Sky Nightly ] \\
\hline 123 & outlet_re[T.Smithsonian Magazine] \\
\hline 124 & outlet_re[T.Space Daily] \\
\hline 125 & outlet_re[T.Space.com] \\
\hline 126 & outlet_re[T.TIME Magazine] \\
\hline 127 & outlet_re[T.Tech Times] \\
\hline 128 & outlet_re[T.Tech Xplore] \\
\hline 129 & outlet_re[T.Technology Networks] \\
\hline 130 & outlet_re[T.Technology.org] \\
\hline 131 & outlet_re[T.The ASCO Post] \\
\hline
\end{tabular}

$5.703374 \mathrm{e}-03$

$-4.522779 \mathrm{e}-01$

$6.236724 \mathrm{e}-02$

$2.809698 \mathrm{e}-01$

$1.490881 \mathrm{e}-02$

$-4.314973 \mathrm{e}-02$

$-1.541944 \mathrm{e}-01$

$-8.468564 \mathrm{e}-02$

$-7.181731 \mathrm{e}-02$

$-2.498181 \mathrm{e}-01$

$2.871228 \mathrm{e}-02$

$-6.180401 \mathrm{e}-02$

$-1.627059 \mathrm{e}-01$

$-4.928470 \mathrm{e}-02$

$-4.593463 \mathrm{e}-01$

$-1.306514 \mathrm{e}-01$

$-7.107759 \mathrm{e}-02$

$5.947856 \mathrm{e}-02$

$2.713372 \mathrm{e}-03$

$-8.076063 e-02$

$3.443965 \mathrm{e}-01$

$-6.811923 \mathrm{e}-02$

$-2.763805 \mathrm{e}-01$

3.483963e-01

$8.096455 \mathrm{e}-03$

$-3.036850 \mathrm{e}-01$

$-3.571103 \mathrm{e}-03$

$-1.750058 \mathrm{e}-02$

$-1.369415 \mathrm{e}-01$

5.507245e-02

$-3.932312 \mathrm{e}-01$

$-2.845357 \mathrm{e}-02$

$-2.666921 \mathrm{e}-01$

$-3.164633 \mathrm{e}-01$

$-1.157196 \mathrm{e}-01$

$-7.247648 \mathrm{e}-02$

$-1.371882 \mathrm{e}-02$

$-1.041879 \mathrm{e}-01$

$5.903768 \mathrm{e}-02$

$5.279796 \mathrm{e}-02$

$-7.079623 \mathrm{e}-02$

$1.471616 \mathrm{e}-01$

$-2.568594 \mathrm{e}-02$

$-1.033074 \mathrm{e}-01$

$-2.863998 \mathrm{e}-01$

$1.692774 \mathrm{e}-01$

$-2.017878 \mathrm{e}-01$

$-8.138982 \mathrm{e}-02$

$-1.136839 \mathrm{e}-02$

$-2.144484 \mathrm{e}-01$

$-4.366435 \mathrm{e}-02$

$-1.422420 \mathrm{e}-01$

$-5.761539 \mathrm{e}-02$

$-4.941122 \mathrm{e}-02$

$-1.668183 \mathrm{e}-02$

$-5.266943 \mathrm{e}-02$

$-3.243128 \mathrm{e}-01$

$-2.064380 \mathrm{e}-01$

$-1.020489 \mathrm{e}-01$

$-3.221622 \mathrm{e}-02$

$-2.475331 \mathrm{e}-01$

$-1.573875 \mathrm{e}-01$

$-2.266114 \mathrm{e}-01$

$-1.922758 \mathrm{e}-01$

$1.374658 \mathrm{e}-02$

$-4.991141 \mathrm{e}-02$

$1.168462 \mathrm{e}-02$
$9.537530 \mathrm{e}-02$ $2.486946 \mathrm{e}-01$

$1.123859 \mathrm{e}-01$

$1.495827 \mathrm{e}-01$

$1.082392 \mathrm{e}-01$

$7.433104 \mathrm{e}-02$

$1.402554 \mathrm{e}-01$

7.360682e-02

$1.294845 \mathrm{e}-01$

$2.235940 \mathrm{e}-01$

$1.055070 \mathrm{e}-01$

2.486693e- 01

$1.415930 \mathrm{e}-01$

$8.687417 \mathrm{e}-02$

$4.778852 \mathrm{e}-01$

$1.359060 \mathrm{e}-01$

$1.575200 \mathrm{e}-01$

$1.104433 \mathrm{e}-01$

$8.768741 \mathrm{e}-02$

$1.192707 \mathrm{e}-01$

$1.960939 \mathrm{e}-01$

$1.873440 \mathrm{e}-01$

$1.673802 \mathrm{e}-01$

$1.451694 \mathrm{e}-01$

$1.058460 \mathrm{e}-01$

$2.092475 \mathrm{e}-01$

$9.292002 \mathrm{e}-02$

$1.246192 \mathrm{e}-01$

7.427771e-02

$1.368482 \mathrm{e}-01$

$1.924101 \mathrm{e}-01$

8.021973e-02

$3.417858 \mathrm{e}-01$

$1.351765 \mathrm{e}-01$ $1.834363 \mathrm{e}-01$ $3.525606 \mathrm{e}-01$

1.318641e-01 $1.289190 \mathrm{e}-01$ 7.128629e-02 $8.979990 \mathrm{e}-02$ 8.881198e-02 $1.458590 \mathrm{e}-01$ $1.736216 \mathrm{e}-01$ $2.967537 \mathrm{e}-01$ $1.486517 \mathrm{e}-01$ $1.487845 \mathrm{e}-01$ $1.607046 \mathrm{e}-01$ $1.000133 \mathrm{e}-01$ $8.991745 \mathrm{e}-02$ $1.502139 \mathrm{e}-01$ 7.140627e-02 8.674987e-02 $2.084667 \mathrm{e}-01$ $1.312369 \mathrm{e}-01$ $1.344291 \mathrm{e}-01$ $1.476495 \mathrm{e}-01$ $2.089051 \mathrm{e}-0$ $1.565965 \mathrm{e}-01$ $2.075115 \mathrm{e}-01$ $1.663509 \mathrm{e}-01$ 2.977834e-01 $1.272313 \mathrm{e}-01$ 9.318396e-02 1.704491e-01 8.413666e-02 7.621141e-02 $9.385778 \mathrm{e}-02$ 9.523174e- 01 $6.901864 \mathrm{e}-02$ 5.789564e- 01 $6.037817 \mathrm{e}-02$ 8.904507e-01 5.615931e-01 2.716430e- 01 $2.499760 \mathrm{e}-01$ 5.791603e-01

2.639153e-01 $7.855262 \mathrm{e}-01$ $8.037255 \mathrm{e}-01$ $2.505540 \mathrm{e}-01$

$5.705228 \mathrm{e}-01$ $3.364851 \mathrm{e}-01$ 3.364197e-01 $6.518399 \mathrm{e}-01$ $5.902205 \mathrm{e}-01$ 9.753154e-01 $4.983543 \mathrm{e}-01$

7.908836e-02

7.161651e-01

9.874458e-02

$1.642704 \mathrm{e}-02$ $9.390295 \mathrm{e}-01$

$1.467406 \mathrm{e}-01$ $9.693445 \mathrm{e}-01$ 8.883228e-01 $6.528206 \mathrm{e}-02$ $6.873779 \mathrm{e}-01$ $4.102325 \mathrm{e}-02$ 7.228296e-01 $4.352495 \mathrm{e}-01$

$1.925733 \mathrm{e}-02$ 5.281657e-01 8.371321e-01 9.171429e-01 $4.190258 \mathrm{e}-01$ $4.076014 \mathrm{e}-01$ 5.565861e-01 4.253955e- 01 3.130466e-01 8.823932e-01 $7.277578 \mathrm{e}-01$ $5.406808 \mathrm{e}-02$ $2.552741 \mathrm{e}-01$ $2.092913 \mathrm{e}-01$ $4.157961 \mathrm{e}-01$ 8.993944e-01 $1.534506 \mathrm{e}-01$ $5.408960 \mathrm{e}-01$ $1.011216 \mathrm{e}-01$ 7.822676e-01 $7.065549 \mathrm{e}-01$ 9.012449e-01 $7.213138 \mathrm{e}-01$ $1.206069 \mathrm{e}-01$ $1.874577 \mathrm{e}-01$ 6.228957e- 01 8.464450e- 01 4.058626e-01 2.161261e-01 $1.504862 \mathrm{e}-02$ 2.593397e-01 8.702213e-01 5.125513e-01 9.009290e-01 
outlet_re[T.The Atlanta Journal Constitution]

outlet_re[T.The Atlantic] outlet_re[T.The Epoch Times] outlet_re[T.The Inquisitr] outlet_re[T.The Raw Story] outlet_re[T.The University Herald] outlet_re[T.UBM Medica] outlet_re[T.UPI.com] outlet_re[T.USNews.com] outlet_re[T.Vice] outlet_re[T.Vox.com] outlet_re[T.WebMD News] outlet_re[T.Yahoo!] outlet_re[T.Yahoo! Finance USA] outlet_re[T.newsmax.com] outlet_re[T.outlet_ref] outlet_re[T.redOrbit] abs_level_of_uncertainty abs_finding_len abs_flesch_reading_ease first_author_rank affi_rank

Law

Condensed_matter_physics Biomedical_engineering Hydrology

Atomic_physics

Development_economics Optics

Public_policy

Lung_cancer

Electronic_engineering

Artificial_intelligence

Machine_learning

Gender_studies

Chemical_engineering

Biophysics

Analytical_chemistry

Computer_science

Radiology

Urology

Architecture

Quantum_mechanics

Particle_physics

Materials_science

Computational_biology

Agronomy

Environmental_resource_management Molecular_biology

Dermatology

Engineering

Surgery

Public_health

Molecular_physics

Bioinformatics

Physics

Cognitive_psychology

Oncology

Pathology

Biochemistry

Social_science

Climatology

Pharmacology

Agriculture

Neuroscience

Fishery

Virology

Microbiology

Communication 2.579170e-01

$-8.017929 \mathrm{e}-02$

$-1.312479 \mathrm{e}-01$

$1.302168 \mathrm{e}-01$

$-2.481762 \mathrm{e}-01$

$-6.434642 \mathrm{e}-01$

$-6.016875 \mathrm{e}-02$

8.112067e-02

$-9.798014 \mathrm{e}-02$

$-7.818448 \mathrm{e}-03$

$-3.341810 \mathrm{e}-01$

$-1.310441 \mathrm{e}-01$

$-5.646596 \mathrm{e}-02$

$-7.182726 \mathrm{e}-02$

$1.473574 \mathrm{e}-01$

$-1.387991 \mathrm{e}-01$

$-1.253200 \mathrm{e}-01$

5.699637e-01

$-5.525056 \mathrm{e}-04$

$-4.160216 \mathrm{e}-04$

$-4.919522 \mathrm{e}-06$

$6.934040 \mathrm{e}-06$

7.366396e-01

8.138790e-01

$1.787057 \mathrm{e}-01$

$4.387856 \mathrm{e}-01$

$1.055620 \mathrm{e}+00$

$9.766939 \mathrm{e}-01$

$1.977795 \mathrm{e}-01$

$1.715567 \mathrm{e}-01$

$-1.895581 \mathrm{e}-01$

$7.334788 \mathrm{e}-01$

$4.496298 \mathrm{e}-01$

$1.193459 \mathrm{e}+00$

$1.698486 \mathrm{e}-01$

$1.356124 \mathrm{e}-01$

$-6.251133 \mathrm{e}-01$

$6.470635 \mathrm{e}-02$

$5.993530 \mathrm{e}-01$

$3.306108 \mathrm{e}-01$

$5.536116 \mathrm{e}-01$

7.057613e-01

$-1.200196 \mathrm{e}+00$

$-1.005499 \mathrm{e}+00$

$-1.936561 \mathrm{e}-02$

$1.604343 \mathrm{e}-01$

$-1.691026 \mathrm{e}-01$

$3.442745 \mathrm{e}-01$

$3.329921 \mathrm{e}-01$

$2.053528 \mathrm{e}-01$

$-2.612870 \mathrm{e}-02$

$7.256831 \mathrm{e}-02$

$3.683271 \mathrm{e}-02$

$-2.779245 \mathrm{e}+00$

$3.118113 \mathrm{e}-01$

6.081841e-01

$1.008165 \mathrm{e}-01$

2.514013e-01

$-9.373196 \mathrm{e}-02$

$1.774480 \mathrm{e}-01$

$-6.793392 \mathrm{e}-01$

$-2.748547 \mathrm{e}-02$

$-1.151915 \mathrm{e}-02$

$-1.438755 \mathrm{e}-01$

$2.077526 \mathrm{e}-01$

$-6.280234 \mathrm{e}-02$

$8.153155 \mathrm{e}-02$

$2.829945 \mathrm{e}-01$

$-5.512068 \mathrm{e}-02$ 2.061377e-01

2.251770e-01

$1.110176 \mathrm{e}-01$

$1.825987 \mathrm{e}-01$

$2.229263 \mathrm{e}-01$

$1.599709 \mathrm{e}-01$

$8.026344 \mathrm{e}-02$

$1.267747 \mathrm{e}-01$

$9.177399 \mathrm{e}-02$

$2.517404 \mathrm{e}-01$

$1.929045 \mathrm{e}-01$

$1.952281 \mathrm{e}-01$

$1.108680 \mathrm{e}-01$

$1.125804 \mathrm{e}-01$

$1.651354 \mathrm{e}-01$

$7.826739 \mathrm{e}-02$

$1.020059 \mathrm{e}-01$

$1.522749 \mathrm{e}-02$

$7.162267 \mathrm{e}-04$

$2.800400 \mathrm{e}-04$

$3.511413 \mathrm{e}-06$

$3.471540 \mathrm{e}-06$

$2.361478 \mathrm{e}-01$

$4.870948 \mathrm{e}-01$

$4.496644 \mathrm{e}-01$

$3.984513 \mathrm{e}-01$

$5.598327 \mathrm{e}-01$

$6.344526 \mathrm{e}-01$

6.850613e-01

$4.336509 \mathrm{e}-01$

$1.011566 \mathrm{e}-01$

$1.202981 \mathrm{e}+00$

$5.821348 \mathrm{e}-01$

6.224831e-01

3.783571e-01

$4.603929 \mathrm{e}-01$

2.699860e-01

$4.763130 \mathrm{e}-01$

3.165207e-01

$1.257341 \mathrm{e}-01$

$1.734635 \mathrm{e}-01$

$3.804322 \mathrm{e}-01$

$1.516187 \mathrm{e}+00$

$1.297896 \mathrm{e}+00$

$2.358550 \mathrm{e}-01$

$6.447472 \mathrm{e}-01$

$2.998099 \mathrm{e}-01$

$1.769106 \mathrm{e}-01$

$1.140334 \mathrm{e}-01$

$1.618487 \mathrm{e}-01$

4.064867e-01

$5.796134 \mathrm{e}-02$

$7.998789 \mathrm{e}-02$

$5.568305 \mathrm{e}-01$

$2.547929 \mathrm{e}-01$

$2.931849 \mathrm{e}-01$

$1.476448 \mathrm{e}-01$

$1.018363 \mathrm{e}-01$

$1.049929 \mathrm{e}-01$

$1.429166 \mathrm{e}-01$

5.846966e-01

$1.599616 \mathrm{e}-01$

2.262672e-01

$1.853908 \mathrm{e}-01$

$1.225342 \mathrm{e}-01$

2.614855e-01

$1.191987 \mathrm{e}-01$

$1.755445 \mathrm{e}-01$

$1.818739 \mathrm{e}-01$

2.109122e-01

7.217983e-01

$2.371604 \mathrm{e}-01$

4.757910e-01

$2.656367 \mathrm{e}-01$

$5.828394 \mathrm{e}-05$

$4.534990 \mathrm{e}-01$

5.222736e- 01

$2.857308 \mathrm{e}-01$

9.752246e-01

$8.325744 \mathrm{e}-02$

5.020949e-01

6.105541e-01 


\begin{tabular}{|c|c|c|c|c|c|}
\hline 201 & Endocrinology & $-1.144640 \mathrm{e}-02$ & $6.000891 \mathrm{e}-02$ & $8.487315 \mathrm{e}-01$ & \\
\hline 202 & Epigenetics & $-3.201931 \mathrm{e}-01$ & $1.739367 \mathrm{e}-01$ & $6.568889 \mathrm{e}-02$ & \\
\hline 203 & Marketing & $-5.428802 \mathrm{e}-02$ & $1.878822 \mathrm{e}-01$ & $7.726313 \mathrm{e}-01$ & \\
\hline 204 & Global_health & $-8.158797 \mathrm{e}-01$ & $2.747229 \mathrm{e}-01$ & $2.990865 \mathrm{e}-03$ & $* *$ \\
\hline 205 & Agroforestry & $3.464929 \mathrm{e}-01$ & $2.411601 \mathrm{e}-01$ & $1.508313 \mathrm{e}-01$ & \\
\hline 206 & Chemistry & $-2.867175 e-01$ & $2.035011 \mathrm{e}-01$ & $1.589068 \mathrm{e}-01$ & \\
\hline 207 & Mathematics & $-2.450289 \mathrm{e}-04$ & $5.875854 \mathrm{e}-01$ & $9.996673 \mathrm{e}-01$ & \\
\hline 208 & Gynecology & $5.374073 \mathrm{e}-02$ & $1.003932 \mathrm{e}-01$ & $5.924596 \mathrm{e}-01$ & \\
\hline 209 & Gerontology & $1.067810 \mathrm{e}-01$ & $1.025651 \mathrm{e}-01$ & $2.978669 \mathrm{e}-01$ & \\
\hline 210 & Meteorology & $1.868873 \mathrm{e}-01$ & $3.175428 \mathrm{e}-01$ & $5.561894 \mathrm{e}-01$ & \\
\hline 211 & Veterinary_medicine & $4.891855 \mathrm{e}-01$ & $6.890164 \mathrm{e}-01$ & $4.777448 \mathrm{e}-01$ & \\
\hline 212 & Government & $6.598691 \mathrm{e}-02$ & $2.044891 \mathrm{e}-01$ & $7.469395 \mathrm{e}-01$ & \\
\hline 213 & Dentistry & $4.435792 \mathrm{e}-01$ & $3.849765 \mathrm{e}-01$ & $2.492726 \mathrm{e}-01$ & \\
\hline 214 & Microeconomics & $-3.951653 e-01$ & $3.245074 \mathrm{e}-01$ & $2.233687 \mathrm{e}-01$ & \\
\hline 215 & Sociology & $1.286958 \mathrm{e}-01$ & $4.117888 \mathrm{e}-01$ & $7.546491 \mathrm{e}-01$ & \\
\hline 216 & Emergency_medicine & $2.787570 \mathrm{e}-02$ & $8.573117 \mathrm{e}-02$ & $7.450765 \mathrm{e}-01$ & \\
\hline 217 & Pediatrics & $-1.003705 \mathrm{e}-01$ & $6.917502 \mathrm{e}-02$ & $1.468394 \mathrm{e}-01$ & \\
\hline 218 & Advertising & $3.186477 \mathrm{e}-01$ & $1.918371 \mathrm{e}-01$ & $9.675651 \mathrm{e}-02$ & \\
\hline 219 & Oceanography & $-6.560915 e-02$ & $2.819131 \mathrm{e}-01$ & $8.159799 \mathrm{e}-01$ & \\
\hline 220 & Developmental_psychology & $1.195245 \mathrm{e}-01$ & $1.034674 \mathrm{e}-01$ & $2.480562 \mathrm{e}-01$ & \\
\hline 221 & Internal_medicine & $8.220328 \mathrm{e}-02$ & $5.690524 \mathrm{e}-02$ & $1.486298 \mathrm{e}-01$ & \\
\hline 222 & Cardiology & $5.524896 \mathrm{e}-02$ & $8.955315 \mathrm{e}-02$ & $5.372962 \mathrm{e}-01$ & \\
\hline 223 & Environmental_engineering & $-8.506562 \mathrm{e}-02$ & $2.751861 \mathrm{e}-01$ & $7.572402 \mathrm{e}-01$ & \\
\hline 224 & General_surgery & $3.474743 \mathrm{e}-03$ & $1.107736 \mathrm{e}-01$ & $9.749771 \mathrm{e}-01$ & \\
\hline 225 & Environmental_science & $-2.655664 \mathrm{e}-01$ & $2.169341 \mathrm{e}-01$ & $2.209299 \mathrm{e}-01$ & \\
\hline 226 & Geophysics & $-6.860677 \mathrm{e}-02$ & $5.465873 e-01$ & $9.001171 \mathrm{e}-01$ & \\
\hline 227 & Immunology & $1.933034 \mathrm{e}-02$ & $7.518751 \mathrm{e}-02$ & $7.971137 \mathrm{e}-01$ & \\
\hline 228 & Clinical_psychology & $1.138828 \mathrm{e}-01$ & $1.061013 \mathrm{e}-01$ & $2.831598 \mathrm{e}-01$ & \\
\hline 229 & Toxicology & $8.617096 \mathrm{e}-01$ & $3.482656 \mathrm{e}-01$ & $1.337623 \mathrm{e}-02$ & $*$ \\
\hline 230 & Ecology & $7.529510 \mathrm{e}-02$ & $8.527012 \mathrm{e}-02$ & $3.772598 \mathrm{e}-01$ & \\
\hline 231 & Biology & $9.222282 \mathrm{e}-02$ & $1.596439 \mathrm{e}-01$ & $5.635019 \mathrm{e}-01$ & \\
\hline 232 & Epidemiology & $8.594034 \mathrm{e}-02$ & $8.753896 \mathrm{e}-02$ & $3.262664 \mathrm{e}-01$ & \\
\hline 233 & Physical_therapy & $2.620408 \mathrm{e}-01$ & $5.671623 \mathrm{e}-02$ & $3.909564 \mathrm{e}-06$ & $* * *$ \\
\hline 234 & Physiology & $-1.980659 \mathrm{e}-01$ & $2.178464 \mathrm{e}-01$ & $3.632795 \mathrm{e}-01$ & \\
\hline 235 & Statistics & $2.341798 \mathrm{e}-02$ & $6.805480 \mathrm{e}-01$ & $9.725509 \mathrm{e}-01$ & \\
\hline 236 & Psychopathology & $2.472524 \mathrm{e}-01$ & $2.503421 \mathrm{e}-01$ & $3.233580 \mathrm{e}-01$ & \\
\hline 237 & Animal_science & $3.515531 \mathrm{e}-02$ & $3.951403 \mathrm{e}-01$ & $9.291092 \mathrm{e}-01$ & \\
\hline 238 & Nursing & $2.257262 \mathrm{e}-01$ & $1.022985 \mathrm{e}-01$ & $2.738178 \mathrm{e}-02$ & * \\
\hline 239 & Seismology & $1.323521 \mathrm{e}-02$ & $4.299924 \mathrm{e}-01$ & $9.754459 \mathrm{e}-01$ & \\
\hline 240 & History & $1.381349 \mathrm{e}+00$ & $1.326173 e+00$ & $2.976343 \mathrm{e}-01$ & \\
\hline 241 & Psychiatry & $1.751021 \mathrm{e}-02$ & $7.313545 \mathrm{e}-02$ & $8.107864 \mathrm{e}-01$ & \\
\hline 242 & Zoology & $3.438307 \mathrm{e}-01$ & $1.880035 \mathrm{e}-01$ & $6.746886 \mathrm{e}-02$ & \\
\hline 243 & Psychology & $-4.618279 \mathrm{e}-03$ & $1.582133 \mathrm{e}-01$ & $9.767138 \mathrm{e}-01$ & \\
\hline 244 & Etiology & $3.132386 \mathrm{e}-01$ & $1.985362 \mathrm{e}-01$ & $1.146753 \mathrm{e}-01$ & \\
\hline 245 & Geomorphology & $3.468530 \mathrm{e}-01$ & $2.357002 \mathrm{e}-01$ & $1.411825 \mathrm{e}-01$ & \\
\hline 246 & Atmospheric_sciences & $3.069264 \mathrm{e}-01$ & $2.614331 \mathrm{e}-01$ & $2.404332 \mathrm{e}-01$ & \\
\hline 247 & Geography & $2.762497 \mathrm{e}-01$ & $2.213373 \mathrm{e}-01$ & $2.120431 \mathrm{e}-01$ & \\
\hline 248 & Demography & $6.952602 \mathrm{e}-02$ & $6.645468 \mathrm{e}-02$ & $2.955008 \mathrm{e}-01$ & \\
\hline 249 & Geology & $-6.022320 \mathrm{e}-01$ & $2.371066 \mathrm{e}-01$ & $1.111151 \mathrm{e}-02$ & $*$ \\
\hline 250 & Medicine & $9.558576 \mathrm{e}-02$ & $1.369642 \mathrm{e}-01$ & $4.852724 \mathrm{e}-01$ & \\
\hline 251 & Family_medicine & $-1.230967 \mathrm{e}-01$ & $6.874645 \mathrm{e}-02$ & $7.340696 \mathrm{e}-02$ & \\
\hline 252 & Evolutionary_biology & $-1.388986 \mathrm{e}-01$ & $1.670676 \mathrm{e}-01$ & $4.057836 \mathrm{e}-01$ & \\
\hline 253 & Astrobiology & $6.528570 \mathrm{e}-01$ & $3.667338 \mathrm{e}-01$ & $7.509240 \mathrm{e}-02$ & \\
\hline 254 & Astronomy & $1.452574 \mathrm{e}-01$ & $2.820646 \mathrm{e}-01$ & $6.065856 \mathrm{e}-01$ & \\
\hline 255 & Astrophysics & $-4.785610 \mathrm{e}-02$ & $4.135766 \mathrm{e}-01$ & $9.078839 \mathrm{e}-01$ & \\
\hline 256 & Geochemistry & $1.369776 \mathrm{e}-01$ & $4.036109 \mathrm{e}-01$ & $7.343346 \mathrm{e}-01$ & \\
\hline 257 & Business & $3.041133 \mathrm{e}-02$ & $2.331766 \mathrm{e}-01$ & $8.962368 \mathrm{e}-01$ & \\
\hline 258 & Finance & $1.940427 \mathrm{e}-02$ & $2.396875 \mathrm{e}-01$ & $9.354791 \mathrm{e}-01$ & \\
\hline 259 & Management_science & $-4.253369 \mathrm{e}-14$ & $5.514719 \mathrm{e}-14$ & $4.405723 \mathrm{e}-01$ & \\
\hline 260 & Social_psychology & $4.484812 \mathrm{e}-02$ & $8.051094 \mathrm{e}-02$ & $5.775172 \mathrm{e}-01$ & \\
\hline 261 & Political_science & $-2.722226 \mathrm{e}-01$ & $3.667877 \mathrm{e}-01$ & $4.580057 \mathrm{e}-01$ & \\
\hline 262 & Archaeology & $4.512896 \mathrm{e}-01$ & $3.544401 \mathrm{e}-01$ & $2.029775 \mathrm{e}-01$ & \\
\hline 263 & Economics & $1.880270 \mathrm{e}-01$ & $1.833695 \mathrm{e}-01$ & $3.052140 \mathrm{e}-01$ & \\
\hline 264 & Paleontology & $1.245241 \mathrm{e}-02$ & $2.222471 \mathrm{e}-01$ & $9.553200 \mathrm{e}-01$ & \\
\hline 265 & Criminology & $4.386701 \mathrm{e}-01$ & $5.081198 \mathrm{e}-01$ & $3.879942 \mathrm{e}-01$ & \\
\hline
\end{tabular}

Table 8: Regression coefficients for predicting sentence-level certainty in news with aspect-level certainty in abstracts (RQ3). 


\begin{tabular}{|c|c|c|c|c|c|}
\hline & IV & coeff & SE & $\mathrm{p}$ value & \\
\hline 0 & (Intercept) & $4.769169 \mathrm{e}+00$ & 0.090361 & $0.000000 \mathrm{e}+00$ & $* * *$ \\
\hline 1 & finding_len & $-8.585722 \mathrm{e}-03$ & 0.000329 & $2.223725 \mathrm{e}-149$ & $* * *$ \\
\hline 2 & flesch_reading_ease & $-1.233028 \mathrm{e}-03$ & 0.000127 & $3.486418 \mathrm{e}-22$ & $* * *$ \\
\hline 3 & first_author_rank & $6.810203 e-06$ & 0.000002 & $2.893930 \mathrm{e}-05$ & $* * *$ \\
\hline 4 & affi_rank & $-1.955582 \mathrm{e}-07$ & 0.000002 & $9.088450 \mathrm{e}-01$ & \\
\hline 5 & affi_cateinternational & $6.176708 \mathrm{e}-03$ & 0.006035 & $3.061173 \mathrm{e}-01$ & \\
\hline 6 & affi_cateunknown & 7.980791e-02 & 0.066196 & $2.279631 \mathrm{e}-01$ & \\
\hline 7 & journal_impact_cat>30 & $1.115552 \mathrm{e}-02$ & 0.011485 & $3.314135 \mathrm{e}-01$ & \\
\hline 8 & journal_impact_cat0 & $3.484480 \mathrm{e}-02$ & 0.038634 & $3.671009 \mathrm{e}-01$ & \\
\hline 9 & journal_impact_cat1 & $2.338580 \mathrm{e}-02$ & 0.016713 & $1.617297 \mathrm{e}-01$ & \\
\hline 10 & journal_impact_cat10 & $3.099042 \mathrm{e}-02$ & 0.021125 & $1.423854 \mathrm{e}-01$ & \\
\hline 11 & journal_impact_cat11-20 & $-1.325421 \mathrm{e}-02$ & 0.011667 & $2.559562 \mathrm{e}-01$ & \\
\hline 12 & journal_impact_cat2 & $-2.835769 e-03$ & 0.013643 & $8.353414 \mathrm{e}-01$ & \\
\hline 13 & journal_impact_cat21-30 & $1.157902 \mathrm{e}-02$ & 0.016218 & $4.752635 \mathrm{e}-01$ & \\
\hline 14 & journal_impact_cat3 & $-2.153386 e-02$ & 0.013002 & $9.768855 \mathrm{e}-02$ & \\
\hline 15 & journal_impact_cat4 & $-1.383440 \mathrm{e}-02$ & 0.012566 & $2.709385 \mathrm{e}-01$ & \\
\hline 16 & journal_impact_cat5 & $7.237343 \mathrm{e}-03$ & 0.014383 & $6.148402 \mathrm{e}-01$ & \\
\hline 17 & journal_impact_cat6 & $-1.279505 e-03$ & 0.017806 & $9.427163 \mathrm{e}-01$ & \\
\hline 18 & journal_impact_cat7 & $-4.565138 \mathrm{e}-03$ & 0.016952 & $7.876998 \mathrm{e}-01$ & \\
\hline 19 & journal_impact_cat8 & $-1.158010 \mathrm{e}-02$ & 0.019421 & $5.509985 \mathrm{e}-01$ & \\
\hline 20 & num_authors_cat>30 & $-6.860334 \mathrm{e}-02$ & 0.020412 & $7.774055 \mathrm{e}-04$ & $* * *$ \\
\hline 21 & num_authors_cat1 & $-5.658062 \mathrm{e}-02$ & 0.020951 & $6.922228 \mathrm{e}-03$ & $* *$ \\
\hline 22 & num_authors_cat10 & $-4.269129 \mathrm{e}-02$ & 0.018650 & $2.207752 \mathrm{e}-02$ & $*$ \\
\hline 23 & num_authors_cat11-20 & $-3.377127 \mathrm{e}-02$ & 0.014301 & $1.820837 \mathrm{e}-02$ & $*$ \\
\hline 24 & num_authors_cat2 & $-7.501011 \mathrm{e}-02$ & 0.015725 & $1.845783 \mathrm{e}-06$ & $* * *$ \\
\hline 25 & num_authors_cat21-30 & $-2.494395 e-02$ & 0.018716 & $1.826133 \mathrm{e}-01$ & \\
\hline 26 & num_authors_cat3 & $-4.136119 \mathrm{e}-02$ & 0.015437 & $7.376600 \mathrm{e}-03$ & $* *$ \\
\hline 27 & num_authors_cat4 & $-4.854558 \mathrm{e}-02$ & 0.015367 & $1.582984 \mathrm{e}-03$ & $* *$ \\
\hline 28 & num_authors_cat5 & $-5.129608 \mathrm{e}-02$ & 0.015485 & $9.244715 \mathrm{e}-04$ & $* * *$ \\
\hline 29 & num_authors_cat6 & $-3.495075 e-02$ & 0.015828 & $2.723927 \mathrm{e}-02$ & $*$ \\
\hline 30 & num_authors_cat7 & $-4.805026 \mathrm{e}-02$ & 0.016237 & $3.084075 \mathrm{e}-03$ & $* *$ \\
\hline 31 & num_authors_cat8 & $-2.308659 \mathrm{e}-02$ & 0.016820 & $1.698922 \mathrm{e}-01$ & \\
\hline 32 & outlet_re12 News KBMT & $1.418467 \mathrm{e}-01$ & 0.167373 & $3.967259 \mathrm{e}-01$ & \\
\hline 33 & outlet_re2 Minute Medicine & $-4.312124 \mathrm{e}-02$ & 0.087548 & $6.223354 \mathrm{e}-01$ & \\
\hline 34 & outlet_re7th Space Family Portal & $-4.599097 \mathrm{e}-02$ & 0.091995 & $6.171269 \mathrm{e}-01$ & \\
\hline 35 & outlet_reABC 7 WKBW Buffalo & $-8.998381 \mathrm{e}-02$ & 0.256673 & $7.259058 \mathrm{e}-01$ & \\
\hline 36 & outlet_reABC Action News WFTS Tampa Bay & $-2.982455 e-01$ & 0.244460 & $2.224629 \mathrm{e}-01$ & \\
\hline 37 & outlet_reABC News & $-6.698653 e-02$ & 0.093044 & $4.715628 \mathrm{e}-01$ & \\
\hline 38 & outlet_reABC News 15 Arizona & $1.766278 \mathrm{e}-01$ & 0.270452 & $5.137049 \mathrm{e}-01$ & \\
\hline 39 & outlet_reABC News WMUR 9 & $-3.542851 \mathrm{e}-01$ & 0.129487 & $6.219294 \mathrm{e}-03$ & $* *$ \\
\hline 40 & outlet_reAction News Now & $-5.668414 \mathrm{e}-02$ & 0.102343 & $5.796746 \mathrm{e}-01$ & \\
\hline 41 & outlet_reAJMC & $-1.393673 e-01$ & 0.095787 & $1.456802 \mathrm{e}-01$ & \\
\hline 42 & outlet_reAlaska Despatch News & $9.177018 \mathrm{e}-02$ & 0.123984 & $4.591945 \mathrm{e}-01$ & \\
\hline 43 & outlet_reAlternet & $-1.114754 \mathrm{e}-01$ & 0.094173 & $2.365219 \mathrm{e}-01$ & \\
\hline 44 & outlet_reAmerican Council on Science and Health & $-9.416221 \mathrm{e}-02$ & 0.096104 & $3.271910 \mathrm{e}-01$ & \\
\hline 45 & outlet_reAmerican Physical Society - Physics & $-2.596486 \mathrm{e}-01$ & 0.102138 & $1.101966 \mathrm{e}-02$ & $*$ \\
\hline 46 & outlet_reAOL & $-7.593740 \mathrm{e}-02$ & 0.429942 & $8.598053 \mathrm{e}-01$ & \\
\hline 47 & outlet_reArizona Public Radio & $-1.542826 \mathrm{e}-01$ & 0.234993 & $5.114788 \mathrm{e}-01$ & \\
\hline 48 & outlet_reArstechnica & $-2.321924 \mathrm{e}-01$ & 0.112495 & $3.901870 \mathrm{e}-02$ & $*$ \\
\hline 49 & outlet_reAstrobiology Magazine & $-2.117611 \mathrm{e}-01$ & 0.103951 & $4.164215 \mathrm{e}-02$ & $*$ \\
\hline 50 & outlet_reazfamily.com & $-2.734613 e-01$ & 0.097476 & $5.026437 \mathrm{e}-03$ & $* *$ \\
\hline 51 & outlet_reBecker's Hospital Review & $1.236054 \mathrm{e}-02$ & 0.105464 & $9.067007 \mathrm{e}-01$ & \\
\hline 52 & outlet_reBecker's Spine Review & $1.118130 \mathrm{e}-02$ & 0.178829 & $9.501449 \mathrm{e}-01$ & \\
\hline 53 & outlet_reBenzinga & $-8.756726 \mathrm{e}-02$ & 0.089176 & $3.261230 \mathrm{e}-01$ & \\
\hline 54 & outlet_reBillings Gazette & $-3.004539 \mathrm{e}-02$ & 0.166643 & $8.569189 \mathrm{e}-01$ & \\
\hline 55 & outlet_reBio-Medicine.org & $-1.291493 e-01$ & 0.123512 & $2.957305 \mathrm{e}-01$ & \\
\hline 56 & outlet_reBiospace & $-8.649325 \mathrm{e}-02$ & 0.087187 & $3.211818 \mathrm{e}-01$ & \\
\hline 57 & outlet_reBioTech Gate & $-2.053865 e-01$ & 0.118983 & $8.431746 \mathrm{e}-02$ & \\
\hline 58 & outlet_reBoise State Public Radio & $4.968309 \mathrm{e}-02$ & 0.308591 & $8.720939 \mathrm{e}-01$ & \\
\hline 59 & outlet_reBreitbart News Network & $-7.084124 \mathrm{e}-02$ & 0.217911 & $7.451118 \mathrm{e}-01$ & \\
\hline 60 & outlet_reBrookings & $-6.427207 e-03$ & 0.102487 & $9.499958 \mathrm{e}-01$ & \\
\hline 61 & outlet_reBusiness Insider & $-1.177954 \mathrm{e}-01$ & 0.098250 & $2.305574 \mathrm{e}-01$ & \\
\hline 62 & outlet_reBusiness Wire & $3.400983 \mathrm{e}-01$ & 0.256321 & $1.845636 \mathrm{e}-01$ & \\
\hline 63 & outlet_reBustle & $-1.959370 \mathrm{e}-01$ & 0.096802 & $4.296269 \mathrm{e}-02$ & $*$ \\
\hline 64 & outlet_reBuzzfeed & $-1.476767 \mathrm{e}-01$ & 0.211114 & $4.842350 \mathrm{e}-01$ & \\
\hline 65 & outlet_reCarbon Brief & $-2.223582 \mathrm{e}-01$ & 0.094952 & $1.919420 \mathrm{e}-02$ & $*$ \\
\hline 66 & outlet_reCardiovascular Business & $-3.031564 \mathrm{e}-02$ & 0.144638 & $8.339826 \mathrm{e}-01$ & \\
\hline
\end{tabular}


outlet_reCBS News

outlet_reCentre for Disease Research and Policy

outlet_reChristian Science Monitor

outlet_reClinical Advisor

outlet_reCNBC

outlet_reCNET

outlet_reCNN News

outlet_reCommon Dreams

outlet_reCornell Chronicle

outlet_reCounsel \& Heal

outlet_reDaily Kos

outlet_reDayton Daily News

outlet_reDiscover Magazine

outlet_reDoctors Lounge

outlet_reDrug Discovery and Development

outlet_reDrugs.com

outlet_reEarthSky

outlet_reECN

outlet_reElite Daily

outlet_reEmaxhealth.com

outlet_reEnvironmental News Network

outlet_reEquities.com

outlet_reEurekAlert!

outlet_reEveryday Health

outlet_reFast Company

outlet_reFight Aging!

outlet_reFiveThirtyEight

outlet_reFOX News

outlet_reFuturity

outlet_reGEN

outlet_reGeorgia Public Radio

outlet_reGoogle News

outlet_reGovernment Executive

outlet_reGreen Car Congress

outlet_reGuardian Liberty Voice

outlet_reHarvard Business Review

outlet_reHeadlines \& Global News

outlet_reHealth

outlet_reHealth Canal

outlet_reHealth Day

outlet_reHealth Imaging

outlet_reHealth Medicinet

outlet_reHealthline

outlet_rehellogiggles.com

outlet_reHerald Sun

outlet_reHerald Tribune

outlet_reHomeland Security News Wire

outlet_reHouston Chronicle

outlet_reHowStuffWorks

outlet_reHuffington Post

outlet_reIdaho Statements

outlet_reInc.

outlet_reInfection Control Today

outlet_reInside Science

outlet_reInsurance News Net

outlet_reInternational Business Times

outlet_reInverse

outlet_reIowa Public Radio

outlet_reJournalist's Resource

outlet_reKansas City Star

outlet_reKansas City University Radio

outlet_reKansas Public Radio

outlet_reKCENG12

outlet_reKERA News

outlet_reKiiiTV 3

outlet_reKing 5

outlet_reKOSU

outlet_reKPBS

outlet_reKPCC : Southern California Public Radio

outlet_reKRWG TV/FM
$-1.057902 \mathrm{e}-01$

$1.289307 \mathrm{e}-01$

$-2.282957 \mathrm{e}-02$

$-7.162084 \mathrm{e}-02$

$5.914617 \mathrm{e}-02$

$4.275404 \mathrm{e}-02$

$-2.990173 \mathrm{e}-01$

$-1.647337 \mathrm{e}-01$

$-8.121157 \mathrm{e}-02$

$-2.143805 \mathrm{e}-02$

$2.300905 \mathrm{e}-02$

$8.135284 \mathrm{e}-02$

$1.773501 \mathrm{e}-02$

$-2.875162 \mathrm{e}-02$

$-1.270650 \mathrm{e}-01$

$4.794902 \mathrm{e}-03$

$-1.043942 \mathrm{e}-01$

$-5.907096 \mathrm{e}-02$

$-6.227301 \mathrm{e}-02$

$1.496814 \mathrm{e}-02$

$-1.580648 \mathrm{e}-01$

$-4.579956 \mathrm{e}-02$

$-6.943812 \mathrm{e}-02$

$-1.493808 \mathrm{e}-01$

$-1.643770 \mathrm{e}-01$

$-8.325870 \mathrm{e}-02$

$-1.347739 \mathrm{e}-01$

$-2.000903 \mathrm{e}-01$

$-9.837692 \mathrm{e}-02$

$-1.224438 \mathrm{e}-01$

$-1.360975 \mathrm{e}-01$

$-1.390805 \mathrm{e}-01$

$-1.144597 \mathrm{e}-01$

$5.236885 \mathrm{e}-02$

$-4.884608 \mathrm{e}-02$

$-1.502870 \mathrm{e}-01$

$6.714558 \mathrm{e}-03$

$-4.375126 \mathrm{e}-02$

$-7.192425 \mathrm{e}-02$

$-1.811595 \mathrm{e}-01$

$-1.013049 \mathrm{e}-01$

$-1.197016 \mathrm{e}-01$

5.041231e-04

$-1.521049 \mathrm{e}-01$

$1.840454 \mathrm{e}-02$

$-7.896818 \mathrm{e}-02$

$-2.311931 \mathrm{e}-01$

$-1.264263 \mathrm{e}-01$

$-1.085633 \mathrm{e}-01$

$-1.753507 \mathrm{e}-01$

$-1.367420 \mathrm{e}-01$

$-2.885615 \mathrm{e}-02$

$-4.932433 \mathrm{e}-02$

$-1.394995 \mathrm{e}-01$

$-1.118142 \mathrm{e}-01$

$-8.918184 \mathrm{e}-02$

$-1.340195 \mathrm{e}-01$

$8.488846 \mathrm{e}-02$

$-1.261923 \mathrm{e}-01$

$1.827350 \mathrm{e}-01$

$1.907765 \mathrm{e}-02$

$-3.241566 \mathrm{e}-02$

$-2.303815 \mathrm{e}-01$

3.279992e-02

$-3.457711 \mathrm{e}-01$

$-7.172016 \mathrm{e}-02$

$-2.588662 \mathrm{e}-01$

$-7.959956 \mathrm{e}-02$

$-6.482439 \mathrm{e}-02$

$-3.408372 \mathrm{e}-01$
0.091276

0.150621

0.090961

0.094883

0.270347

0.189956

0.172478

0.099371

0.117048

0.092798

0.244820

0.127584

0.178653

0.089025

0.134709

0.085832

0.088849

0.100715

0.091942

0.093292

0.091333

0.106006

0.085482

0.088384

0.101322

0.089406

0.119805

0.159959

0.081818

0.088232

0.139693

0.092665

0.112339

0.105622

0.087284

0.109402

0.084716

0.110842

0.081390

0.154427

0.140246

0.081891

0.118510

0.128123

0.157959

0.113124

0.095445

0.111696

0.091457

0.096591

0.205719

0.092435

0.096901

0.115661

0.116388

0.085132

0.082395

0.270454

0.096084

0.431490

0.190358

0.181981

0.308981

0.186044

0.138292

0.140876

0.336716

0.135971

0.127915

0.186725 2.464531e-01

$3.920055 \mathrm{e}-01$

$8.018283 \mathrm{e}-01$

$4.503524 \mathrm{e}-01$

$8.268232 \mathrm{e}-01$

$8.219228 \mathrm{e}-01$

$8.298300 \mathrm{e}-02$

$9.736787 \mathrm{e}-02$

4.877897e-01

$8.173015 \mathrm{e}-01$

$9.251225 \mathrm{e}-01$

$5.237081 \mathrm{e}-01$

9.209237e-01

$7.467259 \mathrm{e}-01$

$3.455532 \mathrm{e}-01$

$9.554506 \mathrm{e}-01$

$2.400150 \mathrm{e}-01$

$5.575317 \mathrm{e}-01$

$4.982124 \mathrm{e}-01$

$8.725321 \mathrm{e}-01$

$8.351947 \mathrm{e}-02$

$6.657094 \mathrm{e}-01$

$4.166176 \mathrm{e}-01$

9.100728e-02

$1.047374 \mathrm{e}-01$

$3.517325 \mathrm{e}-01$

$2.606163 \mathrm{e}-01$

2.109811e-01

2.292193e-01

$1.652196 \mathrm{e}-01$

$3.299296 \mathrm{e}-01$

$1.333882 \mathrm{e}-01$

$3.082658 \mathrm{e}-01$

$6.200273 \mathrm{e}-01$

$5.757369 \mathrm{e}-01$

$1.695334 \mathrm{e}-01$

$9.368267 \mathrm{e}-01$

$6.930523 \mathrm{e}-01$

$3.768590 \mathrm{e}-01$

$2.407556 \mathrm{e}-01$

$4.700892 \mathrm{e}-01$

$1.438250 \mathrm{e}-01$

$9.966059 \mathrm{e}-01$

$2.351606 \mathrm{e}-01$

9.072447e-01

$4.851392 \mathrm{e}-01$

$1.542642 \mathrm{e}-02$

$2.576873 \mathrm{e}-01$

$2.352169 \mathrm{e}-01$

$6.946858 \mathrm{e}-02$

$5.062429 \mathrm{e}-01$

$7.549076 \mathrm{e}-01$

$6.107402 \mathrm{e}-01$

$2.277802 \mathrm{e}-01$

$3.367068 \mathrm{e}-01$

$2.948382 \mathrm{e}-01$

$1.038382 \mathrm{e}-01$

$7.536174 \mathrm{e}-01$

$1.890678 \mathrm{e}-01$

$6.719338 \mathrm{e}-01$

$9.201699 \mathrm{e}-01$

$8.586239 \mathrm{e}-01$

$4.559008 \mathrm{e}-01$

8.600572e-01

$1.241090 \mathrm{e}-02$

$6.106830 \mathrm{e}-01$

4.420162e-01

$5.582689 \mathrm{e}-01$

6.123117e-01

$6.795268 \mathrm{e}-02$ 


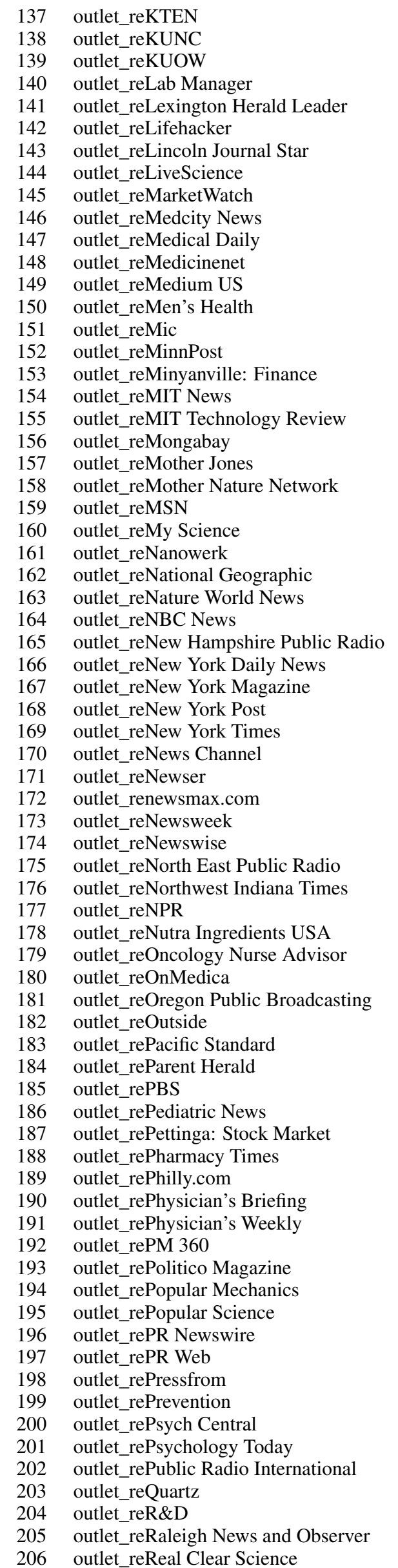

$-9.834243 e-02$

$-1.704335 \mathrm{e}-01$

$1.493518 \mathrm{e}-02$

$-7.857505 \mathrm{e}-02$

$-3.583428 \mathrm{e}-01$

$-8.568183 \mathrm{e}-02$

$-1.609512 \mathrm{e}-02$

$-1.064932 \mathrm{e}-01$

$-9.276407 \mathrm{e}-03$

$-8.197926 \mathrm{e}-02$

$-1.106447 \mathrm{e}-01$

$-9.953593 e-02$

$-1.122500 \mathrm{e}-01$

$1.242141 \mathrm{e}-01$

$-2.714787 \mathrm{e}-01$

$-1.199738 \mathrm{e}-01$

$2.548422 \mathrm{e}-02$

$-9.046612 \mathrm{e}-03$

$-1.470581 \mathrm{e}-01$

$-1.568619 \mathrm{e}-01$

$-3.759236 \mathrm{e}-01$

$-9.544112 \mathrm{e}-02$

$-1.301312 \mathrm{e}-01$

$-3.489837 \mathrm{e}-02$

$2.003345 \mathrm{e}-02$

$-4.082564 \mathrm{e}-02$

$-1.211760 \mathrm{e}-01$

$1.114485 \mathrm{e}-01$

$-1.321984 \mathrm{e}-01$

$-5.149885 \mathrm{e}-02$

$-1.704247 \mathrm{e}-03$

$1.488574 \mathrm{e}-01$

$-5.607231 \mathrm{e}-02$

8.213177e-02

$-5.894877 \mathrm{e}-02$

$-1.081776 \mathrm{e}-01$

$-9.483019 \mathrm{e}-02$

$-9.817610 \mathrm{e}-02$

$-1.937664 \mathrm{e}-01$

4.126759e-03

$-1.175292 \mathrm{e}-01$

6.983471e-02

$-3.679552 \mathrm{e}-02$

$4.962610 \mathrm{e}-02$

$-4.380958 \mathrm{e}-01$

$-2.098173 \mathrm{e}-01$

$-1.278349 \mathrm{e}-01$

$-7.227167 \mathrm{e}-02$

$-1.332501 \mathrm{e}-01$

$-8.104710 \mathrm{e}-02$

$-1.557469 \mathrm{e}-01$

$-1.179944 \mathrm{e}-01$

$-1.826453 \mathrm{e}-01$

$-1.166461 \mathrm{e}-02$

$-9.399661 \mathrm{e}-02$

$-1.123080 \mathrm{e}-01$

$-5.817698 \mathrm{e}-01$

$-1.249849 \mathrm{e}-01$

$-1.515766 \mathrm{e}+00$

$-6.320346 \mathrm{e}-02$

$-1.307000 \mathrm{e}-01$

$-9.822203 \mathrm{e}-02$

$-1.145023 \mathrm{e}-01$

$-1.623597 \mathrm{e}-01$

$2.012517 \mathrm{e}-02$

$-7.554060 \mathrm{e}-02$

$-1.553909 \mathrm{e}-01$

6.907842e-02

$-1.484769 \mathrm{e}-01$

$-1.057347 \mathrm{e}-01$
0.143354

0.122598

0.217831

0.090292

0.211151

0.143355

0.147410

0.084400

0.103723

0.108809

0.081733

0.087098

0.083424

0.159841

0.110274

0.096098

0.190078

0.095727

0.200698

0.086476

0.186148

0.086099

0.091907

0.117097

0.084120

0.107597

0.082059

0.154169

0.257018

0.103295

0.143413

0.429672

0.095775

0.144154

0.091765

0.101941

0.138609

0.081690

0.139442

0.138152

0.157668

0.099478

0.085922

0.164375

0.199689

0.105426

0.089554

0.089470

0.086110

0.087781

0.428893

0.118284

0.098743

0.225665

0.142341

0.144289

0.245738

0.274259

0.428817

0.114111

0.109985

0.091395

0.094920

0.085102

0.094449

0.113615

0.097070

0.244635

0.176008

0.090167 4.927093e-01

$1.644783 \mathrm{e}-01$

$9.453376 \mathrm{e}-01$

$3.841746 \mathrm{e}-01$

$8.968359 \mathrm{e}-02$

$5.500498 \mathrm{e}-01$

9.130554e-01

2.070392e-01

9.287367e-01

4.511961e-01

$1.758279 \mathrm{e}-01$

$2.531216 \mathrm{e}-01$

$1.784587 \mathrm{e}-01$

$4.370972 \mathrm{e}-01$

$1.382411 \mathrm{e}-02$

$2.118700 \mathrm{e}-01$

$8.933459 \mathrm{e}-01$

9.247091e-01

4.637228e-01

$6.969115 \mathrm{e}-02$

$4.343985 \mathrm{e}-02$

$2.676454 \mathrm{e}-01$

$1.568085 \mathrm{e}-01$

$7.656812 \mathrm{e}-01$

$8.117638 \mathrm{e}-01$

7.043701e-01

$1.397624 \mathrm{e}-01$

$4.697460 \mathrm{e}-01$

$6.070054 \mathrm{e}-01$

6.180894e-01

9.905186e-01

$7.290098 \mathrm{e}-01$

$5.582393 \mathrm{e}-01$

$5.688500 \mathrm{e}-01$

$5.206239 \mathrm{e}-01$

$2.886139 \mathrm{e}-01$

4.938786e-01

2.294406e-01

$1.646596 \mathrm{e}-01$

9.761699e-01

$4.560187 \mathrm{e}-01$

4.826746e-01

$6.684741 \mathrm{e}-01$

$7.627233 \mathrm{e}-01$

$2.824662 \mathrm{e}-02$

4.657494e-02

$1.534532 \mathrm{e}-01$

4.192243e-01

$1.217614 \mathrm{e}-01$

$3.558585 \mathrm{e}-01$

$7.165035 \mathrm{e}-01$

$3.185005 \mathrm{e}-01$

$6.435964 \mathrm{e}-02$

9.587759e-01

$5.090236 \mathrm{e}-01$

4.363622e-01

$1.791423 \mathrm{e}-02$

$6.485940 \mathrm{e}-01$

$4.083987 \mathrm{e}-04$

$5.796654 \mathrm{e}-01$

$2.347001 \mathrm{e}-01$

$2.825115 \mathrm{e}-01$

$2.277022 \mathrm{e}-01$

5.641802e-02

8.312652e-01

$5.061276 \mathrm{e}-01$

$1.094238 \mathrm{e}-01$

7.776582e-01

$3.989065 \mathrm{e}-01$

$2.409384 \mathrm{e}-01$ 
outlet_reReason outlet_reredOrbit outlet_reRelief Web outlet_reRenal \& Urology News outlet_reRocket News outlet_reRunner's World outlet_reSalon outlet_reSci-News outlet_reScience 2.0 outlet_reScience Alert outlet_reScience Daily outlet_reScience World Report outlet_reScience/AAAS outlet_reScientific American outlet_reSeed Daily outlet_reSeedQuest outlet_reSFGate outlet_reShe Knows outlet_reSign of the Times outlet_reSky Nightly outlet_reSlate Magazine outlet_reSmithsonian Magazine outlet_reSpace Daily outlet_reSpace.com outlet_reSPIE Newsroom outlet_reSpringfield News Sun outlet_reSt. Louis Post-Dispatch outlet_reStar Tribune outlet_reStatesman.com outlet_reTCTMD outlet_reTech Times outlet_reTech Xplore outlet_reTechnology Networks outlet_reTechnology.org outlet_reThe Advocate outlet_reThe ASCO Post outlet_reThe Atlanta Journal Constitution outlet_reThe Atlantic outlet_reThe Body outlet_reThe Columbian outlet_reThe Daily Beast outlet_reThe Daily Caller outlet_reThe Daily Meal outlet_reThe Denver Post outlet_reThe Deseret News outlet_reThe Ecologist outlet_reThe Epoch Times outlet_reThe Hill outlet_reThe Inquisitr outlet_reThe Modesto Bee outlet_reThe New York Observer outlet_reThe Raw Story outlet_reThe Sacramento Bee outlet_reThe San Diego Union-Tribune outlet_reThe Seattle Times outlet_reThe University Herald outlet_reThe University of New Orleans Public ... outlet_reThe Verge outlet_reThe Week outlet_reTIME Magazine outlet_reTODAY outlet_reUBM Medica outlet_reUPI.com outlet_reUS News Health outlet_reUSA Today outlet_reUSNews.com outlet_reValue Walk outlet_reVice outlet_reVoice of America outlet_reVox.com
$-7.172918 \mathrm{e}-02$

$-1.194361 \mathrm{e}-01$

$-7.076507 \mathrm{e}-02$

$-2.514014 \mathrm{e}-01$

$1.615605 \mathrm{e}-01$

$-1.504648 \mathrm{e}-01$

$-1.265607 \mathrm{e}-01$

$-9.216745 \mathrm{e}-02$

$-8.690223 \mathrm{e}-02$

$-1.393268 \mathrm{e}-01$

$-3.342673 \mathrm{e}-02$

$-1.711869 \mathrm{e}-01$

$-2.003872 \mathrm{e}-01$

$-1.608189 \mathrm{e}-01$

$-1.718862 \mathrm{e}-01$

$-1.687705 \mathrm{e}-01$

$-7.066610 \mathrm{e}-02$

$-4.378007 \mathrm{e}-02$

$-1.401738 \mathrm{e}-01$

$-1.358002 \mathrm{e}-01$

$2.381837 \mathrm{e}-02$

$-9.156631 \mathrm{e}-02$

$-1.533121 \mathrm{e}-01$

$-2.754762 \mathrm{e}-01$

$-9.738364 \mathrm{e}-02$

$6.652785 \mathrm{e}-02$

$-5.640932 \mathrm{e}-01$

$1.211137 \mathrm{e}-02$

$-2.288543 \mathrm{e}-01$

2.104453e-01

$-7.274466 \mathrm{e}-02$

$-2.132806 \mathrm{e}-01$

$-3.707963 e-02$

$-9.818053 \mathrm{e}-02$

$8.886938 \mathrm{e}-02$

$3.041240 \mathrm{e}-03$

$-1.607467 \mathrm{e}-01$

$-6.557165 \mathrm{e}-02$

$-1.484161 \mathrm{e}-01$

$-2.789612 \mathrm{e}-01$

$-2.640513 \mathrm{e}-01$

$-1.083757 \mathrm{e}-01$

$6.904495 \mathrm{e}-02$

$-1.554902 \mathrm{e}-01$

$8.105423 \mathrm{e}-03$

$-2.993036 \mathrm{e}-02$

$-9.873554 \mathrm{e}-02$

$-8.941341 \mathrm{e}-02$

$-2.177188 \mathrm{e}-01$

$-6.710310 \mathrm{e}-01$

$-3.049783 \mathrm{e}-01$

$-1.132624 \mathrm{e}-01$

$-1.678217 \mathrm{e}-01$

$-8.874699 \mathrm{e}-02$

$-1.884026 \mathrm{e}-01$

$-1.324061 \mathrm{e}-01$

$-1.840991 \mathrm{e}-01$

$-1.801690 \mathrm{e}-01$

$-2.083448 \mathrm{e}-01$

$-1.551461 \mathrm{e}-01$

$-2.206053 \mathrm{e}-01$

$-3.766528 \mathrm{e}-02$

$-1.563671 \mathrm{e}-01$

$-1.110524 \mathrm{e}-01$

$-1.745214 \mathrm{e}-01$

$-1.272654 \mathrm{e}-01$

$-1.194338 \mathrm{e}-01$

$-1.157334 \mathrm{e}-01$

$1.188659 \mathrm{e}-02$

$-1.521713 \mathrm{e}-01$
0.100165

0.085498

0.139495

0.113343

0.308731

0.092001

0.107117

0.084148

0.084530

0.083442

0.081747

0.082404

0.093369

0.089883

0.094259

0.108636

0.144596

0.092903

0.083003

0.091466

0.131770

0.105474

0.088813

0.190987

0.144411

0.244409

0.175363

0.103912

0.150263

0.144007

0.107746

0.114480

0.082963

0.081028

0.179171

0.087142

0.135414

0.096151

0.120679

0.094597

0.099946

0.116698

0.131389

0.186026

0.120665

0.123181

0.083186

0.092783

0.087619

0.212488

0.205597

0.086479

0.170188

0.104396

0.096942

0.094654

0.140553

0.104278

0.112975

0.124589

0.181992

0.086759

0.083897

0.167373

0.211178

0.100129

0.126310

0.111034

0.130311

0.108516

4.739262e-01

$1.624348 \mathrm{e}-01$

$6.119504 \mathrm{e}-01$

$2.655434 \mathrm{e}-02$

$6.007641 \mathrm{e}-01$

$1.019534 \mathrm{e}-01$

$2.374001 \mathrm{e}-01$

$2.733879 \mathrm{e}-01$

3.039221e-01

9.497571e-02 


\begin{tabular}{|c|c|}
\hline 277 & outlet_reWABE \\
\hline 278 & outlet_reWashington Post \\
\hline 279 & outlet_reWBUR \\
\hline 280 & outlet_reWCBE \\
\hline 281 & outlet_reWebMD News \\
\hline 282 & outlet_reWinona Daily News \\
\hline 283 & outlet_reWired.com \\
\hline 284 & outlet_reWitchita's Public Radio \\
\hline 285 & outlet_reWJCT \\
\hline 286 & outlet_reWLRN \\
\hline 287 & outlet_reWomen's Health \\
\hline 288 & outlet_reWPTV 5 West Palm Beach \\
\hline 289 & outlet_reWTOP \\
\hline 290 & outlet_reWUNC \\
\hline 291 & outlet_reWUWM \\
\hline 292 & outlet_reWVXU \\
\hline 293 & outlet_reWyoming Public Radio \\
\hline 294 & outlet_reWYPR \\
\hline 295 & outlet_reYahoo! \\
\hline 296 & outlet_reYahoo! Finance USA \\
\hline 297 & outlet_reYahoo! News \\
\hline 298 & Law \\
\hline 299 & Condensed_matter_physics \\
\hline 300 & Biomedical_engineering \\
\hline 301 & Atomic_physics \\
\hline 302 & Development_economics \\
\hline 303 & Optics \\
\hline 304 & Public_policy \\
\hline 305 & Lung_cancer \\
\hline 306 & Electronic_engineering \\
\hline 307 & Artificial_intelligence \\
\hline 308 & Machine_learning \\
\hline 309 & Gender_studies \\
\hline 310 & Biophysics \\
\hline 311 & Analytical_chemistry \\
\hline 312 & Computer_science \\
\hline 313 & Radiology \\
\hline 314 & Urology \\
\hline 315 & Architecture \\
\hline 316 & Quantum_mechanics \\
\hline 317 & Particle_physics \\
\hline 318 & Materials_science \\
\hline 319 & Computational_biology \\
\hline 320 & Agronomy \\
\hline 321 & Environmental_resource_management \\
\hline 322 & Molecular_biology \\
\hline 323 & Dermatology \\
\hline 324 & Engineering \\
\hline 325 & Surgery \\
\hline 326 & Public_health \\
\hline 327 & Molecular_physics \\
\hline 328 & Bioinformatics \\
\hline 329 & Physics \\
\hline 330 & Cognitive_psychology \\
\hline 331 & Oncology \\
\hline 332 & Pathology \\
\hline 333 & Biochemistry \\
\hline 334 & Social_science \\
\hline 335 & Climatology \\
\hline 336 & Pharmacology \\
\hline 337 & Agriculture \\
\hline 338 & Neuroscience \\
\hline 339 & Fishery \\
\hline 340 & Virology \\
\hline 341 & Microbiology \\
\hline 342 & Communication \\
\hline 343 & Endocrinology \\
\hline 344 & Epigenetics \\
\hline 345 & Marketing \\
\hline 346 & Global_health \\
\hline
\end{tabular}

$-1.530104 \mathrm{e}-01$

$-1.208006 \mathrm{e}-01$

$-1.522810 \mathrm{e}-01$

$4.569468 \mathrm{e}-01$

$-1.413242 \mathrm{e}-01$

$6.541215 \mathrm{e}-02$

$-2.650373 \mathrm{e}-01$

$4.372475 \mathrm{e}-01$

$-1.406031 \mathrm{e}-01$

$-2.919310 \mathrm{e}-02$

$-5.471109 \mathrm{e}-02$

$-1.809289 \mathrm{e}-01$

$-8.711230 \mathrm{e}-02$

$1.109425 \mathrm{e}-01$

$-4.187978 \mathrm{e}-01$

$-2.412514 \mathrm{e}-01$

$-7.128794 \mathrm{e}-01$

$-1.519889 \mathrm{e}-01$

$-3.240712 \mathrm{e}-01$

$-1.401263 \mathrm{e}-01$

$-7.839039 \mathrm{e}-02$

$1.721709 \mathrm{e}-01$

$1.163050 \mathrm{e}-01$

$2.005427 \mathrm{e}-01$

$2.404188 \mathrm{e}-01$

$1.831802 \mathrm{e}-01$

$1.445717 \mathrm{e}-02$

$-1.083858 \mathrm{e}-01$

$-3.712357 \mathrm{e}-02$

$-2.097268 \mathrm{e}-01$

$8.744930 \mathrm{e}-02$

$-6.166676 \mathrm{e}-02$

$1.417851 \mathrm{e}-01$

$1.608861 \mathrm{e}-01$

$1.594539 \mathrm{e}-01$

$-5.391396 \mathrm{e}-02$

$2.401873 \mathrm{e}-01$

$2.880421 \mathrm{e}-01$

$1.435663 \mathrm{e}-01$

$-1.728541 \mathrm{e}-02$

$-5.146881 \mathrm{e}-01$

$-6.886770 \mathrm{e}-02$

$-8.003381 \mathrm{e}-02$

$1.213806 \mathrm{e}-03$

$-2.784589 \mathrm{e}-01$

$1.070506 \mathrm{e}-01$

$-5.254046 \mathrm{e}-03$

$-4.055292 \mathrm{e}-01$

$1.600701 \mathrm{e}-01$

$-7.008866 \mathrm{e}-02$

$2.343603 \mathrm{e}-01$

$-2.252473 \mathrm{e}-01$

$-3.206476 \mathrm{e}-01$

$1.675965 \mathrm{e}-02$

$8.431974 \mathrm{e}-02$

$-1.407800 \mathrm{e}-01$

$-6.209284 \mathrm{e}-02$

$7.226562 \mathrm{e}-02$

$-2.159606 \mathrm{e}-01$

$-1.293220 \mathrm{e}-01$

$-2.126438 \mathrm{e}-01$

8.277387e-02

2.120101e-02

$-6.290769 \mathrm{e}-02$

$-1.303452 \mathrm{e}-01$

$6.594518 \mathrm{e}-02$

$-8.295582 \mathrm{e}-02$

$-2.038808 \mathrm{e}-01$

$6.484000 \mathrm{e}-03$

$-2.102279 \mathrm{e}-01$
0.132771

0.097351

0.097646

0.733914

0.095115

0.256676

0.190140

0.430508

0.206107

0.178686

0.190042

0.124834

0.113108

0.256964

0.287672

0.187498

0.244629

0.169573

0.120671

0.141206

0.090265

0.134234

0.085238

0.180201

0.149166

0.158995

0.117821

0.114745

0.074053

0.231280

0.238827

0.176886

0.183644

0.102834

0.125640

0.107686

0.095808

0.137263

0.114968

0.161338

0.222524

0.081632

0.121947

0.112305

0.074938

0.037830

0.140825

0.141090

0.037936

0.043877

0.149442

0.084063

0.088412

0.053147

0.061431

0.050008

0.046216

0.130312

0.052833

0.068141

0.075827

0.037780

0.081538

0.050882

0.066750

0.071462

0.029428

0.066214

0.096933

0.122478
$2.491446 \mathrm{e}-01$

$2.146544 \mathrm{e}-01$

$1.188768 \mathrm{e}-01$

$5.335389 \mathrm{e}-01$

$1.373320 \mathrm{e}-01$

$7.988452 \mathrm{e}-01$

$1.633507 \mathrm{e}-01$

$3.097973 \mathrm{e}-01$

$4.951242 \mathrm{e}-01$

$8.702223 \mathrm{e}-01$

$7.734325 \mathrm{e}-01$

$1.472420 \mathrm{e}-01$

$4.412005 \mathrm{e}-01$

$6.659285 \mathrm{e}-01$

$1.454482 \mathrm{e}-01$

$1.982064 \mathrm{e}-01$

$3.567925 \mathrm{e}-03$

$3.700929 \mathrm{e}-01$

$7.242106 \mathrm{e}-03$

$3.210271 \mathrm{e}-01$

$3.851550 \mathrm{e}-01$

$1.996289 \mathrm{e}-01$

$1.724250 \mathrm{e}-01$

$2.657617 \mathrm{e}-01$

$1.070207 \mathrm{e}-01$

$2.492776 \mathrm{e}-01$

$9.023411 \mathrm{e}-01$

$3.448750 \mathrm{e}-01$

$6.161517 \mathrm{e}-01$

$3.645117 \mathrm{e}-01$

$7.142457 \mathrm{e}-01$

$7.273718 \mathrm{e}-01$

4.400788e-01

$1.177007 \mathrm{e}-01$

$2.043983 \mathrm{e}-01$

$6.166139 \mathrm{e}-01$

$1.217915 \mathrm{e}-02$

$3.586734 \mathrm{e}-02$

$2.117613 \mathrm{e}-01$

9.146800e-01

$2.072816 \mathrm{e}-02$

$3.988744 \mathrm{e}-01$

$5.116345 \mathrm{e}-01$

$9.913766 \mathrm{e}-01$

$2.026766 \mathrm{e}-04$

$4.659032 \mathrm{e}-03$

$9.702387 \mathrm{e}-01$

$4.050847 \mathrm{e}-03$

$2.452308 \mathrm{e}-05$

$1.101838 \mathrm{e}-01$

$1.168315 \mathrm{e}-01$

$7.374516 \mathrm{e}-03$

$2.872156 \mathrm{e}-04$

$7.524988 \mathrm{e}-01$

$1.698819 \mathrm{e}-01$

4.876691e-03

$1.791067 \mathrm{e}-01$

$5.791993 \mathrm{e}-01$

$4.362709 \mathrm{e}-05$

$5.772042 \mathrm{e}-02$

$5.043597 \mathrm{e}-03$

$2.845838 \mathrm{e}-02$

$7.948548 \mathrm{e}-01$

$2.163372 \mathrm{e}-01$

$5.085319 \mathrm{e}-02$

$3.561116 \mathrm{e}-01$

4.819048e-03

$2.076831 \mathrm{e}-03$

$9.466685 \mathrm{e}-01$

8.608321e-02 


\begin{tabular}{|c|c|c|c|c|c|}
\hline 347 & Agroforestry & $-1.023366 \mathrm{e}-01$ & 0.099613 & $3.042634 \mathrm{e}-01$ & \\
\hline 348 & Chemistry & $-1.706154 \mathrm{e}-01$ & 0.075523 & $2.387944 \mathrm{e}-02$ & $*$ \\
\hline 349 & Mathematics & $9.916813 \mathrm{e}-02$ & 0.172493 & $5.653527 \mathrm{e}-01$ & \\
\hline 350 & Gynecology & $7.714846 \mathrm{e}-03$ & 0.068467 & $9.102853 \mathrm{e}-01$ & \\
\hline 351 & Gerontology & $-1.548258 \mathrm{e}-02$ & 0.051382 & $7.631704 \mathrm{e}-01$ & \\
\hline 352 & Meteorology & $-1.022310 \mathrm{e}-01$ & 0.097902 & $2.963860 \mathrm{e}-01$ & \\
\hline 353 & Veterinary_medicine & $-2.187664 \mathrm{e}-02$ & 0.181025 & $9.038112 \mathrm{e}-01$ & \\
\hline 354 & Government & $5.838730 \mathrm{e}-02$ & 0.087349 & $5.038582 \mathrm{e}-01$ & \\
\hline 355 & Microeconomics & $2.251458 \mathrm{e}-02$ & 0.110058 & $8.379090 \mathrm{e}-01$ & \\
\hline 356 & Sociology & $-3.587626 \mathrm{e}-02$ & 0.102351 & $7.259481 \mathrm{e}-01$ & \\
\hline 357 & Emergency_medicine & $-2.638125 e-02$ & 0.073208 & $7.185775 \mathrm{e}-01$ & \\
\hline 358 & Pediatrics & $-5.868329 \mathrm{e}-02$ & 0.047359 & $2.153019 \mathrm{e}-01$ & \\
\hline 359 & Advertising & $5.823807 \mathrm{e}-02$ & 0.107186 & $5.869006 \mathrm{e}-01$ & \\
\hline 360 & Oceanography & $-2.346723 e-01$ & 0.073053 & $1.317122 \mathrm{e}-03$ & ** \\
\hline 361 & Developmental_psychology & $1.602301 \mathrm{e}-02$ & 0.041542 & $6.997169 \mathrm{e}-01$ & \\
\hline 362 & Internal_medicine & $-6.580158 \mathrm{e}-03$ & 0.045423 & $8.848195 \mathrm{e}-01$ & \\
\hline 363 & Cardiology & $-5.137208 \mathrm{e}-02$ & 0.054119 & $3.425002 \mathrm{e}-01$ & \\
\hline 364 & Environmental_engineering & $-3.475709 \mathrm{e}-01$ & 0.103400 & $7.758290 \mathrm{e}-04$ & $* * *$ \\
\hline 365 & General_surgery & $3.771307 \mathrm{e}-03$ & 0.094628 & $9.682097 \mathrm{e}-01$ & \\
\hline 366 & Environmental_science & $-1.405490 \mathrm{e}-01$ & 0.075904 & $6.407606 \mathrm{e}-02$ & \\
\hline 367 & Geophysics & $-3.276576 \mathrm{e}-01$ & 0.085726 & $1.324129 \mathrm{e}-04$ & $* * *$ \\
\hline 368 & Immunology & $-1.180278 \mathrm{e}-01$ & 0.030886 & $1.327800 \mathrm{e}-04$ & $* * *$ \\
\hline 369 & Clinical_psychology & $-5.710342 \mathrm{e}-03$ & 0.051239 & $9.112639 \mathrm{e}-01$ & \\
\hline 370 & Toxicology & $-1.388905 \mathrm{e}-01$ & 0.118715 & $2.420241 \mathrm{e}-01$ & \\
\hline 371 & Ecology & $-2.204166 \mathrm{e}-01$ & 0.028954 & $2.716346 \mathrm{e}-14$ & $* * *$ \\
\hline 372 & Biology & $3.126888 \mathrm{e}-02$ & 0.063525 & $6.225579 \mathrm{e}-01$ & \\
\hline 373 & Epidemiology & $-6.900839 \mathrm{e}-02$ & 0.049052 & $1.594790 \mathrm{e}-01$ & \\
\hline 374 & Physical_therapy & $-2.429769 \mathrm{e}-02$ & 0.034015 & $4.750274 \mathrm{e}-01$ & \\
\hline 375 & Physiology & $-2.156699 \mathrm{e}-01$ & 0.086821 & $1.299090 \mathrm{e}-02$ & $*$ \\
\hline 376 & Statistics & $-2.583037 \mathrm{e}-01$ & 0.201861 & $2.006857 \mathrm{e}-01$ & \\
\hline 377 & Psychopathology & $-1.340226 \mathrm{e}-01$ & 0.113052 & $2.358237 \mathrm{e}-01$ & \\
\hline 378 & Nursing & $2.394885 \mathrm{e}-02$ & 0.063192 & $7.046985 \mathrm{e}-01$ & \\
\hline 379 & Seismology & $-3.598085 e-01$ & 0.135588 & $7.963458 \mathrm{e}-03$ & ** \\
\hline 380 & History & 8.397447e-02 & 0.193616 & $6.644952 \mathrm{e}-01$ & \\
\hline 381 & Psychiatry & $-8.875945 \mathrm{e}-02$ & 0.038209 & $2.018135 \mathrm{e}-02$ & $*$ \\
\hline 382 & Zoology & $-1.464639 \mathrm{e}-01$ & 0.061093 & $1.651398 \mathrm{e}-02$ & $*$ \\
\hline 383 & Psychology & $-4.680635 e-02$ & 0.066437 & $4.811117 \mathrm{e}-01$ & \\
\hline 384 & Etiology & $1.207033 \mathrm{e}-01$ & 0.165057 & $4.646094 \mathrm{e}-01$ & \\
\hline 385 & Geomorphology & $-1.001681 \mathrm{e}-01$ & 0.067753 & $1.392994 \mathrm{e}-01$ & \\
\hline 386 & Atmospheric_sciences & $-3.118873 e-01$ & 0.065910 & $2.227327 \mathrm{e}-06$ & $* * *$ \\
\hline 387 & Geography & $3.375765 \mathrm{e}-03$ & 0.081065 & $9.667838 \mathrm{e}-01$ & \\
\hline 388 & Demography & $-3.752818 \mathrm{e}-02$ & 0.042629 & $3.786731 \mathrm{e}-01$ & \\
\hline 389 & Geology & $-9.362959 \mathrm{e}-02$ & 0.073806 & $2.045930 \mathrm{e}-01$ & \\
\hline 390 & Medicine & $-1.534967 \mathrm{e}-02$ & 0.059516 & 7.964786e-01 & \\
\hline 391 & Family_medicine & $-6.142130 \mathrm{e}-02$ & 0.049181 & $2.117098 \mathrm{e}-01$ & \\
\hline 392 & Evolutionary_biology & $-1.298161 \mathrm{e}-01$ & 0.053081 & $1.446307 \mathrm{e}-02$ & * \\
\hline 393 & Astrobiology & $-4.705279 e-01$ & 0.079334 & $3.025629 \mathrm{e}-09$ & $* * *$ \\
\hline 394 & Astronomy & $2.001468 \mathrm{e}-02$ & 0.073932 & $7.866079 \mathrm{e}-01$ & \\
\hline 395 & Astrophysics & $-5.991756 \mathrm{e}-02$ & 0.086935 & $4.906845 \mathrm{e}-01$ & \\
\hline 396 & Geochemistry & $-1.471454 \mathrm{e}-01$ & 0.102864 & $1.525830 \mathrm{e}-01$ & \\
\hline 397 & Business & $-1.551947 \mathrm{e}-01$ & 0.099747 & $1.197419 \mathrm{e}-01$ & \\
\hline 398 & Finance & $-1.264973 \mathrm{e}-01$ & 0.125101 & $3.119430 \mathrm{e}-01$ & \\
\hline 399 & Social_psychology & $2.953284 \mathrm{e}-02$ & 0.033976 & $3.847217 \mathrm{e}-01$ & \\
\hline 400 & Political_science & $-1.957042 \mathrm{e}-01$ & 0.099545 & $4.930379 \mathrm{e}-02$ & $*$ \\
\hline 401 & Archaeology & $-5.971080 \mathrm{e}-03$ & 0.085627 & $9.444058 \mathrm{e}-01$ & \\
\hline 402 & Economics & $-2.804262 \mathrm{e}-02$ & 0.074189 & $7.054386 \mathrm{e}-01$ & \\
\hline 403 & Paleontology & $-7.758444 \mathrm{e}-02$ & 0.051442 & $1.315102 \mathrm{e}-01$ & \\
\hline 404 & Criminology & $-4.469132 \mathrm{e}-03$ & 0.158899 & $9.775620 \mathrm{e}-01$ & \\
\hline
\end{tabular}

Table 9: Regression coefficients for predicting sentence-level certainty in news with team size and journal impact factors (RQ4,RQ5). 


\begin{tabular}{|c|c|c|c|c|c|}
\hline & IV & coeff & SE & $\mathrm{p}$ value & \\
\hline 0 & (Intercept) & 4.702461 & $1.967952 \mathrm{e}-02$ & $0.000000 \mathrm{e}+00$ & $* * *$ \\
\hline 1 & finding_len & -0.005205 & $1.466497 \mathrm{e}-04$ & $2.569752 \mathrm{e}-275$ & $* * *$ \\
\hline 2 & flesch_reading_ease & 0.001294 & $5.116505 \mathrm{e}-05$ & $6.128200 \mathrm{e}-141$ & $* * *$ \\
\hline 3 & first_author_rank & 0.000005 & $7.462000 \mathrm{e}-07$ & $1.993841 \mathrm{e}-10$ & $* * *$ \\
\hline 4 & affi_rank & 0.000004 & $8.059055 \mathrm{e}-07$ & $1.423131 \mathrm{e}-06$ & $* * *$ \\
\hline 5 & affi_cateinternational & 0.002711 & $2.850670 \mathrm{e}-03$ & $3.415584 \mathrm{e}-01$ & \\
\hline 6 & affi_cateunknown & -0.059613 & $3.050521 \mathrm{e}-02$ & $5.068047 \mathrm{e}-02$ & \\
\hline 7 & journal_impact_cat>30 & 0.017183 & $6.753183 \mathrm{e}-03$ & $1.094663 \mathrm{e}-02$ & $*$ \\
\hline 8 & journal_impact_cat0 & 0.070931 & $1.583193 \mathrm{e}-02$ & $7.458490 \mathrm{e}-06$ & $* * *$ \\
\hline 9 & journal_impact_cat1 & 0.037022 & $7.467444 \mathrm{e}-03$ & $7.132324 \mathrm{e}-07$ & $* * *$ \\
\hline 10 & journal_impact_cat10 & -0.026817 & $1.123234 \mathrm{e}-02$ & $1.696552 \mathrm{e}-02$ & $*$ \\
\hline 11 & journal_impact_cat11-20 & 0.011012 & $6.338012 \mathrm{e}-03$ & $8.232125 \mathrm{e}-02$ & \\
\hline 12 & journal_impact_cat2 & 0.011039 & $6.455363 \mathrm{e}-03$ & $8.725644 \mathrm{e}-02$ & \\
\hline 13 & journal_impact_cat21-30 & 0.038526 & $9.059239 \mathrm{e}-03$ & $2.112806 \mathrm{e}-05$ & $* * *$ \\
\hline 14 & journal_impact_cat3 & 0.018069 & $6.341746 \mathrm{e}-03$ & $4.383846 \mathrm{e}-03$ & $* *$ \\
\hline 15 & journal_impact_cat4 & 0.004721 & $6.373307 e-03$ & $4.588848 \mathrm{e}-01$ & \\
\hline 16 & journal_impact_cat5 & 0.018826 & $7.066775 \mathrm{e}-03$ & $7.722863 \mathrm{e}-03$ & $* *$ \\
\hline 17 & journal_impact_cat6 & 0.005337 & $7.986480 \mathrm{e}-03$ & $5.039642 \mathrm{e}-01$ & \\
\hline 18 & journal_impact_cat7 & 0.001111 & $8.614447 \mathrm{e}-03$ & $8.973578 \mathrm{e}-01$ & \\
\hline 19 & journal_impact_cat8 & 0.021862 & $9.556599 \mathrm{e}-03$ & $2.215719 \mathrm{e}-02$ & $*$ \\
\hline 20 & num_authors_cat $>30$ & 0.014470 & $1.129322 \mathrm{e}-02$ & $2.000785 \mathrm{e}-01$ & \\
\hline 21 & num_authors_cat1 & -0.148581 & $8.792205 \mathrm{e}-03$ & $4.924378 \mathrm{e}-64$ & $* * *$ \\
\hline 22 & num_authors_cat10 & 0.017420 & $9.410994 \mathrm{e}-03$ & $6.417202 \mathrm{e}-02$ & \\
\hline 23 & num_authors_cat11-20 & 0.028837 & $7.205957 \mathrm{e}-03$ & $6.285478 \mathrm{e}-05$ & $* * *$ \\
\hline 24 & num_authors_cat2 & -0.105479 & $7.601852 \mathrm{e}-03$ & $9.247603 \mathrm{e}-44$ & $* * *$ \\
\hline 25 & num_authors_cat21-30 & 0.038827 & $1.006697 \mathrm{e}-02$ & $1.148614 \mathrm{e}-04$ & $* * *$ \\
\hline 26 & num_authors_cat3 & -0.086735 & $7.493623 \mathrm{e}-03$ & $5.649063 e-31$ & $* * *$ \\
\hline 27 & num_authors_cat4 & -0.056550 & $7.477742 \mathrm{e}-03$ & $3.969260 \mathrm{e}-14$ & $* * *$ \\
\hline 28 & num_authors_cat5 & -0.033781 & $7.570891 \mathrm{e}-03$ & $8.125985 \mathrm{e}-06$ & $* * *$ \\
\hline 29 & num_authors_cat6 & -0.020872 & $7.792431 \mathrm{e}-03$ & $7.394795 \mathrm{e}-03$ & $* *$ \\
\hline 30 & num_authors_cat7 & -0.010136 & $8.040348 \mathrm{e}-03$ & $2.074176 \mathrm{e}-01$ & \\
\hline 31 & num_authors_cat8 & -0.028621 & $8.454277 \mathrm{e}-03$ & $7.109233 \mathrm{e}-04$ & $* * *$ \\
\hline 32 & Law & 0.070084 & $5.388687 \mathrm{e}-02$ & $1.934019 \mathrm{e}-01$ & \\
\hline 33 & Condensed_matter_physics & -0.041109 & $3.862303 \mathrm{e}-02$ & $2.871597 \mathrm{e}-01$ & \\
\hline 34 & Biomedical_engineering & -0.115420 & $7.616218 \mathrm{e}-02$ & $1.296587 \mathrm{e}-01$ & \\
\hline 35 & Atomic_physics & -0.065105 & $6.382480 \mathrm{e}-02$ & $3.077015 \mathrm{e}-01$ & \\
\hline 36 & Development_economics & -0.047205 & $5.816054 \mathrm{e}-02$ & $4.170027 \mathrm{e}-01$ & \\
\hline 37 & Optics & 0.019396 & $4.343975 \mathrm{e}-02$ & $6.552398 \mathrm{e}-01$ & \\
\hline 38 & Public_policy & -0.125913 & $5.683616 \mathrm{e}-02$ & $2.673537 \mathrm{e}-02$ & $*$ \\
\hline 39 & Lung_cancer & -0.006981 & $3.413327 \mathrm{e}-02$ & $8.379544 \mathrm{e}-01$ & \\
\hline 40 & Electronic_engineering & 0.014172 & $6.542282 \mathrm{e}-02$ & $8.285036 \mathrm{e}-01$ & \\
\hline 41 & Artificial_intelligence & -0.247202 & $1.133583 \mathrm{e}-01$ & $2.920580 \mathrm{e}-02$ & $*$ \\
\hline 42 & Machine_learning & -0.055287 & $7.571961 \mathrm{e}-02$ & $4.652959 \mathrm{e}-01$ & \\
\hline 43 & Gender_studies & 0.088098 & $7.363374 \mathrm{e}-02$ & $2.315290 \mathrm{e}-01$ & \\
\hline 44 & Biophysics & -0.095586 & $4.566339 \mathrm{e}-02$ & $3.632583 \mathrm{e}-02$ & $*$ \\
\hline 45 & Analytical_chemistry & 0.188117 & $4.607491 \mathrm{e}-02$ & $4.449884 \mathrm{e}-05$ & $* * *$ \\
\hline 46 & Computer_science & -0.214977 & $5.003685 \mathrm{e}-02$ & $1.736742 \mathrm{e}-05$ & $* * *$ \\
\hline 47 & Radiology & 0.074570 & $3.824252 \mathrm{e}-02$ & $5.118506 \mathrm{e}-02$ & \\
\hline 48 & Urology & 0.152922 & $5.692800 \mathrm{e}-02$ & $7.226562 \mathrm{e}-03$ & $* *$ \\
\hline 49 & Architecture & -0.001120 & $4.665485 \mathrm{e}-02$ & $9.808520 \mathrm{e}-01$ & \\
\hline 50 & Quantum_mechanics & -0.035286 & $6.571753 \mathrm{e}-02$ & $5.913162 \mathrm{e}-01$ & \\
\hline 51 & Particle_physics & 0.087655 & $1.072413 \mathrm{e}-01$ & $4.137200 \mathrm{e}-01$ & \\
\hline 52 & Materials_science & -0.137963 & $3.801369 \mathrm{e}-02$ & $2.842558 \mathrm{e}-04$ & $* * *$ \\
\hline 53 & Computational_biology & -0.208796 & $5.312750 \mathrm{e}-02$ & $8.493616 \mathrm{e}-05$ & $* * *$ \\
\hline 54 & Agronomy & 0.015144 & $5.126088 \mathrm{e}-02$ & $7.676661 \mathrm{e}-01$ & \\
\hline 55 & Environmental_resource_management & -0.314153 & $3.475149 \mathrm{e}-02$ & $1.577128 \mathrm{e}-19$ & $* * *$ \\
\hline 56 & Molecular_biology & 0.187494 & $1.882368 \mathrm{e}-02$ & $2.289362 \mathrm{e}-23$ & $* * *$ \\
\hline 57 & Dermatology & 0.049912 & $5.069291 \mathrm{e}-02$ & $3.248259 \mathrm{e}-01$ & \\
\hline 58 & Engineering & -0.335568 & $6.239112 \mathrm{e}-02$ & $7.519028 \mathrm{e}-08$ & $* * *$ \\
\hline 59 & Surgery & 0.277696 & $1.633176 \mathrm{e}-02$ & $8.381155 \mathrm{e}-65$ & $* * *$ \\
\hline 60 & Public_health & -0.150272 & $2.069503 \mathrm{e}-02$ & $3.846014 \mathrm{e}-13$ & $* * *$ \\
\hline 61 & Molecular_physics & -0.033632 & $7.221638 \mathrm{e}-02$ & $6.414253 \mathrm{e}-01$ & \\
\hline 62 & Bioinformatics & -0.211685 & $3.927797 \mathrm{e}-02$ & $7.075208 \mathrm{e}-08$ & $* * *$ \\
\hline 63 & Physics & -0.215974 & $4.325506 \mathrm{e}-02$ & $5.947209 \mathrm{e}-07$ & $* * *$ \\
\hline 64 & Cognitive_psychology & -0.018843 & $2.709459 \mathrm{e}-02$ & $4.867830 \mathrm{e}-01$ & \\
\hline 65 & Oncology & 0.045282 & $2.996952 \mathrm{e}-02$ & $1.308060 \mathrm{e}-01$ & \\
\hline 66 & Pathology & 0.054385 & $2.291640 \mathrm{e}-02$ & $1.763593 \mathrm{e}-02$ & $*$ \\
\hline
\end{tabular}




\begin{tabular}{|c|c|}
\hline 67 & Biochemistry \\
\hline 68 & Social_science \\
\hline 69 & Climatology \\
\hline 70 & Pharmacology \\
\hline 71 & Agriculture \\
\hline 72 & Neuroscience \\
\hline 73 & Fishery \\
\hline 74 & Virology \\
\hline 75 & Microbiology \\
\hline 76 & Communication \\
\hline 77 & Endocrinology \\
\hline 78 & Epigenetics \\
\hline 79 & Marketing \\
\hline 80 & Global_health \\
\hline 81 & Agroforestry \\
\hline 82 & Chemistry \\
\hline 83 & Mathematics \\
\hline 84 & Gynecology \\
\hline 85 & Gerontology \\
\hline 86 & Meteorology \\
\hline 87 & Veterinary_medicine \\
\hline 88 & Government \\
\hline 89 & Microeconomics \\
\hline 90 & Sociology \\
\hline 91 & Emergency_medicine \\
\hline 92 & Pediatrics \\
\hline 93 & Advertising \\
\hline 94 & Oceanography \\
\hline 95 & Developmental_psychology \\
\hline 96 & Internal_medicine \\
\hline 97 & Cardiology \\
\hline 98 & Environmental_engineering \\
\hline 99 & General_surgery \\
\hline 100 & Environmental_science \\
\hline 101 & Geophysics \\
\hline 102 & Immunology \\
\hline 103 & Clinical_psychology \\
\hline 104 & Toxicology \\
\hline 105 & Ecology \\
\hline 106 & Biology \\
\hline 107 & Epidemiology \\
\hline 108 & Physical_therapy \\
\hline 109 & Physiology \\
\hline 110 & Statistics \\
\hline 111 & Psychopathology \\
\hline 112 & Nursing \\
\hline 113 & Seismology \\
\hline 114 & History \\
\hline 115 & Psychiatry \\
\hline 116 & Zoology \\
\hline 117 & Psychology \\
\hline 118 & Etiology \\
\hline 119 & Geomorphology \\
\hline 120 & Atmospheric_sciences \\
\hline 121 & Geography \\
\hline 122 & Demography \\
\hline 123 & Geology \\
\hline 124 & Medicine \\
\hline 125 & Family_medicine \\
\hline 126 & Evolutionary_biology \\
\hline 127 & Astrobiology \\
\hline 128 & Astronomy \\
\hline 129 & Astrophysics \\
\hline 130 & Geochemistry \\
\hline 131 & Business \\
\hline 132 & Finance \\
\hline 133 & Social_psychology \\
\hline 134 & Political_science \\
\hline 135 & Archaeology \\
\hline 136 & Economics \\
\hline
\end{tabular}

\begin{tabular}{|c|c|c|c|}
\hline 0.150523 & $1.937312 \mathrm{e}-02$ & $7.897200 \mathrm{e}-15$ & $* * *$ \\
\hline-0.077175 & $5.181339 \mathrm{e}-02$ & $1.363622 \mathrm{e}-01$ & \\
\hline-0.105629 & $2.974517 \mathrm{e}-02$ & $3.836280 \mathrm{e}-04$ & $* * *$ \\
\hline-0.066023 & $3.419125 \mathrm{e}-02$ & $5.348590 \mathrm{e}-02$ & \\
\hline-0.124944 & $3.849407 \mathrm{e}-02$ & $1.171319 \mathrm{e}-03$ & $* *$ \\
\hline-0.040433 & $2.052772 \mathrm{e}-02$ & $4.887829 \mathrm{e}-02$ & $*$ \\
\hline-0.018945 & $3.785747 \mathrm{e}-02$ & $6.167764 \mathrm{e}-01$ & \\
\hline 0.044061 & $2.329545 \mathrm{e}-02$ & $5.857037 \mathrm{e}-02$ & \\
\hline 0.062986 & $2.950870 \mathrm{e}-02$ & $3.280375 \mathrm{e}-02$ & * \\
\hline-0.049786 & $3.779225 \mathrm{e}-02$ & $1.877201 \mathrm{e}-01$ & \\
\hline 0.099994 & $1.383730 \mathrm{e}-02$ & $4.973172 \mathrm{e}-13$ & $* * *$ \\
\hline-0.045685 & $3.590429 \mathrm{e}-02$ & $2.032255 \mathrm{e}-01$ & \\
\hline 0.021009 & $4.681376 \mathrm{e}-02$ & $6.535895 \mathrm{e}-01$ & \\
\hline-0.187153 & $5.960169 \mathrm{e}-02$ & $1.689269 \mathrm{e}-03$ & \\
\hline-0.084819 & $4.218669 \mathrm{e}-02$ & $4.437289 \mathrm{e}-02$ & $*$ \\
\hline-0.052805 & $3.598906 \mathrm{e}-02$ & $1.423112 \mathrm{e}-01$ & \\
\hline-0.144235 & $7.229676 \mathrm{e}-02$ & $4.603922 \mathrm{e}-02$ & \\
\hline 0.057809 & $2.921346 \mathrm{e}-02$ & $4.783538 \mathrm{e}-02$ & * \\
\hline-0.017460 & $2.517976 \mathrm{e}-02$ & & \\
\hline-0.151979 & $9613 e-02$ & & \\
\hline & & & $*$ \\
\hline-0.00 & 3.3 & & \\
\hline-0.0 & & & \\
\hline-0.1 & 4.4 & & $* *$ \\
\hline 0.055253 & 3.0 & 6.6 & \\
\hline 0.043768 & 2.15 & $4.188308 \mathrm{e}-02$ & * \\
\hline-0.080519 & 4.98 & 1.062758 & \\
\hline-0.093951 & 7e-02 & 2.543589 & $*$ \\
\hline-0.028208 & $16 \mathrm{e}-02$ & $1.685289 \mathrm{e}-01$ & \\
\hline 0.130101 & 1.96 & $3.449496 \mathrm{e}-11$ & $* * *$ \\
\hline 0.149023 & 2.5 & $5.569803 \mathrm{e}-09$ & $* * *$ \\
\hline-0.195223 & ee-02 & $3 e-05$ & $* * *$ \\
\hline & & & $* *$ \\
\hline-0.410593 & $3 e-02$ & & \\
\hline & & & k* \\
\hline-0.0 & & & $*$ \\
\hline & 2.3 & & \\
\hline 0.0 & 6.1 & & \\
\hline-0.318332 & e-02 & & *** \\
\hline-0.243515 & $e-02$ & $7 e-14$ & $* * *$ \\
\hline-0.088103 & $2.346292 \mathrm{e}-02$ & $77 e-04$ & $* * *$ \\
\hline 0.055652 & $3 e-02$ & $417 \mathrm{e}-04$ & \\
\hline-0.122551 & $4.986490 \mathrm{e}-02$ & $1.398523 \mathrm{e}-02$ & * \\
\hline 0.122982 & $6.827500 \mathrm{e}-02$ & $7.166060 \mathrm{e}-02$ & \\
\hline-0.062601 & $5.205788 \mathrm{e}-02$ & $2.291609 \mathrm{e}-01$ & \\
\hline-0.067656 & $9 \mathrm{e}-02$ & $1.028358 \mathrm{e}-02$ & * \\
\hline-0.239308 & & $1.381345 \mathrm{e}-05$ & \\
\hline-0.196408 & & & \\
\hline & & & $* *$ \\
\hline & 3.6 & & \\
\hline$-0.1-1$ & & & \\
\hline-0.292206 & 6.21 & & \\
\hline-0.054918 & e- 02 & 7.69 & \\
\hline-0.139718 & $3 e-02$ & $4.211423 \mathrm{e}-04$ & $* * *$ \\
\hline-0.345746 & $4.959965 \mathrm{e}-02$ & $3.160046 \mathrm{e}-12$ & \\
\hline 0.017103 & $2.072842 \mathrm{e}-02$ & $4.093193 \mathrm{e}-01$ & \\
\hline-0.296945 & $3.930839 \mathrm{e}-02$ & $4.226897 \mathrm{e}-14$ & \\
\hline-0.137230 & & $3.612458 \mathrm{e}-06$ & $* * *$ \\
\hline-0.117472 & $2.238108 \mathrm{e}-02$ & $1.532959 \mathrm{e}-07$ & $* * *$ \\
\hline-0.242706 & $3.755519 \mathrm{e}-02$ & $1.030608 \mathrm{e}-10$ & $* * *$ \\
\hline-0.417982 & $5.736262 \mathrm{e}-02$ & $3.186050 \mathrm{e}-13$ & $* * *$ \\
\hline-0.206327 & $4.292206 \mathrm{e}-02$ & $1.532825 \mathrm{e}-06$ & $* * *$ \\
\hline-0.142561 & $4.859643 \mathrm{e}-02$ & $3.351218 \mathrm{e}-03$ & \\
\hline & & & \\
\hline-0.350525 & $5.697195 \mathrm{e}-02$ & $7.633308 \mathrm{e}-10$ & \\
\hline-0.057185 & $5.510151 \mathrm{e}-02$ & $2.993578 \mathrm{e}-01$ & \\
\hline 0.016152 & & & \\
\hline-0.262903 & $5.347179 \mathrm{e}-02$ & $8.808093 \mathrm{e}-07$ & \\
\hline & & & \\
\hline-0.227615 & $3.555490 \mathrm{e}-02$ & $1.538332 \mathrm{e}-10$ & \\
\hline
\end{tabular}


Table 10: Regression coefficients for predicting sentence-level certainty in abstract with team size and journal impact factors (RQ4,RQ5). 